\title{
Enantio- and Diastereoselective Synthesis of
}

\section{Chromeno[4,3-b]pyrrole Derivatives bearing Tetra-substituted Chirality Centres through Carbene Catalyzed Cascade}

\section{Reactions}

Tingting Li,${ }^{1 \dagger}$ Jilan Wang,,${ }^{1 \dagger}$ Jun $\mathrm{Xu},{ }^{2,3 \dagger}$ Jiamiao Jin, ${ }^{1}$ Yonggui Robin $\mathrm{Chi}^{1,3}$ and Zhichao Jin ${ }^{1 *}$

${ }^{1}$ Laboratory Breeding Base of Green Pesticide and Agricultural Bioengineering, Key Laboratory of Green Pesticide and Agricultural Bioengineering, Ministry of Education, Guizhou University, Huaxi District, Guiyang 550025, China.

${ }^{2}$ School of Pharmacy, Guizhou University of Traditional Chinese Medicine, Huaxi District, Guiyang 550025, China.

${ }^{3}$ Division of Chemistry \& Biological Chemistry, School of Physical \& Mathematical Sciences, Nanyang Technological University, Singapore 637371, Singapore.

†These authors contributed equally to this work.

E-mail: zcjin@gzu.edu.cn

\section{table of content}

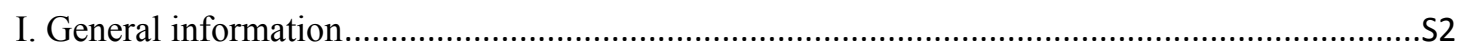

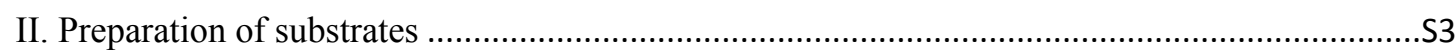

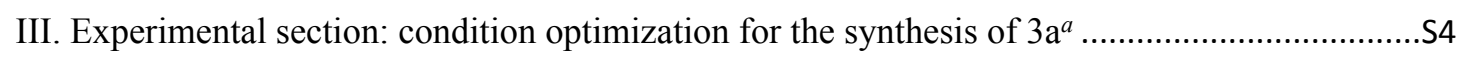

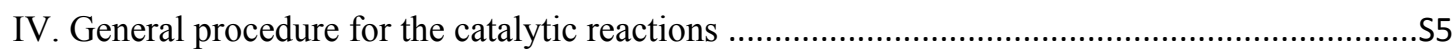

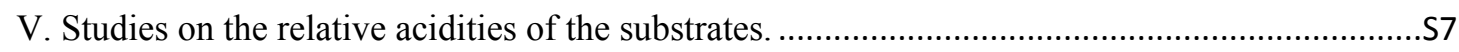

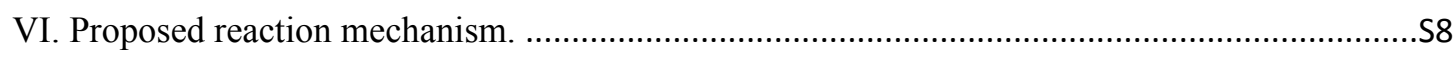

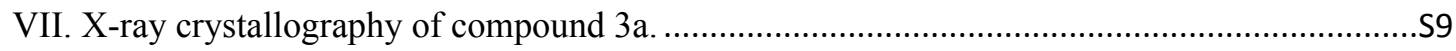

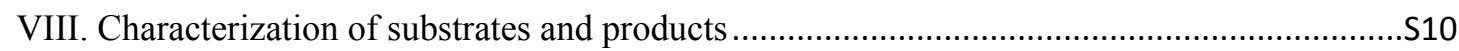

IX. ${ }^{1} \mathrm{H}$ NMR, ${ }^{13} \mathrm{C}$ NMR, ${ }^{19} \mathrm{~F}$ NMR and HPLC spectra......................................................S32 


\section{General information}

Commercially available materials purchased from Energy Chemical and J\&K were used as received. THF was distilled from $\mathrm{Na}$ and used directly. Unless otherwise specified, all reactions were carried out under an atmosphere of Air in $10 \mathrm{~mL}$ Schlenk tube. NMR spectra were measured either on a JEOL-ECX-500 (500 MHz) or on a Bruker ASCEND 400 (400 MHz) spectrometer. The chemical shift values were corrected to $7.26 \mathrm{ppm}\left({ }^{1} \mathrm{H} \mathrm{NMR}\right)$ and $77.16 \mathrm{ppm}\left({ }^{13} \mathrm{C} \mathrm{NMR}\right)$ for $\mathrm{CHCl}_{3}$. $1 \mathrm{H} \mathrm{NMR}$ splitting patterns are designated as singlet (s), double (d), triplet (t), quartet (q), doublet of doublets (dd), multiplets (m), and etc. All first-order splitting patterns were assigned on the base of the appearance of the multiplet. Splitting patterns that could not be easily interpreted are designated as multiplet $(\mathrm{m})$ or broad (br). High resolution mass spectrometer analysis (HRMS) was performed on Thermo Fisher Q Exactive mass spectrometer. HPLC analyses were measured on Waters systems with Empower 3 system controller, Alliance column heater, and 2998 Diode Array Waters 2489 UV/Vis detector. Chiralcel brand chiral columns from Daicel Chemical Industries were used with models IA in 4.6 x $250 \mathrm{~mm}$ size. UPLC analyses were measured on Waters systems with Empower3 system controller, Waters UPLC H-Class, and Waters ACQUITY UPLC PDA detector. Chiralcel brand chiral columns from Daicel Chemical Industries were used with models IA-U, IB-U, IC-U or OD-3 in 3.0 x 100 $\mathrm{mm}$ size. The racemic products used to determine the er values were synthesized using racemic catalyst. Optical rotations were measured on a Insmark IP-digi Polarimeter in a $1 \mathrm{dm}$ cuvette. The concentration (c) is given in $\mathrm{g} / 100 \mathrm{~mL}$. Melting Point (MP): Melting points were measured on a Beijing Tech Instrument X-4 digital display micro melting point apparatus and are uncorrected. Analytical thin-layer chromatography (TLC) was carried out on pre-coated silica gel plate $(0.2 \mathrm{~mm}$ thickness). Visualization was performed using a UV lamp. 


\section{Preparation of substrates}

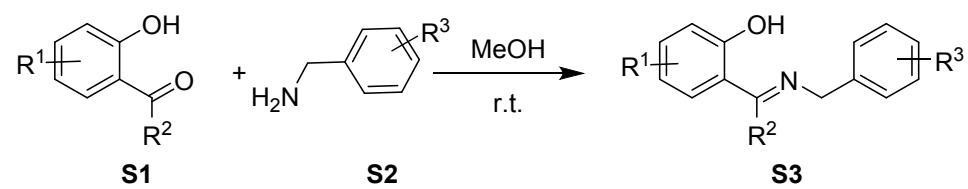

The mixture of S1 (1.0 equiv) and S2 (1.1 equiv) was dissolved with $\mathrm{MeOH}$ stirred overnight at room temperature and a precipitation formed. After stirring, the product was filtered, washed with petroleum ether and vacuum dried to obtained S3. ${ }^{1}$ $\alpha$-Bromoenals were prepared according to reported procedures. ${ }^{2}$

\section{References:}

(1) Qaisi, F. A.; Genjang, N.; Nieger, M.; Repo, T. Inorganica. Chimica. Acta. 2016, 442,81 .

(2) Liu, Y.; Chen, J.; Zhang, Z.; Qin, J.; Zhao, M.; Zhang, W. Org. Biomol. Chem. 2016, 14, 7099. 


\section{Experimental section: condition optimization for the synthesis of $3 \mathrm{a}^{a}$}

\begin{tabular}{|c|c|c|c|c|c|c|c|}
\hline & & $\mathrm{Ar}=4-\mathrm{NO}_{2} \mathrm{C}_{6} \mathrm{H}$ & $\begin{array}{l}\mathrm{NHC}(20 \mathrm{~mol} \%) \\
\text { base }(100 \mathrm{~mol} \%) \\
\begin{array}{c}\mathrm{AMS}(150 \mathrm{mg}) \\
\text { solvent }(2 \mathrm{~mL}) \\
50^{\circ} \mathrm{C}, 12 \mathrm{~h}\end{array}\end{array}$ & & & & \\
\hline entry & $\mathrm{NHC}$ & base & solvent & $\mathrm{T} /{ }^{\circ} \mathrm{C}$ & yield $^{b}$ & e.r. ${ }^{c}$ & $\mathrm{dr}^{d}$ \\
\hline 1 & B & DABCO & THF & 50 & 71 & $96: 4$ & $10: 1$ \\
\hline 2 & A & DABCO(1.0eq.) & THF(1ml) & 50 & 75 & $95: 5$ & - \\
\hline 3 & A & DABCO(1.25eq.) & THF(1ml) & 50 & 83 & $96: 4$ & $11: 1$ \\
\hline 4 & A & DABCO(1.5eq.) & THF(1ml) & 50 & 82 & $96: 4$ & $12: 1$ \\
\hline 5 & A $(20 \%)$ & DABCO & THF(1ml) & 50 & 76 & $96: 4$ & $11: 1$ \\
\hline 6 & A $(10 \%)$ & DABCO & THF(1ml) & 50 & 81 & $97: 3$ & $11: 1$ \\
\hline 7 & $\mathrm{~A}(5 \%)$ & DABCO & THF(1ml) & 50 & 82 & $98: 2$ & $11: 1$ \\
\hline 8 & $\mathrm{~A}(5 \%)$ & DABCO(1.25eq.) & THF(1ml) & 50 & 76 & & \\
\hline 9 & A $(5 \%)$ & DABCO(1.25eq.) & THF(1ml) & 40 & 72 & & \\
\hline 10 & A $(5 \%)$ & DABCO(1.25eq.) & THF(1ml) & 25 & $<5$ & & \\
\hline 11 & $\mathrm{~A}(5 \%)$ & DABCO(1.25eq.) & THF/DCM(1/1) & 50 & 42 & $96: 4$ & $10: 1$ \\
\hline 12 & A $(5 \%)$ & DABCO(1.25eq.) & THF/Tol(1/1) & 50 & 70 & $98: 2$ & $11: 1$ \\
\hline 13 & $\mathrm{~A}(5 \%)$ & DABCO(1.25eq.) & EA/DCM(1/1) & 50 & 82 & $97: 3$ & $7: 1$ \\
\hline 14 & A $(5 \%)$ & DABCO(1.25eq.) & EA/Tol(1/1) & 50 & 84 & $98: 2$ & $10: 1$ \\
\hline 15 & A $(5 \%)$ & DABCO(1.25eq.) & EA/Tol(2/1) & 50 & 83 & $98: 2$ & $14: 1$ \\
\hline 16 & A $(5 \%)$ & DABCO(1.25eq.) & EA/Tol(3/1) & 50 & 84 & $98: 2$ & $11: 1$ \\
\hline 17 & A $(5 \%)$ & DABCO(1.25eq.) & EA/Tol(1/2) & 50 & 79 & $98: 2$ & $16: 1$ \\
\hline$` 18$ & A $(5 \%)$ & DABCO(1.25eq.) & EA/Tol(1/3) & 50 & 77 & $98: 2$ & $12: 1$ \\
\hline 19 & A $(5 \%)$ & DABCO(1.25eq.) & $\mathrm{EA}(1 \mathrm{ml})$ & 50 & 84 & $98: 2$ & $13: 1$ \\
\hline 20 & $\mathrm{~A}(5 \%)$ & DABCO(1.25eq.) & Tol(1ml) & 50 & 77 & $99: 1$ & $>20: 1$ \\
\hline $21^{e}$ & A $(5 \%)$ & DABCO(1.25eq.) & EA/Tol(1/2) & 50 & 82 & $98: 2$ & $19: 1$ \\
\hline $22^{f}$ & A & DABCO & EA & 50 & 75 & $96: 4$ & $8: 1$ \\
\hline \multicolumn{8}{|c|}{$\begin{array}{l}{ }^{a} \text { General conditions (unless otherwise specified): } \mathbf{1 a}(0.10 \mathrm{mmol}), \mathbf{2 a}(0.15 \mathrm{mmol}), \mathrm{NHC}(0.02 \mathrm{mmol}), \text { base }(0.10 \\
\text { mmol), } 4 \AA \mathrm{MS}(150 \mathrm{mg}) \text {, solvent }(2.0 \mathrm{~mL}), 50{ }^{\circ} \mathrm{C}, 12 \mathrm{~h} .{ }^{b} \text { Isolated yield of } \mathbf{3 a} .{ }^{c} \text { Er was determined via HPLC on } \\
\text { chiral stationary phase. }{ }^{d} \text { Dr was determined by }{ }^{1} \mathrm{H} \text { NMR on the crude reaction mixture. }{ }^{e} \mathbf{1 a}(0.10 \mathrm{mmol}), \mathbf{2 a}(0.15\end{array}$} \\
\hline
\end{tabular}




\section{General procedure for the catalytic reactions}

General procedure for the catalytic reactions of 2-Bromoenals $\mathbf{1}$ and substrates $\mathbf{2}$ to synthesize product 3 or $\mathbf{4}$ :

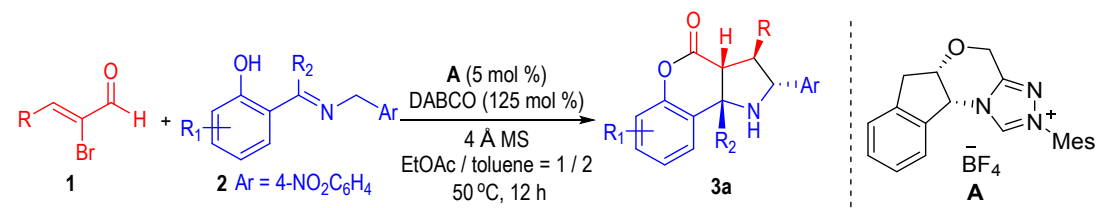

To a $10 \mathrm{~mL}$ flame-dry Schlenk reaction tube equipped with a magnetic stir bar, was added chiral NHC pre-catalyst A (0.005 mmol, $5 \mathrm{~mol} \%, 2.1 \mathrm{mg})$, DABCO (0.125 mmol, $125 \mathrm{~mol} \%, 14 \mathrm{mg}), 4 \AA$ molecular sieves (150 mg), 2-bromoenals 1 (0.1 mmol) and substrates $2(0.15 \mathrm{mmol})$. Freshly distilled anhydrous EtOAc/toluene (1/2, $\mathrm{v} / \mathrm{v}, 1 \mathrm{~mL}$ ) was added via syringe. The reaction mixture was allowed to stir for 12 hours at $50{ }^{\circ} \mathrm{C}$. After completion of the reaction, monitored by TLC plate, The mixture was concentrated under reduced pressure. The resulting crude residue was purified via column chromatography on silica gel (7:1 hexanes/EtOAc) to afford the desired product 3 or $\mathbf{4}$.

Procedure for synthesis of $\mathbf{3 a}$ with gram scale:
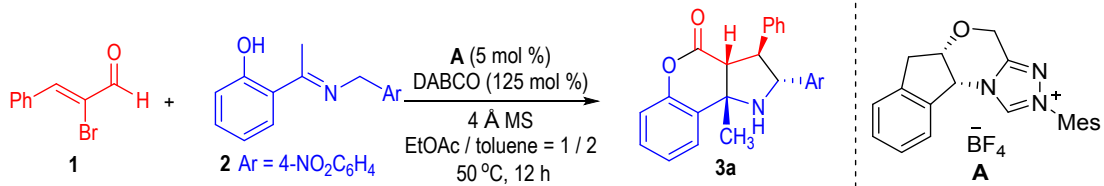

To a $100 \mathrm{~mL}$ flame-dry Schlenk reaction tube equipped with a magnetic stir bar, was added chiral NHC pre-catalyst A (0.25 mmol, $105 \mathrm{mg})$, DABCO (6.25 mmol, $701 \mathrm{mg}), 4 \AA$ molecular sieves (7.5 g), 2-bromoenals $1 \mathrm{a}(5 \mathrm{mmol}, 1.06 \mathrm{~g})$ and aldimines $2 \mathrm{a}$ (7.5 mmol, $2.03 \mathrm{~g})$. Freshly distilled anhydrous EtOAc/toluene (1/2, v/v, $50 \mathrm{~mL}$ ) was added via syringe. The reaction mixture was allowed to stir for 12 hours at $50{ }^{\circ} \mathrm{C}$. After completion of the reaction, monitored by TLC plate, the mixture was concentrated under reduced pressure. The resulting crude residue was purified via column chromatography on silica gel (7:1 hexanes/EtOAc) to afford the desired product 3a (65\% yield, 96\% ee value, 15:1 dr). 
Synthetic transformations and catalytic applications of chiral products $\mathbf{3 a}$ :

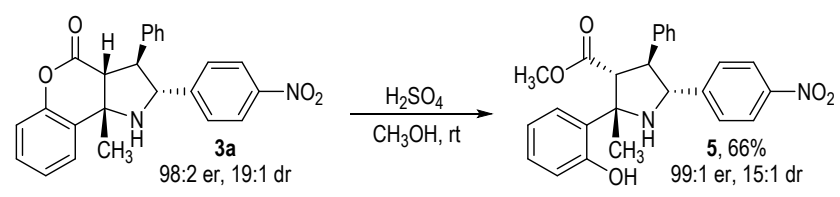

To a $10 \mathrm{~mL}$ round bottom bottle was added compound $\mathbf{3 a}(50 \mathrm{mg})$ and sulfuric acid $(12 \mathrm{mg})$ in methanol $(1 \mathrm{~mL})$ at room temperature, the mixture was stirred for $6 \mathrm{~h}$. Then solvent was removed by a vacuum pump and was purified by chromatography with petroleum ether/EtOAc (2:1) to give 5 (white solid, $66 \%$ yield, 98\% ee, 15:1 dr).

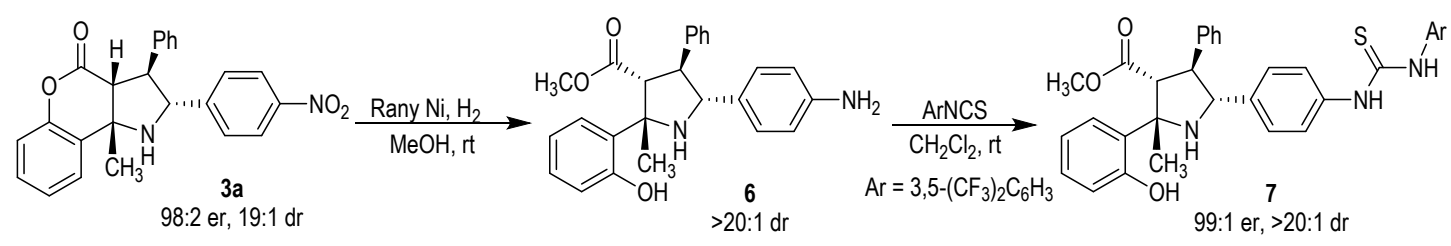

To a $25 \mathrm{~mL}$ flame-dry Schlenk reaction tube equipped with a magnetic stir bar was added compound 3a (280 $\mathrm{mg})$ and excess $\mathrm{Ni}$, the Schlenk tube was sealed with a septum, evacuated and refilled with $\mathrm{H}_{2}$ (3 cycles). Solvent ( $\mathrm{MeOH}, 5.0 \mathrm{~mL}$ ) was then added via syringe. The reaction mixture was allowed to stir for $10 \mathrm{~min}$ at room temperature. After completion of the reaction (monitored by TLC), the mixture was concentrated under reduced pressure. The resulting crude residue was purified via column chromatography on silica gel (5:1 to $2: 1$ petroleum ether /EtOAc) to afford the desired product 6 (white solid, 96\% yield, $>20: 1 \mathrm{dr}$ ).

To a mixture of $\mathrm{CH}_{2} \mathrm{Cl}_{2}(10 \mathrm{~mL})$ and $6(100 \mathrm{mg})$ was added isothiocyanate $(5.0$ $\mathrm{mmol}$ ), and the resulting mixture was stirred at room temperature until TLC indicated the reaction was complete. The reaction mixture was evaporated in vacuum. The pure products 7 were obtained by recrystallization from petroleum ether and ether (white solid, 72\% yield, 99:1 er, >20:1 dr). 


\section{Studies on the relative acidities of the substrates.}

We have examined the H-D exchanging rates of the nucleophilic C(sp3)-H bonds on the substrates bearing different imine groups (as shown in Figure S1).
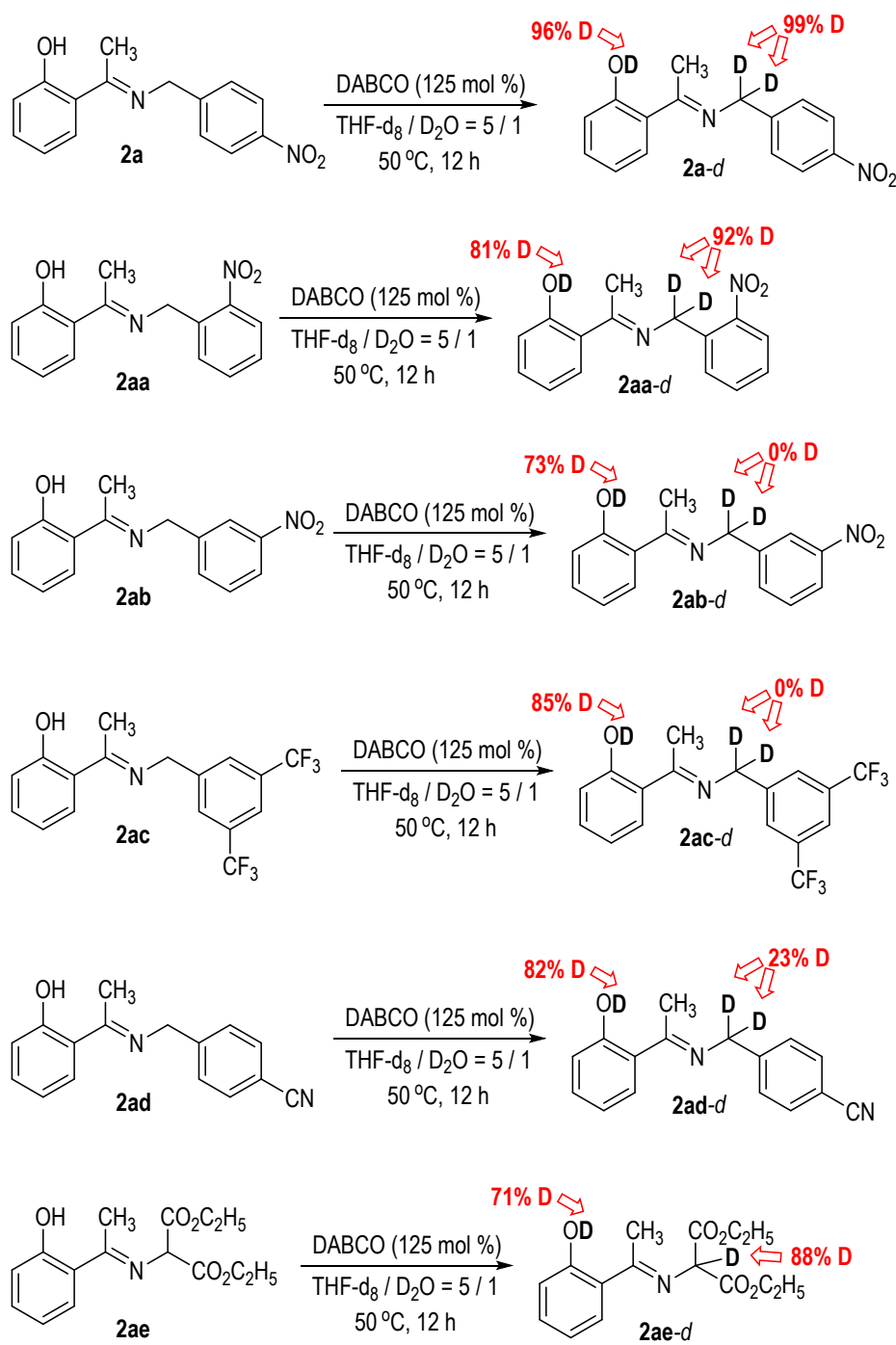

Figure S1. H-D exchanging studies

The phenol $-\mathrm{OH}$ groups of the substrates could be mostly deuterated by D2O under basic conditions in THF-d8. Meanwhile, the nucleophilic $\mathrm{C}(\mathrm{sp} 3)-\mathrm{H}$ bonds of substrates 2a, 2aa could be fully deuterated, the nucleophilic C(sp3)-H bonds of substrates 2ad, 2ae could be partially deuterated, and the nucleophilic C(sp3)-H bonds of substrates $2 \mathbf{a b}, \mathbf{2 a c}$ could not be deuterated. This indicates that the substrates 2a, 2aa could be actvie enough to be deprotonated and react with electrophiles, while the other substrates (2ab, 2ac, 2ad, 2ae) may not be reactive enough. 
Moreover, steric hindrance may also play significant roles in this catalytic cascade reaction. The nucleophilic additions of the substrates $\mathbf{2} \mathbf{a b}$ and $\mathbf{2 a e}$ are much more steric hindered than that of $\mathbf{2 a}$.

Therefore, the cascade reactions using substrates $\mathbf{2 a a}, \mathbf{2} \mathbf{a b}, \mathbf{2} \mathbf{a c}, \mathbf{2} \mathbf{a d}$ and $\mathbf{2 a e}$ could not happen under the current catalytic conditions.

\section{Proposed reaction mechanism.}

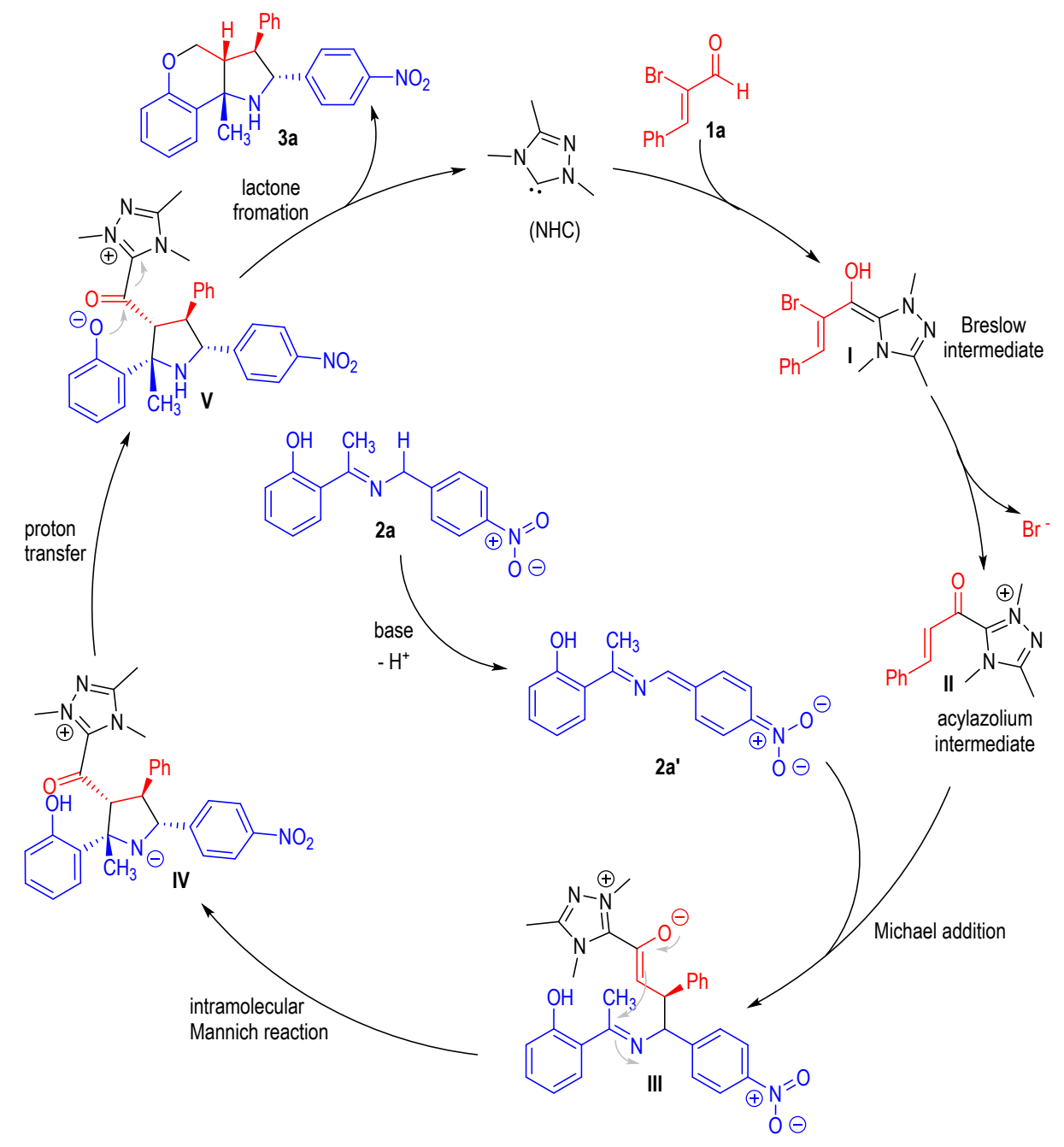

The NHC catalyst can react with the $\beta$-bromo- $\alpha, \beta$-unsaturated enal $\mathbf{1 a}$ and gives the Breslow interemediate I. The $\alpha, \beta$-unsaturated acylazolium intermediate II can be effectively afforded from intermediate $\mathbf{I}$ on loosing of a $\mathrm{Br}^{-}$anion.

The benzylic $\mathrm{C}\left(\mathrm{sp}^{3}\right)-\mathrm{H}$ of the 4-nitrobenyl group in $\mathbf{2 a}$ can be deprotonated by base and generate $\mathbf{2 a}$ ' bearing a reactive nucleophilic benzylic carbon. 2a' Can react with 
the NHC-bound $\alpha, \beta$-unsaturated acylazolium intermediate II through Michael addition and gives intermediate III. A sterically congested tetra-substituted chirality carbon center is formed in excellent diastereoselective fashion during the intramolecular Mannich reaction of the intermediate III and gives intermediate IV bearing a chiral substituted pyrrolidine structure. After a proton transfer process, the final product 3a is readily formed through lactone formation from intermediate $\mathbf{V}$, with the NHC catalyst released for additional catalytic cycles.

\section{X-ray crystallography of compound 3a.}

Good quality crystal of $\mathbf{3 a}$ (yellow block crystal) was obtained by vaporization of a $\mathrm{CH}_{2} \mathrm{Cl}_{2}$ / petroleum ether solution of compound 3a ( 100mg). CCDC 1960143 contains the supplementary crystallographic data for this paper. These data can be obtained free of charge from The Cambridge Crystallographic Data Centre via https://www.ccdc.cam.ac.uk/.
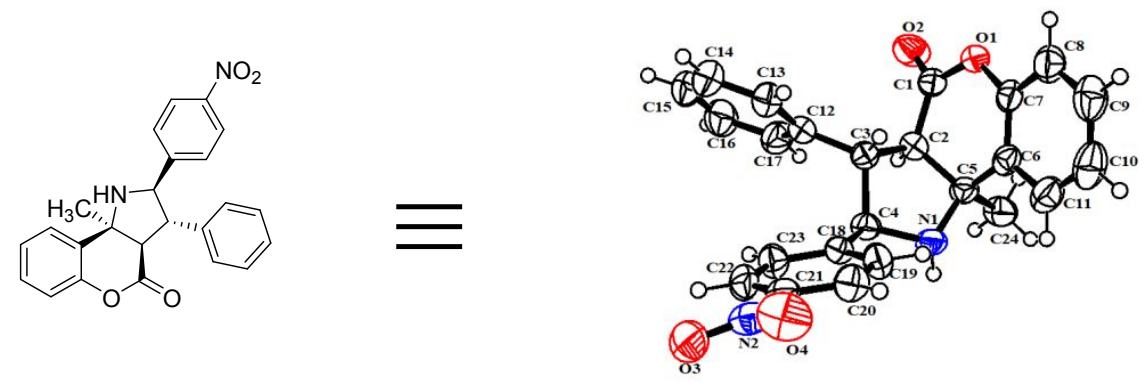
VIII. Characterization of substrates and products

(E)-2-(1-((4-nitrobenzyl)imino)ethyl)phenol (2a):

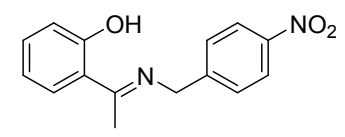

2a

Light yellow solid, 91\% yield, 5.6 g; m.p. $128-130{ }^{\circ} \mathrm{C}$;

1H NMR $\left(400 \mathrm{MHz}, \mathrm{CDCl}_{3}\right) \delta 15.60(\mathrm{~s}, 1 \mathrm{H}), 8.22(\mathrm{~d}, J=8.7 \mathrm{~Hz}$, 2H), $7.62-7.49(\mathrm{~m}, 3 \mathrm{H}), 7.37-7.29(\mathrm{~m}, 1 \mathrm{H}), 6.95(\mathrm{dd}, J=8.3$,

$0.8 \mathrm{~Hz}, 1 \mathrm{H}), 6.90-6.79(\mathrm{~m}, 1 \mathrm{H}), 4.89$ (s, 2H), 2.44 (s, 3H).

${ }^{13} \mathrm{C}$ NMR $\left(101 \mathrm{MHz}, \mathrm{CDCl}_{3}\right) \delta 173.3,162.7,147.2,146.2,132.9,128.3,128.2,124.0$, $119.5,118.4,117.8,53.2,15.1$

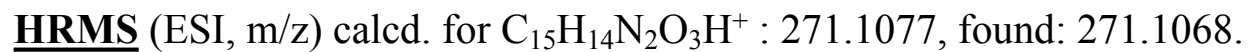

(E)-4-chloro-2-(1-((4-nitrobenzyl)imino)ethyl)phenol (2b):

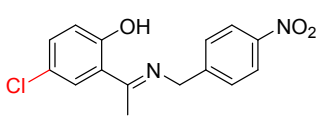

2b, $92 \%$
Light yellow solid, $92 \%$ yield, 1.81 g; m.p. $139-142{ }^{\circ} \mathrm{C}$;

$\underline{1_{\mathbf{H}} \mathbf{N M R}}\left(600 \mathrm{MHz}, \mathrm{CDCl}_{3}\right) \delta 15.54(\mathrm{~s}, 1 \mathrm{H}), 8.23(\mathrm{~d}, J=8.6 \mathrm{~Hz}$, (s, 2H), $2.43(\mathrm{~s}, 3 \mathrm{H})$.

$\underline{{ }^{13} \mathbf{C ~ N M R}}\left(151 \mathrm{MHz}, \mathrm{CDCl}_{3}\right) \delta 172.4,161.3,147.3,145.7,132.7,128.2,127.7,124.1$, $122.4,120.2,119.9,77.3,77.0,76.8,53.3,15.2$.

$\underline{\text { HRMS }}$ (ESI, m/z) calcd. for $\mathrm{C}_{15} \mathrm{H}_{13} \mathrm{~N}_{2} \mathrm{O}_{3} \mathrm{ClH}^{+}$: 305.0687 , found: 305.0697 .

\section{(E)-4-bromo-2-(1-((4-nitrobenzyl)imino)ethyl)phenol (2c):}

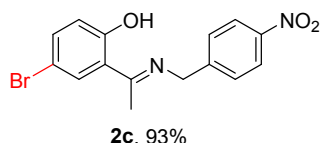

Light yellow solid, $93 \%$ yield, 1.97 g; m.p. $143-149^{\circ} \mathrm{C}$;

$\underline{1 \mathbf{H} \text { NMR }}\left(600 \mathrm{MHz}, \mathrm{CDCl}_{3}\right) \delta 15.54(\mathrm{~s}, 1 \mathrm{H}), 8.23(\mathrm{~d}, J=8.6 \mathrm{~Hz}$,

2H), $7.58-7.49(\mathrm{~m}, 3 \mathrm{H}), 7.27(\mathrm{dd}, J=8.6,2.7 \mathrm{~Hz}, 1 \mathrm{H}), 6.90(\mathrm{~d}, J=8.8 \mathrm{~Hz}, 1 \mathrm{H}), 4.89$

(s, 2H), $2.43(\mathrm{~s}, 3 \mathrm{H})$.

$\underline{{ }^{13} \mathbf{C ~ N M R}}\left(101 \mathrm{MHz}, \mathrm{CDCl}_{3}\right) \delta 172.4,161.8,147.2,145.7,135.4,130.7,128.2,124.0$, $120.8,120.4,109.3,77.4,77.1,76.7,53.2,15.3$.

HRMS (ESI, m/z) calcd. for $\mathrm{C}_{15} \mathrm{H}_{13} \mathrm{~N}_{2} \mathrm{O}_{3} \mathrm{BrH}^{+}$: 349.0182, found: 349.0172. 


\section{(E)-4-fluoro-2-(1-((4-nitrobenzyl)imino)ethyl)phenol (2d):}

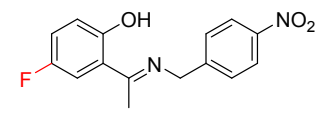

$2 d, 86 \%$

Light yellow solid, $86 \%$ yield, 1.60 g; m.p. $124-138{ }^{\circ} \mathrm{C}$;

$\underline{{ }^{1} \mathbf{H ~ N M R}}\left(600 \mathrm{MHz}, \mathrm{CDCl}_{3}\right) \delta 15.54(\mathrm{~s}, 1 \mathrm{H}), 8.23(\mathrm{~d}, J=8.6 \mathrm{~Hz}$,

2H), $7.58-7.49(\mathrm{~m}, 3 \mathrm{H}), 7.27(\mathrm{dd}, J=8.6,2.7 \mathrm{~Hz}, 1 \mathrm{H}), 6.90(\mathrm{~d}, J=8.8 \mathrm{~Hz}, 1 \mathrm{H}), 4.89$ (s, 2H), $2.43(\mathrm{~s}, 3 \mathrm{H})$.

${ }^{13} \mathrm{C}$ NMR $\left(151 \mathrm{MHz}, \mathrm{CDCl}_{3}\right) \delta 172.3(\mathrm{~d}, J=2.5 \mathrm{~Hz}), 158.6,154.0,147.3,145.9$, 128.3, 124.0, 119.9 (d, $J=23.4 \mathrm{~Hz}), 119.2(\mathrm{~d}, J=7.5 \mathrm{~Hz}), 113.8(\mathrm{~d}, J=24.2 \mathrm{~Hz})$, $53.4,15.3$.

19F NMR $\left(565 \mathrm{MHz}, \mathrm{CDCl}_{3}\right) \delta-125.76$.

$\underline{\text { HRMS }}$ (ESI, m/z) calcd. for $\mathrm{C}_{15} \mathrm{H}_{13} \mathrm{~N}_{2} \mathrm{O}_{3} \mathrm{FH}^{+}$: 289.0983, found: 289.0980.

\section{(E)-4-methyl-2-(1-((4-nitrobenzyl)imino)ethyl)phenol (2e):}

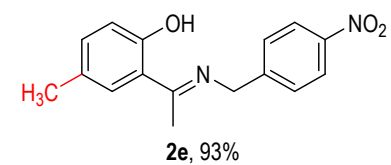

Light yellow solid, $86 \%$ yield, 1.75 g; m.p. $155-159^{\circ} \mathrm{C}$;

${ }^{1} \mathrm{H}$ NMR (400 MHz, $\left.\mathrm{CDCl}_{3}\right) \delta 15.40(\mathrm{~s}, 1 \mathrm{H}), 8.20(\mathrm{~d}, J=8.6$ $\mathrm{Hz}, 2 \mathrm{H}), 7.53$ (d, $J=8.5 \mathrm{~Hz}, 2 \mathrm{H}), 7.37(\mathrm{~s}, 1 \mathrm{H}), 7.14(\mathrm{~d}, J=$

$8.2 \mathrm{~Hz}, 1 \mathrm{H}), 6.86(\mathrm{~d}, J=8.3 \mathrm{~Hz}, 1 \mathrm{H}), 4.86(\mathrm{~s}, 2 \mathrm{H}), 2.41(\mathrm{~s}, 3 \mathrm{H}), 2.31(\mathrm{~s}, 3 \mathrm{H})$.

$\underline{{ }^{13} \mathbf{C ~ N M R}}\left(101 \mathrm{MHz}, \mathrm{CDCl}_{3}\right) \delta 173.2,160.3,147.1,146.4,133.7,128.3,128.2,126.8$, $123.9,119.1,118.0,77.4,77.1,76.8,53.2,20.7,15.1$.

HRMS (ESI, m/z) calcd. for $\mathrm{C}_{16} \mathrm{H}_{16} \mathrm{~N}_{2} \mathrm{O}_{3} \mathrm{H}^{+}: 285.1234$, found: 285.1230 .

(E)-5-methoxy-2-(1-((4-nitrobenzyl)imino)ethyl)phenol (2f):

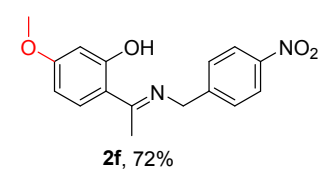

Light yellow solid, 72\% yield, 1.70 g; m.p. 106-113 oC 1. H NMR $\left(600 \mathrm{MHz}, \mathrm{CDCl}_{3}\right) \delta 8.21(\mathrm{~d}, J=8.7 \mathrm{~Hz}, 2 \mathrm{H}), 7.53(\mathrm{~d}, J$ $=8.7 \mathrm{~Hz}, 2 \mathrm{H}), 7.45(\mathrm{~d}, \mathrm{~J}=9.0 \mathrm{~Hz}, 1 \mathrm{H}), 6.43(\mathrm{~d}, \mathrm{~J}=2.5 \mathrm{~Hz}, 1 \mathrm{H})$,

$6.36(\mathrm{dd}, J=9.0,2.6 \mathrm{~Hz}, 1 \mathrm{H}), 4.86(\mathrm{~s}, 2 \mathrm{H}), 3.81(\mathrm{~s}, 3 \mathrm{H}), 2.39(\mathrm{~s}, 3 \mathrm{H})$.

$\underline{{ }^{13} \mathbf{C ~ N M R}}\left(151 \mathrm{MHz}, \mathrm{CDCl}_{3}\right) \delta 172.7,167.9,164.1,147.3,145.7,129.7,128.1,124.0$, $112.6,106.2,102.0,77.3,77.0,76.8,55.4,51.7,14.9$.

HRMS (ESI, m/z) calcd. for $\mathrm{C}_{16} \mathrm{H}_{16} \mathrm{~N}_{2} \mathrm{O}_{4} \mathrm{H}^{+}: 301.1183$, found: 301.1183 . 
(E)-5-chloro-2-(1-((4-nitrobenzyl)imino)ethyl)phenol (2g):

Light yellow solid, 88\% yield, 1.88 g; m.p. 135-137 oC

$\underset{29,88 \%}{1 \mathbf{H ~ N M R}}\left(600 \mathrm{MHz}, \mathrm{CDCl}_{3}\right) \delta 8.23(\mathrm{~d}, J=8.7 \mathrm{~Hz}, 2 \mathrm{H}), 7.53(\mathrm{~d}$, $J=8.6 \mathrm{~Hz}, 2 \mathrm{H}), 7.48(\mathrm{~d}, J=8.6 \mathrm{~Hz}, 1 \mathrm{H}), 6.94(\mathrm{~d}, J=2.1 \mathrm{~Hz}, 1 \mathrm{H}), 6.79$ (dd, $J=8.6$,

$2.1 \mathrm{~Hz}, 1 \mathrm{H}), 4.88(\mathrm{~s}, 2 \mathrm{H}), 2.42(\mathrm{~s}, 3 \mathrm{H})$.

$\underline{{ }^{13} \mathrm{C} \mathrm{NMR}}\left(101 \mathrm{MHz}, \mathrm{CDCl}_{3}\right) \delta 172.9,164.3,147.2,145.6,138.4,129.3,128.2,124.0$, 118.6, 118.0, 117.7, 77.4, 77.1, 76.8, 52.7, 15.2 .

HRMS (ESI, m/z) calcd. for $\mathrm{C}_{15} \mathrm{H}_{13} \mathrm{~N}_{2} \mathrm{O}_{3} \mathrm{H}^{+}:$305.0687, found: 305.0683 .

\section{(E)-5-bromo-2-(1-((4-nitrobenzyl)imino)ethyl)phenol (2h):}

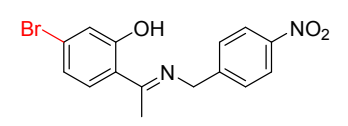

Light yellow solid, $86 \%$ yield, 1.96 g; m.p. $142-144{ }^{\circ} \mathrm{C}$;

2h, $86 \%$

$\underline{{ }^{1} \mathbf{H ~ N M R}}\left(600 \mathrm{MHz}, \mathrm{CDCl}_{3}\right) \delta 8.22(\mathrm{~d}, J=8.7 \mathrm{~Hz}, 2 \mathrm{H}), 7.52(\mathrm{~d}$, $J=8.6 \mathrm{~Hz}, 2 \mathrm{H}), 7.40(\mathrm{~d}, J=8.6 \mathrm{~Hz}, 1 \mathrm{H}), 7.11(\mathrm{~d}, J=2.0 \mathrm{~Hz}, 1 \mathrm{H}), 6.94(\mathrm{dd}, J=8.6$, $2.0 \mathrm{~Hz}, 1 \mathrm{H}), 4.87(\mathrm{~s}, 2 \mathrm{H}), 2.42(\mathrm{~s}, 3 \mathrm{H})$.

${ }^{13} \mathbf{C ~ N M R}\left(101 \mathrm{MHz}, \mathrm{CDCl}_{3}\right) \delta 173.1,164.3,147.2,145.5,129.4,128.2,126.9,124.0$, $121.7,120.8,118.0,77.4,77.1,76.8,52.8,15.2$.

$\underline{\text { HRMS }}$ (ESI, m/z) calcd. for $\mathrm{C}_{15} \mathrm{H}_{13} \mathrm{~N}_{2} \mathrm{O}_{3} \mathrm{BrH}^{+}$: 349.0182, found: 349.0179.

\section{(E)-5-methyl-2-(1-((4-nitrobenzyl)imino)ethyl)phenol (2i):}

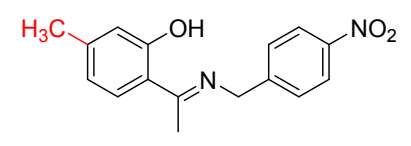

$\mathbf{2 i}, 68 \%$
Light yellow solid, $68 \%$ yield, 1.29 g; m.p. $131-135^{\circ} \mathrm{C}$;

$\underline{1 \text { H NMR }}\left(600 \mathrm{MHz}, \mathrm{CDCl}_{3}\right) \delta 15.64(\mathrm{~s}, 1 \mathrm{H}), 8.22(\mathrm{~d}, J=8.7$

$\mathrm{Hz}, 2 \mathrm{H}), 7.53(\mathrm{~d}, J=8.6 \mathrm{~Hz}, 2 \mathrm{H}), 7.46(\mathrm{~d}, J=8.2 \mathrm{~Hz}, 1 \mathrm{H})$,

$6.76(\mathrm{~s}, 1 \mathrm{H}), 6.66(\mathrm{dd}, J=8.1,0.9 \mathrm{~Hz}, 1 \mathrm{H}), 4.87(\mathrm{~s}, 2 \mathrm{H}), 2.40(\mathrm{~s}, 3 \mathrm{H}), 2.32(\mathrm{~s}, 3 \mathrm{H})$.

${ }^{13} \mathbf{C ~ N M R}\left(151 \mathrm{MHz}, \mathrm{CDCl}_{3}\right) \delta 173.0,162.8,147.2,146.3,143.8,128.2,128.1,124.0$, 119.0, 118.7, 117.1, 77.3, 77.0, 76.8, 53.0, 21.6, 15.0.

HRMS (ESI, m/z) calcd. for $\mathrm{C}_{16} \mathrm{H}_{16} \mathrm{~N}_{2} \mathrm{O}_{3} \mathrm{H}+:$ 285.1234, found: 285.1231. 


\section{(E)-4-nitro-2-(1-((4-nitrobenzyl)imino)ethyl)phenol (2j):}

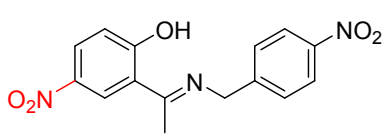

2j, $97 \%$

Light yellow solid, $97 \%$ yield, 1.85 g; m.p. $121-126^{\circ} \mathrm{C}$;

$\underline{{ }^{1} \mathbf{H} \text { NMR }}\left(600 \mathrm{MHz}, \mathrm{CDCl}_{3}\right) \delta 8.59(\mathrm{~d}, J=2.7 \mathrm{~Hz}, 1 \mathrm{H}), 8.28$ $(\mathrm{d}, J=8.7 \mathrm{~Hz}, 2 \mathrm{H}), 8.21(\mathrm{dd}, J=9.3,2.7 \mathrm{~Hz}, 1 \mathrm{H}), 7.57$ (d, $J$

$=8.7 \mathrm{~Hz}, 2 \mathrm{H}), 6.96(\mathrm{~d}, J=9.3 \mathrm{~Hz}, 1 \mathrm{H}), 4.98(\mathrm{~s}, 2 \mathrm{H}), 2.60(\mathrm{~s}, 3 \mathrm{H})$.

${ }^{13} \mathbf{C ~ N M R}\left(151 \mathrm{MHz}, \mathrm{CDCl}_{3}\right) \delta 173.8,171.3,147.6,144.0,137.8,128.6,128.3,125.7$, $124.3,120.5,116.9,77.3,77.1,76.8,52.3,15.2$.

HRMS (ESI, m/z) calcd. for $\mathrm{C}_{15} \mathrm{H}_{13} \mathrm{~N}_{3} \mathrm{O}_{5}^{+}: 315.0850$, found: 315.0839 .

(E)-3-methoxy-2-(1-((4-nitrobenzyl)imino)ethyl)phenol (2k):

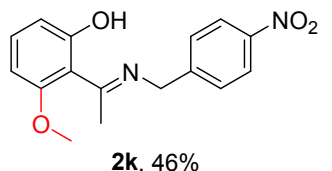

Light yellow solid, 46\% yield, 1.07 g; m.p. 121-126 ${ }^{\circ} \mathrm{C}$;

$\underline{{ }^{1} \mathbf{H ~ N M R}}\left(600 \mathrm{MHz}, \mathrm{CDCl}_{3}\right) \delta 8.20(\mathrm{~d}, J=8.7 \mathrm{~Hz}, 2 \mathrm{H}), 7.54(\mathrm{~d}$, $J=8.7 \mathrm{~Hz}, 2 \mathrm{H}), 7.20(\mathrm{t}, J=8.3 \mathrm{~Hz}, 1 \mathrm{H}), 6.56(\mathrm{dd}, J=8.3,0.8$

$\mathrm{Hz}, 1 \mathrm{H}), 6.34(\mathrm{~d}, J=8.2 \mathrm{~Hz}, 1 \mathrm{H}), 4.82(\mathrm{~s}, 2 \mathrm{H}), 3.84(\mathrm{~s}, 3 \mathrm{H}), 2.50(\mathrm{~s}, 3 \mathrm{H})$.

$\underline{{ }^{13} \mathrm{C} \mathrm{NMR}}\left(151 \mathrm{MHz}, \mathrm{CDCl}_{3}\right) \delta 174.6,164.2,160.1,147.1,146.2,132.4,128.3,124.0$, $111.4,110.9,100.4,77.3,77.1,76.9,55.4,52.1,20.7$.

HRMS (ESI, m/z) calcd. for $\mathrm{C}_{16} \mathrm{H}_{16} \mathrm{~N}_{2} \mathrm{O}_{4} \mathrm{H}^{+}: 301.1183$, found: 301.1179 .

\section{(E)-2-(1-((4-nitrobenzyl)imino)propyl)phenol (2l):}

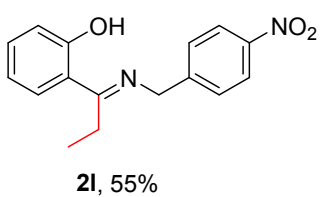

Light yellow solid, 55\% yield, 1.35 g; m.p. $126-128^{\circ} \mathrm{C}$;

$\underline{{ }^{1} \mathbf{H ~ N M R}}\left(600 \mathrm{MHz}, \mathrm{CDCl}_{3}\right) \delta 15.83(\mathrm{~s}, 1 \mathrm{H}), 8.22(\mathrm{~d}, J=8.7 \mathrm{~Hz}$, 2H), $7.59-7.51(\mathrm{~m}, 3 \mathrm{H}), 7.36-7.30(\mathrm{~m}, 1 \mathrm{H}), 6.96(\mathrm{dd}, J=8.3$, $1.0 \mathrm{~Hz}, 1 \mathrm{H}), 6.89-6.82(\mathrm{~m}, 1 \mathrm{H}), 4.92(\mathrm{~s}, 2 \mathrm{H}), 2.88(\mathrm{q}, J=7.8 \mathrm{~Hz}, 2 \mathrm{H}), 1.27(\mathrm{t}, J=$ $7.8 \mathrm{~Hz}, 3 \mathrm{H})$.

$\underline{{ }^{13} \mathrm{C} \mathrm{NMR}}\left(151 \mathrm{MHz}, \mathrm{CDCl}_{3}\right) \delta 178.0,163.5,147.2,146.2,132.9,128.2,124.0,118.7$, $117.9,117.8,77.3,77.1,76.9,52.2,21.4,11.9$.

HRMS (ESI, m/z) calcd. for $\mathrm{C}_{16} \mathrm{H}_{16} \mathrm{~N}_{2} \mathrm{O}_{3} \mathrm{H}^{+}:$285.1234, found: 285.1230 . 


\section{(E)-2-(((4-nitrobenzyl)imino)(phenyl)methyl)phenol (2m):}

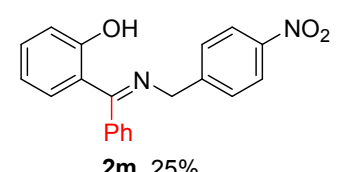

Light yellow solid, $25 \%$ yield, 617 mg; m.p. 152-155 ${ }^{\circ} \mathrm{C}$;

1H NMR $\left(600 \mathrm{MHz}, \mathrm{CDCl}_{3}\right) \delta 15.05(\mathrm{~s}, 1 \mathrm{H}), 8.18(\mathrm{~d}, J=8.7 \mathrm{~Hz}$, 2H), $7.53(\mathrm{dd}, J=5.0,1.7 \mathrm{~Hz}, 3 \mathrm{H}), 7.42(\mathrm{~d}, J=8.6 \mathrm{~Hz}, 2 \mathrm{H})$, $7.35-7.28(\mathrm{~m}, 1 \mathrm{H}), 7.20(\mathrm{dd}, J=6.4,3.0 \mathrm{~Hz}, 2 \mathrm{H}), 7.01(\mathrm{~d}, J=8.2 \mathrm{~Hz}, 1 \mathrm{H}), 6.85(\mathrm{dd}$, $J=8.0,1.5 \mathrm{~Hz}, 1 \mathrm{H}), 6.70(\mathrm{t}, J=7.6 \mathrm{~Hz}, 1 \mathrm{H}), 4.64(\mathrm{~s}, 2 \mathrm{H})$.

$\underline{{ }^{13} \mathrm{C} \mathrm{NMR}}\left(151 \mathrm{MHz}, \mathrm{CDCl}_{3}\right) \delta 176.2,162.6,147.1,146.5,133.7,132.9,131.9,129.4$, 129.2, 129.0, 128.1, 127.1, 123.9, 119.8, 117.9, 117.9, 77.3, 77.1, 76.8, 55.1.

HRMS (ESI, m/z) calcd. for $\mathrm{C}_{20} \mathrm{H}_{16} \mathrm{~N}_{2} \mathrm{O}_{3} \mathrm{H}^{+}: 333.1234$, found: 333.1230 .

\section{(E)-2-(1-((2-nitrobenzyl)imino)ethyl)phenol (2aa)}

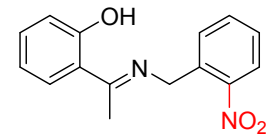

2aa, $37 \%$

Light yellow solid, $37 \%$ yield, $810 \mathrm{mg}$; m.p. $91-92{ }^{\circ} \mathrm{C}$;

1H NMR $\left(600 \mathrm{MHz}, \mathrm{CDCl}_{3}\right) \delta 15.59(\mathrm{~s}, 1 \mathrm{H}), 8.08(\mathrm{~d}, J=8.2 \mathrm{~Hz}$, $1 \mathrm{H}), 7.64(\mathrm{~d}, J=4.1 \mathrm{~Hz}, 2 \mathrm{H}), 7.59(\mathrm{dd}, J=8.0,1.2 \mathrm{~Hz}, 1 \mathrm{H}), 7.47$ $(\mathrm{dp}, J=8.1,4.0 \mathrm{~Hz}, 1 \mathrm{H}), 7.34-7.29(\mathrm{~m}, 1 \mathrm{H}), 6.94(\mathrm{~d}, J=8.3 \mathrm{~Hz}, 1 \mathrm{H}), 6.84(\mathrm{t}, J=$ $7.6 \mathrm{~Hz}, 1 \mathrm{H}), 5.13(\mathrm{~s}, 2 \mathrm{H}), 2.45(\mathrm{~s}, 3 \mathrm{H})$.

${ }^{13} \mathrm{C}$ NMR $\left(151 \mathrm{MHz}, \mathrm{CDCl}_{3}\right) \delta 173.3,162.7,148.2,134.3,133.9,132.7,130.0$, $128.3,128.2,125.2,119.6,118.4,117.8,50.9,15.1$.

HRMS (ESI, m/z) calcd. for $\mathrm{C}_{15} \mathrm{H}_{14} \mathrm{~N}_{2} \mathrm{O}_{3} \mathrm{H}^{+}:$271.1077, found: 271.1077 .

\section{(E)-2-(1-((3-nitrobenzyl)imino)ethyl)phenol (2ab)}

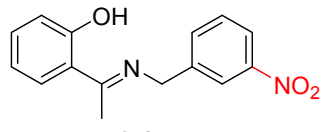

2ab, $80 \%$

light yellow solid, $80 \%$ yield, 2.31 g; m.p. $114-115^{\circ} \mathrm{C}$;

$\underline{1 \mathbf{H} \text { NMR }}\left(600 \mathrm{MHz}, \mathrm{CDCl}_{3}\right) \delta 15.57(\mathrm{~s}, 1 \mathrm{H}), 8.21(\mathrm{~s}, 1 \mathrm{H}), 8.15$ $(\mathrm{dd}, J=8.2,1.4 \mathrm{~Hz}, 1 \mathrm{H}), 7.74(\mathrm{~d}, J=7.6 \mathrm{~Hz}, 1 \mathrm{H}), 7.59$ (dd, $J=$ 8.0, $1.4 \mathrm{~Hz}, 1 \mathrm{H}), 7.55(\mathrm{t}, J=7.9 \mathrm{~Hz}, 1 \mathrm{H}), 7.35-7.29(\mathrm{~m}, 1 \mathrm{H}), 6.94(\mathrm{dd}, J=8.3,0.8$ $\mathrm{Hz}, 1 \mathrm{H}), 6.87-6.82(\mathrm{~m}, 1 \mathrm{H}), 4.87$ (s, 2H), 2.45 (s, 3H).

$\underline{{ }^{13} \mathrm{C} \text { NMR }}\left(151 \mathrm{MHz}, \mathrm{CDCl}_{3}\right) \delta 173.12,162.67,148.45,140.87,133.67,132.80$, $129.83,128.26,122.56,122.37,119.55,118.41,117.81,53.12,15.14$.

HRMS (ESI, m/z) calcd. for $\mathrm{C}_{15} \mathrm{H}_{14} \mathrm{~N}_{2} \mathrm{O}_{3} \mathrm{H}^{+}: 271.1077$, found: 271.1075 . 
(E)-2-(1-((3,5-bis(trifluoromethyl)benzyl)imino)ethyl)phenol (2ac)

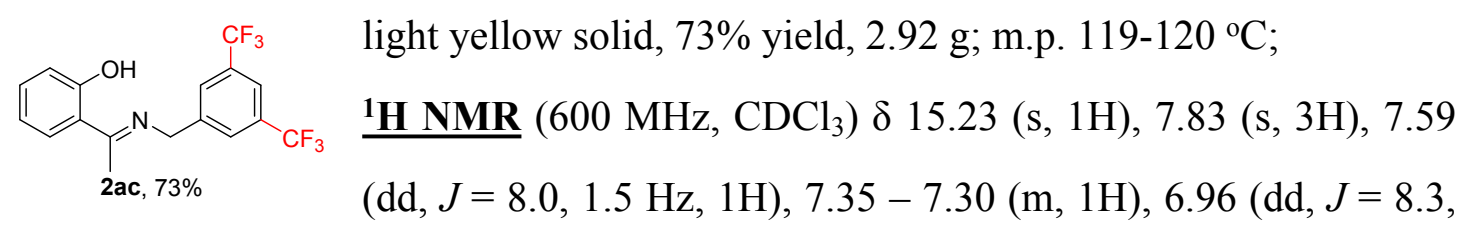
$1.0 \mathrm{~Hz}, 1 \mathrm{H}), 6.88-6.82(\mathrm{~m}, 1 \mathrm{H}), 4.87(\mathrm{~s}, 2 \mathrm{H}), 2.45(\mathrm{~s}, 3 \mathrm{H})$.

$\underline{{ }^{13} \mathrm{C} \mathrm{NMR}}\left(151 \mathrm{MHz}, \mathrm{CDCl}_{3}\right) \delta 173.4,162.5,141.5,132.9,132.1$ (q, $\left.J=33.4 \mathrm{~Hz}\right)$, $128.3,127.8(\mathrm{~d}, J=2.5 \mathrm{~Hz}), 123.3(\mathrm{q}, J=272.6 \mathrm{~Hz}), 121.4(\mathrm{dt}, J=7.5,3.6 \mathrm{~Hz})$, 119.6, 118.4, 118.0, 53.2, 15.3 .

$\underline{{ }^{19} \mathbf{F ~ N M R}}\left(565 \mathrm{MHz}, \mathrm{CDCl}_{3}\right) \delta-62.82$.

$\underline{\text { HRMS }}$ (ESI, m/z) calcd. for $\mathrm{C}_{17} \mathrm{H}_{13} \mathrm{NOF}_{6} \mathrm{H}^{+}$: 362.0974 , found: 362.0970 .

(E)-4-(((1-(2-hydroxyphenyl)ethylidene)amino)methyl)benzonitrile (2ad)

OH light yellow solid, 98\% yield, 2.70 g; m.p. 144-145 ${ }^{\circ} \mathrm{C}$;

2ad, $98 \%$

$\underline{{ }^{1} \mathbf{H ~ N M R}}\left(600 \mathrm{MHz}, \mathrm{CDCl}_{3}\right) \delta 15.67(\mathrm{~s}, 1 \mathrm{H}), 7.65(\mathrm{~d}, J=8.3 \mathrm{~Hz}$, 2H), $7.58(\mathrm{dd}, J=8.0,1.4 \mathrm{~Hz}, 1 \mathrm{H}), 7.48(\mathrm{~d}, J=8.2 \mathrm{~Hz}, 2 \mathrm{H}), 7.36$

- $7.29(\mathrm{~m}, 1 \mathrm{H}), 6.95(\mathrm{dd}, J=8.3,0.8 \mathrm{~Hz}, 1 \mathrm{H}), 6.88-6.80(\mathrm{~m}, 1 \mathrm{H}), 4.84$ (s, 2H), 2.42 $(\mathrm{s}, 3 \mathrm{H})$.

${ }^{13} \mathrm{C}$ NMR $\left(151 \mathrm{MHz}, \mathrm{CDCl}_{3}\right) \delta 173.2,162.8,144.1,132.8,132.6,128.2,128.1$, $119.5,118.7,118.4,117.8,111.2,53.3,15.1$.

HRMS (ESI, m/z) calcd. for $\mathrm{C}_{16} \mathrm{H}_{14} \mathrm{~N}_{2} \mathrm{OH}^{+}$: 251.1179, found: 251.1179. 
(2R,3S,3aR,9bS)-9b-methyl-2-(4-nitrophenyl)-3-phenyl-2,3,3a,9b-tetrahydrochro meno[4,3-b]pyrrol-4(1H)-one (3a)

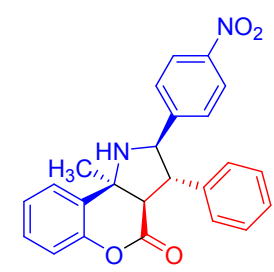

$3 a, 82 \%$ $98: 2 \mathrm{er}, 19: 1 \mathrm{dr}$

White solid, 82\% yield, 33.9 mg; m.p. 194-196 ${ }^{\circ} \mathrm{C}$;

$\lfloor\underline{\alpha}\rfloor^{25} \mathbf{D}=-20.7\left(\mathrm{c}=1.0\right.$ in $\left.\mathrm{CHCl}_{3}\right) ; 19: 1 \mathrm{dr}$ (reaction mixture);

$\underline{1 \mathbf{H} \text { NMR }}\left(400 \mathrm{MHz}, \mathrm{CDCl}_{3}\right) \delta 7.94(\mathrm{~d}, J=8.8 \mathrm{~Hz}, 2 \mathrm{H}), 7.87(\mathrm{dd}, J$ $=5.9,3.5 \mathrm{~Hz}, 1 \mathrm{H}), 7.37-7.27(\mathrm{~m}, 5 \mathrm{H}), 7.14-7.00(\mathrm{~m}, 5 \mathrm{H}), 4.78(\mathrm{~d}$, $J=9.9 \mathrm{~Hz}, 1 \mathrm{H}), 3.52(\mathrm{~d}, J=12.4 \mathrm{~Hz}, 1 \mathrm{H}), 3.21(\mathrm{dd}, J=12.4,9.9 \mathrm{~Hz}$,

1H), $2.77(\mathrm{~s}, 1 \mathrm{H}), 1.67(\mathrm{~s}, 3 \mathrm{H})$.

${ }^{13} \mathrm{C}$ NMR $\left(101 \mathrm{MHz}, \mathrm{CDCl}_{3}\right) \delta 166.8,150.2,148.0,147.1,135.7,129.8,129.2,129.0$, $128.3,128.2,128.0,127.5,125.3,123.3,117.0,77.4,77.3,77.1,76.8,68.9,62.4,58.9$, $58.8,30.0$.

HRMS (ESI) calcd. for $\mathrm{C}_{24} \mathrm{H}_{20} \mathrm{~N}_{2} \mathrm{O}_{4} \mathrm{H}^{+}$: 401.1501, found : 401.1500;

UPLC analysis: 98:2 er (OD-3 column, $25{ }^{\circ} \mathrm{C}$, hexane / iPrOH $=80 / 20,0.5 \mathrm{~mL} /$ $\min , \lambda=254 \mathrm{~nm}), \operatorname{Rt}($ minor $)=2.4 \mathrm{~min}$, Rt (major) $=3.1 \mathrm{~min}$

(2R,3S,3aR,9bS)-3-(2-fluorophenyl)-9b-methyl-2-(4-nitrophenyl)-2,3,3a,9b-tetrah ydrochromeno[4,3-b]pyrrol-4(1H)-one $(3 \mathrm{~b})$

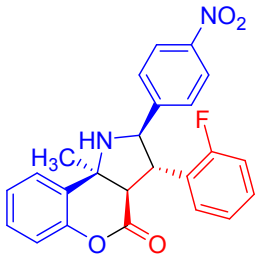

$3 b, 76 \%$

$98: 2 \mathrm{er},>20: 1 \mathrm{dr}$

White solid, $76 \%$ yield, $31.6 \mathrm{mg}$; m.p. $191-192{ }^{\circ} \mathrm{C}$;

$\underline{[\alpha]^{25} \mathbf{D}}=-18.9\left(\mathrm{c}=0.5\right.$ in $\left.\mathrm{CHCl}_{3}\right) ;>20: 1 \mathrm{dr}$ (reaction mixture);

1H NMR $\left(400 \mathrm{MHz}, \mathrm{CDCl}_{3}\right) \delta 8.04-7.90(\mathrm{~m}, 2 \mathrm{H}), 7.90-7.81(\mathrm{~m}$, 1H), $7.40-7.27(\mathrm{~m}, 3 \mathrm{H}), 7.18-6.99(\mathrm{~m}, 6 \mathrm{H}), 4.93(\mathrm{~d}, J=9.8 \mathrm{~Hz}$,

$1 \mathrm{H}), 3.66(\mathrm{~d}, J=12.5 \mathrm{~Hz}, 1 \mathrm{H}), 3.44(\mathrm{dd}, J=12.5,9.9 \mathrm{~Hz}, 1 \mathrm{H}), 2.78$ (s, 1H), $1.68(\mathrm{~s}, 3 \mathrm{H})$.

$\underline{{ }^{13} \mathrm{C} \text { NMR }}\left(101 \mathrm{MHz}, \mathrm{CDCl}_{3}\right) \delta 166.7,161.3(\mathrm{~d}, J=246.8 \mathrm{~Hz}), 150.2,148.0,147.2$, $130.0(\mathrm{dd}, J=6.6,2.0 \mathrm{~Hz}), 129.4,129.3,128.2,127.3,125.3,124.8(\mathrm{~d}, J=3.5 \mathrm{~Hz})$, $123.4,122.9,122.8,117.0,116.3(\mathrm{~d}, J=22.4 \mathrm{~Hz}), 66.7,62.5,57.3,57.2,53.6,30.1$. $\underline{{ }^{19} \mathbf{F} \text { NMR }}\left(377 \mathrm{MHz}, \mathrm{CDCl}_{3}\right) \delta-116.26$.

$\underline{\text { HRMS }}$ (ESI) calcd. for $\mathrm{C}_{24} \mathrm{H}_{19} \mathrm{~N}_{2} \mathrm{O}_{4} \mathrm{FH}^{+}$: 419.1402, found : 419.1402;

UPLC analysis: 98:2 er (IA-U column, $25{ }^{\circ} \mathrm{C}$, hexane / iPrOH $=80 / 20,0.5 \mathrm{~mL} /$ $\min , \lambda=254 \mathrm{~nm}), \mathrm{Rt}($ minor $)=2.5 \mathrm{~min}, \mathrm{Rt}($ major $)=3.2 \mathrm{~min}$ 
(2R,3S,3aR,9bS)-3-(2-chlorophenyl)-9b-methyl-2-(4-nitrophenyl)-2,3,3a,9b-tetrah ydrochromeno[4,3-b]pyrrol-4(1H)-one (3c)

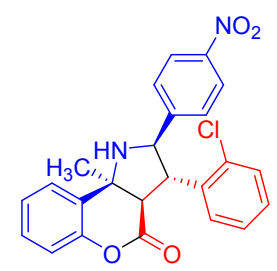

3c, $78 \%$ $99: 1 \mathrm{er},>20: 1 \mathrm{dr}$

White solid, 78\% yield, 33.9 mg; m.p. $230-231{ }^{\circ} \mathrm{C}$;

$\left\lfloor\underline{[\alpha]^{25} \mathbf{D}}=-33.3\left(\mathrm{c}=0.4\right.\right.$ in $\left.\mathrm{CHCl}_{3}\right) ;>20: 1 \mathrm{dr}$ (reaction mixture);

$\underline{{ }^{1} \mathbf{H} \text { NMR }}\left(40 \mathrm{MHz}, \mathrm{CDCl}_{3}\right) \delta 7.97-7.91(\mathrm{~m}, 2 \mathrm{H}), 7.89-7.81(\mathrm{~m}$, 1H), 7.52 (d, $J=7.4 \mathrm{~Hz}, 1 \mathrm{H}), 7.39-7.17(\mathrm{~m}, 5 \mathrm{H}), 7.13$ (d, $J=8.7$ $\mathrm{Hz}, 2 \mathrm{H}), 7.09-7.02(\mathrm{~m}, 1 \mathrm{H}), 4.91(\mathrm{~d}, J=9.9 \mathrm{~Hz}, 1 \mathrm{H}), 3.97$ (dd, $J=$ $12.3,10.1 \mathrm{~Hz}, 1 \mathrm{H}), 3.52(\mathrm{~d}, J=12.5 \mathrm{~Hz}, 1 \mathrm{H}), 2.81(\mathrm{~s}, 1 \mathrm{H}), 1.67(\mathrm{~s}, 3 \mathrm{H})$.

$\underline{{ }^{13} \mathbf{C ~ N M R}}\left(101 \mathrm{MHz}, \mathrm{CDCl}_{3}\right) \delta 166.7,150.0,148.0,147.1,134.8,133.4,130.3,129.4$, $129.3,129.2,128.6,128.1,127.6,127.3,125.3,123.4,116.9,67.6,62.6,58.5,54.0$, 29.9.

$\underline{\text { HRMS }}$ (ESI) calcd. for $\mathrm{C}_{24} \mathrm{H}_{19} \mathrm{~N}_{2} \mathrm{O}_{4} \mathrm{ClH}^{+}: 435.1106$, found : 435.1102;

UPLC analysis: 99:1 er (IA-U column, $25{ }^{\circ} \mathrm{C}$, hexane / $\mathrm{iPrOH}=80 / 20,0.5 \mathrm{~mL} /$ $\min , \lambda=254 \mathrm{~nm}), \mathrm{Rt}($ minor $)=2.6 \mathrm{~min}, \mathrm{Rt}($ major $)=3.8 \mathrm{~min}$.

(2R,3S,3aR,9bS)-3-(2-bromophenyl)-9b-methyl-2-(4-nitrophenyl)-2,3,3a,9b-tetra hydrochromeno[4,3-b]pyrrol-4(1H)-one (3d)

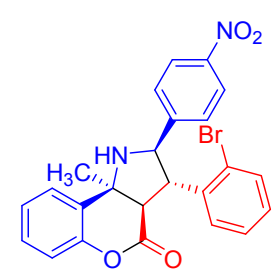

3d, $61 \%$ $99.5: 0.5 \mathrm{er},>20: 1 \mathrm{~d}$

White solid, 61\% yield, $29.2 \mathrm{mg}$; m.p. $215-216{ }^{\circ} \mathrm{C}$;

$\left\lfloor\underline{[\alpha\rfloor^{25} \mathbf{D}}=-30.7\left(\mathrm{c}=1.0\right.\right.$ in $\left.\mathrm{CHCl}_{3}\right) ;>20: 1 \mathrm{dr}($ reaction mixture $) ;$

1H NMR $\left(400 \mathrm{MHz}, \mathrm{CDCl}_{3}\right) \delta 7.98-7.93(\mathrm{~m}, 2 \mathrm{H}), 7.88-7.81(\mathrm{~m}$, 1H), $7.56(\mathrm{~d}, J=7.6 \mathrm{~Hz}, 1 \mathrm{H}), 7.49-7.29(\mathrm{~m}, 4 \mathrm{H}), 7.18-7.04(\mathrm{~m}$, 4H), $4.87(\mathrm{~d}, J=9.8 \mathrm{~Hz}, 1 \mathrm{H}), 4.14-3.90(\mathrm{~m}, 1 \mathrm{H}), 3.46$ (d, $J=12.4$

$\mathrm{Hz}, 1 \mathrm{H}), 2.80(\mathrm{~s}, 1 \mathrm{H}), 1.68(\mathrm{~s}, 3 \mathrm{H})$.

${ }^{13} \mathrm{C}$ NMR $\left(101 \mathrm{MHz}, \mathrm{CDCl}_{3}\right) \delta 166.7,149.8,148.0,147.2,135.1,133.5,129.5,129.4$, $129.4,128.4,128.2,128.1,127.3,125.8,125.3,123.4,116.9,68.1,62.6,59.0,56.1$, 29.8 .

HRMS (ESI) calcd. for $\mathrm{C}_{24} \mathrm{H}_{19} \mathrm{~N}_{2} \mathrm{O}_{4} \mathrm{BrH}^{+}$:479.0601, found : 479.0600;

UPLC analysis: 99.5:0.5 er (IA-U column, $25^{\circ} \mathrm{C}$, hexane / $\mathrm{iPrOH}=80 / 20,0.5 \mathrm{~mL} /$ $\min , \lambda=254 \mathrm{~nm}), \operatorname{Rt}($ minor $)=2.6 \mathrm{~min}$, Rt (major) $=4.0 \mathrm{~min}$ 
(2R,3S,3aR,9bS)-3-(2-methoxyphenyl)-9b-methyl-2-(4-nitrophenyl)-2,3,3a,9b-tetr ahydrochromeno[4,3-b]pyrrol-4(1H)-one (3e)

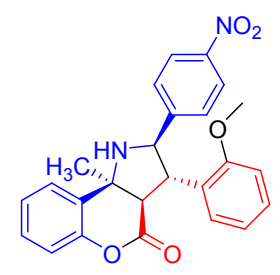

White solid, $72 \%$ yield, $31 \mathrm{mg}$; m.p. $205-206^{\circ} \mathrm{C}$;

$\underline{[\alpha]^{25} \mathbf{D}}=-26.8\left(\mathrm{c}=1.0\right.$ in $\left.\mathrm{CHCl}_{3}\right) ;>20: 1 \mathrm{dr}$ (reaction mixture);

$\underline{\mathbf{1}_{\mathbf{H}} \mathbf{N M R}}\left(400 \mathrm{MHz}, \mathrm{CDCl}_{3}\right) \delta 7.98-7.91(\mathrm{~m}, 2 \mathrm{H}), 7.87-7.81(\mathrm{~m}$, 1H), $7.36-7.23(\mathrm{~m}, 3 \mathrm{H}), 7.20-7.11(\mathrm{~m}, 3 \mathrm{H}), 7.07-7.00(\mathrm{~m}, 1 \mathrm{H})$,

$97: 3 \mathrm{er},>20: 1 \mathrm{dr}$ $6.92(\mathrm{td}, J=7.5,0.9 \mathrm{~Hz}, 1 \mathrm{H}), 6.85(\mathrm{~d}, J=8.2 \mathrm{~Hz}, 1 \mathrm{H}), 5.02(\mathrm{~d}, J=$

$9.3 \mathrm{~Hz}, 1 \mathrm{H}), 3.66(\mathrm{~s}, 3 \mathrm{H}), 3.65-3.58(\mathrm{~m}, 2 \mathrm{H}), 2.73(\mathrm{~s}, 1 \mathrm{H}), 1.64(\mathrm{~s}, 3 \mathrm{H})$.

$\underline{{ }^{13} \mathrm{C} \mathrm{NMR}}\left(101 \mathrm{MHz}, \mathrm{CDCl}_{3}\right) \delta 167.3,157.7,151.3,148.3,146.8,129.6,129.2,129.1$, $128.9,128.2,127.4,125.0,123.8,123.2,121.0,116.8,111.3,65.3,62.5,57.3,55.3$, $53.6,30.0$.

HRMS (ESI) calcd. for $\mathrm{C}_{25} \mathrm{H}_{22} \mathrm{~N}_{2} \mathrm{O}_{5} \mathrm{H}^{+}$: 431.1601, found : 431.1597;

UPLC analysis: 97:3 er (OD-3 column, $25{ }^{\circ} \mathrm{C}$, hexane / $\mathrm{iPrOH}=80 / 20,0.5 \mathrm{~mL} /$ $\min , \lambda=254 \mathrm{~nm}), \operatorname{Rt}($ minor $)=10.0 \mathrm{~min}, \mathrm{Rt}($ major $)=7.4 \mathrm{~min}$

(2R,3S,3aR,9bS)-3-(3-chlorophenyl)-9b-methyl-2-(4-nitrophenyl)-2,3,3a,9b-tetrah ydrochromeno[4,3-b]pyrrol-4(1H)-one (3f)

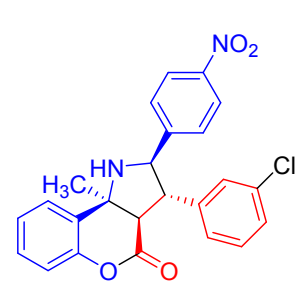

3f, $85 \%$

$98: 2$ er, $7: 1 \mathrm{dr}$

White solid, 85\% yield, $37 \mathrm{mg}$; m.p. $97-98{ }^{\circ} \mathrm{C}$;

$[\boldsymbol{\alpha}]^{25} \mathbf{D}=-13.8\left(\mathrm{c}=1.0\right.$ in $\left.\mathrm{CHCl}_{3}\right) ; 7: 1 \mathrm{dr}($ reaction mixture);

$\underline{{ }^{1} \mathbf{H} \text { NMR }}\left(400 \mathrm{MHz}, \mathrm{CDCl}_{3}\right) \delta 7.99-7.94(\mathrm{~m}, 2 \mathrm{H}), 7.85(\mathrm{dd}, J=$ $6.1,3.3 \mathrm{~Hz}, 1 \mathrm{H}), 7.34(\mathrm{dd}, J=5.9,3.5 \mathrm{~Hz}, 2 \mathrm{H}), 7.29-7.19(\mathrm{~m}$, 2H), $7.17-7.02(\mathrm{~m}, 4 \mathrm{H}), 6.97-6.90(\mathrm{~m}, 1 \mathrm{H}), 4.76(\mathrm{~d}, J=9.8 \mathrm{~Hz}$, 1H), $3.48(\mathrm{~d}, J=12.4 \mathrm{~Hz}, 1 \mathrm{H}), 3.19(\mathrm{dd}, J=12.4,9.9 \mathrm{~Hz}, 1 \mathrm{H}), 2.78(\mathrm{~s}, 1 \mathrm{H}), 1.68(\mathrm{~s}$, $3 \mathrm{H})$.

$\underline{{ }^{13} \mathbf{C ~ N M R}}\left(101 \mathrm{MHz}, \mathrm{CDCl}_{3}\right) \delta 166.5,149.7,147.9,147.3,138.0,134.9,130.3,129.5$, $129.3,128.5,128.2,128.0,127.5,126.5,125.4,123.5,117.0,68.8,62.5,58.9,58.3$, 29.9.

HRMS (ESI) calcd. for $\mathrm{C}_{24} \mathrm{H}_{19} \mathrm{~N}_{2} \mathrm{O}_{4} \mathrm{ClH}^{+}$: 435.1106, found : 435.1104;

UPLC analysis: 98:2 er (IB-U column, $25{ }^{\circ} \mathrm{C}$, hexane / iPrOH $=80 / 20,0.5 \mathrm{~mL} /$ $\min , \lambda=254 \mathrm{~nm}), \operatorname{Rt}($ minor $)=7.9 \mathrm{~min}, \mathrm{Rt}($ major $)=3.0 \mathrm{~min}$. 
(2R,3S,3aR,9bS)-9b-methyl-2-(4-nitrophenyl)-3-(3-(trifluoromethyl)phenyl)-2,3,3 a,9b-tetrahydrochromeno[4,3-b]pyrrol-4(1H)-one $(3 \mathrm{~g})$

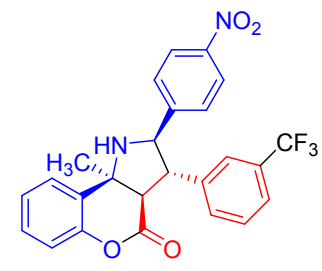

$3 g, 56 \%$ $97: 3$ er, $9: 1 \mathrm{dr}$

White solid, 56\% yield, $26 \mathrm{mg}$; m.p. $84-85{ }^{\circ} \mathrm{C}$;

$\underline{[\alpha\rfloor^{25} \mathbf{D}}=1.8\left(\mathrm{c}=0.3\right.$ in $\left.\mathrm{CHCl}_{3}\right) ; 9: 1 \mathrm{dr}$ (reaction mixture);

$\underline{{ }^{1} \mathbf{H ~ N M R}}\left(400 \mathrm{MHz}, \mathrm{CDCl}_{3}\right) \delta 8.01-7.93(\mathrm{~m}, 2 \mathrm{H}), 7.90-7.83$

(m, 1H), $7.56(\mathrm{~d}, J=7.8 \mathrm{~Hz}, 1 \mathrm{H}), 7.44$ (t, $J=7.8 \mathrm{~Hz}, 1 \mathrm{H}), 7.38-$ $7.32(\mathrm{~m}, 3 \mathrm{H}), 7.26(\mathrm{t}, J=3.8 \mathrm{~Hz}, 1 \mathrm{H}), 7.12-7.03(\mathrm{~m}, 3 \mathrm{H}), 4.77$

$(\mathrm{d}, J=9.8 \mathrm{~Hz}, 1 \mathrm{H}), 3.54(\mathrm{~d}, J=12.5 \mathrm{~Hz}, 1 \mathrm{H}), 3.29$ (dd, $J=12.5,9.9 \mathrm{~Hz}, 1 \mathrm{H}), 2.80(\mathrm{~s}$, $1 \mathrm{H}), 1.70(\mathrm{~s}, 3 \mathrm{H})$.

${ }^{13} \mathrm{C}$ NMR $\left(101 \mathrm{MHz}, \mathrm{CDCl}_{3}\right) \delta 166.4,149.4,147.9,147.3,136.9,131.6,129.6$, $129.5,129.4,128.6,128.1,127.5,125.5,125.19$ (q, $J=3.5 \mathrm{~Hz}), 124.62$ (q, $J=3.6$ Hz), 123.5, 122.8, 117.1, 69.0, 62.5, 58.6, 58.3, 30.0.

19F NMR $\left(377 \mathrm{MHz}, \mathrm{CDCl}_{3}\right) \delta-62.61$.

$\underline{\text { HRMS }}$ (ESI) calcd. for $\mathrm{C}_{25} \mathrm{H}_{19} \mathrm{~N}_{2} \mathrm{O}_{4} \mathrm{~F}_{3} \mathrm{H}^{+}: 469.1370$, found : 469.1366;

UPLC analysis: 97:3 er (IB-U column, $25{ }^{\circ} \mathrm{C}$, hexane / iPrOH $=80 / 20,0.5 \mathrm{~mL} /$ $\min , \lambda=254 \mathrm{~nm})$, Rt (minor) $=8.7 \mathrm{~min}$, Rt (major) $=2.4 \mathrm{~min}$.

(2R,3S,3aR,9bS)-3-(4-fluorophenyl)-9b-methyl-2-(4-nitrophenyl)-2,3,3a,9b-tetrah ydrochromeno[4,3-b]pyrrol-4(1H)-one (3h)

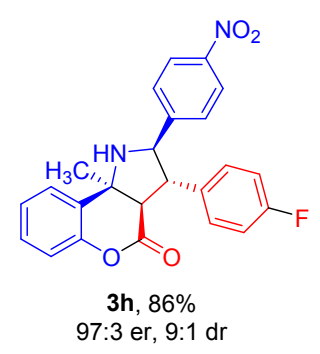

White solid, 86\% yield, $36.0 \mathrm{mg}$; m.p. $173-174{ }^{\circ} \mathrm{C}$;

$\left\lfloor\underline{[\alpha]^{25} \mathbf{D}}=-5.2\left(\mathrm{c}=1.0\right.\right.$ in $\left.\mathrm{CHCl}_{3}\right) ; 9: 1 \mathrm{dr}$ (reaction mixture);

1․ NMR $\left(400 \mathrm{MHz}, \mathrm{CDCl}_{3}\right) \delta 8.03-7.91(\mathrm{~m}, 2 \mathrm{H}), 7.90-7.81$ (m, 1H), $7.38-7.29(\mathrm{~m}, 2 \mathrm{H}), 7.13-6.92(\mathrm{~m}, 7 \mathrm{H}), 4.72(\mathrm{~d}, J=9.9$ Hz, 1H), $3.48(\mathrm{~d}, J=12.5 \mathrm{~Hz}, 1 \mathrm{H}), 3.20(\mathrm{dd}, J=12.5,9.9 \mathrm{~Hz}, 1 \mathrm{H})$,

$2.75(\mathrm{~s}, 1 \mathrm{H}), 1.68(\mathrm{~s}, 3 \mathrm{H})$.

$\left.\underline{{ }^{13} \mathbf{C ~ N M R ~ ( 1 0 1 ~ M H z , ~ C D C l}}{ }_{3}\right) \delta 166.7,162.4(\mathrm{~d}, J=247.1 \mathrm{~Hz}), 149.9,147.9,147.2$, $131.4(\mathrm{~d}, J=3.3 \mathrm{~Hz}), 129.7,129.6(\mathrm{~d}, J=8.1 \mathrm{~Hz}), 129.3,128.2,127.5,125.4,123.4$, 117.0, $116.0(\mathrm{~d}, J=21.4 \mathrm{~Hz}), 69.1,62.3,58.8,58.0,30.0$.

19F NMR (377 MHz, CDCl3) $\delta-113.60$.

$\underline{\text { HRMS }}$ (ESI) calcd. for $\mathrm{C}_{24} \mathrm{H}_{19} \mathrm{~N}_{2} \mathrm{O}_{4} \mathrm{FH}^{+}$: 419.1402, found : 419.1399; 
UPLC analysis: 97:3 er (AD-3 column, $25{ }^{\circ} \mathrm{C}$, hexane / $\mathrm{iPrOH}=80 / 20,0.5 \mathrm{~mL} /$ $\min , \lambda=254 \mathrm{~nm})$, Rt (minor) $=5.3 \mathrm{~min}$, Rt (major) $=6.9 \mathrm{~min}$

(2R,3S,3aR,9bS)-3-(4-chlorophenyl)-9b-methyl-2-(4-nitrophenyl)-2,3,3a,9b-tetrah ydrochromeno[4,3-b]pyrrol-4(1H)-one (3i)

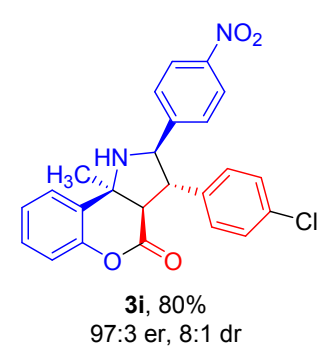

White solid, $80 \%$ yield, 34.9 mg; m.p. $171-172{ }^{\circ} \mathrm{C}$;

$\lfloor\underline{\alpha}\rfloor^{25} \mathbf{D}=-11.2\left(\mathrm{c}=1.0\right.$ in $\left.\mathrm{CHCl}_{3}\right) ; 8: 1 \mathrm{dr}$ (reaction mixture);

$\underline{{ }^{1} \mathbf{H ~ N M R}}\left(400 \mathrm{MHz}, \mathrm{CDCl}_{3}\right) \delta 8.01-7.94(\mathrm{~m}, 2 \mathrm{H}), 7.88-7.83$ (m, 1H), $7.37-7.31(\mathrm{~m}, 2 \mathrm{H}), 7.30-7.26(\mathrm{~m}, 2 \mathrm{H}), 7.11-6.97(\mathrm{~m}$,

$5 \mathrm{H}), 4.72(\mathrm{~d}, J=9.9 \mathrm{~Hz}, 1 \mathrm{H}), 3.48(\mathrm{~d}, J=12.5 \mathrm{~Hz}, 1 \mathrm{H}), 3.19$ (dd, $J=12.5,9.9 \mathrm{~Hz}, 1 \mathrm{H}), 2.75(\mathrm{~s}, 1 \mathrm{H}), 1.68(\mathrm{~s}, 3 \mathrm{H})$.

${ }^{13} \mathrm{C}$ NMR $\left(101 \mathrm{MHz}, \mathrm{CDCl}_{3}\right) \delta 166.6,149.7,147.9,147.2,134.2,134.1,129.6,129.3$, $129.3,128.1,127.5,125.4,123.4,117.0,69.0,62.3,58.7,58.1,30.0$.

HRMS (ESI) calcd. for $\mathrm{C}_{24} \mathrm{H}_{19} \mathrm{~N}_{2} \mathrm{O}_{4} \mathrm{ClH}^{+}$: 435.1106, found : 435.1104;

UPLC analysis: $97: 3 \mathrm{er}\left(\mathrm{IA}-\mathrm{U}\right.$ column, $25^{\circ} \mathrm{C}$, hexane / $\mathrm{iPrOH}=80 / 20,0.5 \mathrm{~mL} / \mathrm{min}$, $\lambda=254 \mathrm{~nm}$ ), Rt (minor) $=2.4 \mathrm{~min}, \operatorname{Rt}($ major $)=3.4 \mathrm{~min}$

(2R,3S,3aR,9bS)-9b-methyl-2,3-bis(4-nitrophenyl)-2,3,3a,9b-tetrahydrochromeno $[4,3-b]$ pyrrol-4(1H)-one $(3 \mathbf{j})$

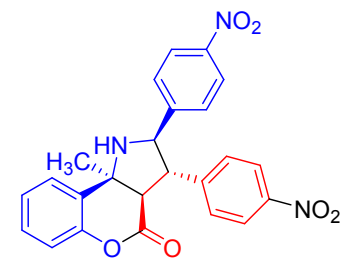

Light yellow solid, 56\% yield, 24.9 mg; m.p. 216-217 ${ }^{\circ} \mathrm{C}$; $\underline{[\alpha]^{25} \mathbf{D}}=-30.9\left(\mathrm{c}=1.0\right.$ in $\left.\mathrm{CHCl}_{3}\right) ; 6: 1 \mathrm{dr}$ (reaction mixture);

1H NMR $\left(400 \mathrm{MHz}, \mathrm{CDCl}_{3}\right) \delta 8.20-8.15(\mathrm{~m}, 2 \mathrm{H}), 8.03-7.96$ $(\mathrm{m}, 2 \mathrm{H}), 7.86(\mathrm{dd}, J=6.0,3.4 \mathrm{~Hz}, 1 \mathrm{H}), 7.42-7.33(\mathrm{~m}, 2 \mathrm{H})$, $93: 7$ er, $6: 1 \mathrm{~d}$ $7.30-7.26(\mathrm{~m}, 3 \mathrm{H}), 7.11-7.05(\mathrm{~m}, 3 \mathrm{H}), 4.78(\mathrm{~d}, J=9.8 \mathrm{~Hz}$,

1H), $3.56(\mathrm{~d}, J=12.5 \mathrm{~Hz}, 1 \mathrm{H}), 3.35$ (dd, $J=12.5,9.9 \mathrm{~Hz}, 1 \mathrm{H}), 2.83(\mathrm{~s}, 1 \mathrm{H}), 1.72(\mathrm{~s}$, $3 \mathrm{H})$.

$\underline{{ }^{13} \mathrm{C} \mathrm{NMR}}\left(101 \mathrm{MHz}, \mathrm{CDCl}_{\underline{3}}\right) \delta 166.2,149.0,147.8,147.7,147.4,143.2,129.5,129.3$, $129.0,128.1,127.5,125.6,124.2,123.6,117.1,69.0,62.5,58.5,58.2,30.0$.

$\underline{\text { HRMS }}$ (ESI) calcd. for $\mathrm{C}_{24} \mathrm{H}_{19} \mathrm{~N}_{3} \mathrm{O}_{6} \mathrm{H}^{+}$: 446.1347, found : 446.1338;

UPLC analysis: 93:7 er (IA-U column, $25^{\circ} \mathrm{C}$, hexane / iPrOH $=80 / 20,0.5 \mathrm{~mL} / \mathrm{min}$, 
$\lambda=254 \mathrm{~nm}$ ), Rt (minor) $=4.5 \mathrm{~min}, \mathrm{Rt}$ (major) $=8.5 \mathrm{~min}$.

(2R,3S,3aR,9bS)-9b-methyl-2-(4-nitrophenyl)-3-(p-tolyl)-2,3,3a,9b-tetrahydrochr omeno[4,3-b]pyrrol-4(1H)-one (3k)

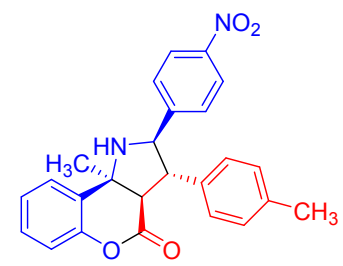

$3 \mathbf{k}, 61 \%$

99.5:0.5 er, $19: 1 \mathrm{dr}$

White solid, 61\% yield, $25.4 \mathrm{mg}$; m.p. $224-226^{\circ} \mathrm{C}$;

$[\alpha]^{25} \mathrm{D}=-6.2\left(\mathrm{c}=1.0\right.$ in $\left.\mathrm{CHCl}_{3}\right) ; 19: 1 \mathrm{dr}$ (reaction mixture);

${ }^{1} \underline{\mathbf{H} \text { NMR }}\left(400 \mathrm{MHz}, \mathrm{CDCl}_{3}\right) \delta 7.97-7.90(\mathrm{~m}, 2 \mathrm{H}), 7.89-7.82$ $(\mathrm{m}, 1 \mathrm{H}), 7.36-7.28(\mathrm{~m}, 2 \mathrm{H}), 7.10(\mathrm{~d}, J=8.6 \mathrm{~Hz}, 4 \mathrm{H}), 7.05-$ $7.00(\mathrm{~m}, 1 \mathrm{H}), 6.97(\mathrm{~d}, J=8.1 \mathrm{~Hz}, 2 \mathrm{H}), 4.76(\mathrm{~d}, J=9.9 \mathrm{~Hz}, 1 \mathrm{H})$,

$3.48(\mathrm{~d}, J=12.4 \mathrm{~Hz}, 1 \mathrm{H}), 3.18(\mathrm{dd}, J=12.4,9.9 \mathrm{~Hz}, 1 \mathrm{H}), 2.76(\mathrm{~s}, 1 \mathrm{H}), 2.31(\mathrm{~s}, 3 \mathrm{H})$, $1.65(\mathrm{~s}, 3 \mathrm{H})$.

$\underline{{ }^{13} \mathrm{C} \mathrm{NMR}}\left(101 \mathrm{MHz}, \mathrm{CDCl}_{3}\right) \delta 166.9,150.4,148.0,147.1,137.9,132.6,129.8,129.7$, $129.2,128.2,127.8,127.5,125.3,123.3,116.9,68.8,62.4,59.1,58.6,30.0,21.2$.

HRMS (ESI) calcd. for $\mathrm{C}_{25} \mathrm{H}_{22} \mathrm{~N}_{2} \mathrm{O}_{4} \mathrm{H}^{+}:$415.1652, found : 415.1653;

UPLC analysis: 99.5:0.5 er (IA-U column, $25^{\circ} \mathrm{C}$, hexane / $\mathrm{PPrOH}=90 / 10,0.5 \mathrm{~mL} /$ $\min , \lambda=254 \mathrm{~nm})$, Rt (minor) $=4.0 \mathrm{~min}$, Rt (major) $=6.0 \mathrm{~min}$.

(2R,3S,3aR,9bS)-3-(4-methoxyphenyl)-9b-methyl-2-(4-nitrophenyl)-2,3,3a,9b-tetr ahydrochromeno $[4,3-b]$ pyrrol-4(1H)-one (3l)

White solid, $85 \%$ yield, $36.5 \mathrm{mg}$; m.p. $150-151^{\circ} \mathrm{C}$;

$\underline{\lfloor\alpha\rfloor^{25} \mathbf{D}}=-24.4\left(\mathrm{c}=1.0\right.$ in $\left.\mathrm{CHCl}_{3}\right) ;>20: 1 \mathrm{dr}$ (reaction mixture);

1H NMR $\left(400 \mathrm{MHz}, \mathrm{CDCl}_{3}\right) \delta 7.97-7.91(\mathrm{~m}, 2 \mathrm{H}), 7.90-7.83(\mathrm{~m}, 1 \mathrm{H}), 7.37-7.29$

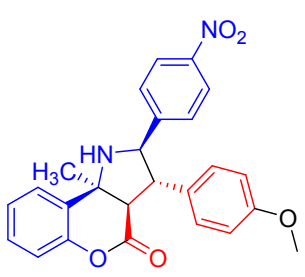

$3 \mathbf{l}, 85 \%$ 99:1 er, $>20: 1 \mathrm{dr}$ $(\mathrm{m}, 2 \mathrm{H}), 7.10(\mathrm{~d}, \mathrm{~J}=8.7 \mathrm{~Hz}, 2 \mathrm{H}), 7.06-6.96(\mathrm{~m}, 3 \mathrm{H}), 6.87-6.78$ $(\mathrm{m}, 2 \mathrm{H}), 4.72(\mathrm{~d}, J=9.9 \mathrm{~Hz}, 1 \mathrm{H}), 3.77(\mathrm{~s}, 3 \mathrm{H}), 3.46(\mathrm{~d}, J=12.4$ $\mathrm{Hz}, 1 \mathrm{H}), 3.16(\mathrm{dd}, J=12.4,9.9 \mathrm{~Hz}, 1 \mathrm{H}), 2.72(\mathrm{~s}, 1 \mathrm{H}), 1.66(\mathrm{~s}$, $3 \mathrm{H})$.

$129.9,129.2,129.0,128.2,127.5,125.3,123.3,116.9,114.4,68.9,62.3,59.0,58.2$, $55.2,30.0$

$\underline{\text { HRMS }}$ (ESI) calcd. for $\mathrm{C}_{25} \mathrm{H}_{22} \mathrm{~N}_{2} \mathrm{O}_{5} \mathrm{H}^{+}: 431.1601$, found : 431.1595; 
UPLC analysis: 99:1 er (IB-U column, $25{ }^{\circ} \mathrm{C}$, hexane / iPrOH $=80 / 20,0.5 \mathrm{~mL} /$ $\min , \lambda=254 \mathrm{~nm})$, Rt (minor) $=11.0 \mathrm{~min}$, Rt (major) $=4.5 \mathrm{~min}$.

(2R,3S,3aR,9bS)-3-(4-(dimethylamino)phenyl)-9b-methyl-2-(4-nitrophenyl)-2,3,3 a,9b-tetrahydrochromeno[4,3-b]pyrrol-4(1H)-one (3m)

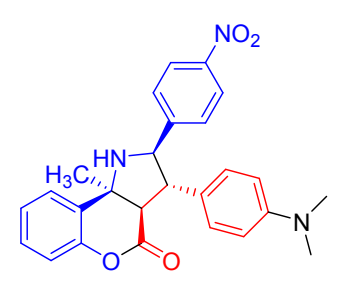

$3 \mathrm{~m}, 89 \%$
$99: 1 \mathrm{er},>20: 1 \mathrm{dr}$
White solid, $89 \%$ yield, $39.3 \mathrm{mg}$; m.p. $195-196^{\circ} \mathrm{C}$;

$\underline{[\alpha\rfloor^{25} \mathbf{D}}=-48.2\left(\mathrm{c}=1.0\right.$ in $\left.\mathrm{CHCl}_{3}\right) ;>20: 1 \mathrm{dr}($ reaction mixture $) ;$

$\underline{{ }^{1} \mathbf{H ~ N M R}}\left(400 \mathrm{MHz}, \mathrm{CDCl}_{3}\right) \delta 7.97-7.92(\mathrm{~m}, 2 \mathrm{H}), 7.90-7.83$

$(\mathrm{m}, 1 \mathrm{H}), 7.36-7.28(\mathrm{~m}, 2 \mathrm{H}), 7.16-7.09(\mathrm{~m}, 2 \mathrm{H}), 7.07-7.00$ (m, 1H), $6.97-6.89(\mathrm{~m}, 2 \mathrm{H}), 6.67-6.56(\mathrm{~m}, 2 \mathrm{H}), 4.72(\mathrm{~d}, J=$

$9.8 \mathrm{~Hz}, 1 \mathrm{H}), 3.44(\mathrm{~d}, J=12.4 \mathrm{~Hz}, 1 \mathrm{H}), 3.12(\mathrm{dd}, J=12.4,9.9 \mathrm{~Hz}, 1 \mathrm{H}), 2.93$ (s, 6H), $1.65(\mathrm{~s}, 3 \mathrm{H})$.

${ }^{13} \mathbf{C}$ NMR $\left(101 \mathrm{MHz}, \mathrm{CDCl}_{3}\right) \delta 167.1,150.7,150.1,148.0,147.0,129.9,129.0,128.6$, $128.2,127.5,125.1,123.2,122.6,116.9,112.6,68.8,62.2,59.1,58.3,40.4,30.0$.

$\underline{\text { HRMS }}$ (ESI) calcd. for $\mathrm{C}_{26} \mathrm{H}_{25} \mathrm{~N}_{3} \mathrm{O}_{4} \mathrm{H}^{+}$: 444.1918, found : 444.1911;

UPLC analysis: 99:1 er (IA-U column, $25{ }^{\circ} \mathrm{C}$, hexane / $\mathrm{iPrOH}=90 / 10,0.5 \mathrm{~mL} /$ $\min , \lambda=254 \mathrm{~nm}), \operatorname{Rt}($ minor $)=6.9 \mathrm{~min}, \mathrm{Rt}($ major $)=10.0 \mathrm{~min}$

(2R,3R,3aR,9bS)-3-(furan-2-yl)-9b-methyl-2-(4-nitrophenyl)-2,3,3a,9b-tetrahydro chromeno[4,3-b]pyrrol-4(1H)-one (3n)

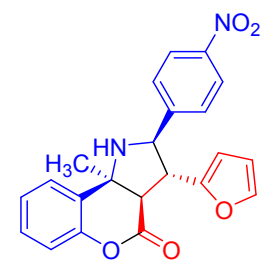

$3 n, 73 \%$ 98:2 er, $6: 1 \mathrm{dr}$

$\mathrm{O}_{2}$ White solid, 73\% yield, $28.6 \mathrm{mg}$; m.p. $140-142{ }^{\circ} \mathrm{C}$;

$\underline{[\alpha]^{25} \mathbf{D}}=-25.6\left(\mathrm{c}=1.0\right.$ in $\left.\mathrm{CHCl}_{3}\right) ; 6: 1 \mathrm{dr}$ (reaction mixture);

$\underline{1 \mathbf{H} \text { NMR }}\left(400 \mathrm{MHz}, \mathrm{CDCl}_{3}\right) \delta 8.05-7.95(\mathrm{~m}, 2 \mathrm{H}), 7.87-7.77(\mathrm{~m}$, 1H), 7.45 (d, $J=1.2 \mathrm{~Hz}, 1 \mathrm{H}), 7.33-7.28$ (m, 2H), 7.19 (d, $J=8.6$ $\mathrm{Hz}, 2 \mathrm{H}), 7.08-6.98(\mathrm{~m}, 1 \mathrm{H}), 6.31(\mathrm{dd}, J=3.2,1.9 \mathrm{~Hz}, 1 \mathrm{H}), 6.04(\mathrm{~d}$, $J=2.9 \mathrm{~Hz}, 1 \mathrm{H}), 4.95(\mathrm{~d}, J=9.7 \mathrm{~Hz}, 1 \mathrm{H}), 3.54(\mathrm{dd}, J=12.1,6.4 \mathrm{~Hz}, 1 \mathrm{H}), 3.33(\mathrm{dd}, J$ =12.0, $9.7 \mathrm{~Hz}, 1 \mathrm{H}), 2.70(\mathrm{~s}, 1 \mathrm{H}), 1.66(\mathrm{~s}, 4 \mathrm{H})$.

${ }^{13} \mathrm{C}$ NMR $\left(101 \mathrm{MHz}, \mathrm{CDCl}_{3}\right) \delta 165.5,149.1,148.1,147.0,146.1,141.9,128.3,128.0$, 127.1, 126.3, 124.3, 122.4, 115.9, 109.6, 108.2, 64.3, 61.4, 56.1, 50.9, 28.6.

$\underline{\text { HRMS }}$ (ESI) calcd. for $\mathrm{C}_{22} \mathrm{H}_{18} \mathrm{~N}_{2} \mathrm{O}_{5} \mathrm{H}^{+}:$391.1288, found : 391.1287; 
UPLC analysis: 98:2 er (IB-U column, $25{ }^{\circ} \mathrm{C}$, hexane / iPrOH $=80 / 20,0.5 \mathrm{~mL} /$ $\min , \lambda=254 \mathrm{~nm}$ ), Rt (minor) $=4.6 \mathrm{~min}$, Rt (major) $=3.1 \mathrm{~min}$.

(2R,3R,3aR,9bS)-9b-methyl-2-(4-nitrophenyl)-3-(thiophen-2-yl)-2,3,3a,9b-tetrahy drochromeno[4,3-b]pyrrol-4(1H)-one (3o)

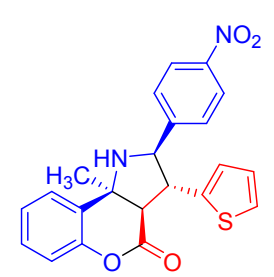

$30,60 \%$

99:1 er, $7: 1 \mathrm{dr}$

White solid, 60\% yield, $24.5 \mathrm{mg}$; m.p. $169-171^{\circ} \mathrm{C}$;

$\underline{\lfloor\alpha\rfloor^{25} \mathbf{D}}=-5.3\left(\mathrm{c}=1.0\right.$ in $\left.\mathrm{CHCl}_{3}\right) ; 7: 1 \mathrm{dr}$ (reaction mixture);

$\underline{{ }^{1} \mathbf{H ~ N M R}}\left(400 \mathrm{MHz}, \mathrm{CDCl}_{3}\right) \delta 7.98(\mathrm{~d}, J=8.8 \mathrm{~Hz}, 2 \mathrm{H}), 7.86-7.80$ (m, 1H), $7.36-7.29(\mathrm{~m}, 2 \mathrm{H}), 7.27$ (d, $J=5.3 \mathrm{~Hz}, 1 \mathrm{H}), 7.20(\mathrm{~d}, J=$ $8.7 \mathrm{~Hz}, 2 \mathrm{H}), 7.09-7.02(\mathrm{~m}, 1 \mathrm{H}), 6.90(\mathrm{dd}, J=5.1,3.5 \mathrm{~Hz}, 1 \mathrm{H}), 6.71$

$(\mathrm{d}, J=2.9 \mathrm{~Hz}, 1 \mathrm{H}), 4.79(\mathrm{~d}, J=9.6 \mathrm{~Hz}, 1 \mathrm{H}), 3.56(\mathrm{dd}, J=12.1,9.7 \mathrm{~Hz}, 1 \mathrm{H}), 3.43(\mathrm{~d}$, $J=12.1 \mathrm{~Hz}, 1 \mathrm{H}), 2.75(\mathrm{~s}, 1 \mathrm{H}), 1.67(\mathrm{~s}, 3 \mathrm{H})$.

${ }^{13} \mathrm{C}$ NMR $\left(101 \mathrm{MHz}, \mathrm{CDCl}_{3}\right) \delta 166.4,149.7,147.9,147.2,139.3,129.4,129.3,128.1$, $127.6,127.2,126.8,125.3,125.3,123.4,117.0,69.7,62.5,60.5,54.0,29.8$.

HRMS (ESI) calcd. for $\mathrm{C}_{22} \mathrm{H}_{18} \mathrm{~N}_{2} \mathrm{O}_{4} \mathrm{SH}^{+}: 407.1060$, found : 407.1055;

UPLC analysis: 99:1 er (OD-3 column, $25^{\circ} \mathrm{C}$, hexane / $\mathrm{iPrOH}=80 / 20,0.5 \mathrm{~mL} /$ $\min , \lambda=254 \mathrm{~nm})$, Rt (minor) $=12.3 \mathrm{~min}$, Rt (major) $=7.9 \mathrm{~min}$

(2R,3R,3aR,9bS)-3,9b-dimethyl-2-(4-nitrophenyl)-2,3,3a,9b-tetrahydrochromeno[ 4,3-b]pyrrol-4(1H)-one (3p)

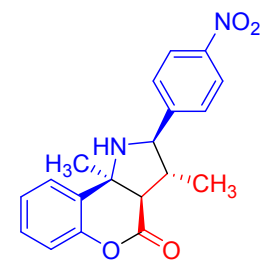

$3 p, 21 \%$ $97: 3$ er, $6: 1 \mathrm{dr}$

$\mathrm{NO}_{2}$ Yellow solid, 21\% yield, $7.2 \mathrm{mg}$; m.p. $129-131{ }^{\circ} \mathrm{C}$;

$\left[\underline{\underline{\alpha}]^{25} \mathbf{D}}=0.4\left(\mathrm{c}=0.5\right.\right.$ in $\left.\mathrm{CHCl}_{3}\right) ; 6: 1 \mathrm{dr}$ (reaction mixture);

$\underline{{ }^{1} \mathbf{H} \text { NMR }}\left(400 \mathrm{MHz}, \mathrm{CDCl}_{3}\right) \delta 8.08(\mathrm{~d}, J=8.8 \mathrm{~Hz}, 2 \mathrm{H}), 7.82-7.71$ (m, 1H), $7.34-7.27(\mathrm{~m}, 4 \mathrm{H}), 7.03(\mathrm{dt}, J=4.9,3.0 \mathrm{~Hz}, 1 \mathrm{H}), 4.22$ (d, $J=9.6 \mathrm{~Hz}, 1 \mathrm{H}), 2.86(\mathrm{~d}, J=12.1 \mathrm{~Hz}, 1 \mathrm{H}), 2.61(\mathrm{~s}, 1 \mathrm{H}), 2.24-2.06$ (m, 1H), $1.59(\mathrm{~s}, 4 \mathrm{H}), 1.16(\mathrm{~d}, J=6.5 \mathrm{~Hz}, 3 \mathrm{H})$.

${ }^{13} \mathrm{C} \mathrm{NMR}\left(101 \mathrm{MHz}, \mathrm{CDCl}_{3}\right) \delta 167.8,150.8,147.8,147.2,130.1,129.0,128.0,127.9$, $125.3,123.6,116.8,69.1,61.8,59.1,47.7,30.2,29.7$.

HRMS (ESI) calcd. for $\mathrm{C}_{19} \mathrm{H}_{18} \mathrm{~N}_{2} \mathrm{O}_{4} \mathrm{H}^{+}$: 339.1339, found : 339.1339;

UPLC analysis: 97:3 er (IA-U column, $25{ }^{\circ} \mathrm{C}$, hexane / $\mathrm{iPrOH}=85 / 15,0.5 \mathrm{~mL} /$ 
$\min , \lambda=254 \mathrm{~nm})$, Rt (minor) $=2.7 \mathrm{~min}$, Rt (major) $=2.9 \mathrm{~min}$

(2R,3S,3aR,9bS)-7-chloro-9b-methyl-2-(4-nitrophenyl)-3-phenyl-2,3,3a,9b-tetrah ydrochromeno[4,3-b]pyrrol-4(1H)-one (4a)

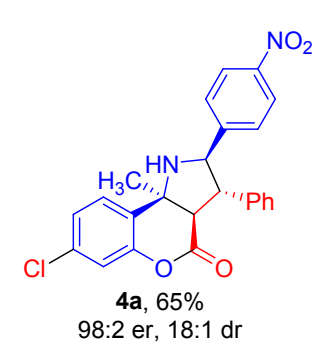

White solid, 65\% yield, $28.3 \mathrm{mg}$; m.p. $222-223{ }^{\circ} \mathrm{C}$;

$\lfloor\underline{\alpha}\rfloor^{25} \mathbf{D}=-14.7\left(\mathrm{c}=0.5\right.$ in $\left.\mathrm{CHCl}_{3}\right) ; 18: 1 \mathrm{dr}$ (reaction mixture);

$\underline{{ }^{1} \mathbf{H} \text { NMR }}\left(400 \mathrm{MHz}, \mathrm{CDCl}_{3}\right) \delta 7.95(\mathrm{~d}, J=8.7 \mathrm{~Hz}, 2 \mathrm{H}), 7.81(\mathrm{~d}, J$ $=8.3 \mathrm{~Hz}, 1 \mathrm{H}), 7.32-7.30(\mathrm{~m}, 4 \mathrm{H}), 7.09-7.05(\mathrm{~m}, 5 \mathrm{H}), 4.78(\mathrm{~d}, J=$

$9.9 \mathrm{~Hz}, 1 \mathrm{H}), 3.51(\mathrm{~d}, J=12.5 \mathrm{~Hz}, 1 \mathrm{H}), 3.18(\mathrm{dd}, J=12.3,10.0 \mathrm{~Hz}$,

1H), $2.76(\mathrm{~s}, 1 \mathrm{H}), 1.66(\mathrm{~s}, 3 \mathrm{H})$.

${ }^{13} \mathrm{C}$ NMR $\left(101 \mathrm{MHz}, \mathrm{CDCl}_{3}\right) \delta 166.1,149.8,148.3,147.1,135.3,134.3,129.4,129.0$, $128.5,128.3,127.9,127.4,125.6,123.4,117.2,68.8,62.1,58.7,58.6,29.8$.

HRMS (ESI) calcd. for $\mathrm{C}_{24} \mathrm{H}_{19} \mathrm{~N}_{2} \mathrm{O}_{4} \mathrm{ClH}^{+}: 435.1106$, found : 435.1104;

UPLC analysis: 98:2 er (IA-U column, $25{ }^{\circ} \mathrm{C}$, hexane / iPrOH $=80 / 20,0.5 \mathrm{~mL} /$ $\min , \lambda=254 \mathrm{~nm})$, Rt (minor) $=2.4 \mathrm{~min}$, Rt (major) $=2.9 \mathrm{~min}$.

\section{(2R,3S,3aR,9bS)-7-bromo-9b-methyl-2-(4-nitrophenyl)-3-phenyl-2,3,3a,9b-tetrah} ydrochromeno[4,3-b]pyrrol-4(1H)-one (4b)

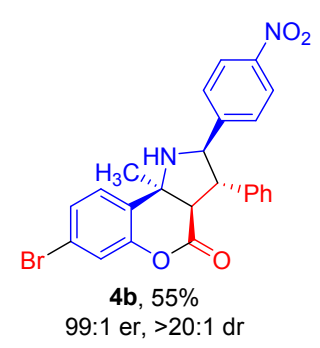

White solid, 55\% yield, $26.4 \mathrm{mg}$; m.p. 201-202 ${ }^{\circ} \mathrm{C}$;

$\underline{[\alpha]^{25} \mathbf{D}}=-14.0\left(\mathrm{c}=1.0\right.$ in $\left.\mathrm{CHCl}_{3}\right) ;>20: 1 \mathrm{dr}$ (reaction mixture);

1. $\mathbf{H}$ NMR $\left(400 \mathrm{MHz}, \mathrm{CDCl}_{3}\right) \delta$ 7.97-7.94 (m, 2H), $7.75(\mathrm{~d}, J=8.3$

$\mathrm{Hz}, 1 \mathrm{H}), 7.46(\mathrm{dd}, J=8.3,1.9 \mathrm{~Hz}, 1 \mathrm{H}), 7.31-7.28(\mathrm{~m}, 3 \mathrm{H}), 7.20(\mathrm{~d}$, $J=1.9 \mathrm{~Hz}, 1 \mathrm{H}), 7.12-7.04(\mathrm{~m}, 4 \mathrm{H}), 4.78(\mathrm{~d}, J=9.9 \mathrm{~Hz}, 1 \mathrm{H})$,

$3.51(\mathrm{~d}, J=12.5 \mathrm{~Hz}, 1 \mathrm{H}), 3.17(\mathrm{dd}, J=12.4,9.9 \mathrm{~Hz}, 1 \mathrm{H}), 2.71(\mathrm{~s}, 1 \mathrm{H}), 1.65$ (s, 3H).

${ }^{13} \mathrm{C} \mathrm{NMR}\left(101 \mathrm{MHz}, \mathrm{CDCl}_{3}\right) \delta 166.0,149.8,148.4,147.1,135.3,129.7,129.0,128.5$, $128.3,127.9,127.4,123.4,121.9,120.1,68.7,62.2,58.7,58.6,29.7$.

HRMS (ESI) calcd. for $\mathrm{C}_{24} \mathrm{H}_{19} \mathrm{~N}_{2} \mathrm{O}_{4} \mathrm{BrH}^{+}:$479.0601, found : 479.0600;

UPLC analysis: 99:1 er (IA-U column, $25{ }^{\circ} \mathrm{C}$, hexane / $\mathrm{iPrOH}=80 / 20,0.5 \mathrm{~mL} /$ $\min , \lambda=254 \mathrm{~nm})$, Rt (minor) $=2.5 \mathrm{~min}$, Rt (major) $=3.1 \mathrm{~min}$. 
(2R,3S,3aR,9bS)-7,9b-dimethyl-2-(4-nitrophenyl)-3-phenyl-2,3,3a,9b-tetrahydroc hromeno[4,3-b]pyrrol-4(1H)-one (4c)

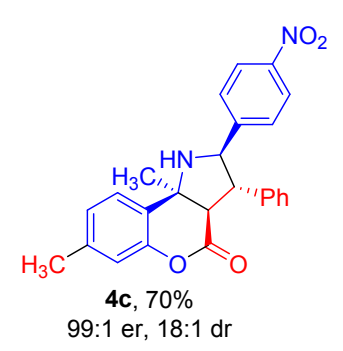

$\mathrm{NO}_{2} \quad$ White solid, $70 \%$ yield, $28.9 \mathrm{mg}$; m.p. $175-176^{\circ} \mathrm{C}$;

$\underline{[\alpha]^{25} \mathbf{D}}=-8.0\left(\mathrm{c}=1.0\right.$ in $\left.\mathrm{CHCl}_{3}\right) ; 18: 1 \mathrm{dr}$ (reaction mixture);

1․ NMR $\left(400 \mathrm{MHz}, \mathrm{CDCl}_{3}\right) \delta 7.93(\mathrm{~d}, J=8.7 \mathrm{~Hz}, 2 \mathrm{H}), 7.73(\mathrm{~d}$, $J=7.8 \mathrm{~Hz}, 1 \mathrm{H}), 7.30-7.25(\mathrm{~m}, 3 \mathrm{H}), 7.15-7.07$ (m, 5H), 6.84 (s, 1H), $4.76(\mathrm{~d}, J=9.8 \mathrm{~Hz}, 1 \mathrm{H}), 3.49$ (d, $J=12.4 \mathrm{~Hz}, 1 \mathrm{H}), 3.21$

(dd, $J=12.3,9.9 \mathrm{~Hz}, 1 \mathrm{H}), 2.71(\mathrm{~s}, 1 \mathrm{H}), 2.38(\mathrm{~s}, 3 \mathrm{H}), 1.64(\mathrm{~s}, 3 \mathrm{H})$.

${ }^{13} \mathrm{C} \mathrm{NMR}\left(101 \mathrm{MHz}, \mathrm{CDCl}_{3}\right) \delta 167.1,150.4,147.8,147.0,139.5,135.8,129.0,128.1$, $128.0,127.9,127.5,126.7,126.1,123.3,117.2,68.9,62.3,59.0,58.8,29.9,21.1$.

$\underline{\text { HRMS }}$ (ESI) calcd. for $\mathrm{C}_{25} \mathrm{H}_{22} \mathrm{~N}_{2} \mathrm{O}_{4} \mathrm{H}^{+}: 415.1652$, found : 415.1651;

UPLC analysis: 99:1 er (IA-U column, $25{ }^{\circ} \mathrm{C}$, hexane / $\mathrm{iPrOH}=80 / 20,0.5 \mathrm{~mL} /$ $\min , \lambda=254 \mathrm{~nm}$ ), Rt (minor) $=2.4 \mathrm{~min}$, Rt (major) $=3.2 \mathrm{~min}$.

(2R,3S,3aR,9bS)-7-methoxy-9b-methyl-2-(4-nitrophenyl)-3-phenyl-2,3,3a,9b-tetr ahydrochromeno $[4,3-b]$ pyrrol-4(1H)-one (4d)

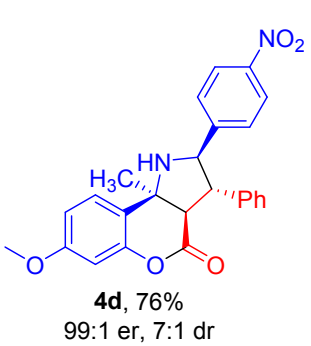

Light yellow solid, $76 \%$ yield, $32.8 \mathrm{mg}$; m.p. $85-87^{\circ} \mathrm{C}$;

$\left\lfloor\underline{\lfloor\alpha\rfloor^{25} \mathbf{D}}=-18.9\left(\mathrm{c}=0.5\right.\right.$ in $\left.\mathrm{CHCl}_{3}\right) ; 7: 1 \mathrm{dr}$ (reaction mixture);

$\underline{1 \mathbf{H} \text { NMR }}\left(400 \mathrm{MHz}, \mathrm{CDCl}_{3}\right) \delta 7.96-7.93(\mathrm{~m}, 2 \mathrm{H}), 7.74(\mathrm{~d}, J=$ $8.6 \mathrm{~Hz}, 1 \mathrm{H}), 7.30-7.27$ (m, 3H), $7.11-7.07$ (m, 4H), 6.89 (dd, $J$ $=8.6,2.5 \mathrm{~Hz}, 1 \mathrm{H}), 6.56(\mathrm{~d}, J=2.5 \mathrm{~Hz}, 1 \mathrm{H}), 4.76(\mathrm{~d}, J=9.8 \mathrm{~Hz}$,

1H), $3.82(\mathrm{~s}, 3 \mathrm{H}), 3.48(\mathrm{~d}, J=12.4 \mathrm{~Hz}, 1 \mathrm{H}), 3.23(\mathrm{dd}, J=12.3,9.9 \mathrm{~Hz}, 1 \mathrm{H}), 2.42$ (s, $1 \mathrm{H}), 1.64(\mathrm{~s}, 3 \mathrm{H})$.

${ }^{13} \mathrm{C} \mathrm{NMR}\left(101 \mathrm{MHz}, \mathrm{CDCl}_{3}\right) \delta 167.1,160.2,150.3,148.7,147.0,135.8,129.0,128.9$, $128.2,128.0,127.5,123.3,121.6,111.8,101.8,68.9,62.2,59.1,59.0,55.6,30.0$.

HRMS (ESI) calcd. for $\mathrm{C}_{25} \mathrm{H}_{22} \mathrm{~N}_{2} \mathrm{O}_{5} \mathrm{H}^{+}$: 431.1601, found : 431.1595;

UPLC analysis: 99:1 er (IA-U column, $25{ }^{\circ} \mathrm{C}$, hexane / $\mathrm{iPrOH}=90 / 10,0.5 \mathrm{~mL} /$ $\min , \lambda=254 \mathrm{~nm}), \operatorname{Rt}($ minor $)=5.7 \mathrm{~min}, \mathrm{Rt}($ major $)=8.0 \mathrm{~min}$. 
(2R,3S,3aR,9bS)-8-fluoro-9b-methyl-2-(4-nitrophenyl)-3-phenyl-2,3,3a,9b-tetrah ydrochromeno[4,3-b]pyrrol-4(1H)-one (4e)

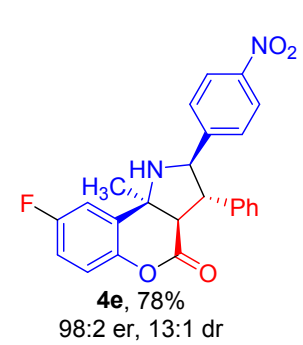

White solid, 78\% yield, $32.6 \mathrm{mg}$; m.p. $101-103{ }^{\circ} \mathrm{C}$;

$\lfloor\underline{[\alpha}\rfloor^{25} \mathbf{D}=-7.9\left(\mathrm{c}=1.0\right.$ in $\left.\mathrm{CHCl}_{3}\right) ; 13: 1 \mathrm{dr}$ (reaction mixture);

1H NMR $\left(400 \mathrm{MHz}, \mathrm{CDCl}_{3}\right) \delta 7.96-7.93(\mathrm{~m}, 2 \mathrm{H}), 7.59-7.56(\mathrm{~m}$, 1H), $7.32-7.27(\mathrm{~m}, 3 \mathrm{H}), 7.11-7.08(\mathrm{~m}, 4 \mathrm{H}), 7.02-7.00(\mathrm{~m}, 2 \mathrm{H})$, $4.79(\mathrm{~d}, J=9.9 \mathrm{~Hz}, 1 \mathrm{H}), 3.52(\mathrm{~d}, J=12.5 \mathrm{~Hz}, 1 \mathrm{H}), 3.18(\mathrm{dd}, J=$ $12.5,9.9 \mathrm{~Hz}, 1 \mathrm{H}), 2.82(\mathrm{~s}, 1 \mathrm{H}), 1.65(\mathrm{~s}, 3 \mathrm{H})$.

$\underline{{ }^{13} \mathbf{C ~ N M R}}\left(101 \mathrm{MHz}, \mathrm{CDCl}_{3}\right) \delta 166.4,159.7(\mathrm{~d}, J=244.1 \mathrm{~Hz}), 149.9,147.1,143.8(\mathrm{~d}$, $J=2.2 \mathrm{~Hz}), 135.4,132.0(\mathrm{~d}, J=6.6 \mathrm{~Hz}), 129.0,128.3,128.0,127.4,123.3,118.5(\mathrm{~d}$, $J=8.2 \mathrm{~Hz}), 116.1(\mathrm{~d}, J=24.0 \mathrm{~Hz}), 114.4(\mathrm{~d}, J=24.5 \mathrm{~Hz}), 68.7,62.4,58.6,58.4$, 29.6 .

$\underline{{ }^{19} \text { F NMR }}\left(377 \mathrm{MHz}, \mathrm{CDCl}_{3}\right) \delta-116.30$.

$\underline{\text { HRMS }}$ (ESI) calcd. for $\mathrm{C}_{24} \mathrm{H}_{19} \mathrm{~N}_{2} \mathrm{O}_{4} \mathrm{FH}^{+}$: 419.1402, found : 419.1400;

UPLC analysis: 98:2 er (IA-U column, $25{ }^{\circ} \mathrm{C}$, hexane / $\mathrm{iPrOH}=80 / 20,0.5 \mathrm{~mL} /$ $\min , \lambda=254 \mathrm{~nm})$, Rt (minor) $=2.3 \mathrm{~min}$, Rt (major) $=2.9 \mathrm{~min}$.

(2R,3S,3aR,9bS)-8-chloro-9b-methyl-2-(4-nitrophenyl)-3-phenyl-2,3,3a,9b-tetrah ydrochromeno[4,3-b]pyrrol-4(1H)-one (4f)

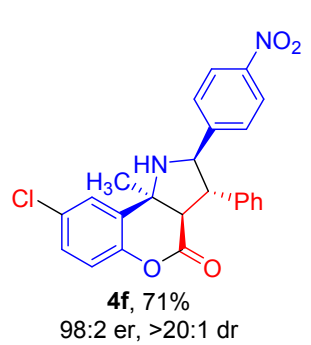

White solid, 71\% yield, $30.9 \mathrm{mg}$; m.p. $89-91{ }^{\circ} \mathrm{C}$;

$\underline{\lfloor\alpha\rfloor^{25} \mathbf{D}}=82.2\left(\mathrm{c}=1.0\right.$ in $\left.\mathrm{CHCl}_{3}\right) ;>20: 1 \mathrm{dr}$ (reaction mixture);

$\underline{{ }^{1} \mathbf{H ~ N M R}}\left(400 \mathrm{MHz}, \mathrm{CDCl}_{3}\right) \delta 7.98-7.95(\mathrm{~m}, 2 \mathrm{H}), 7.85-7.83$ (m, 1H), $7.32-7.26(\mathrm{~m}, 4 \mathrm{H}), 7.11-7.07(\mathrm{~m}, 4 \mathrm{H}), 6.99(\mathrm{~d}, J=$

$8.7 \mathrm{~Hz}, 1 \mathrm{H}), 4.78(\mathrm{~d}, J=9.9 \mathrm{~Hz}, 1 \mathrm{H}), 3.51(\mathrm{~d}, J=12.5 \mathrm{~Hz}, 1 \mathrm{H})$,

$3.17(\mathrm{dd}, J=12.4,9.9 \mathrm{~Hz}, 1 \mathrm{H}), 2.76(\mathrm{~s}, 1 \mathrm{H}), 1.67$ (s, 3H).

13C NMR $\left(101 \mathrm{MHz}, \mathrm{CDCl}_{3}\right) \delta 166.2,149.7,147.2,146.5,135.4,131.7,130.4,129.3$, 129.1, 128.4, 128.1, 128.0, 127.4, 123.4, 118.5, 68.8, 62.3, 58.7, 58.5, 29.7.

HRMS (ESI) calcd. for $\mathrm{C}_{24} \mathrm{H}_{19} \mathrm{~N}_{2} \mathrm{O}_{4} \mathrm{ClH}^{+}$: 435.1106, found : 435.1100;

UPLC analysis: 98:2 er (IA-U column, $25{ }^{\circ} \mathrm{C}$, hexane / $\mathrm{iPrOH}=80 / 20,0.5 \mathrm{~mL} /$ $\min , \lambda=254 \mathrm{~nm}), \mathrm{Rt}($ minor $)=2.2 \mathrm{~min}, \mathrm{Rt}($ major $)=2.7 \mathrm{~min}$. 
(2R,3S,3aR,9bS)-8-bromo-9b-methyl-2-(4-nitrophenyl)-3-phenyl-2,3,3a,9b-tetrah ydrochromeno[4,3-b]pyrrol-4(1H)-one $(4 \mathrm{~g})$

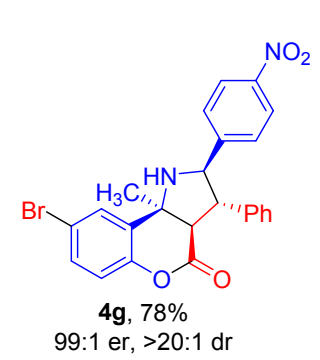

White solid, 78\% yield, $37.6 \mathrm{mg}$; m.p. $105-106^{\circ} \mathrm{C}$;

$[\alpha]^{25} \mathrm{D}=63.6\left(\mathrm{c}=0.5\right.$ in $\left.\mathrm{CHCl}_{3}\right) ;>20: 1 \mathrm{dr}$ (reaction mixture);

$\underline{1 \mathbf{H} \text { NMR }}\left(400 \mathrm{MHz}, \mathrm{CDCl}_{3}\right) \delta 8.00(\mathrm{~d}, J=2.4 \mathrm{~Hz}, 1 \mathrm{H}), 7.99-$ $7.96(\mathrm{~m}, 2 \mathrm{H}), 7.43(\mathrm{dd}, J=8.6,2.4 \mathrm{~Hz}, 1 \mathrm{H}), 7.32-7.28(\mathrm{~m}, 3 \mathrm{H})$, $7.11-7.05(\mathrm{~m}, 4 \mathrm{H}), 6.93(\mathrm{~d}, J=8.6 \mathrm{~Hz}, 1 \mathrm{H}), 4.78(\mathrm{~d}, J=9.8 \mathrm{~Hz}$,

1H), $3.50(\mathrm{~d}, J=12.5 \mathrm{~Hz}, 1 \mathrm{H}), 3.17(\mathrm{dd}, J=12.4,9.9 \mathrm{~Hz}, 1 \mathrm{H}), 2.78(\mathrm{~s}, 1 \mathrm{H}), 1.67(\mathrm{~s}$, $3 \mathrm{H})$.

${ }^{13} \mathrm{C}$ NMR $\left(101 \mathrm{MHz}, \mathrm{CDCl}_{3}\right) \delta 166.1,149.7,147.2,147.0,135.4,132.2,132.0,131.2$, $129.1,128.4,127.9,127.4,123.4,118.8,117.8,68.7,62.3,58.64$ 58.5, 29.6.

HRMS (ESI) calcd. for $\mathrm{C}_{24} \mathrm{H}_{19} \mathrm{~N}_{2} \mathrm{O}_{4} \mathrm{BrH}^{+}:$479.0601, found : 479.0598;

UPLC analysis: 99:1 er (IA-U column, $25{ }^{\circ} \mathrm{C}$, hexane / iPrOH $=80 / 20,0.5 \mathrm{~mL} /$ $\min , \lambda=254 \mathrm{~nm})$, Rt (minor) $=2.3 \mathrm{~min}$, Rt (major) $=2.7 \mathrm{~min}$.

\section{(2R,3S,3aR,9bS)-9b-methyl-8-nitro-2-(4-nitrophenyl)-3-phenyl-2,3,3a,9b-tetrahy} drochromeno[4,3-b]pyrrol-4(1H)-one $(4 \mathrm{~h})$

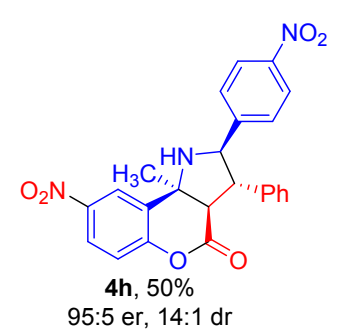

$$
\begin{aligned}
& \text { White solid, } 50 \% \text { yield, } 22.3 \mathrm{mg} \text {; m.p. } 124-125^{\circ} \mathrm{C} \text {; } \\
& \underline{[\alpha]^{25} \mathbf{D}}=33.5\left(\mathrm{c}=0.3 \text { in } \mathrm{CHCl}_{3}\right) ; 14: 1 \mathrm{dr} \text { (reaction mixture); }
\end{aligned}
$$

$\underline{{ }^{1} \mathbf{H} \text { NMR }}\left(400 \mathrm{MHz}, \mathrm{CDCl}_{3}\right) \delta 8.82(\mathrm{~d}, J=2.7 \mathrm{~Hz}, 1 \mathrm{H}), 8.21(\mathrm{dd}$, $J=8.9,2.8 \mathrm{~Hz}, 1 \mathrm{H}), 7.94(\mathrm{~d}, J=8.8 \mathrm{~Hz}, 2 \mathrm{H}), 7.34-7.32(\mathrm{~m}, 3 \mathrm{H})$, $7.19(\mathrm{~d}, J=8.9 \mathrm{~Hz}, 1 \mathrm{H}), 7.11-7.08(\mathrm{~m}, 4 \mathrm{H}), 4.85(\mathrm{~d}, J=9.9 \mathrm{~Hz}$,

1H), $3.60(\mathrm{~d}, J=12.5 \mathrm{~Hz}, 1 \mathrm{H}), 3.14(\mathrm{dd}, J=12.4,9.9 \mathrm{~Hz}, 1 \mathrm{H}), 2.90(\mathrm{~s}, 1 \mathrm{H}), 1.75(\mathrm{~s}$, $3 \mathrm{H})$.

$\underline{{ }^{13} \mathbf{C ~ N M R}}\left(101 \mathrm{MHz}, \mathrm{CDCl}_{3}\right) \delta 165.1,152.2,149.1,147.3,145.1,134.9,131.7,129.2$, $128.6,128.0,127.2,124.9,124.7,123.5,118.2,68.5,62.1,58.7,58.2,29.7$.

HRMS (ESI) calcd. for $\mathrm{C}_{24} \mathrm{H}_{19} \mathrm{~N}_{3} \mathrm{O}_{6} \mathrm{H}^{+}$: 446.1347, found : 446.1350;

UPLC analysis: 95:5 er (IA-U column, $25{ }^{\circ} \mathrm{C}$, hexane / $\mathrm{iPrOH}=80 / 20,0.5 \mathrm{~mL} /$ $\min , \lambda=254 \mathrm{~nm}), \mathrm{Rt}($ minor $)=3.6 \mathrm{~min}, \mathrm{Rt}($ major $)=4.3 \mathrm{~min}$. 
(2R,3S,3aR,9bS)-8,9b-dimethyl-2-(4-nitrophenyl)-3-phenyl-2,3,3a,9b-tetrahydroc hromeno[4,3-b]pyrrol-4(1H)-one (4i)

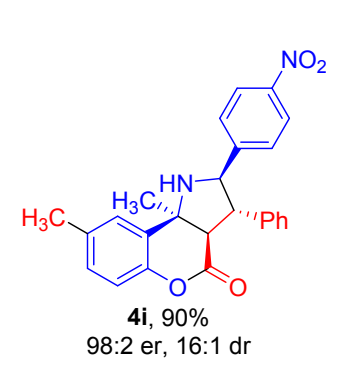

White solid, 90\% yield, $37.4 \mathrm{mg}$; m.p. $88-90^{\circ} \mathrm{C}$;

$\left\lfloor\underline{[\alpha\rfloor^{25} \mathbf{D}}=34.2\left(\mathrm{c}=0.5\right.\right.$ in $\left.\mathrm{CHCl}_{3}\right) ; 16: 1 \mathrm{dr}$ (reaction mixture);

$\underline{\mathbf{H} \text { NMR }}\left(400 \mathrm{MHz}, \mathrm{CDCl}_{3}\right) \delta 7.96-7.93(\mathrm{~m}, 2 \mathrm{H}), 7.64(\mathrm{~d}, J=$ $1.8 \mathrm{~Hz}, 1 \mathrm{H}), 7.32-7.26(\mathrm{~m}, 3 \mathrm{H}), 7.13-7.07$ (m, 5H), $6.92(\mathrm{~d}, J$ $=8.2 \mathrm{~Hz}, 1 \mathrm{H}), 4.76(\mathrm{~d}, J=9.8 \mathrm{~Hz}, 1 \mathrm{H}), 3.48(\mathrm{~d}, J=12.4 \mathrm{~Hz}, 1 \mathrm{H})$,

$3.21(\mathrm{dd}, J=12.4,9.8 \mathrm{~Hz}, 1 \mathrm{H}), 2.45(\mathrm{~s}, 3 \mathrm{H}), 1.65$ (s, 3H).

${ }^{13} \mathrm{C} \mathrm{NMR}\left(101 \mathrm{MHz}, \mathrm{CDCl}_{3}\right) \delta 167.1,150.3,147.1,145.9,135.9,134.9,129.8,129.3$, 129.0, 128.3, 128.2, 128.0, 127.6, 123.3, 116.7, 68.9, 62.5, 59.0, 58.8, 29.9, 21.1.

HRMS (ESI) calcd. for $\mathrm{C}_{25} \mathrm{H}_{22} \mathrm{~N}_{2} \mathrm{O}_{4} \mathrm{H}^{+}$: 415.1652, found : 415.1651;

UPLC analysis: 98:2 er (IA-U column, $25{ }^{\circ} \mathrm{C}$, hexane / $\mathrm{iPrOH}=80 / 20,0.5 \mathrm{~mL} /$ $\min , \lambda=254 \mathrm{~nm}$ ), Rt (minor) $=2.1 \mathrm{~min}, \mathrm{Rt}($ major $)=2.6 \mathrm{~min}$.

(2R,3S,3aR,9bS)-9-methoxy-9b-methyl-2-(4-nitrophenyl)-3-phenyl-2,3,3a,9b-tetr ahydrochromeno[4,3-b]pyrrol-4(1H)-one $(4 \mathrm{j})$

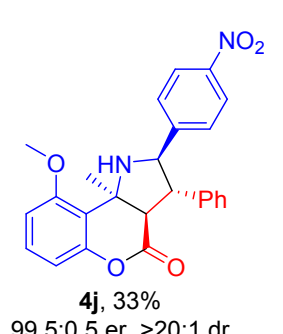

White solid, $73 \%$ yield, $14.1 \mathrm{mg}$; m.p. $95-97{ }^{\circ} \mathrm{C}$;

$\underline{[\alpha\rfloor^{25} \mathbf{D}}=-11.4\left(\mathrm{c}=0.4\right.$ in $\left.\mathrm{CHCl}_{3}\right) ;>20: 1 \mathrm{dr}$ (reaction mixture);

$\underline{{ }^{1} \mathbf{H} \text { NMR }}\left(400 \mathrm{MHz}, \mathrm{CDCl}_{3}\right) \delta 8.00-7.96(\mathrm{~m}, 2 \mathrm{H}), 7.34(\mathrm{t}, J=8.3$

$\mathrm{Hz}, 1 \mathrm{H}), 7.29-7.25$ (m, 3H), 7.11-7.09 (m, 2H), $6.99-6.94$ (m, 2H), 99.5:0.5 er, $>20: 1 \mathrm{dr}$

$6.85(\mathrm{dd}, J=8.4,0.8 \mathrm{~Hz}, 1 \mathrm{H}), 6.79(\mathrm{dd}, J=8.3,1.0 \mathrm{~Hz}, 1 \mathrm{H}), 4.60$ $(\mathrm{d}, J=9.3 \mathrm{~Hz}, 1 \mathrm{H}), 3.92(\mathrm{~s}, 3 \mathrm{H}), 3.71(\mathrm{~s}, 1 \mathrm{H}), 3.53(\mathrm{~d}, J=12.3 \mathrm{~Hz}, 1 \mathrm{H}), 3.38(\mathrm{dd}, J=$ 12.2, $9.3 \mathrm{~Hz}, 1 \mathrm{H}), 1.72(\mathrm{~s}, 3 \mathrm{H})$.

${ }^{13} \mathrm{C} \mathrm{NMR}\left(101 \mathrm{MHz}, \mathrm{CDCl}_{3}\right) \delta 167.0,158.0,150.3,148.9,147.3,136.0,129.4,128.9$, 128.1, 127.8, 127.5, 123.8, 117.2, 110.4, 107.6, 69.1, 62.7, 58.5, 57.9, 56.0, 28.2.

$\underline{\text { HRMS }}$ (ESI) calcd. for $\mathrm{C}_{25} \mathrm{H}_{22} \mathrm{~N}_{2} \mathrm{O}_{5} \mathrm{H}^{+}: 431.1601$, found : 431.1602;

UPLC analysis: 99.5:0.5 er (IA-U column, $25^{\circ} \mathrm{C}$, hexane / $\mathrm{iPrOH}=90 / 10,0.5 \mathrm{~mL} /$ $\min , \lambda=254 \mathrm{~nm}$ ), Rt (minor) $=3.5 \mathrm{~min}$, Rt (major) $=4.0 \mathrm{~min}$. 
(2R,3S,3aR,9bS)-9b-ethyl-2-(4-nitrophenyl)-3-phenyl-2,3,3a,9b-tetrahydrochrom eno[4,3-b]pyrrol-4(1H)-one (4k)

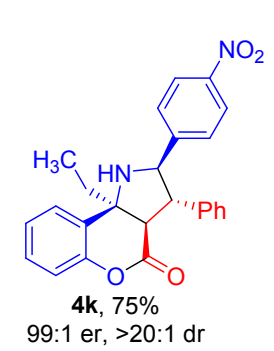

White solid, 75\% yield, $31.1 \mathrm{mg}$; m.p. $72-74{ }^{\circ} \mathrm{C}$;

$\lfloor\boldsymbol{\alpha}\rfloor^{25} \mathbf{D}=-2.2\left(\mathrm{c}=0.5\right.$ in $\left.\mathrm{CHCl}_{3}\right) ;>20: 1 \mathrm{dr}$ (reaction mixture);

1․ NMR $\left(400 \mathrm{MHz}, \mathrm{CDCl}_{3}\right) \delta 7.94-7.91(\mathrm{~m}, 2 \mathrm{H}), 7.81-7.79(\mathrm{~m}$, 1H), $7.36-7.32(\mathrm{~m}, 2 \mathrm{H}), 7.31-7.26(\mathrm{~m}, 3 \mathrm{H}), 7.09-7.02(\mathrm{~m}, 5 \mathrm{H})$, $4.74(\mathrm{~d}, J=9.9 \mathrm{~Hz}, 1 \mathrm{H}), 3.49(\mathrm{~d}, J=12.4 \mathrm{~Hz}, 1 \mathrm{H}), 3.18(\mathrm{dd}, J=12.4$, $10.0 \mathrm{~Hz}, 1 \mathrm{H}), 2.75(\mathrm{~s}, 1 \mathrm{H}), 2.06-1.88(\mathrm{~m}, 2 \mathrm{H}), 0.89$ (t, J=7.5 Hz, 3H).

${ }^{13} \mathrm{C}$ NMR $\left(101 \mathrm{MHz}, \mathrm{CDCl}_{3}\right) \delta 167.2,150.2,149.1,147.1,135.6,129.2,129.0,128.7$, $128.2,128.2,128.1,127.5,125.2,123.3,116.7,68.7,66.1,58.8,56.8,36.6,8.8$.

HRMS (ESI) calcd. for $\mathrm{C}_{25} \mathrm{H}_{22} \mathrm{~N}_{2} \mathrm{O}_{4} \mathrm{H}^{+}:$415.1652, found : 415.1650;

UPLC analysis: 99:1 er (IA-U column, $25{ }^{\circ} \mathrm{C}$, hexane / $\mathrm{iPrOH}=80 / 20,0.5 \mathrm{~mL} /$ $\min , \lambda=254 \mathrm{~nm})$, Rt (minor) $=2.2 \mathrm{~min}$, Rt (major) $=2.9 \mathrm{~min}$.

\section{(2R,3S,3aR,9bR)-2-(4-nitrophenyl)-3,9b-diphenyl-2,3,3a,9b-tetrahydrochromeno[}

\section{4,3-b]pyrrol-4(1H)-one (4l)}

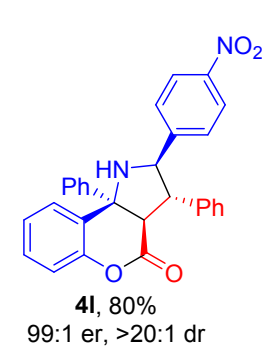

White solid, 80\% yield, 36.9 mg; m.p. $108-109^{\circ} \mathrm{C}$;

$[\alpha]^{25} \mathrm{D}=42.8\left(\mathrm{c}=0.5\right.$ in $\left.\mathrm{CHCl}_{3}\right) ;>20: 1 \mathrm{dr}$ (reaction mixture);

1H NMR $\left(400 \mathrm{MHz}, \mathrm{CDCl}_{3}\right) \delta 7.99-7.97(\mathrm{~m}, 2 \mathrm{H}), 7.84(\mathrm{dd}, J=7.4$, $2.0 \mathrm{~Hz}, 1 \mathrm{H}), 7.40-7.34(\mathrm{~m}, 6 \mathrm{H}), 7.32-7.27(\mathrm{~m}, 4 \mathrm{H}), 7.18-7.16(\mathrm{~m}$, 2H), $7.12-7.08(\mathrm{~m}, 3 \mathrm{H}), 4.93(\mathrm{~d}, J=9.9 \mathrm{~Hz}, 1 \mathrm{H}), 3.95(\mathrm{~d}, J=12.1$

$\mathrm{Hz}, 1 \mathrm{H}), 3.37$ (dd, $J=12.1,9.9 \mathrm{~Hz}, 1 \mathrm{H}), 3.25$ (s, 1H).

${ }^{13} \mathrm{C}$ NMR $\left(101 \mathrm{MHz}, \mathrm{CDCl}_{3}\right) \delta 166.5,149.6,148.7,147.2,144.5,135.4,129.6,129.4$, $129.3,129.0,128.6,128.3,128.2,128.0,127.6,125.5,125.4,123.4,117.1,69.4,67.8$, $59.6,58.8$.

$\underline{\text { HRMS }}$ (ESI) calcd. for $\mathrm{C}_{29} \mathrm{H}_{22} \mathrm{~N}_{2} \mathrm{O}_{4} \mathrm{H}^{+}$: 463.1652, found : 463.1653;

UPLC analysis: 99:1 er (IA-U column, 25 oC, hexane / iPrOH = 80 / 20, $0.5 \mathrm{~mL} /$ $\min , \lambda=254 \mathrm{~nm}$ ), Rt (minor) $=6.9 \mathrm{~min}$, Rt (major) $=9.0 \mathrm{~min}$. 
methyl(2S,3R,4S,5R)-2-(2-hydroxyphenyl)-2-methyl-5-(4-nitrophenyl)-4-phenylp yrrolidine-3-carboxylate (5)

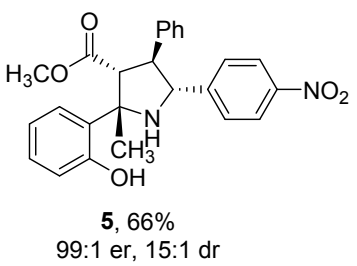

White solid, 66\% yield, $36.9 \mathrm{mg}$; m.p. $166-168^{\circ} \mathrm{C}$;

$\left\lfloor\underline{[\alpha\rfloor^{25} \mathbf{D}}=-21.4\left(\mathrm{c}=0.5 \mathrm{in} \mathrm{CHCl}_{3}\right) ; 15: 1 \mathrm{dr}\right.$ (reaction mixture); ${ }^{1} \mathrm{H}$ NMR $\left(400 \mathrm{MHz}, \mathrm{CDCl}_{3}\right) \delta 8.14-8.12(\mathrm{~m}, 2 \mathrm{H}), 7.46(\mathrm{~d}, J$ $=8.7 \mathrm{~Hz}, 2 \mathrm{H}), 7.26-7.11(\mathrm{~m}, 7 \mathrm{H}), 6.86-6.77(\mathrm{~m}, 2 \mathrm{H}), 4.70$ $(\mathrm{d}, J=11.0 \mathrm{~Hz}, 1 \mathrm{H}), 4.05$ (t, $J=10.9 \mathrm{~Hz}, 1 \mathrm{H}), 3.36$ (d, $J=10.7 \mathrm{~Hz}, 1 \mathrm{H}), 3.13(\mathrm{~s}, 3 \mathrm{H})$, $2.11(\mathrm{~s}, 3 \mathrm{H})$.

$\underline{{ }^{13} \mathbf{C ~ N M R}}\left(101 \mathrm{MHz}, \mathrm{CDCl}_{3}\right) \delta 170.5,158.6,147.9,145.7,137.0,129.8,129.0$, $128.2,127.8,127.7,124.7,124.0,118.5,117.7,67.2,66.2,65.3,56.1,51.7,30.6$.

HRMS $(E S I)$ calcd. for $\mathrm{C}_{25} \mathrm{H}_{25} \mathrm{~N}_{2} \mathrm{O}_{5} \mathrm{H}^{+}: 433.1758$, found : 433.1754;

HPLC analysis: 99:1 er (IA column, $25^{\circ} \mathrm{C}$, hexane / $\mathrm{iPrOH}=80 / 20,0.5 \mathrm{~mL} / \mathrm{min}$, $\lambda=254 \mathrm{~nm}), \operatorname{Rt}($ minor $)=24.0 \mathrm{~min}, \mathrm{Rt}($ major $)=20.1 \mathrm{~min}$.

(2R,3S,3aR,9bS)-2-(4-aminophenyl)-9b-methyl-3-phenyl-2,3,3a,9b-tetrahydrochr omeno[4,3-b]pyrrol-4(1H)-one (6)

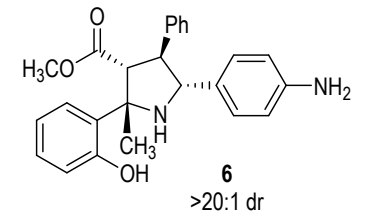

White solid, 96\% yield, $200 \mathrm{mg}$; m.p. $94-96^{\circ} \mathrm{C}$;

$\underline{[\alpha]^{25} \mathbf{D}}=-74.9\left(\mathrm{c}=0.5 \mathrm{in} \mathrm{CHCl}_{3}\right) ;>20: 1 \mathrm{dr}$ (reaction mixture);

1H NMR $\left(400 \mathrm{MHz}, \mathrm{CDCl}_{3}\right) \delta 7.23-7.11(\mathrm{~m}, 9 \mathrm{H}), 6.82(\mathrm{dd}, J$ $=7.9,0.8 \mathrm{~Hz}, 1 \mathrm{H}), 6.78-6.72(\mathrm{~m}, 1 \mathrm{H}), 6.61-6.55(\mathrm{~m}, 2 \mathrm{H}), 4.47(\mathrm{~d}, J=11.1 \mathrm{~Hz}$, 1H), 4.04 (t, $J=11.0 \mathrm{~Hz}, 1 \mathrm{H}), 3.29(\mathrm{~d}, J=10.9 \mathrm{~Hz}, 1 \mathrm{H}), 3.11(\mathrm{~s}, 3 \mathrm{H}), 2.04(\mathrm{~s}, 3 \mathrm{H})$.

$\underline{{ }^{13} \mathbf{C ~ N M R}}\left(101 \mathrm{MHz}, \mathrm{CDCl}_{3}\right) \delta 170.9,159.1,146.4,138.2,129.4,128.6,128.5$, $127.9,127.6,127.6,127.1,125.2,118.1,117.5,115.3,66.3,66.2,65.4,55.1,51.5$, 30.5 .

HRMS (ESI) calcd. for $\mathrm{C}_{25} \mathrm{H}_{26} \mathrm{~N}_{2} \mathrm{O}_{3} \mathrm{H}^{+}$: 403.2016, found : 403.2014; 


\section{1-(3,5-bis(trifluoromethyl)phenyl)-3-(4-((2R,3S,3aR,9bS)-9b-methyl-4-oxo-3-phe}

nyl-1,2,3,3a,4,9b-hexahydrochromeno[4,3-b]pyrrol-2-yl)phenyl)thiourea (7)

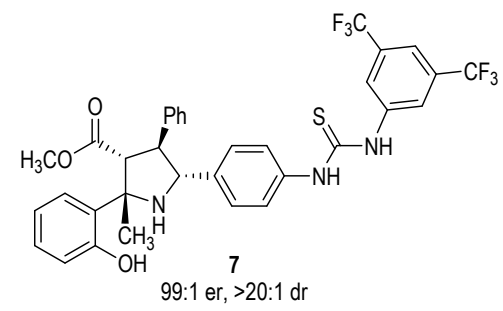

White solid, 72\% yield, $61.5 \mathrm{mg}$; m.p. $135-136{ }^{\circ} \mathrm{C}$; $\underline{[\alpha]^{25} \mathbf{D}}=-81.4\left(\mathrm{c}=0.5\right.$ in $\left.\mathrm{CHCl}_{3}\right) ;>20: 1 \mathrm{dr}($ reaction mixture);

1H NMR $\left(400 \mathrm{MHz}, \mathrm{CDCl}_{3}\right) \delta 8.97(\mathrm{~s}, 1 \mathrm{H}), 8.34(\mathrm{~s}$, 1H), $7.96(\mathrm{~s}, 2 \mathrm{H}), 7.59$ (s, 1H), $7.42(\mathrm{~d}, J=8.1 \mathrm{~Hz}, 2 \mathrm{H}), 7.17$ (dd, $J=17.4,7.9 \mathrm{~Hz}$, 4H), $7.05(\mathrm{dt}, J=20.3,7.6 \mathrm{~Hz}, 3 \mathrm{H}), 6.89$ (d, $J=7.4 \mathrm{~Hz}, 2 \mathrm{H}), 6.78(\mathrm{dd}, J=7.3,4.8$ $\mathrm{Hz}, 2 \mathrm{H}), 4.60(\mathrm{~d}, J=11.1 \mathrm{~Hz}, 1 \mathrm{H}), 3.82(\mathrm{t}, J=11.1 \mathrm{~Hz}, 1 \mathrm{H}), 3.36(\mathrm{~d}, J=11.1 \mathrm{~Hz}$, $1 \mathrm{H}), 3.11(\mathrm{~s}, 3 \mathrm{H}), 2.06(\mathrm{~s}, 3 \mathrm{H})$.

${ }^{13} \mathrm{C} \mathrm{NMR}\left(101 \mathrm{MHz}, \mathrm{CDCl}_{3}\right) \delta 180.3,172.2,158.5,140.3,137.7,136.8,131.5$ (q, $J=$ $33.5 \mathrm{~Hz}), 129.9,129.0,127.9,127.7,127.4,125.6,125.5,124.5,123.5(\mathrm{~d}, J=2.6 \mathrm{~Hz})$, 121.8, 119.1, $118.2(\mathrm{~d}, J=3.7 \mathrm{~Hz}), 117.6,66.1,65.7,65.3,55.9,51.8,29.9$.

$\underline{{ }^{19} \mathbf{F ~ N M R}}\left(377 \mathrm{MHz}, \mathrm{CDCl}_{3}\right) \delta-62.87$.

HRMS (ESI) calcd. for $\mathrm{C}_{34} \mathrm{H}_{29} \mathrm{~N}_{3} \mathrm{O}_{3} \mathrm{~F}_{6} \mathrm{SH}^{+}:$674.1907, found : 674.1902;

HPLC analysis: 99:1 er (IA column, $25^{\circ} \mathrm{C}$, hexane / $\mathrm{iPrOH}=80 / 20,0.5 \mathrm{~mL} / \mathrm{min}$, $\lambda=254 \mathrm{~nm}), \operatorname{Rt}($ minor $)=12.8 \mathrm{~min}, \mathrm{Rt}($ major $)=35.1 \mathrm{~min}$. 
IX. ${ }^{1} \mathrm{H}$ NMR, ${ }^{13} \mathrm{C}$ NMR, ${ }^{19}$ F NMR and HPLC spectra

(E)-2-(1-((4-nitrobenzyl)imino)ethyl)phenol (2a):

官

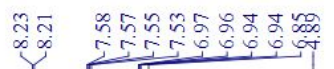<smiles>CC(=NCc1ccc([N+](=O)[O-])cc1)c1ccccc1O</smiles>

$2 a$

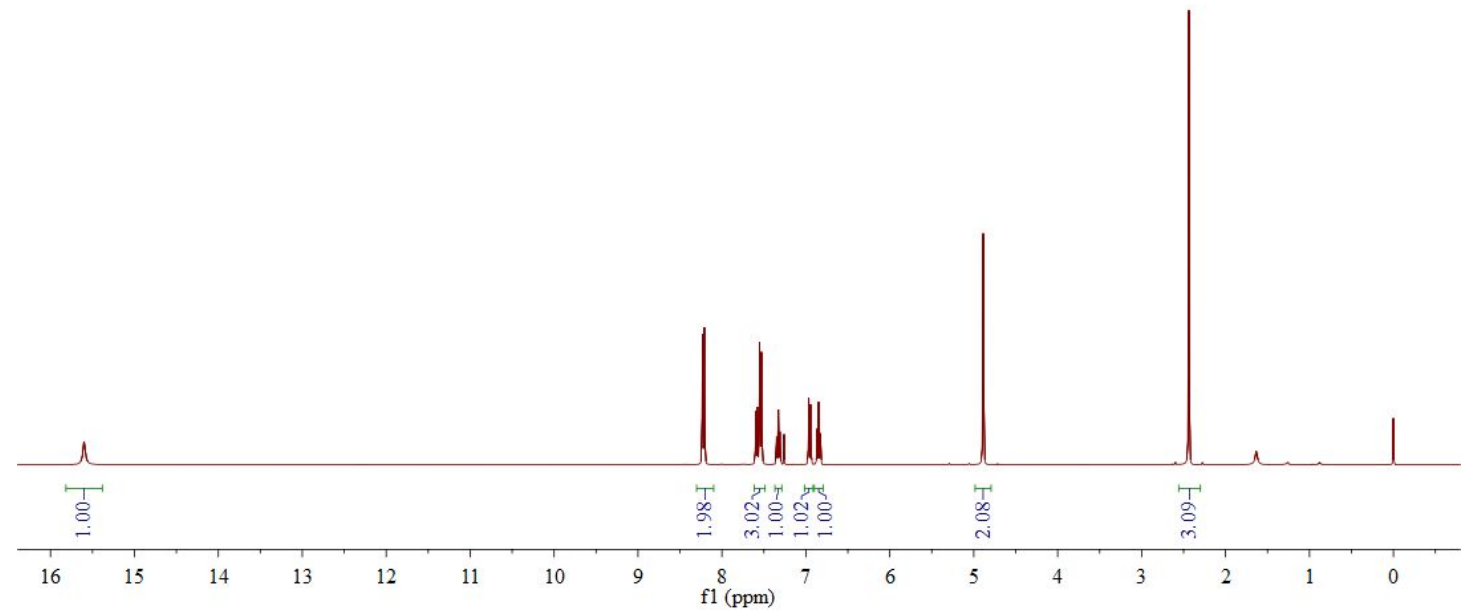

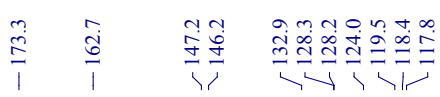
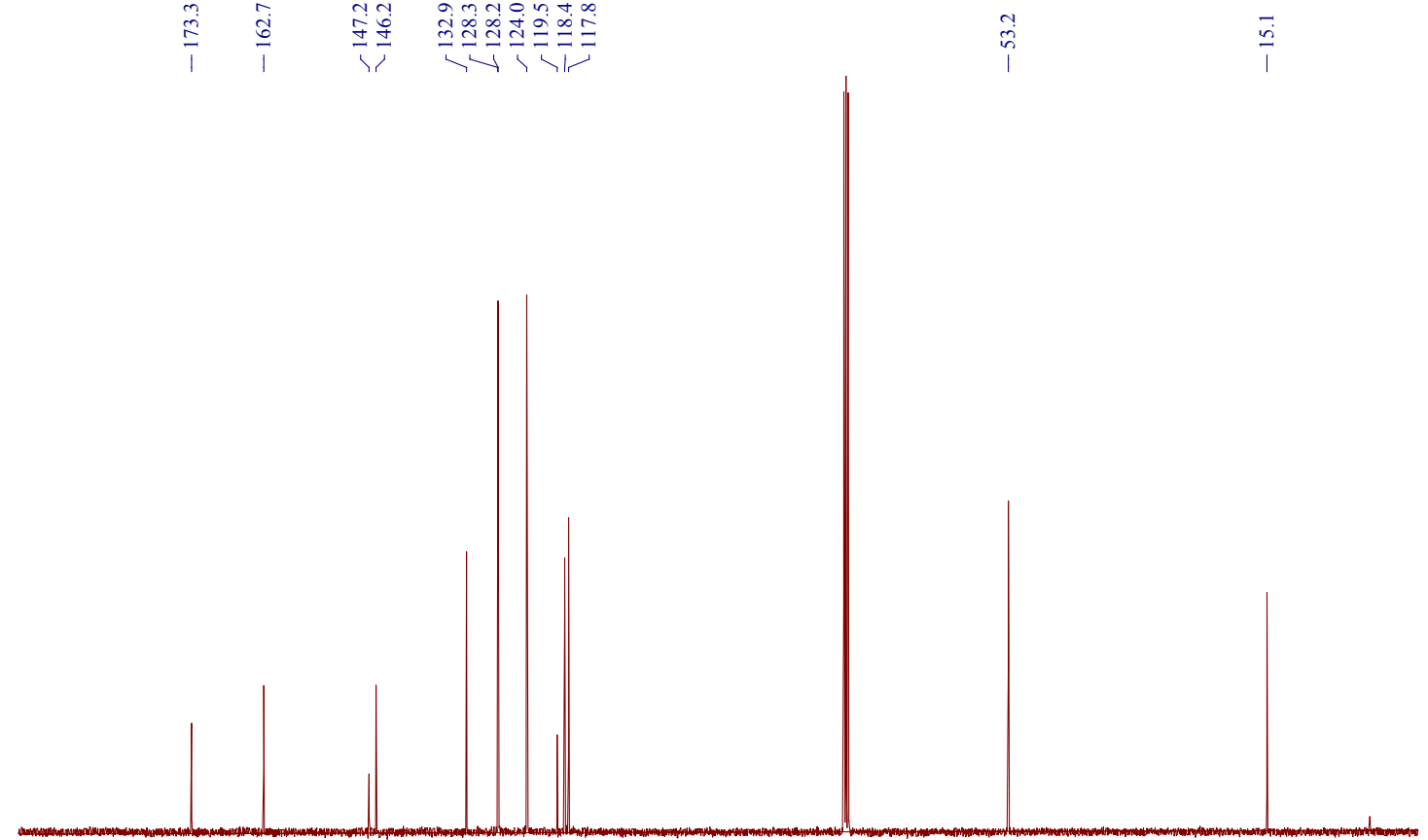

$\begin{array}{llllllllll}190 & 180 & 170 & 160 & 150 & 140 & 130 & 120 & 110 & \begin{array}{l}100 \\ \mathrm{fl}(\mathrm{ppm})\end{array}\end{array}$ 
(E)-4-chloro-2-(1-((4-nitrobenzyl)imino)ethyl)phenol (2b):

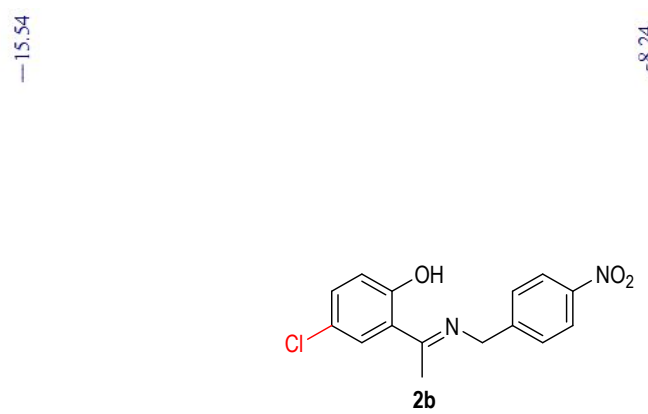

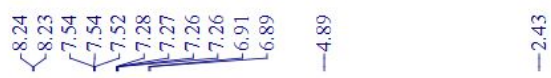

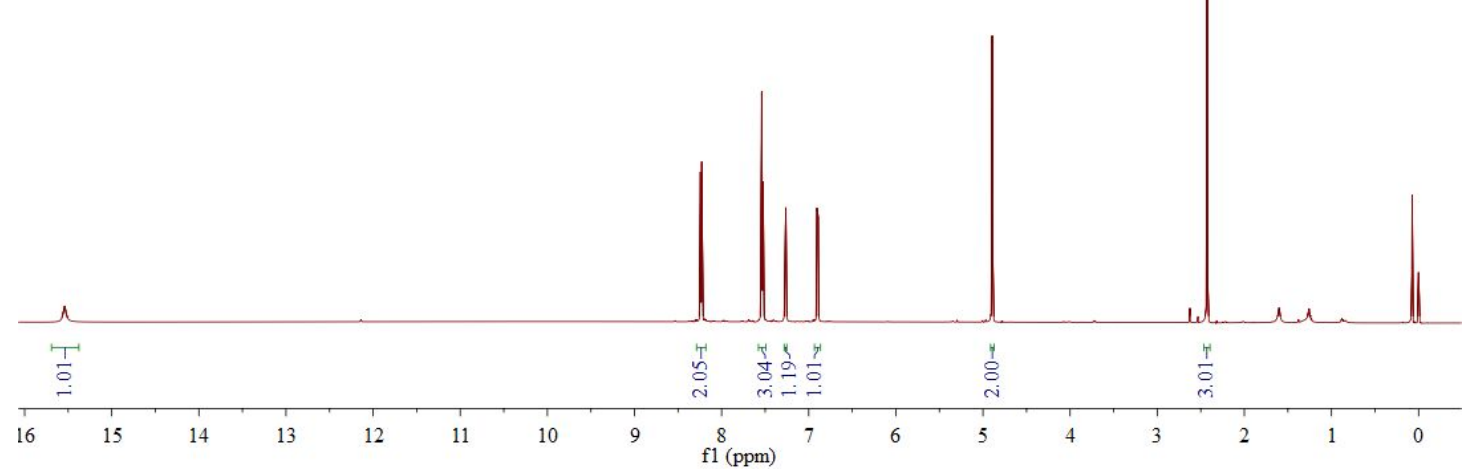

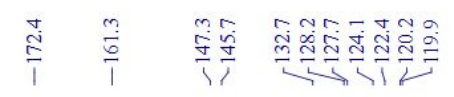

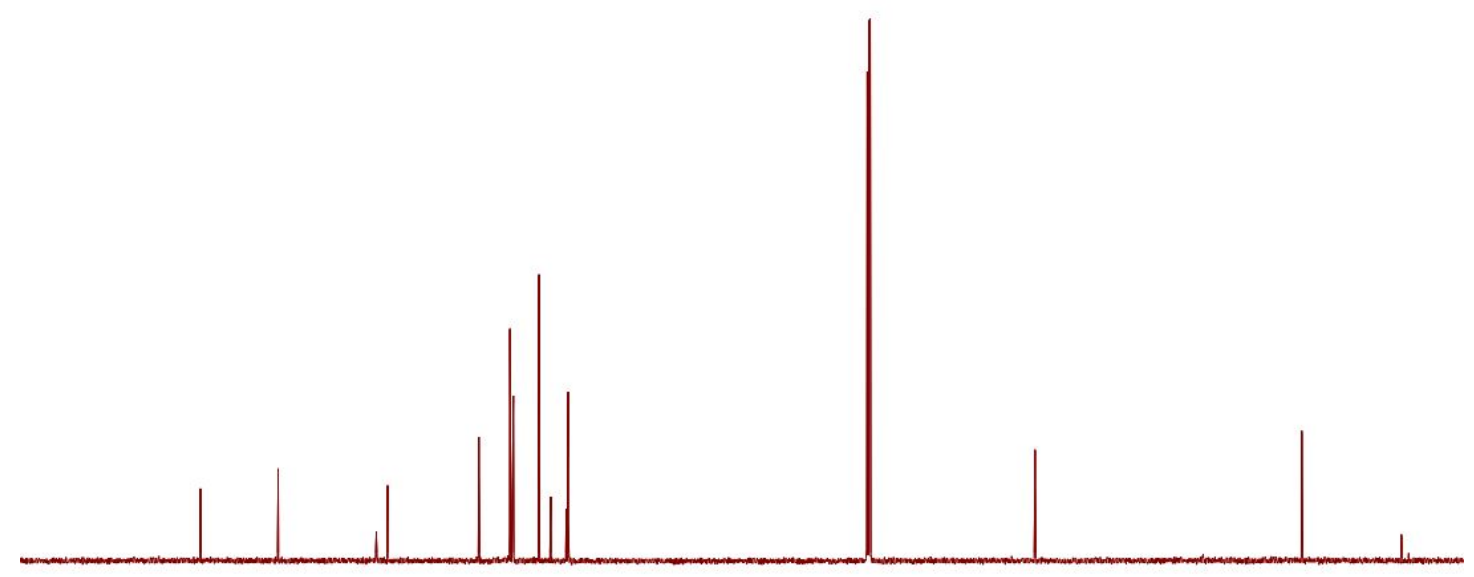

$\begin{array}{lllllllllllllllllll}190 & 180 & 170 & 160 & 150 & 140 & 130 & 120 & 110 & \underset{\mathrm{f} 1}{100}(\mathrm{ppm}) & 80 & 70 & 60 & 50 & 40 & 30 & 20 & 10 & 0\end{array}$ 
(E)-4-bromo-2-(1-((4-nitrobenzyl)imino)ethyl)phenol (2c):

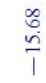

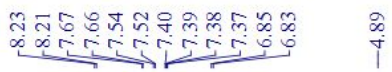<smiles>[Z20]C(=NCc1ccc([N+](=O)[O-])cc1)c1cc(Br)ccc1O</smiles>

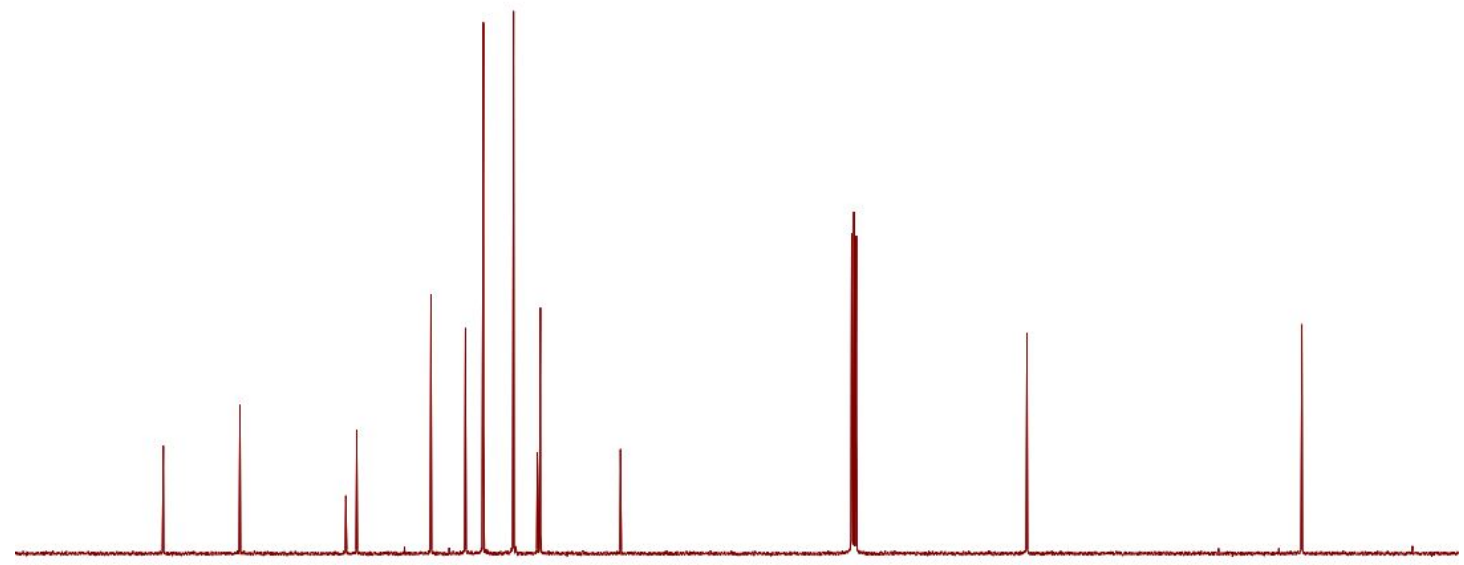

$\begin{array}{lllllllllll}190 & 180 & 170 & 160 & 150 & 140 & 130 & 120 & 110 & 100 & 90\end{array}$

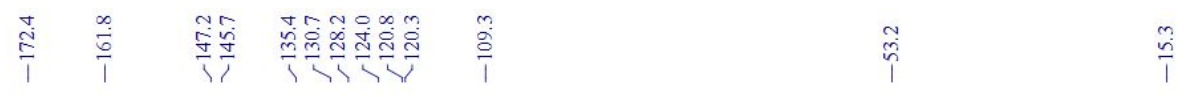


(E)-4-fluoro-2-(1-((4-nitrobenzyl)imino)ethyl)phenol (2d):

$\frac{5}{1}$

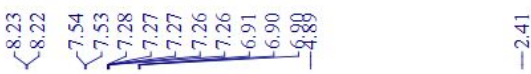<smiles>[Z10]C(=NCc1ccc([N+](=O)[O-])cc1)c1cc(F)ccc1O</smiles>

$2 d$

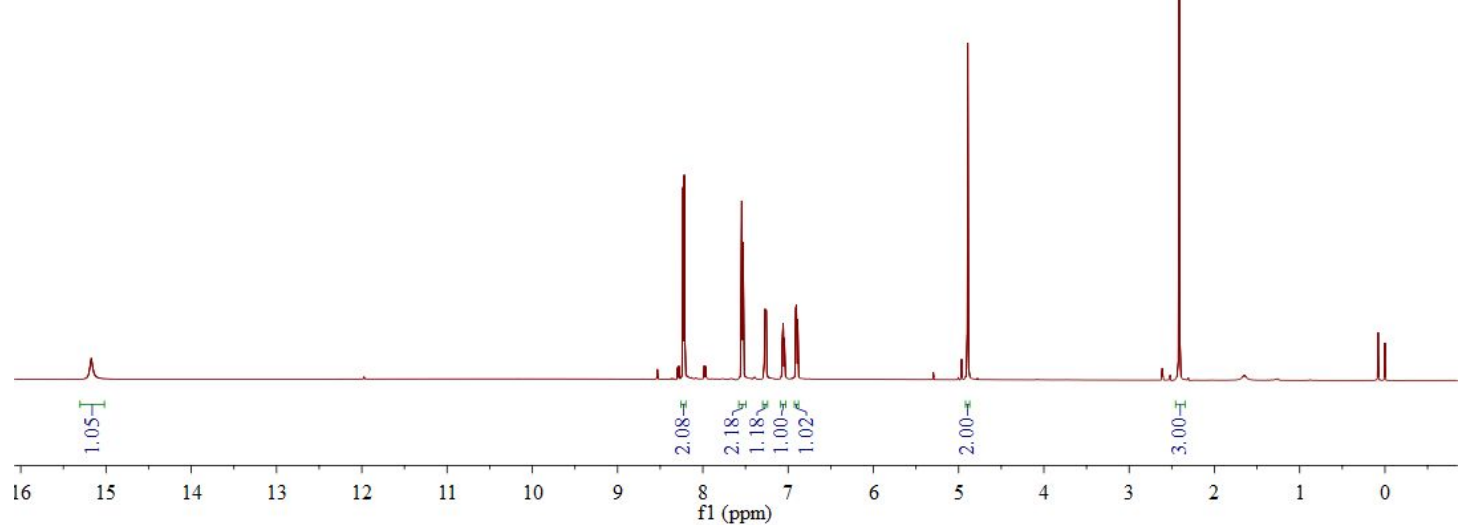

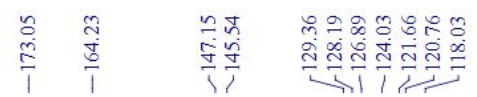

लेखू

in

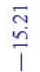

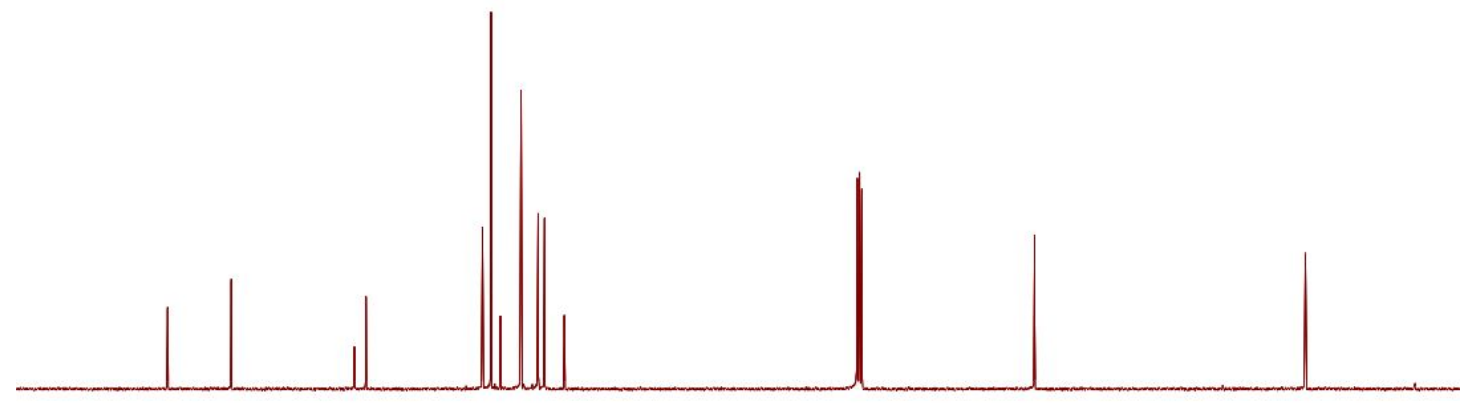

$\begin{array}{llllllllll}190 & 180 & 170 & 160 & 150 & 140 & 130 & 120 & 110 & 100 \\ \mathrm{f} 1(\mathrm{ppm})\end{array}$ 
ำ

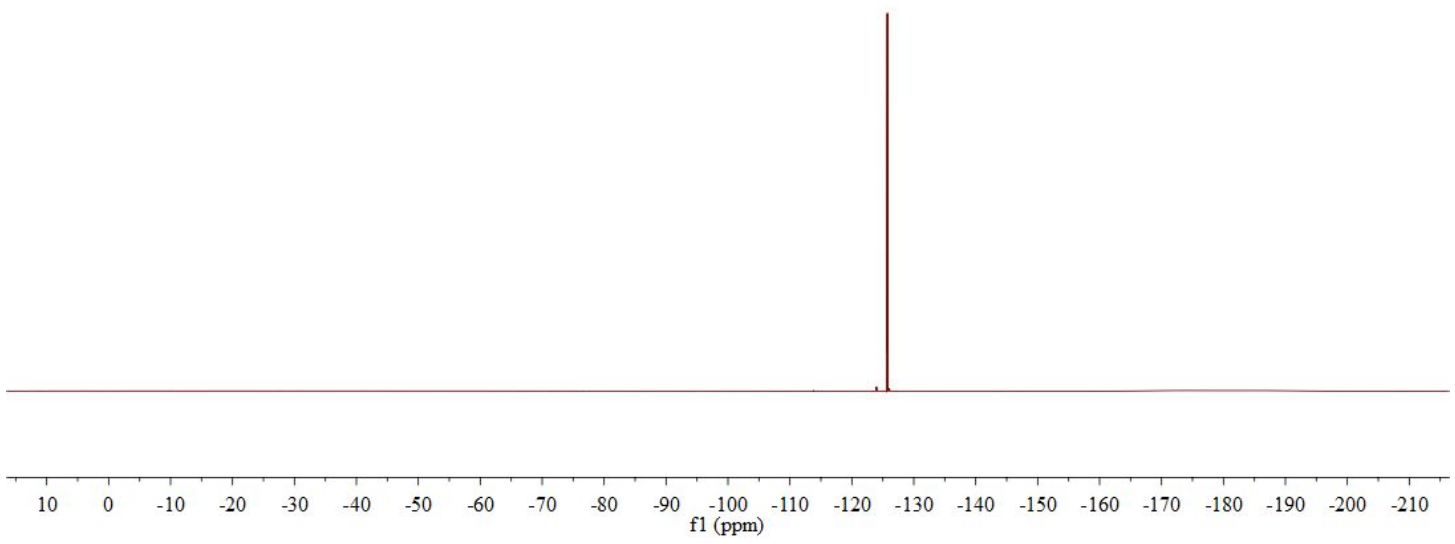

S36 
(E)-4-methyl-2-(1-((4-nitrobenzyl)imino)ethyl)phenol (2e):

$\stackrel{?}{i}$

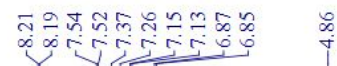

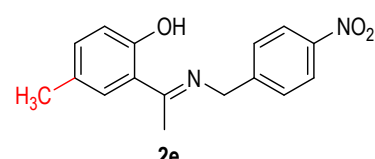

$2 e$

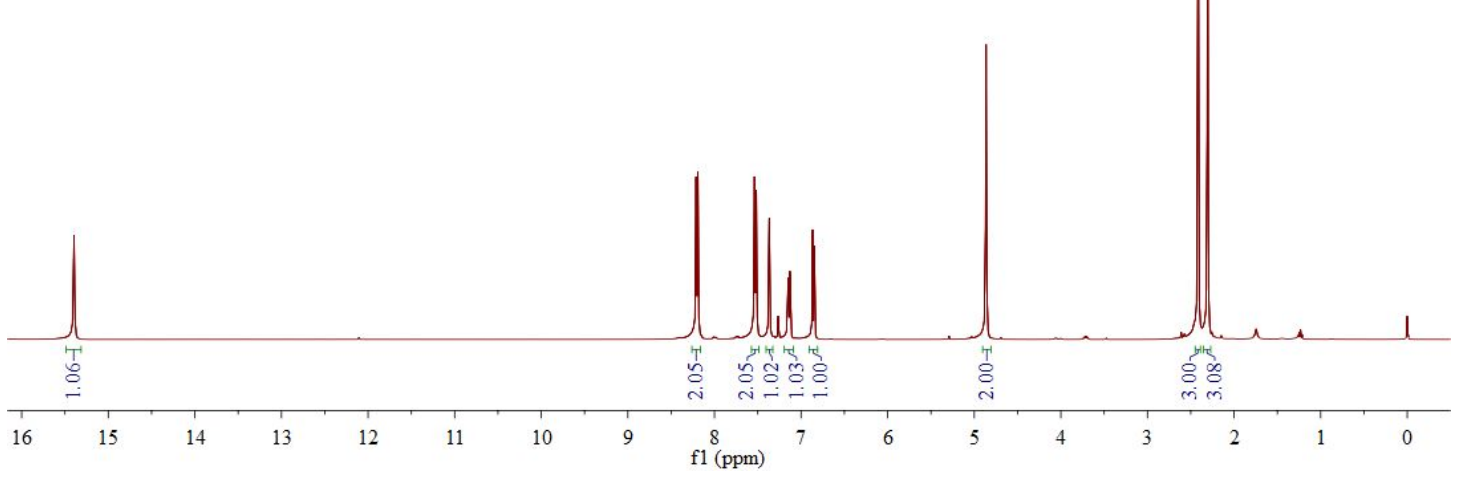

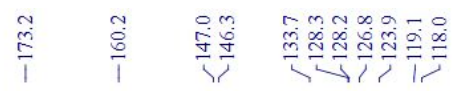

$\stackrel{\sim}{\sim} \underset{i}{\tilde{1}} \bar{i}$

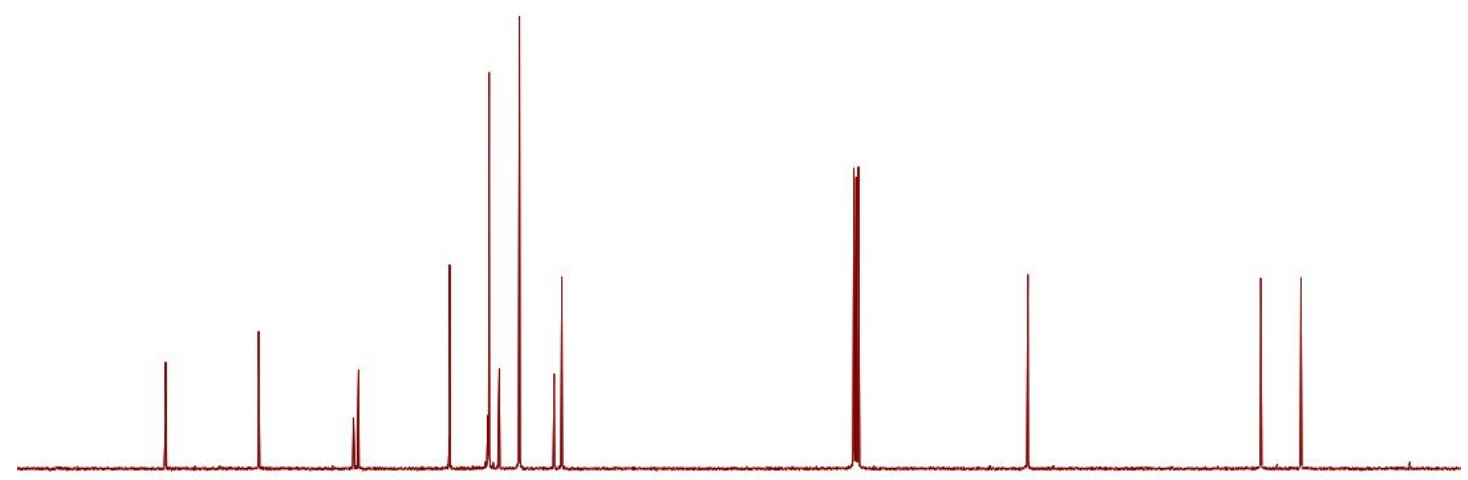

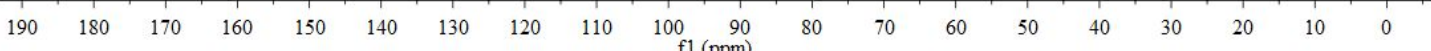


(E)-5-methoxy-2-(1-((4-nitrobenzyl)imino)ethyl)phenol (2f):

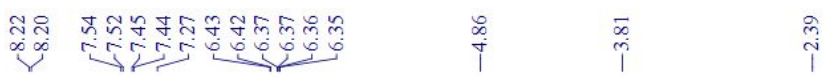<smiles>CCC(=NCc1ccc([N+](=O)[O-])cc1)c1ccc(OC)cc1O</smiles>

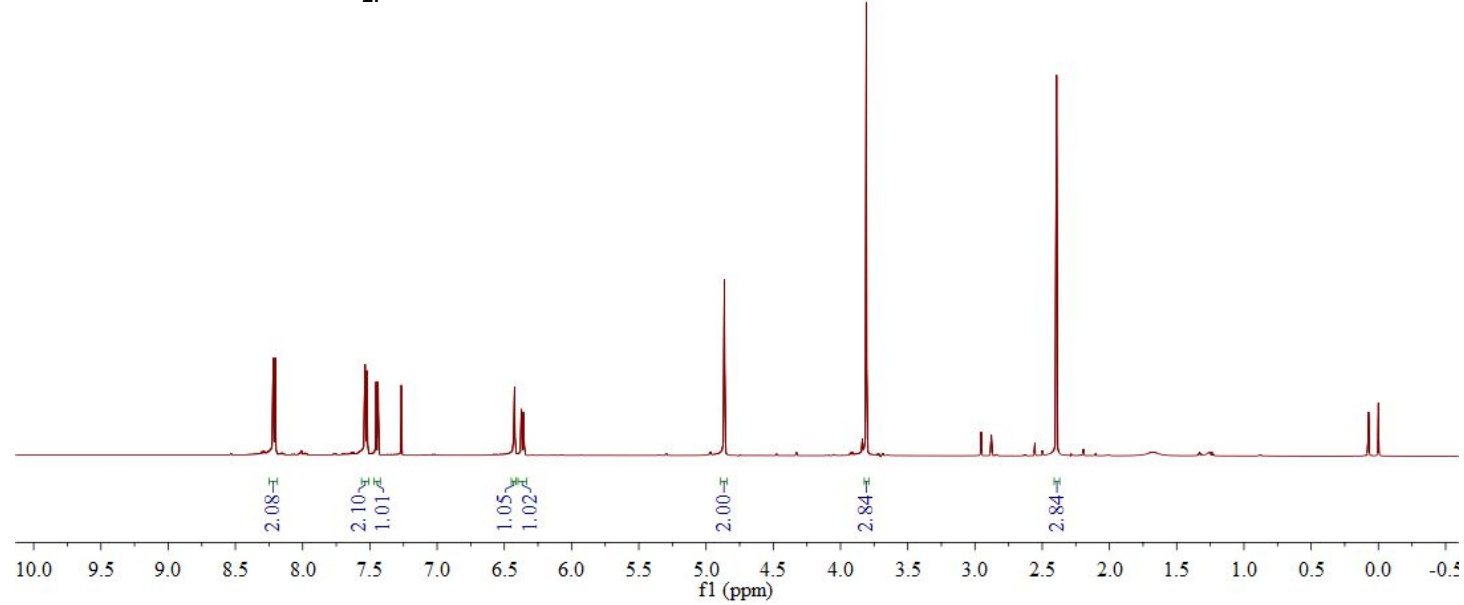

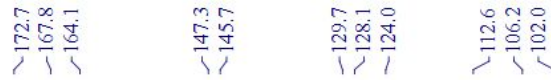

in $\quad$ i

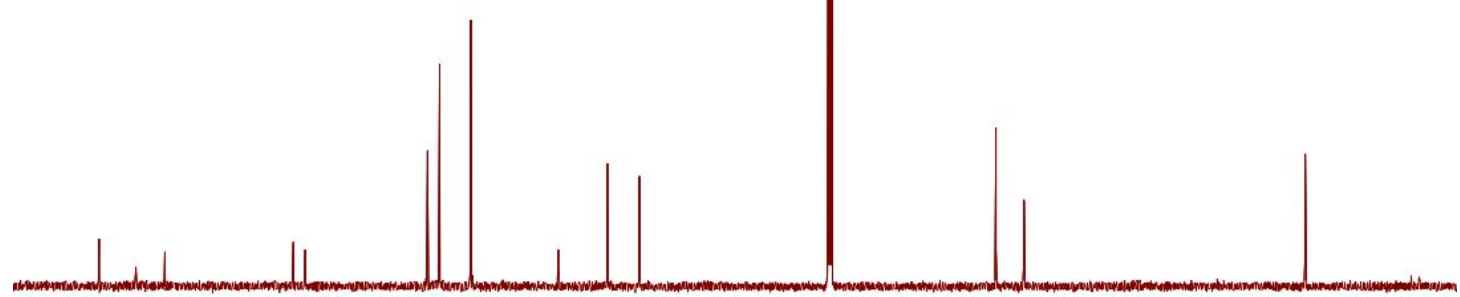

$\begin{array}{lllllllllllllllllll}180 & 170 & 160 & 150 & 140 & 130 & 120 & 110 & 100 & 90 & 80 & 70 & 60 & 50 & 40 & 30 & 20 & 10 & 0\end{array}$ 
(E)-5-chloro-2-(1-((4-nitrobenzyl)imino)ethyl)phenol (2g):

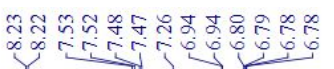

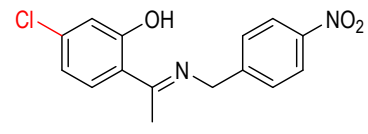

$2 g$

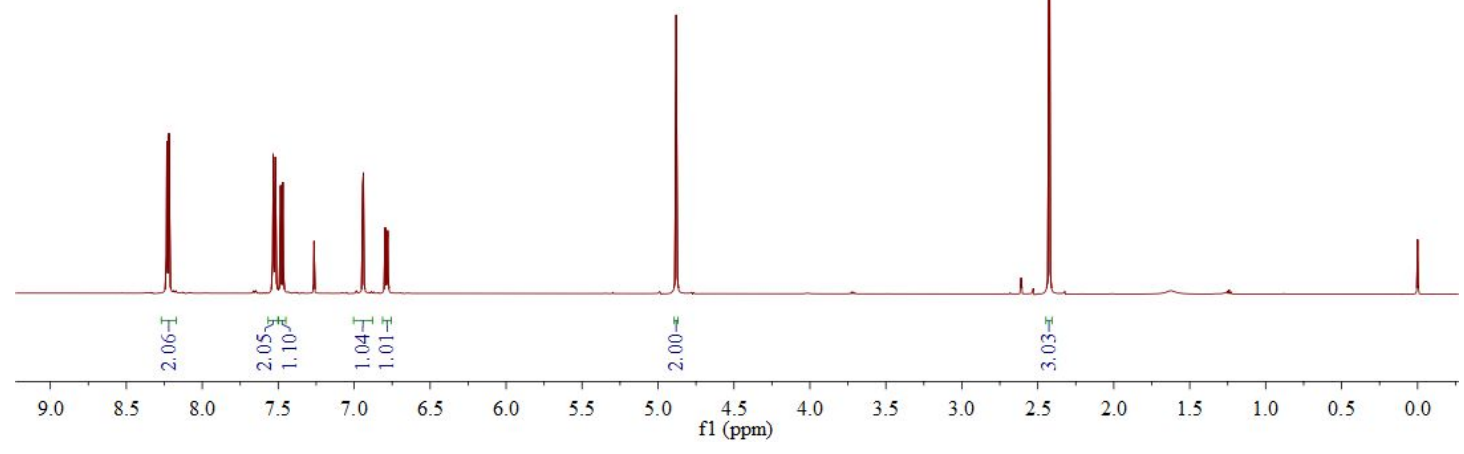

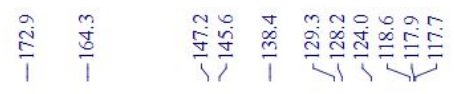

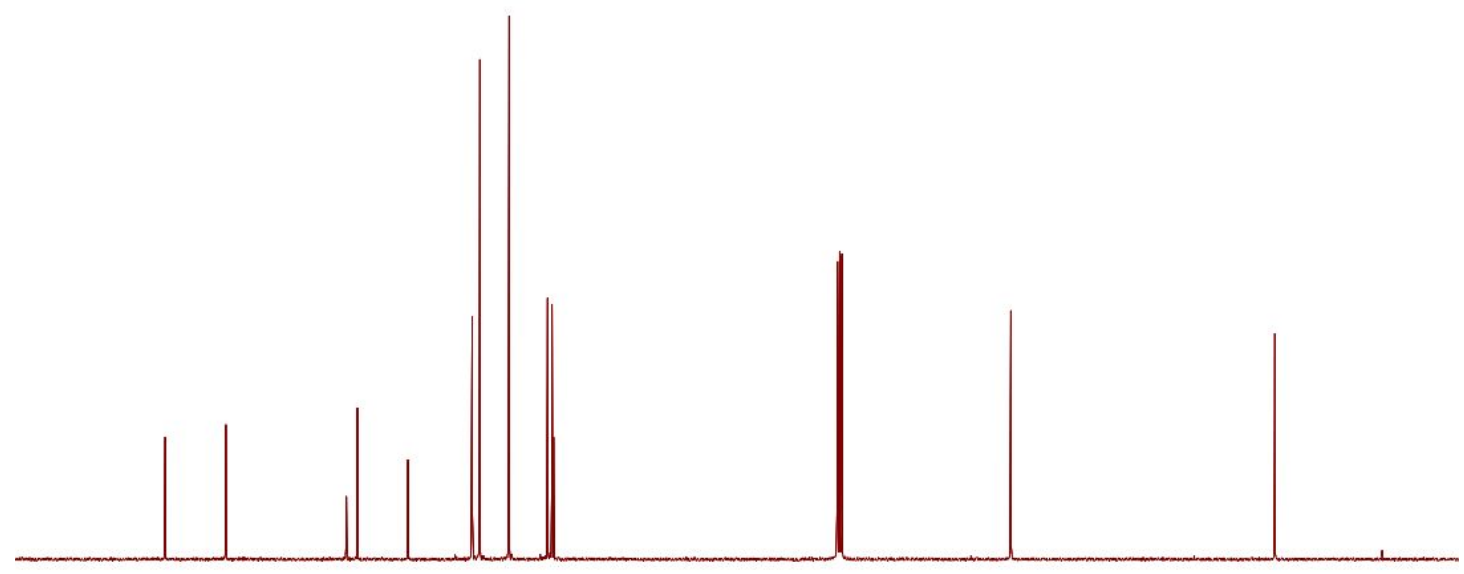

$\begin{array}{llllllllllllllllllllll}190 & 180 & 170 & 160 & 150 & 140 & 130 & 120 & 110 & 100 & \begin{array}{c}10 \\ \mathrm{f} 1(\mathrm{pm})\end{array} & 80 & 70 & 60 & 50 & 40 & 30 & 20 & 10 & 0 & -11\end{array}$ 
(E)-5-bromo-2-(1-((4-nitrobenzyl)imino)ethyl)phenol (2h):

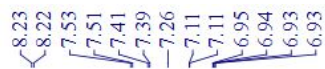<smiles>CC(=NCc1ccc([N+](=O)[O-])cc1)c1ccc(Br)cc1O</smiles>

2h

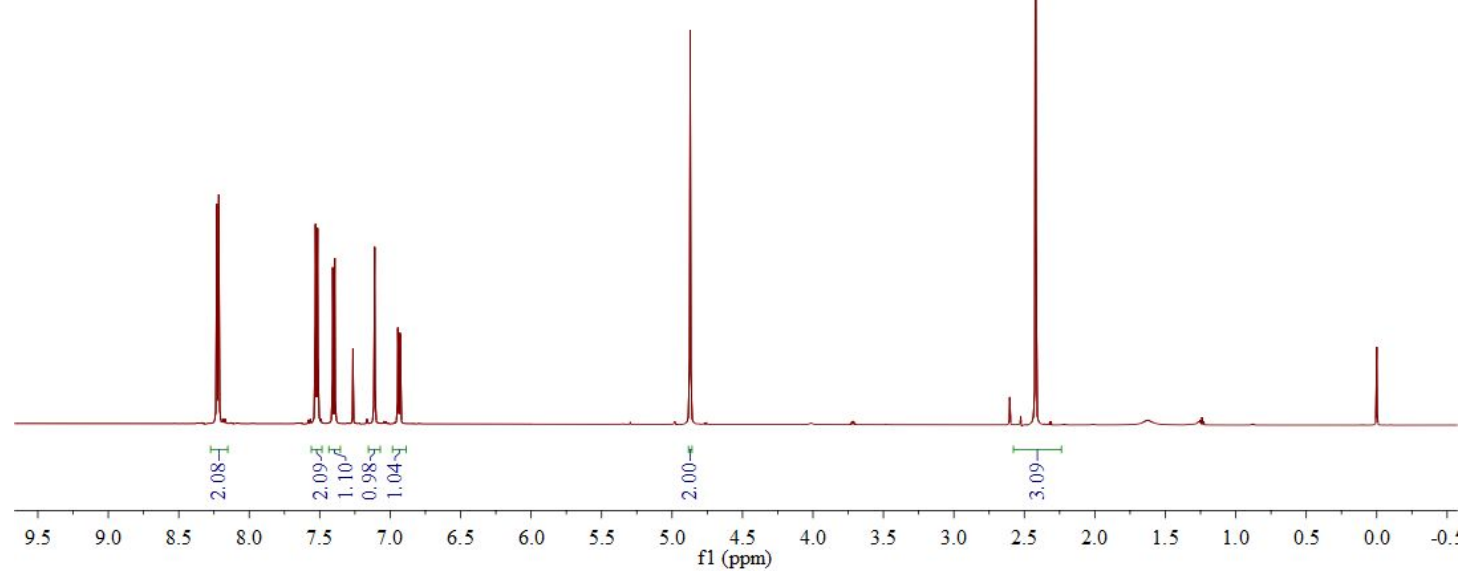

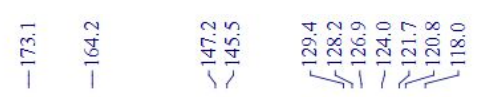
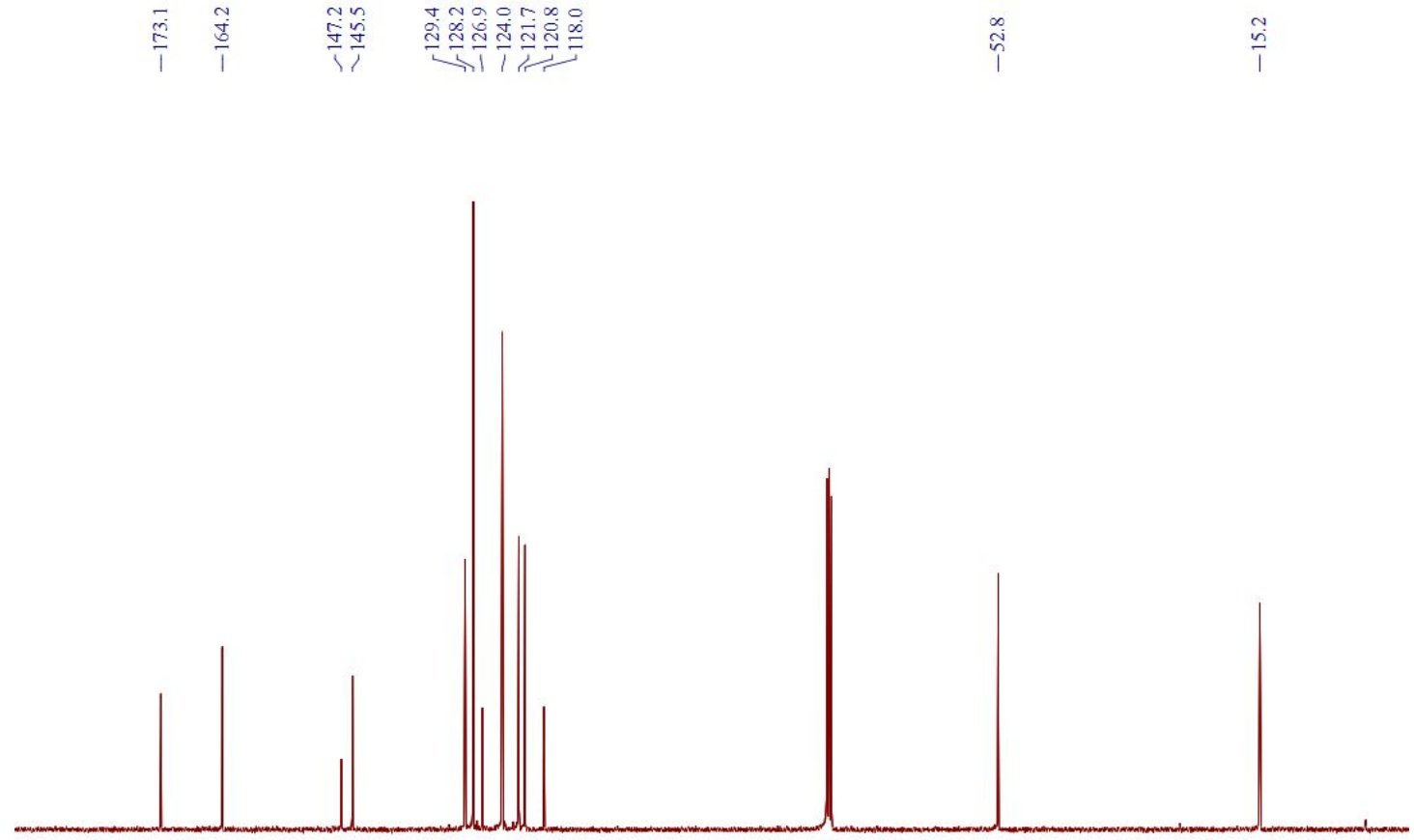

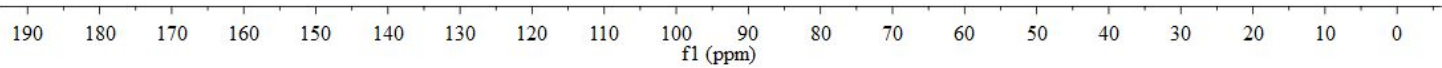


(E)-5-methyl-2-(1-((4-nitrobenzyl)imino)ethyl)phenol (2i):
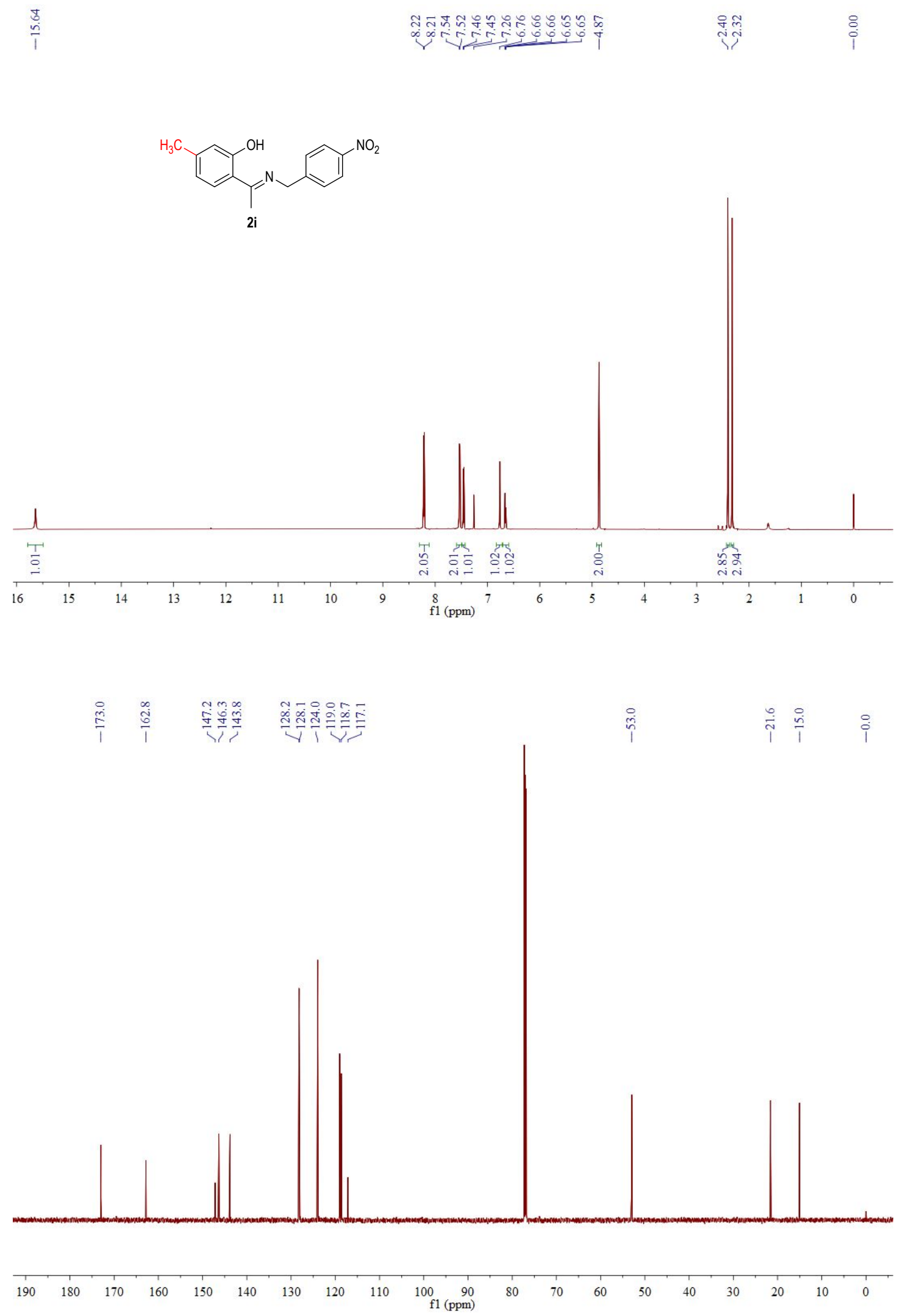
(E)-4-nitro-2-(1-((4-nitrobenzyl)imino)ethyl)phenol (2j):

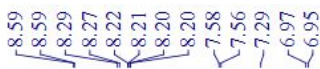

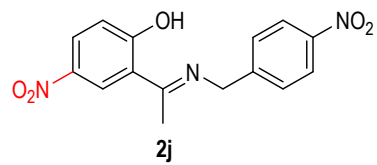

2j
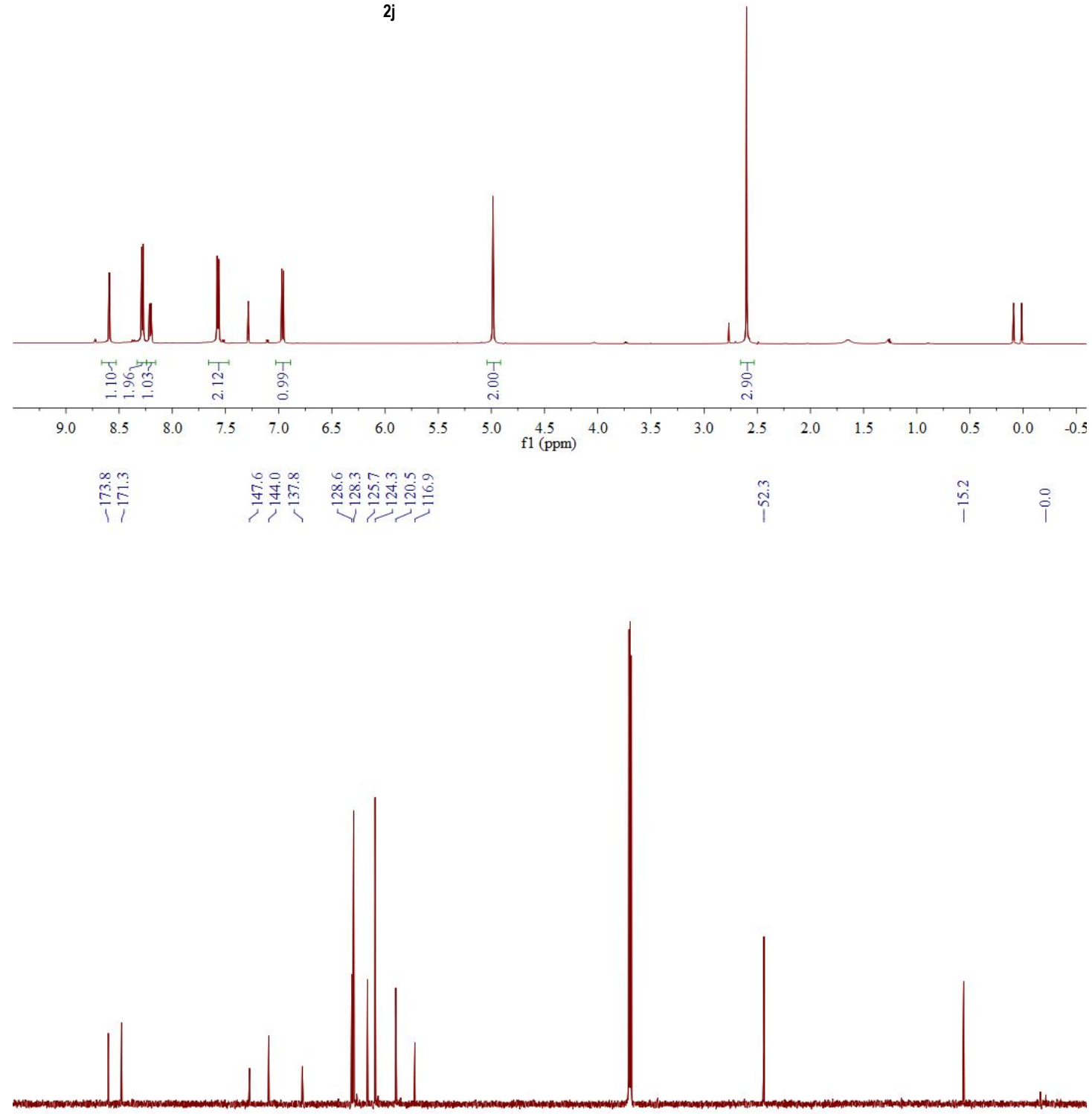

190 180 160 150 $100 \underset{f 1(\mathrm{ppm})}{90}$ 
(E)-3-methoxy-2-(1-((4-nitrobenzyl)imino)ethyl)phenol (2k):

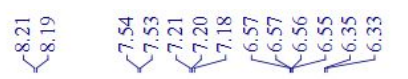
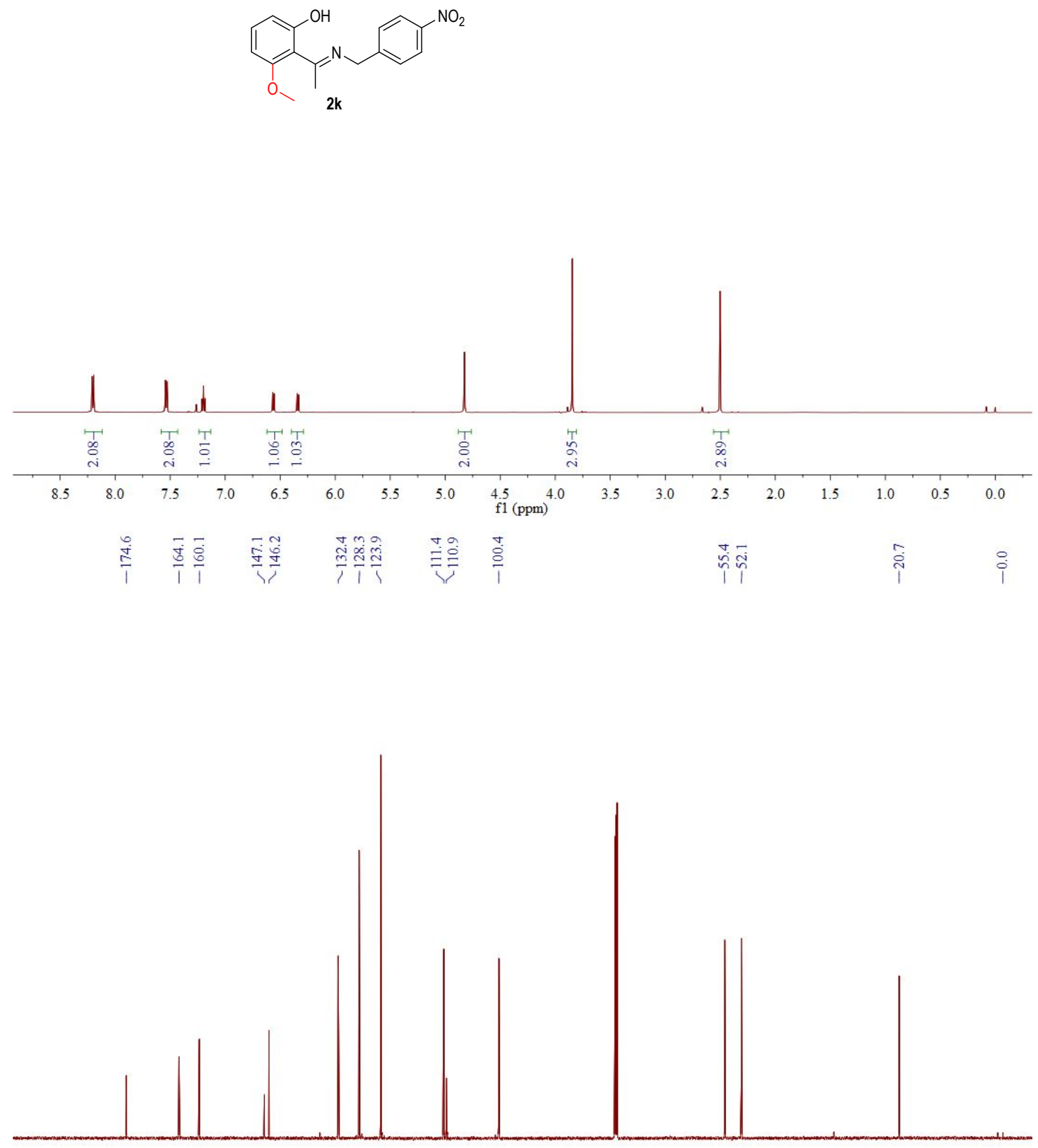

$\begin{array}{lllllllllllllllllll}190 & 180 & 170 & 160 & 150 & 140 & 130 & 120 & 110 & \underset{\mathrm{f} 1(\mathrm{ppm})}{100} & 80 & 70 & 60 & 50 & 40 & 30 & 20 & 10 & 0\end{array}$ 
(E)-2-(1-((4-nitrobenzyl)imino)propyl)phenol (21):
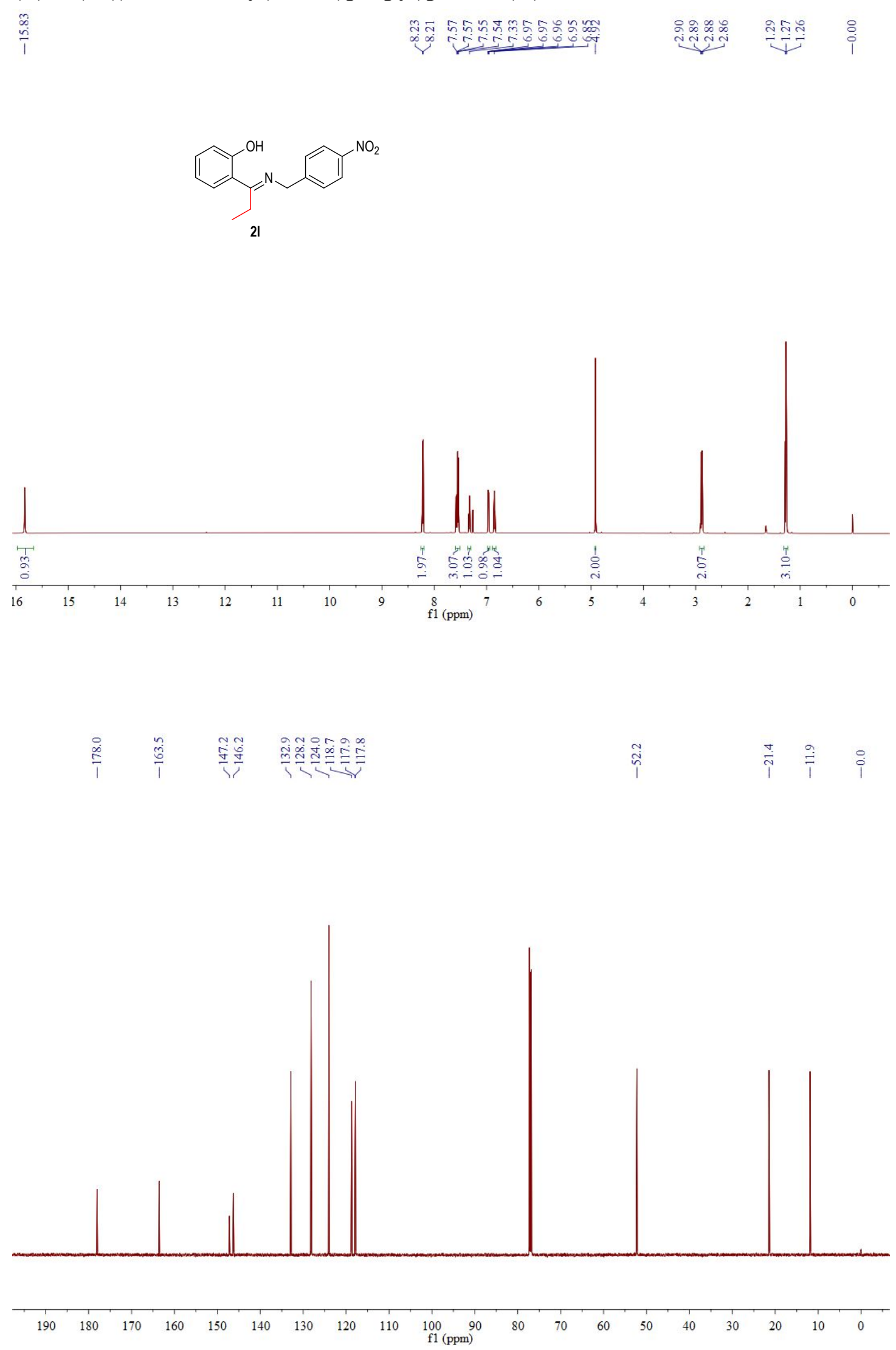
(E)-2-(((4-nitrobenzyl)imino)(phenyl)methyl)phenol (2m):

ind<smiles>O=[N+]([O-])c1ccc(CN=C(c2ccccc2)c2ccccc2O)cc1</smiles>
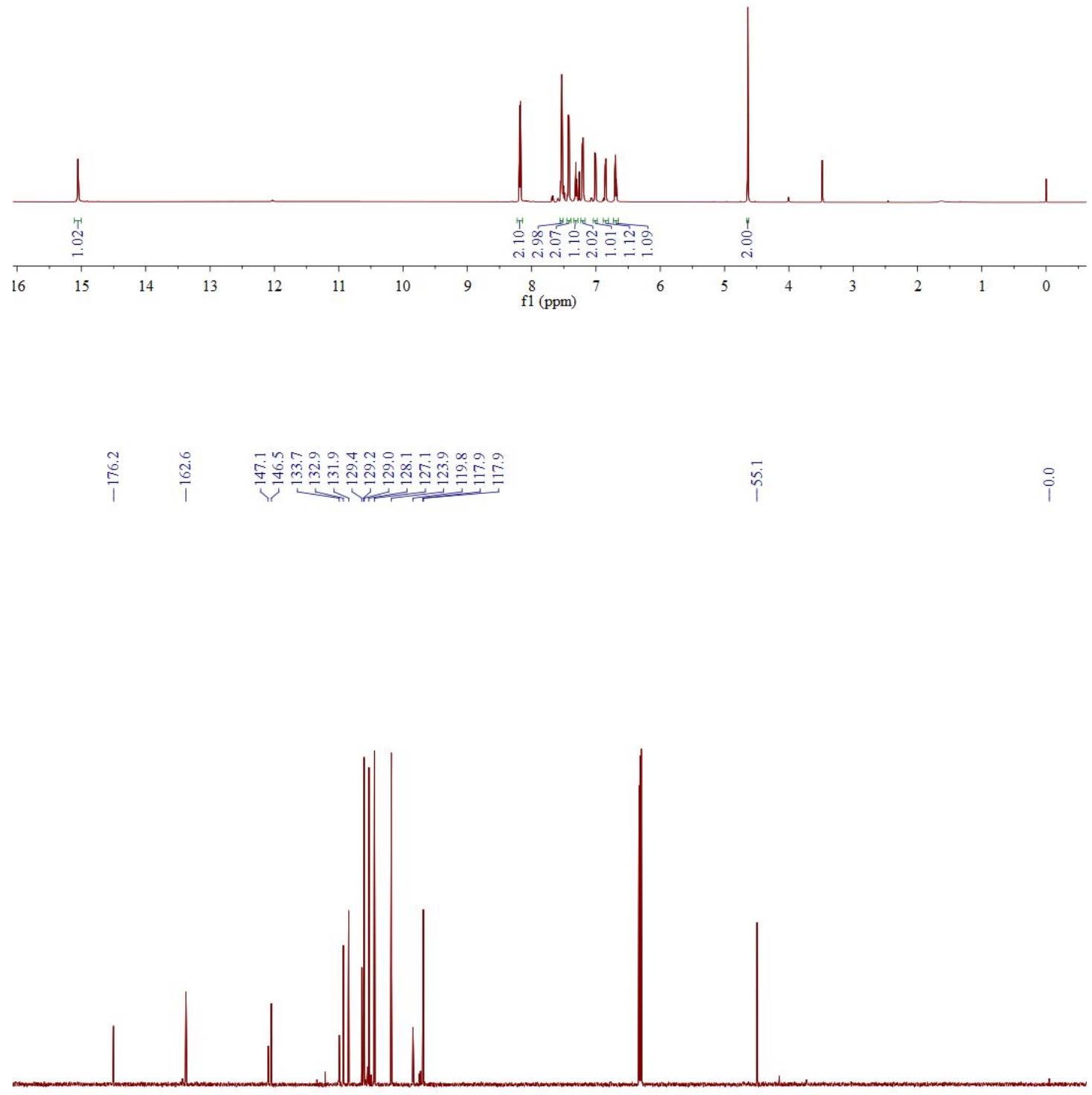

$\begin{array}{lllllllllllllllllll}190 & 180 & 170 & 160 & 150 & 140 & 130 & 120 & 110 & \underset{\mathrm{f} 1(\mathrm{ppm})}{100} & 80 & 70 & 60 & 50 & 40 & 30 & 20 & 10 & 0\end{array}$ 
(E)-2-(1-((2-nitrobenzyl)imino)ethyl)phenol (2aa):

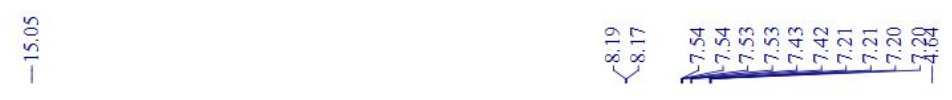

$\stackrel{\infty}{i}$

$\stackrel{8}{i}$
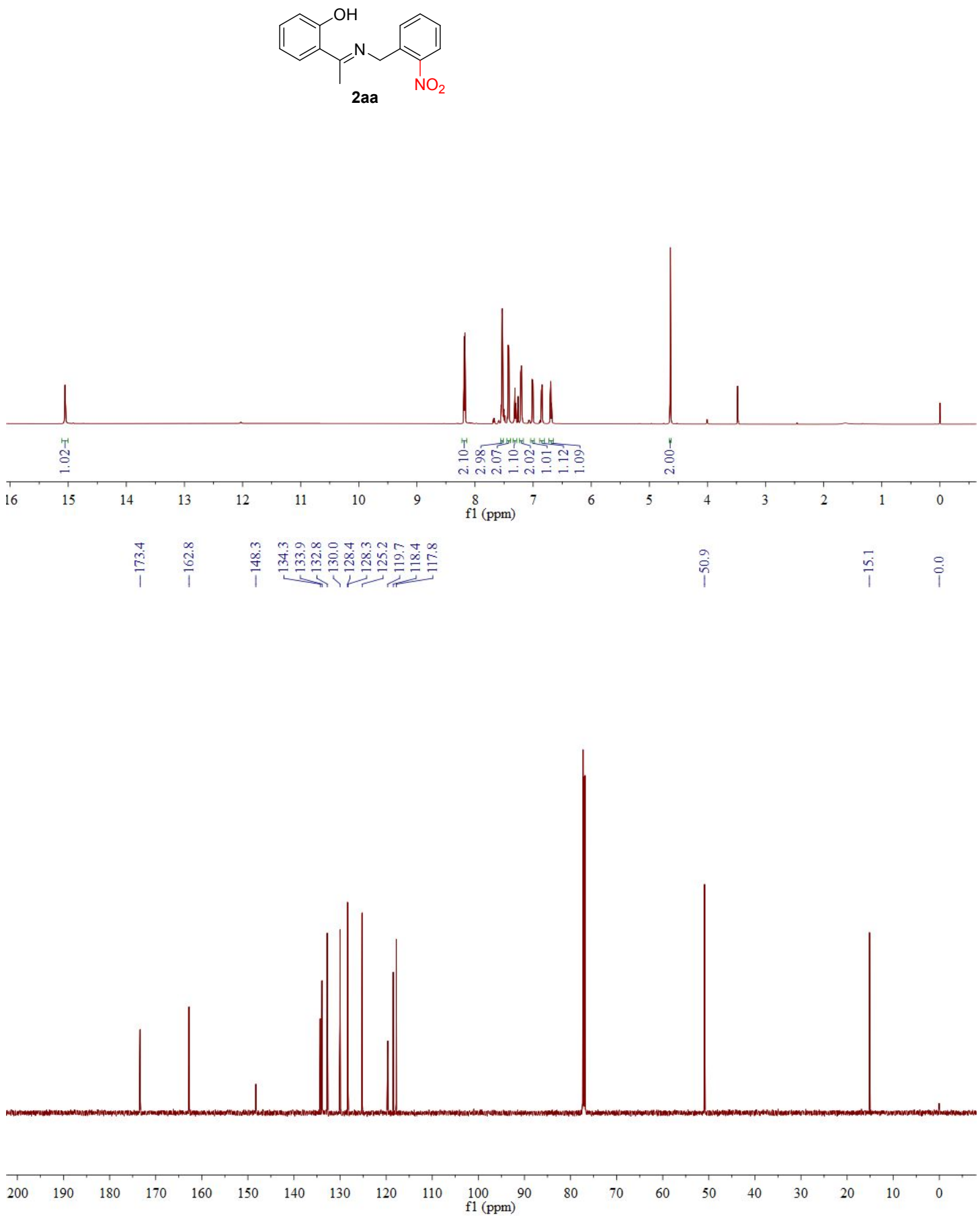

S46 
(E)-2-(1-((3-nitrobenzyl)imino)ethyl)phenol (2ab):

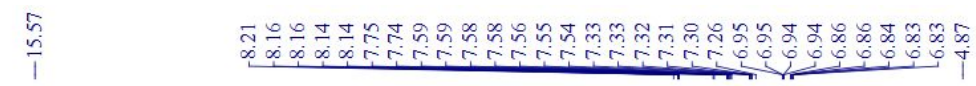

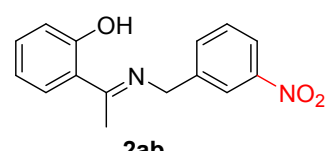

2ab

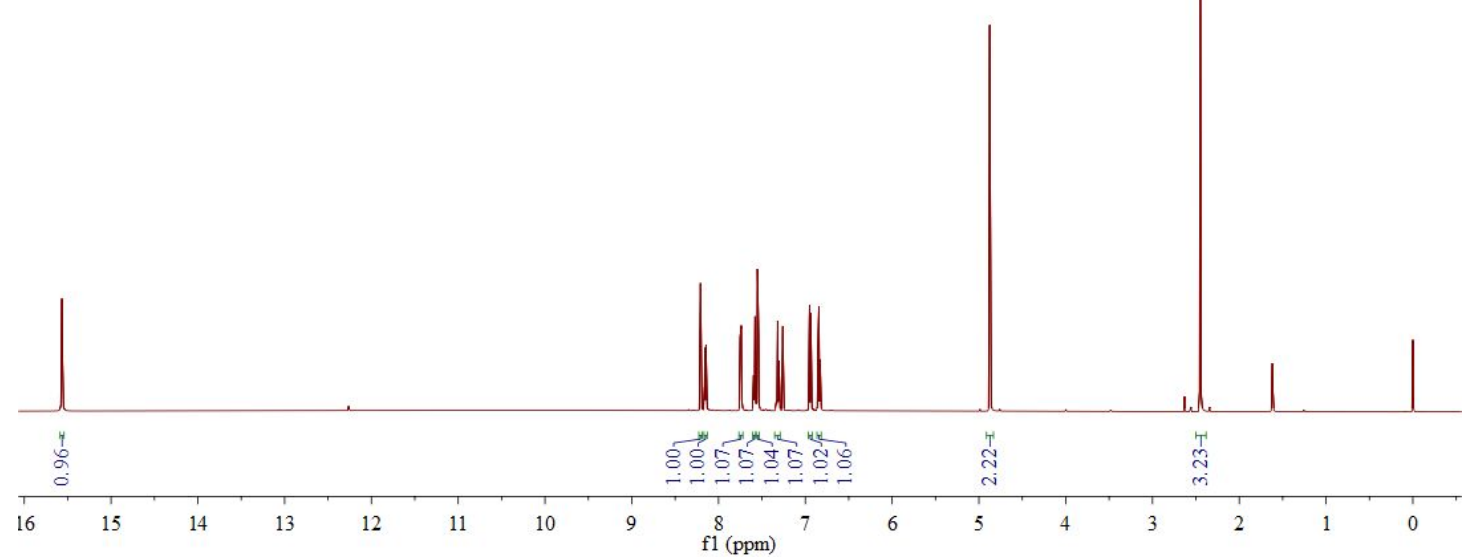

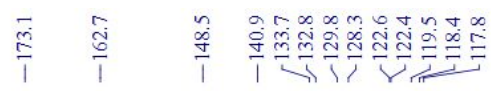

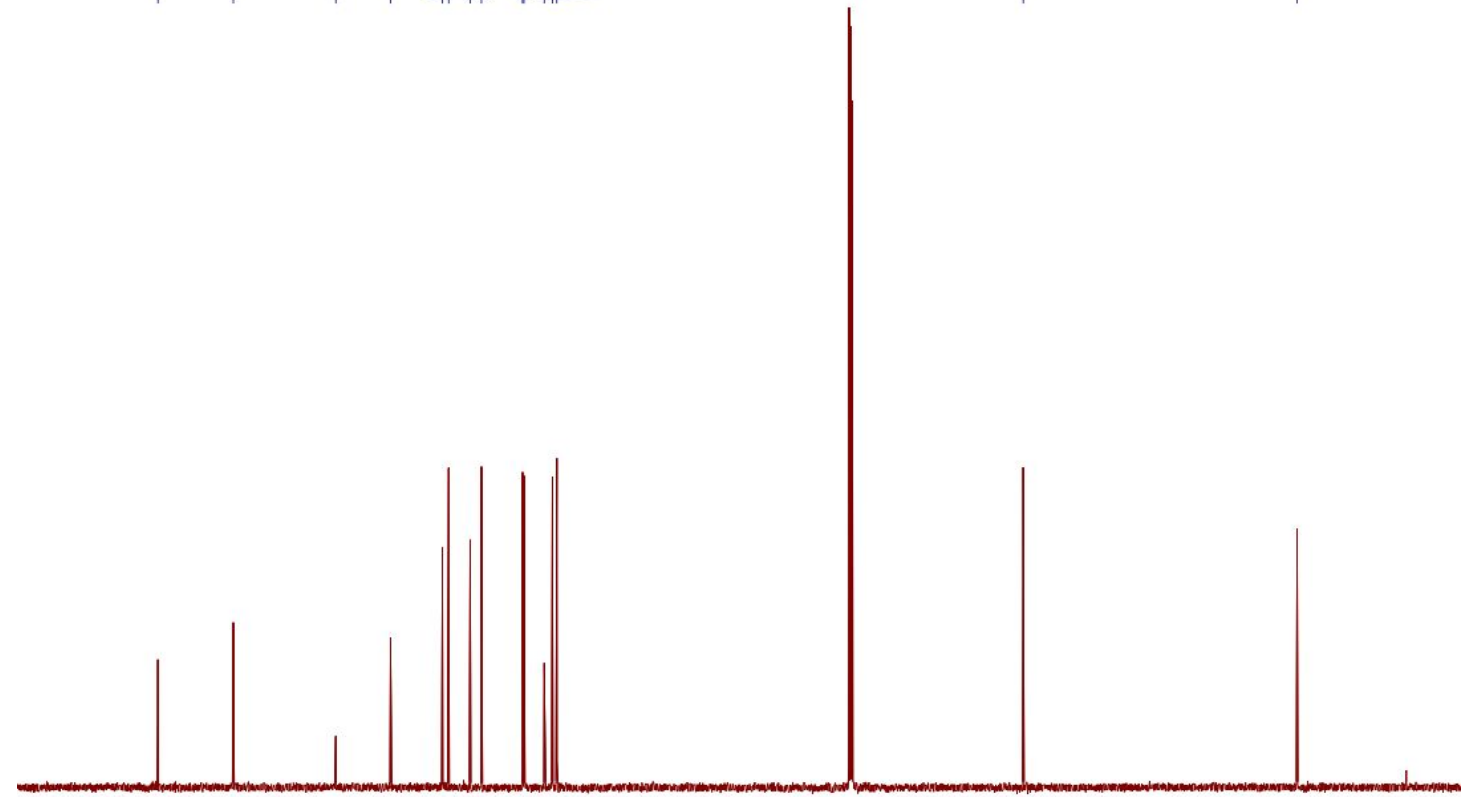

190

$\begin{array}{lllllllll}180 & 170 & 160 & 150 & 140 & 130 & 120 & 110 & 100 \begin{array}{r}90 \\ \mathrm{f} 1(\mathrm{ppm})\end{array}\end{array}$

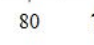

60

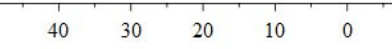


(E)-2-(1-((3,5-bis(trifluoromethyl)benzyl)imino)ethyl)phenol (2ac):

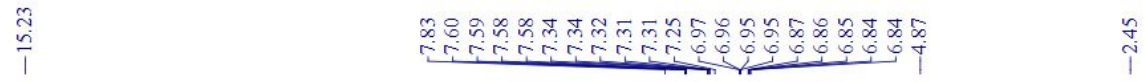
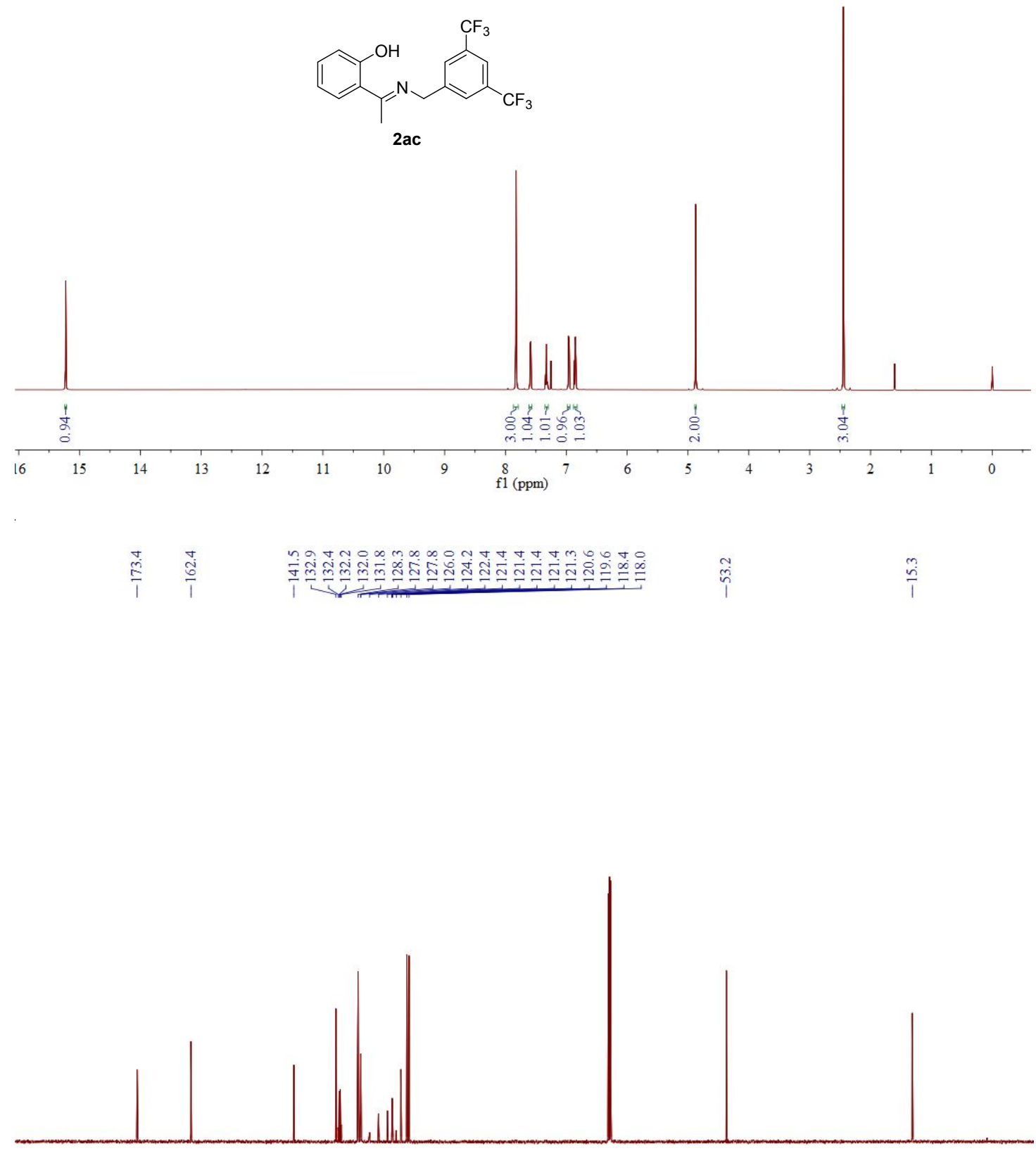

$\begin{array}{lllllllllllllllllll}190 & 180 & 170 & 160 & 150 & 140 & 130 & 120 & 110 & \underset{\mathrm{f} 1(\mathrm{ppm})}{100} & 80 & 70 & 60 & 50 & 40 & 30 & 20 & 10 & 0\end{array}$ 


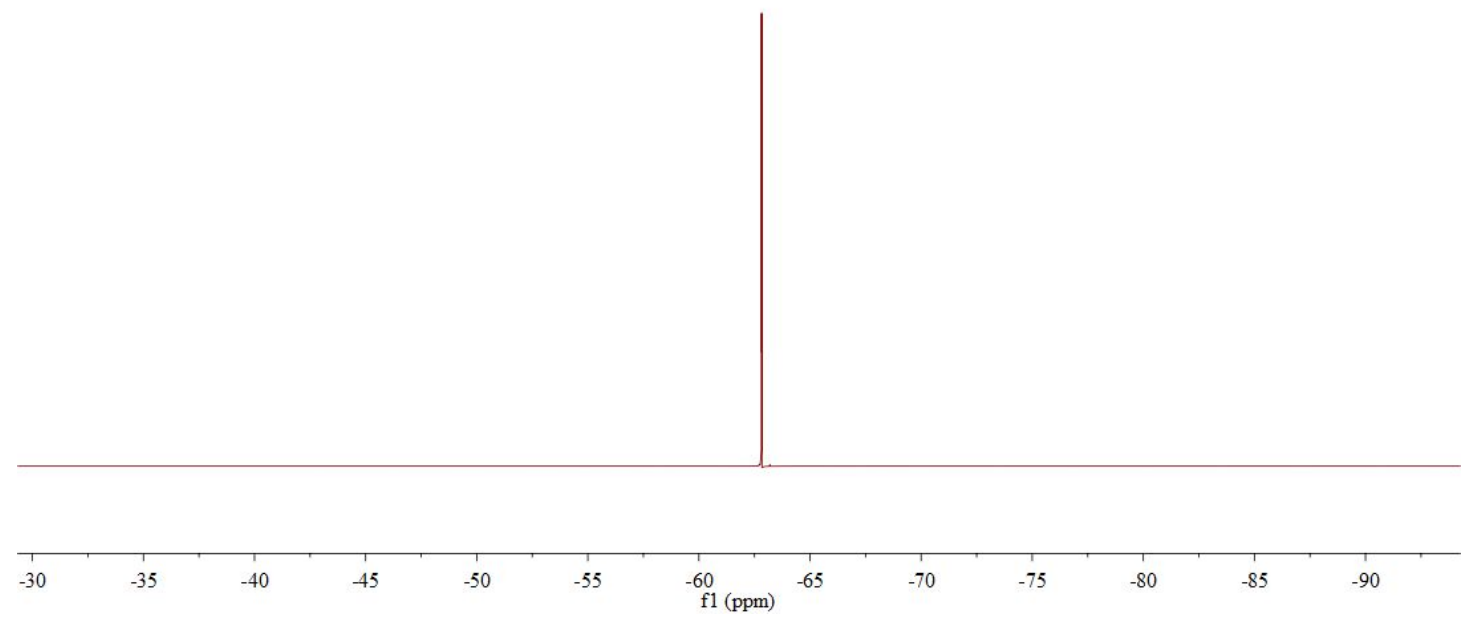


(E)-4-(((1-(2-hydroxyphenyl)ethylidene)amino)methyl)benzonitrile (2ad):
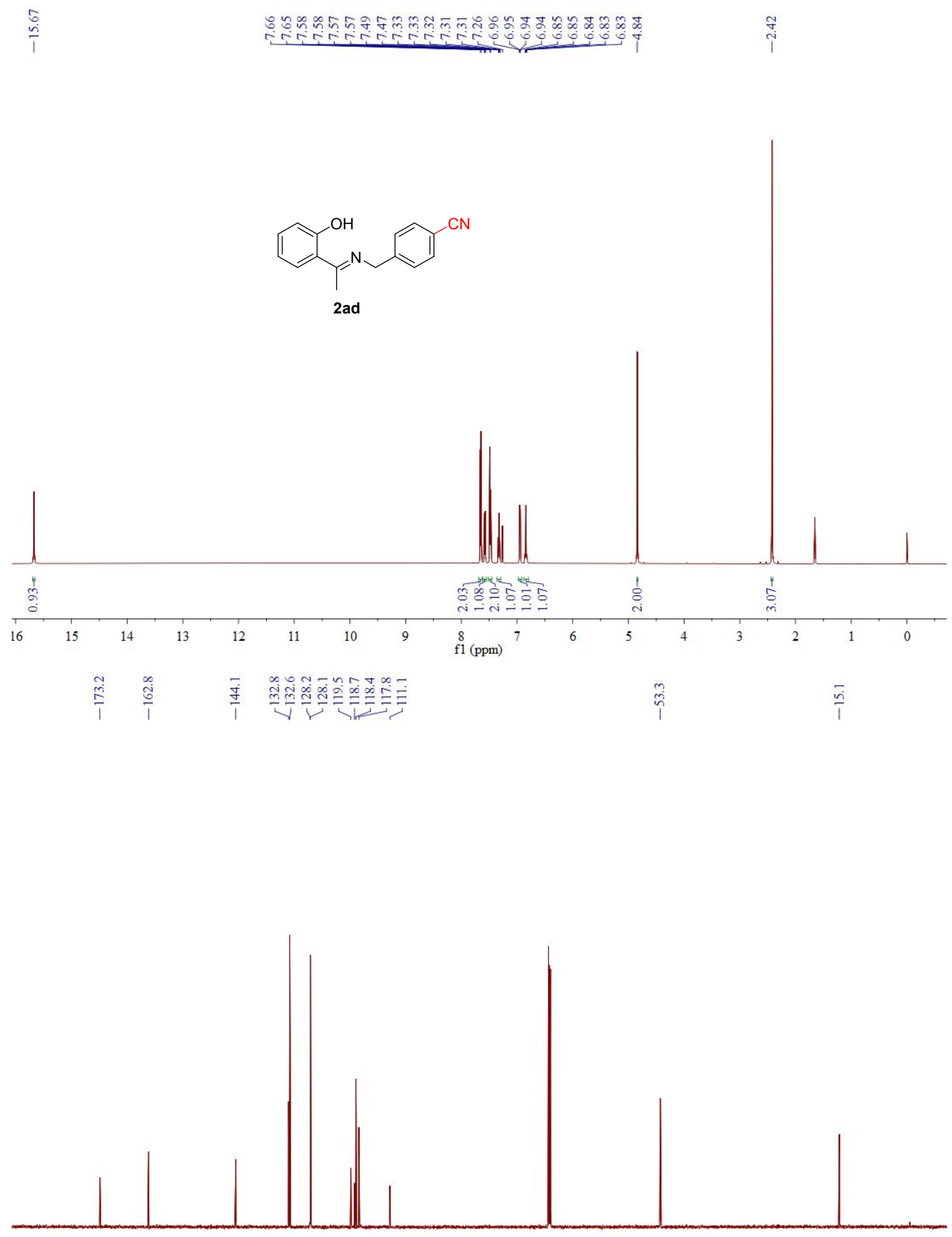

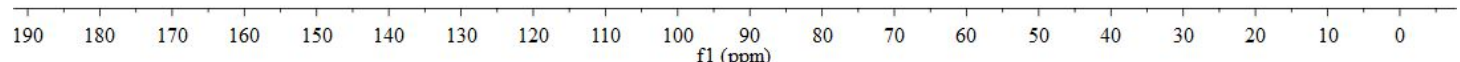


(2R,3S,3aR,9bS)-9b-methyl-2-(4-nitrophenyl)-3-phenyl-2,3,3a,9b-tetrahydrochro meno[4,3-b]pyrrol-4(1H)-one (3a):

nूक
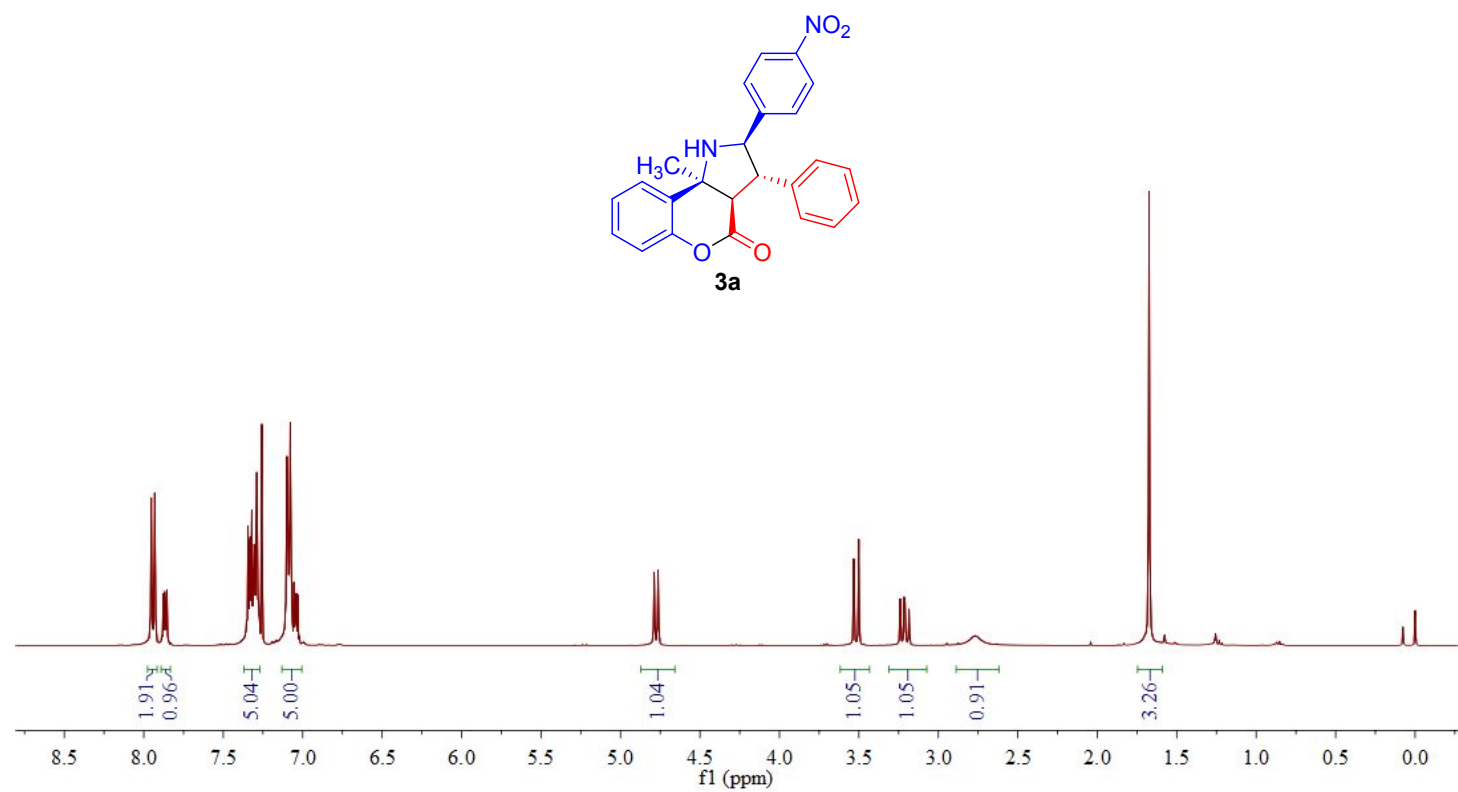

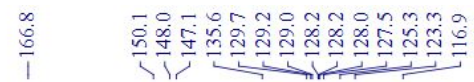

i

ஸे

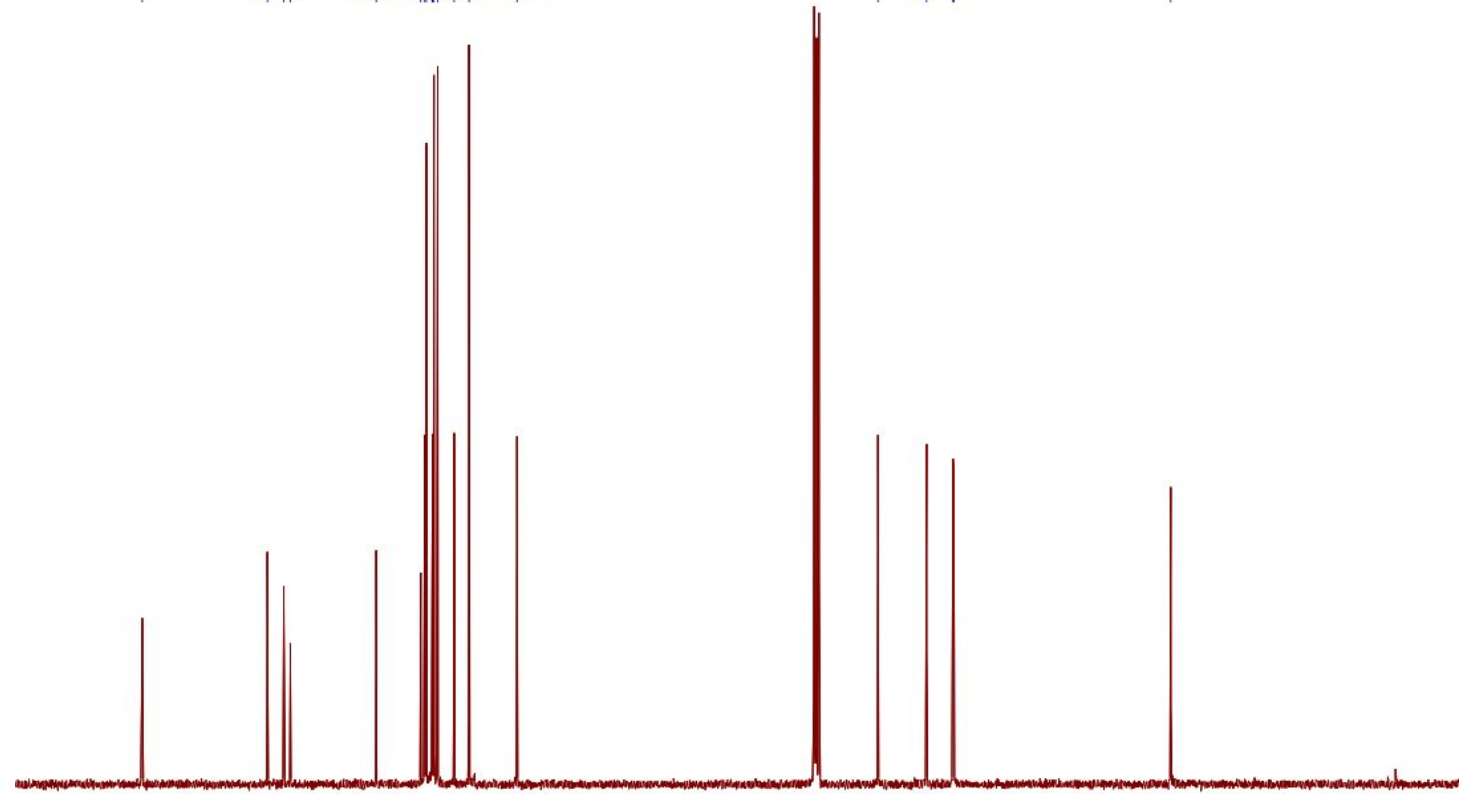

$\begin{array}{lllllllllll}180 & 170 & 160 & 150 & 140 & 130 & 120 & 110 & 100 & 90 & 80\end{array}$

$10 \quad 0$ 


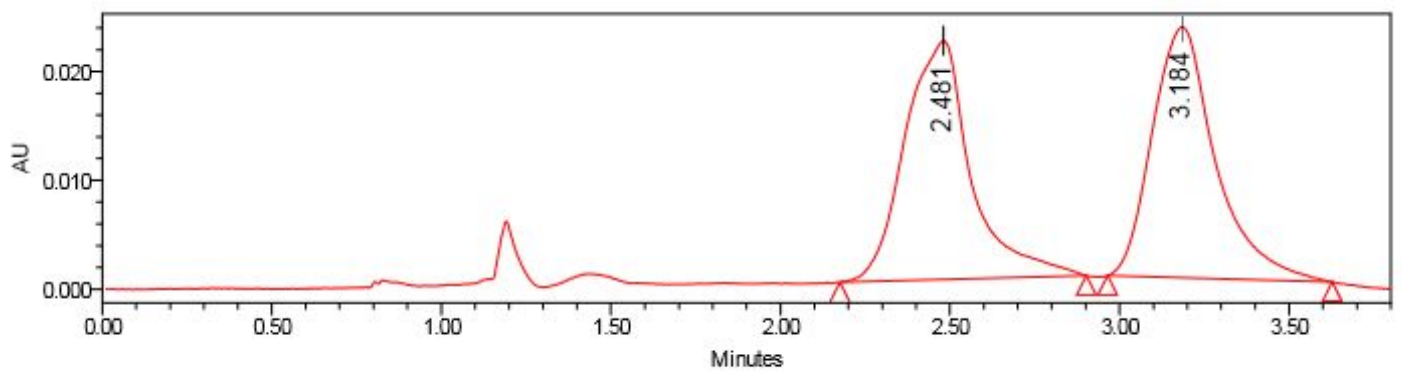

Peak Results

\begin{tabular}{|c|c|c|r|r|}
\hline & RT & Area & Height & $\%$ Area \\
\hline 1 & 2.481 & 298062 & 21931 & 49.96 \\
\hline 2 & 3.184 & 298500 & 23010 & 50.04 \\
\hline
\end{tabular}

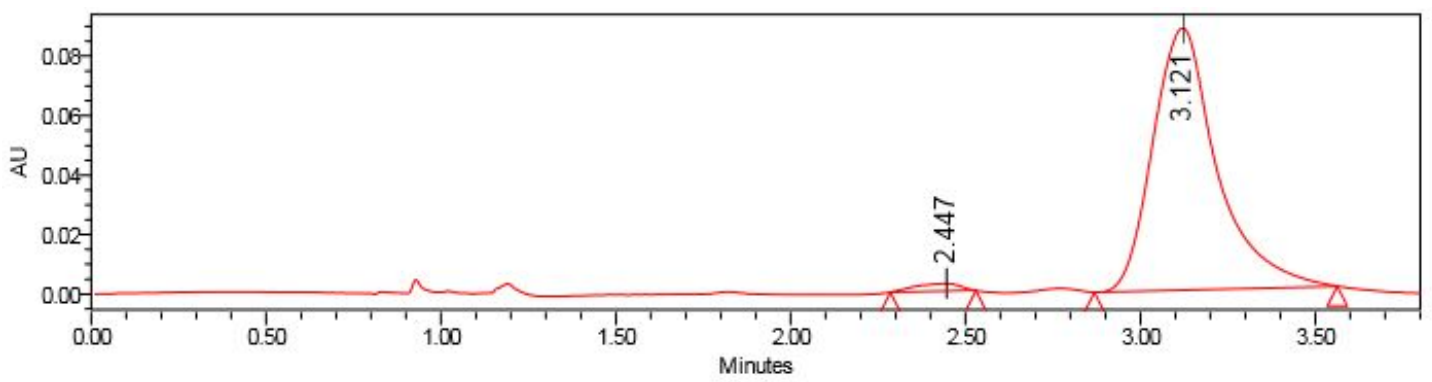

Peak Results

\begin{tabular}{|r|c|r|r|r|}
\hline & RT & Area & Height & $\%$ Area \\
\hline 1 & 2.447 & 22586 & 2527 & 1.97 \\
\hline 2 & 3.121 & 1123209 & 88132 & 98.03 \\
\hline
\end{tabular}


(2R,3S,3aR,9bS)-3-(2-fluorophenyl)-9b-methyl-2-(4-nitrophenyl)-2,3,3a,9b-tetrah ydrochromeno[4,3-b]pyrrol-4(1H)-one $(3 \mathrm{~b})$ :

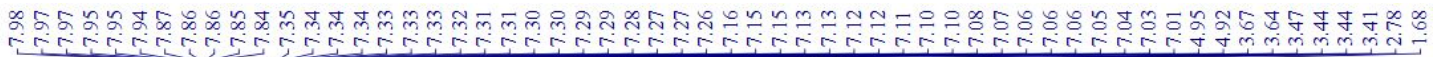

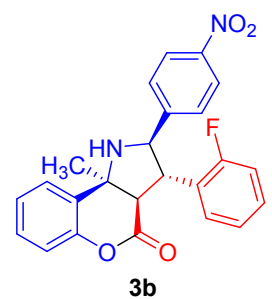

$3 b$

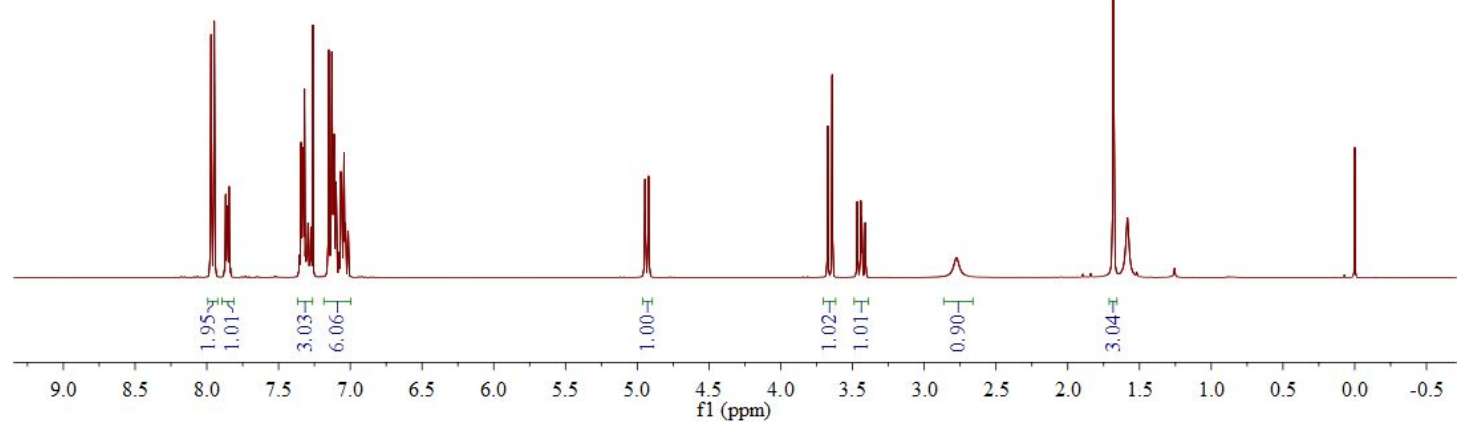

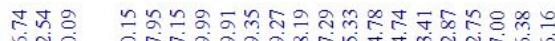

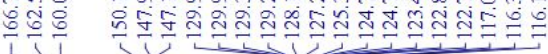

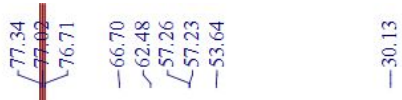

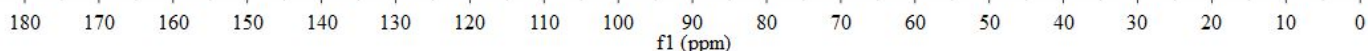



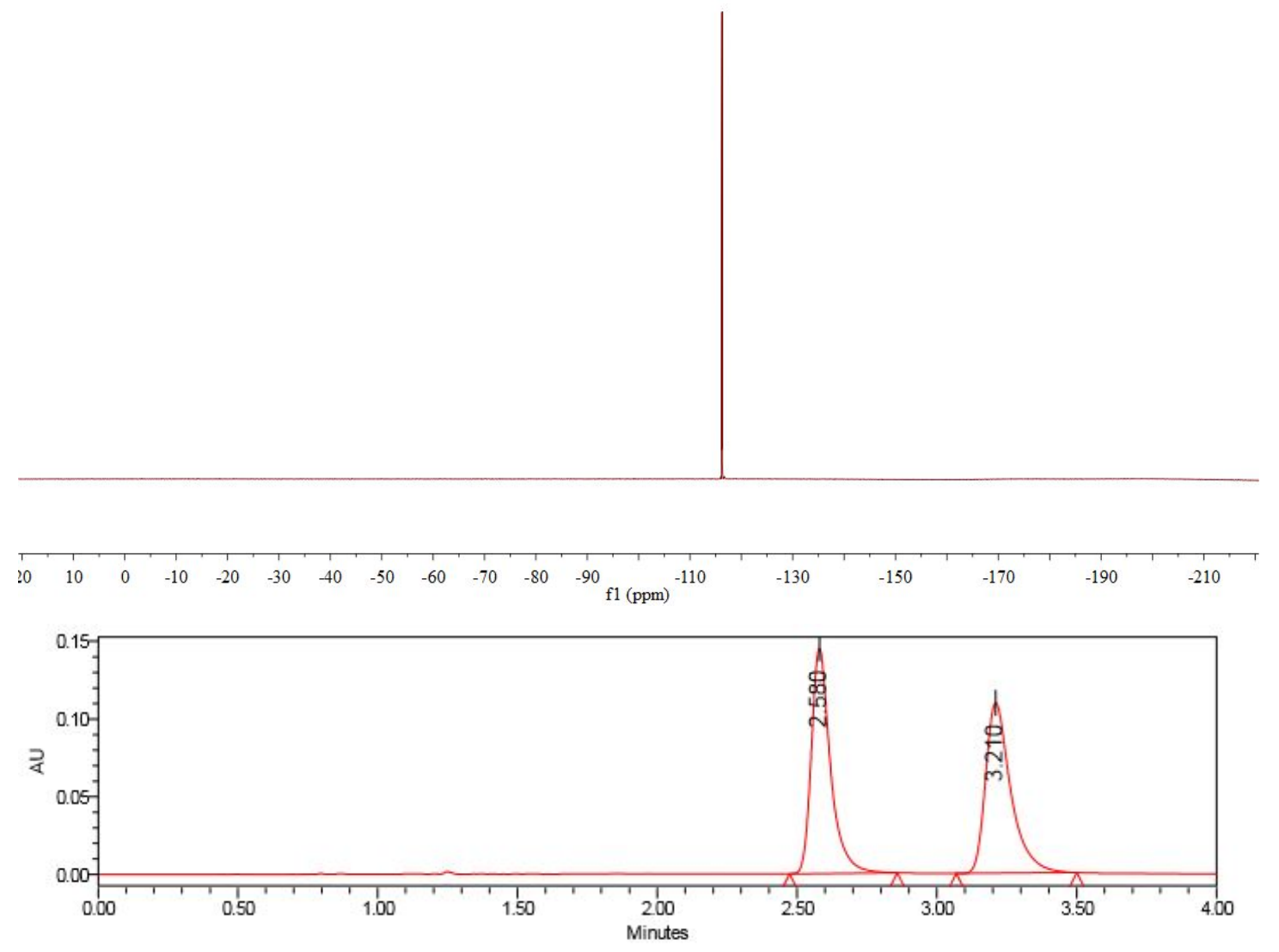

Peak Results
\begin{tabular}{|r|c|c|c|r|}
\hline & RT & Area & Height & $\%$ Area \\
\hline 1 & 2.580 & 690185 & 144824 & 50.03 \\
\hline 2 & 3.210 & 689243 & 109553 & 49.97 \\
\hline
\end{tabular}

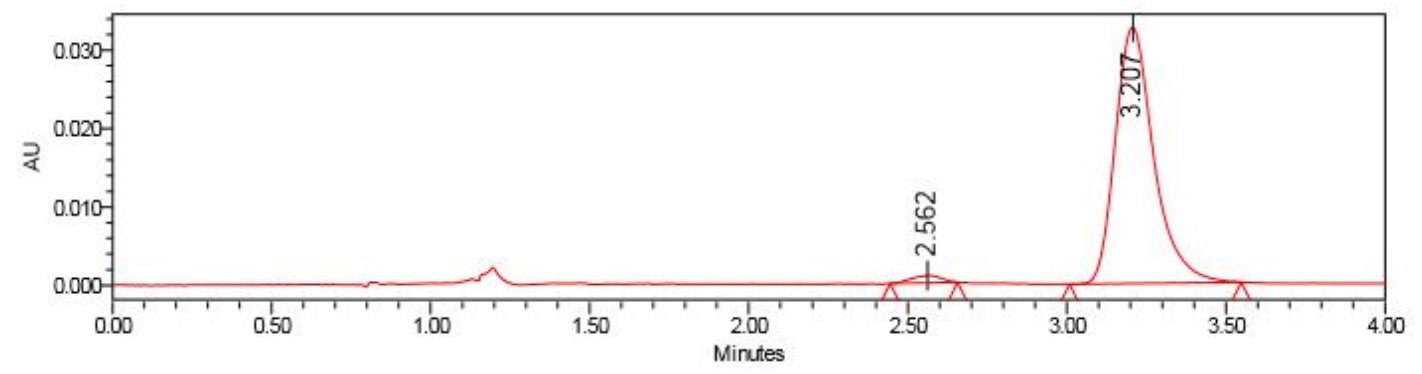

Peak Results

\begin{tabular}{|r|c|r|r|r|}
\hline & RT & Area & Height & $\%$ Area \\
\hline 1 & 2.562 & 6013 & 914 & 214 \\
\hline 2 & 3.207 & 274595 & 32685 & 97.86 \\
\hline
\end{tabular}


(2R,3S,3aR,9bS)-3-(2-chlorophenyl)-9b-methyl-2-(4-nitrophenyl)-2,3,3a,9b-tetrah ydrochromeno[4,3-b]pyrrol-4(1H)-one (3c):

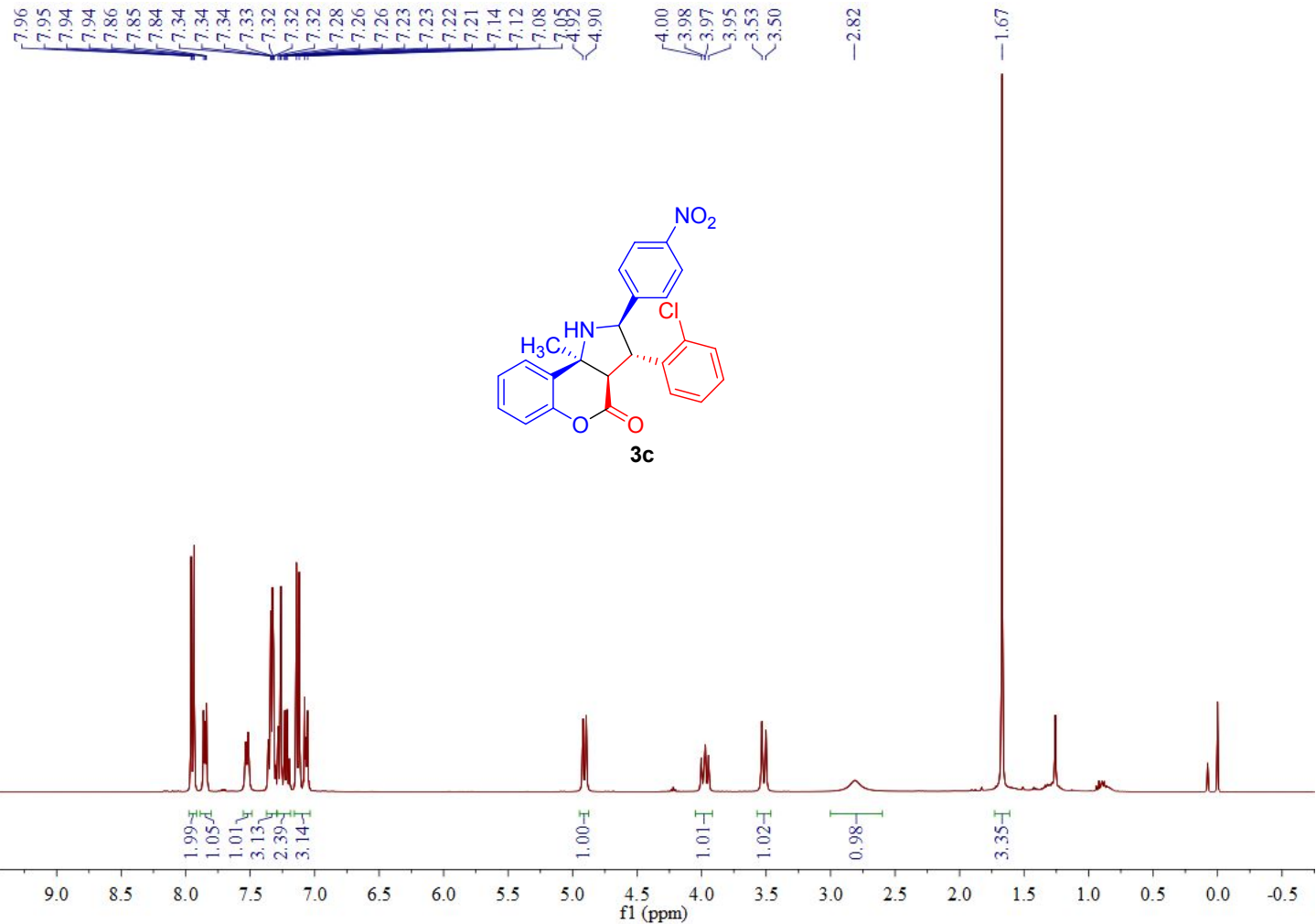

ڤ.

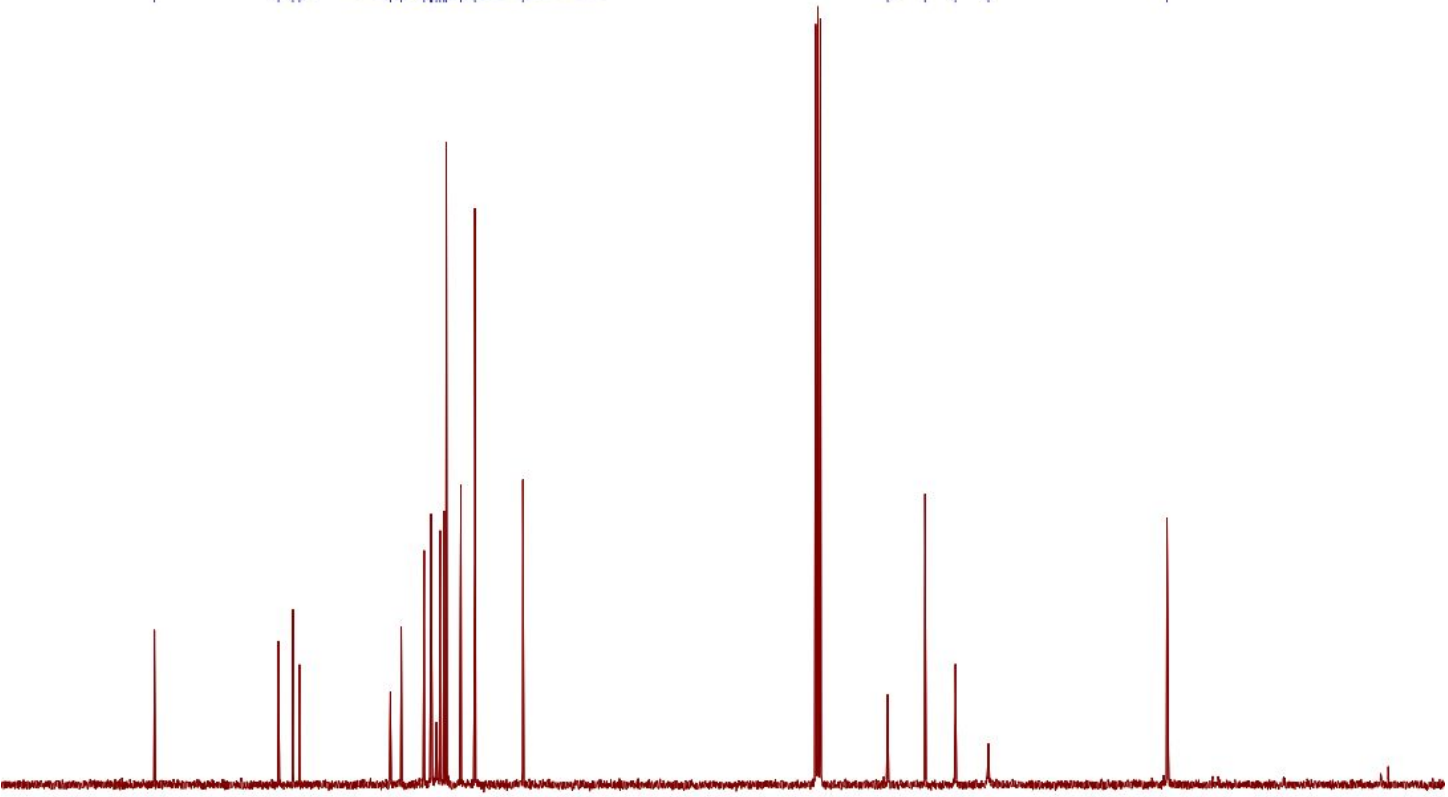

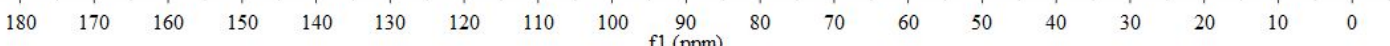




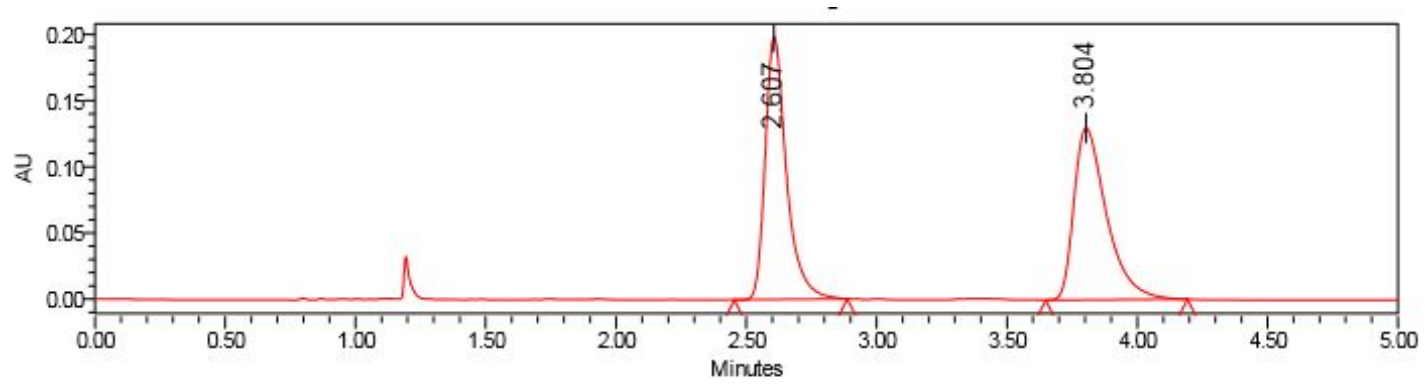

Peak Results

\begin{tabular}{|c|c|c|c|r|}
\hline & RT & Area & Height & $\%$ Area \\
\hline 1 & 2.607 & 1124946 & 198198 & 49.96 \\
\hline 2 & 3.804 & 1126851 & 129639 & 50.04 \\
\hline
\end{tabular}

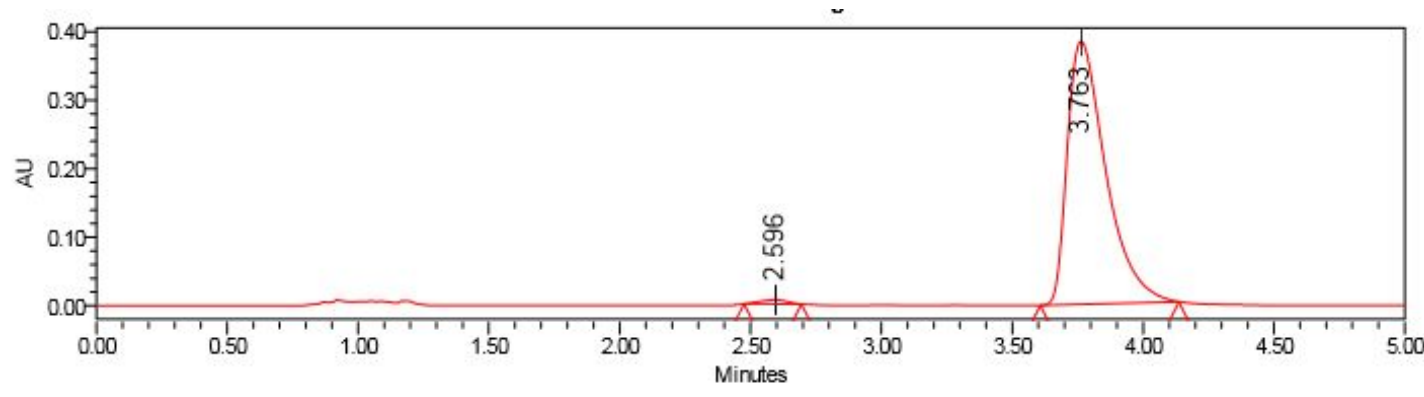

Peak Results

\begin{tabular}{|r|c|r|r|r|}
\hline & RT & Area & Height & $\%$ Area \\
\hline 1 & 2.596 & 43958 & 6058 & 1.11 \\
\hline 2 & 3.763 & 3921478 & 384159 & 98.89 \\
\hline
\end{tabular}


(2R,3S,3aR,9bS)-3-(2-bromophenyl)-9b-methyl-2-(4-nitrophenyl)-2,3,3a,9b-tetra hydrochromeno[4,3-b]pyrrol-4(1H)-one (3d):

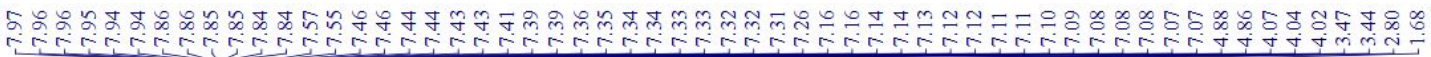
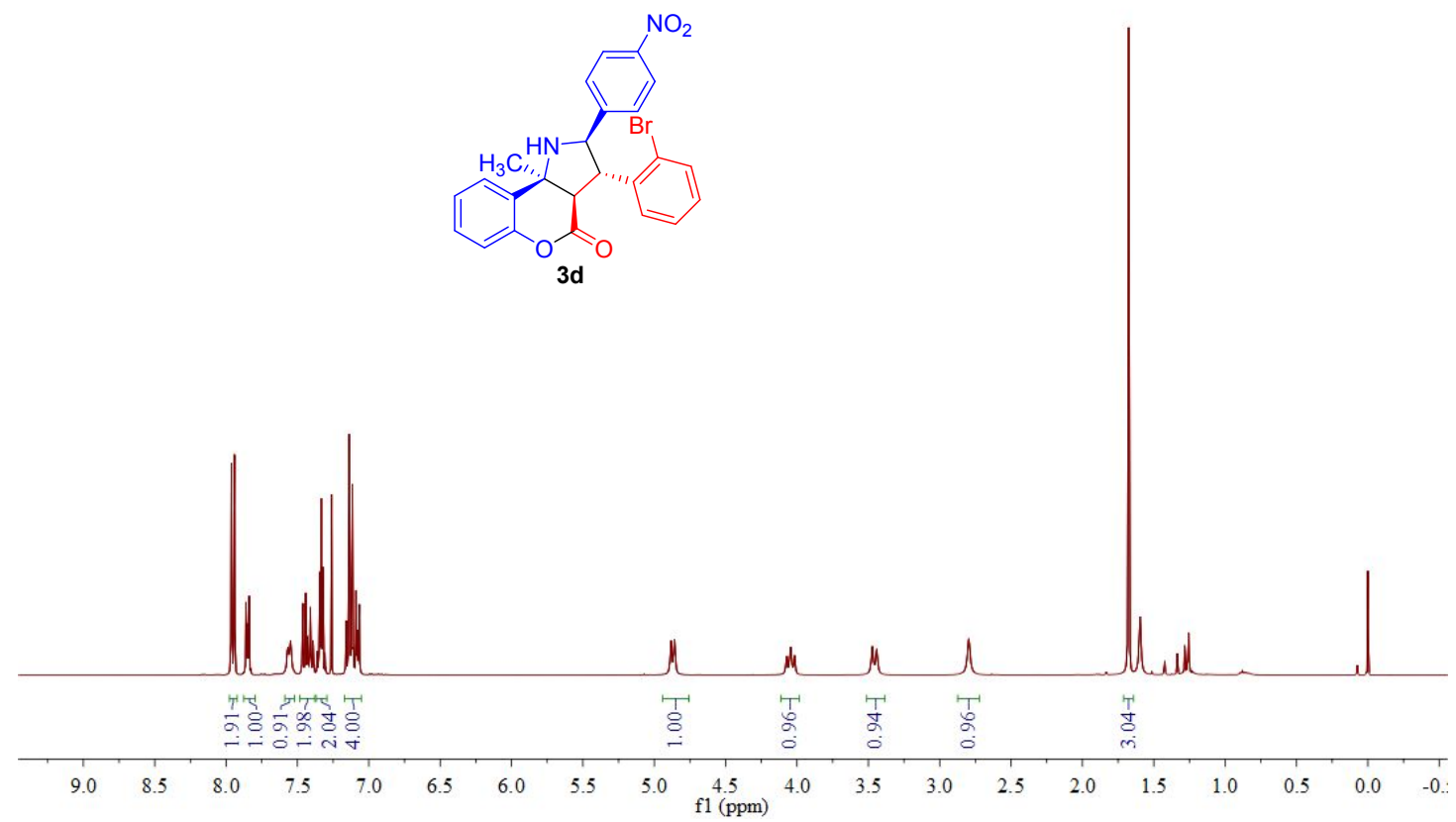

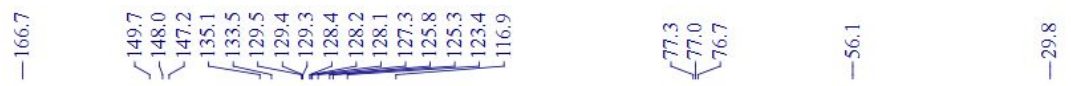

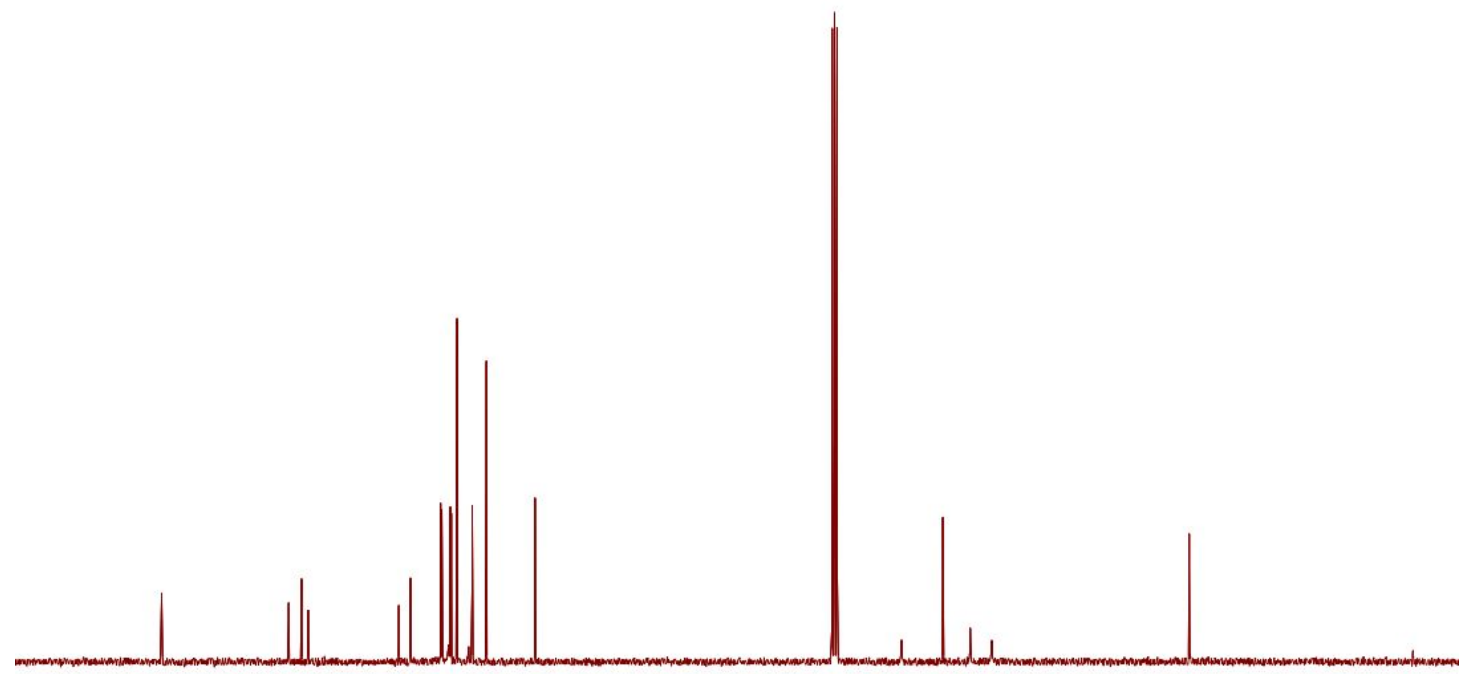

$\begin{array}{lllllllllllllllllll}180 & 170 & 160 & 150 & 140 & 130 & 120 & 110 & 100 & 90 & 80 & 70 & 60 & 50 & 40 & 30 & 20 & 10 & 0\end{array}$ 


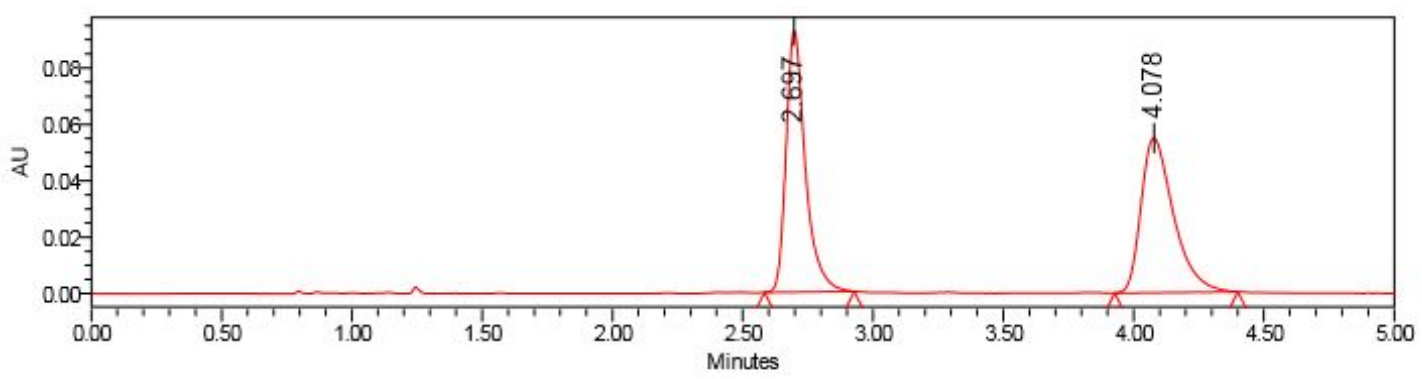

Peak Results

\begin{tabular}{|c|c|c|r|r|}
\hline & RT & Area & Height & $\%$ Area \\
\hline 1 & 2.697 & 476144 & 92760 & 50.06 \\
\hline 2 & 4.078 & 474964 & 54786 & 49.94 \\
\hline
\end{tabular}

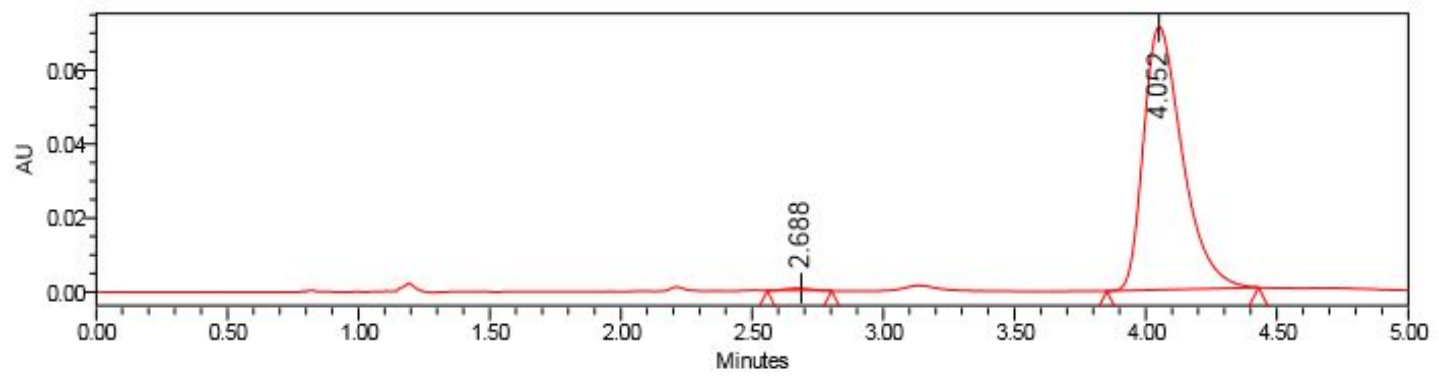

Peak Results

\begin{tabular}{|r|c|r|r|r|}
\hline & RT & Area & Height & $\%$ Area \\
\hline 1 & 2.688 & 4435 & 678 & 0.60 \\
\hline 2 & 4.052 & 733803 & 71171 & 99.40 \\
\hline
\end{tabular}


(2R,3S,3aR,9bS)-3-(2-methoxyphenyl)-9b-methyl-2-(4-nitrophenyl)-2,3,3a,9b-tetr ahydrochromeno $[4,3-b]$ pyrrol-4(1H)-one (3e):

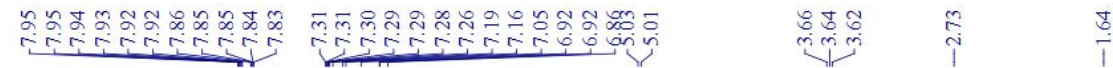
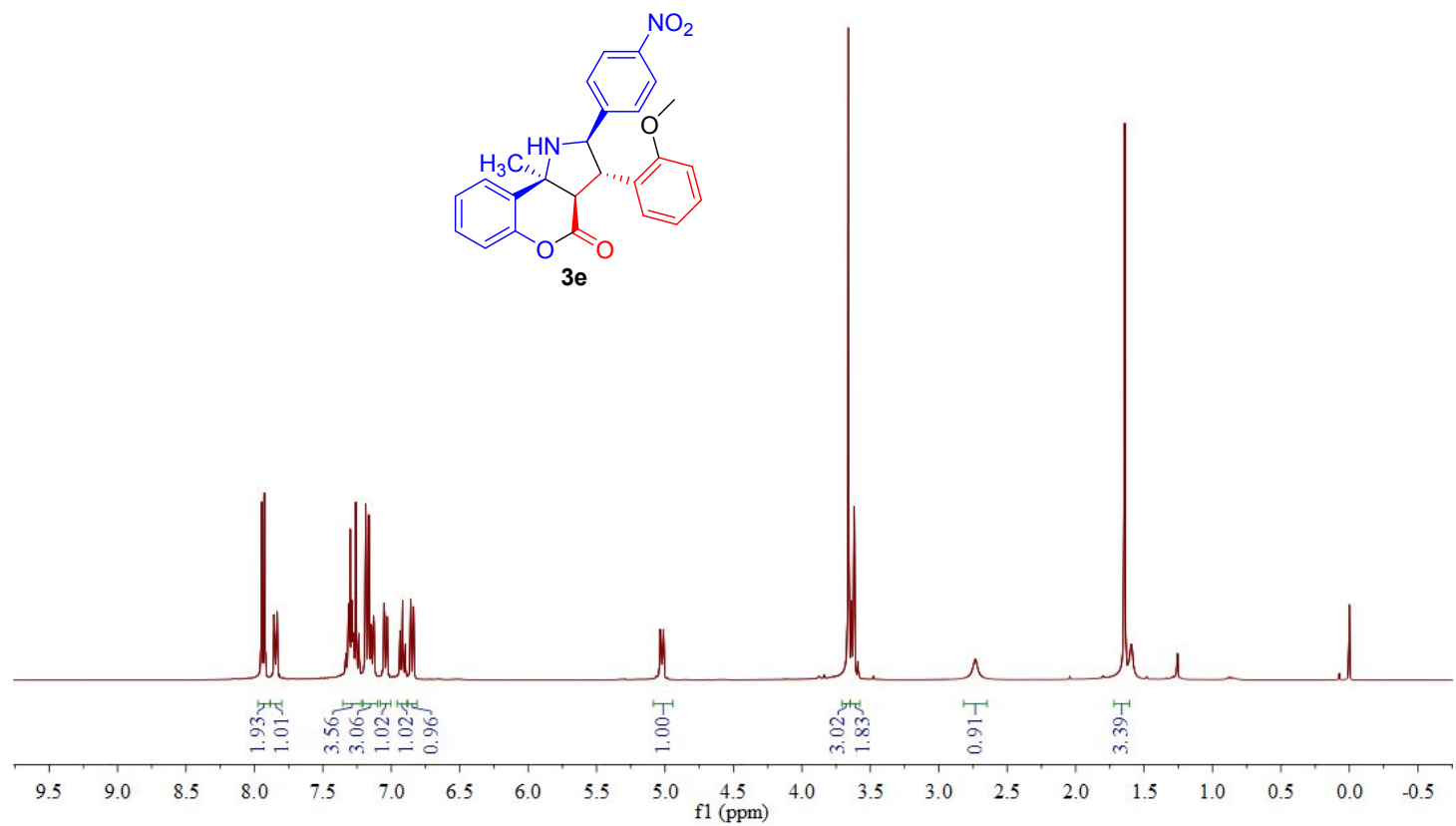

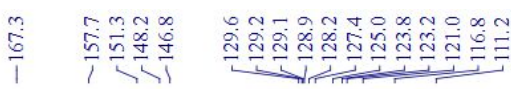

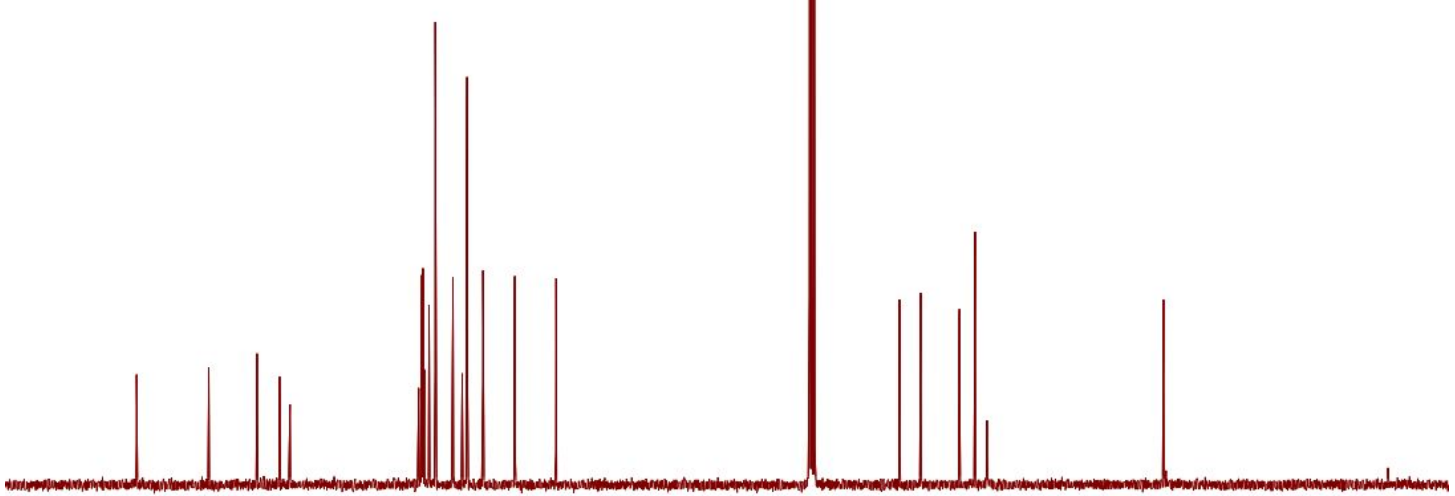

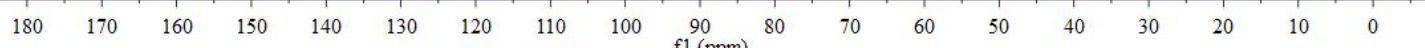




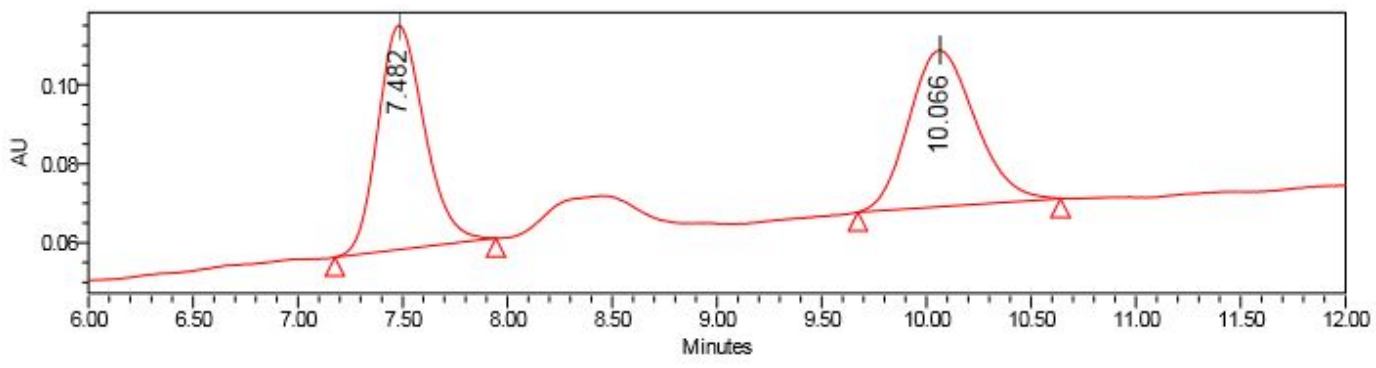

Peak Results

\begin{tabular}{|l|r|r|r|r|}
\hline & \multicolumn{1}{|c|}{ RT } & Area & Height & \% Area \\
\hline 1 & 7.482 & 872554 & 56768 & 50.04 \\
\hline 2 & 10.066 & 871040 & 39629 & 49.96 \\
\hline
\end{tabular}

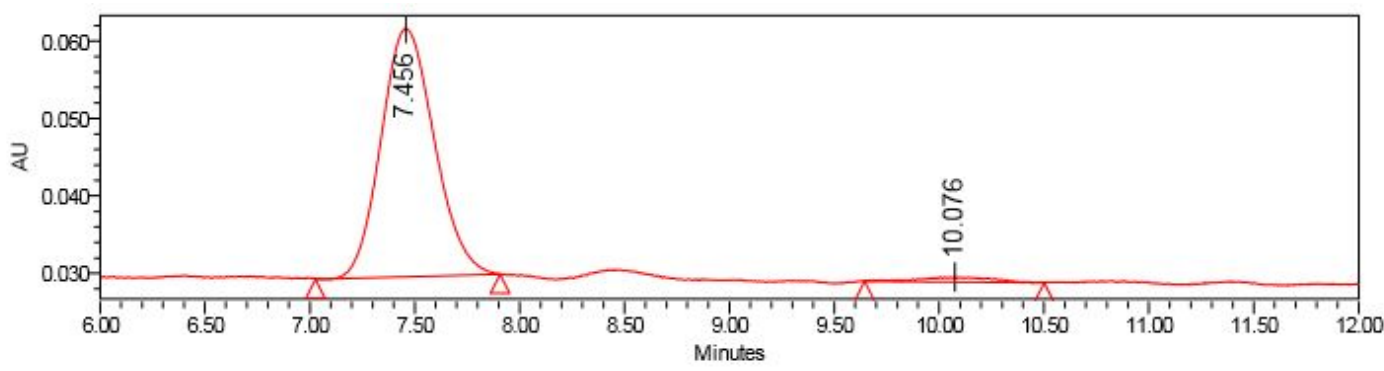

Peak Results

\begin{tabular}{|l|c|r|r|r|}
\hline & RT & Area & Height & $\%$ Area \\
\hline 1 & 7.456 & 566369 & 32074 & 97.43 \\
\hline 2 & 10.076 & 14927 & 644 & 2.57 \\
\hline
\end{tabular}


(2R,3S,3aR,9bS)-3-(3-chlorophenyl)-9b-methyl-2-(4-nitrophenyl)-2,3,3a,9b-tetrah ydrochromeno[4,3-b]pyrrol-4(1H)-one (3f):

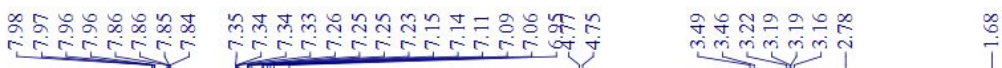
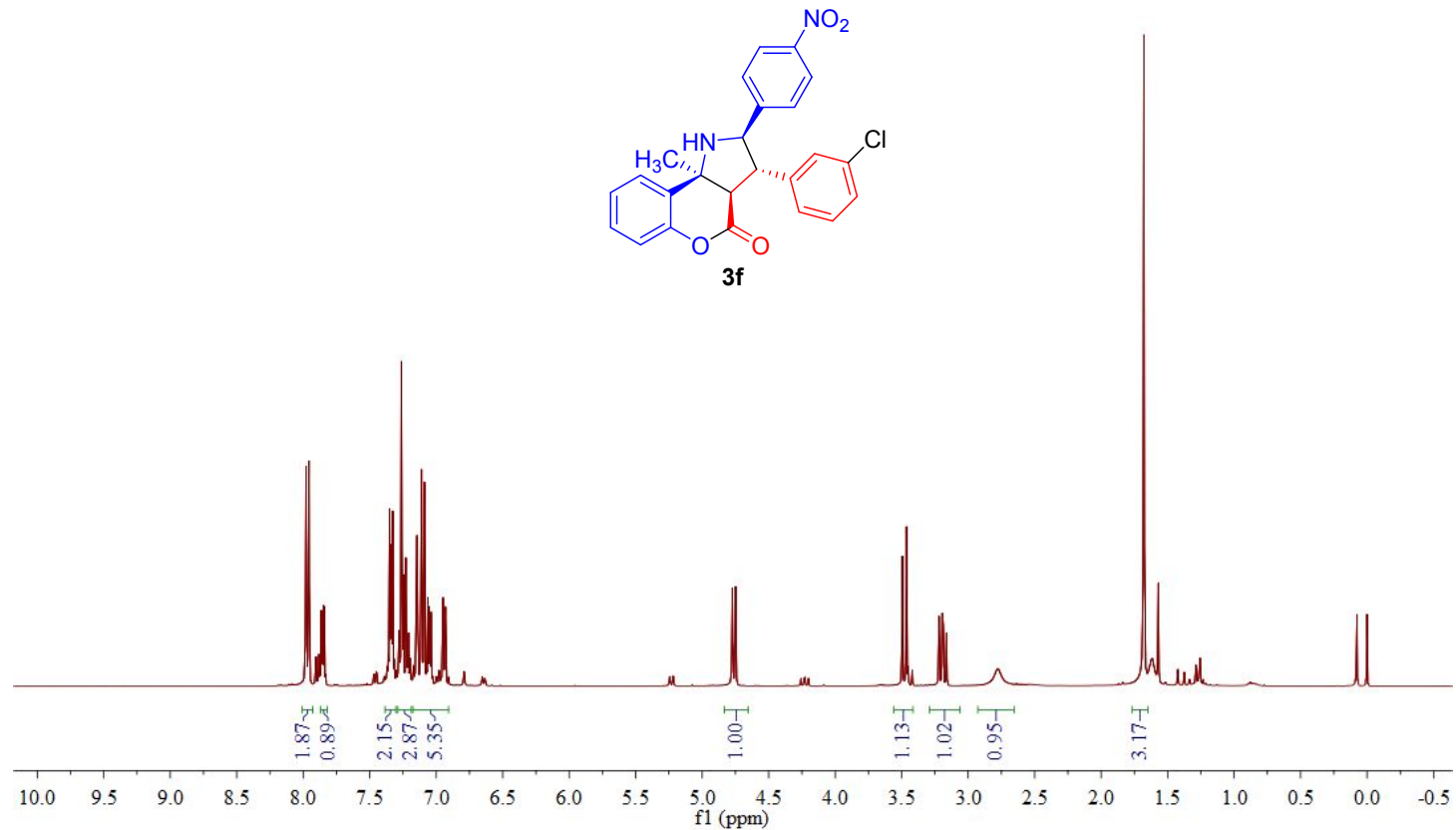

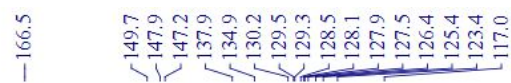

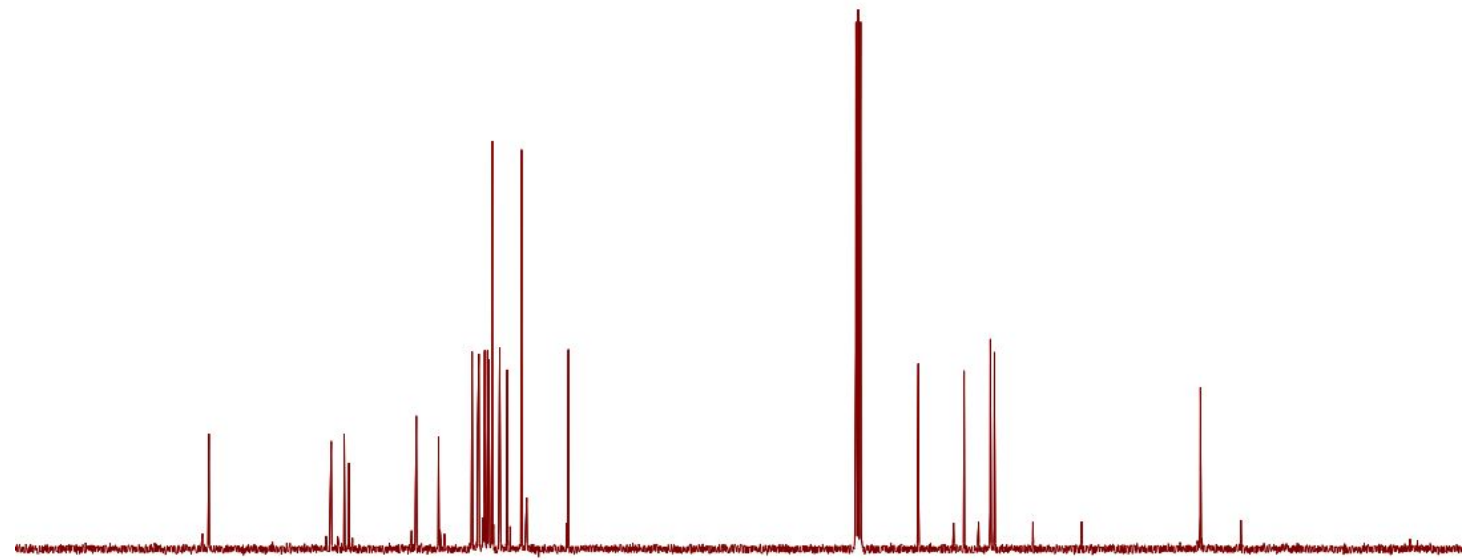

$\begin{array}{llllllllll}190 & 180 & 170 & 160 & 150 & 140 & 130 & 120 & 110 & 100 \\ \mathrm{f} 1(\mathrm{ppm})\end{array}$

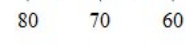

50

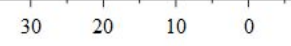




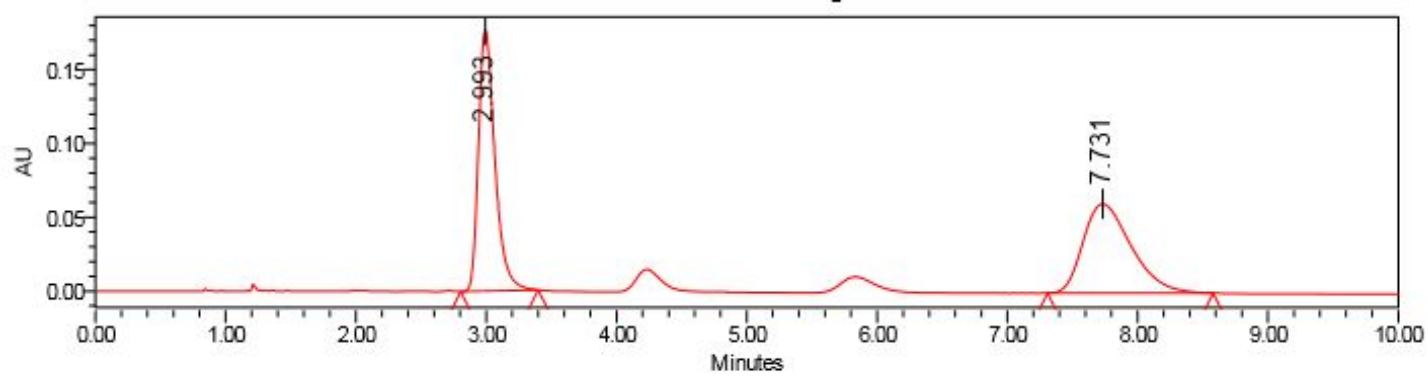

Peak Results

\begin{tabular}{|c|c|c|r|r|}
\hline & RT & Area & Height & \% Area \\
\hline 1 & 2.993 & 1556497 & 176841 & 50.07 \\
\hline 2 & 7.731 & 1552148 & 60484 & 49.93 \\
\hline
\end{tabular}

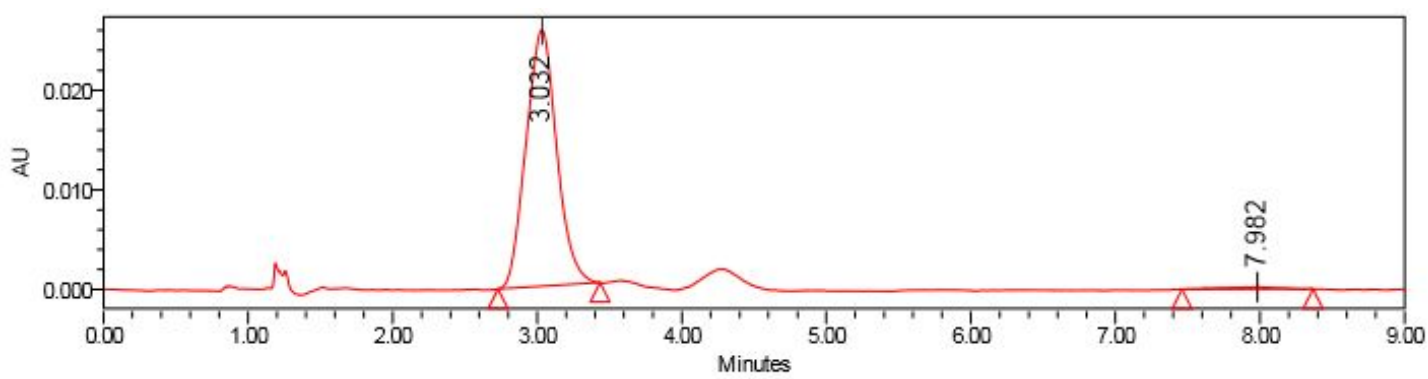

Peak Results

\begin{tabular}{|r|c|r|r|r|}
\hline & RT & Area & Height & \% Area \\
\hline 1 & 3.032 & 380074 & 25721 & 96.48 \\
\hline 2 & 7.982 & 5877 & 231 & 1.52 \\
\hline
\end{tabular}


(2R,3S,3aR,9bS)-9b-methyl-2-(4-nitrophenyl)-3-(3-(trifluoromethyl)phenyl)-2,3,3 a,9b-tetrahydrochromeno[4,3-b]pyrrol-4(1H)-one (3g):

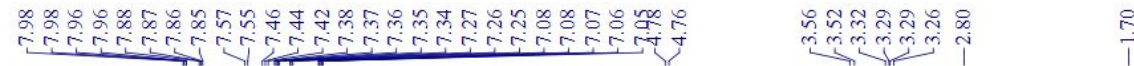
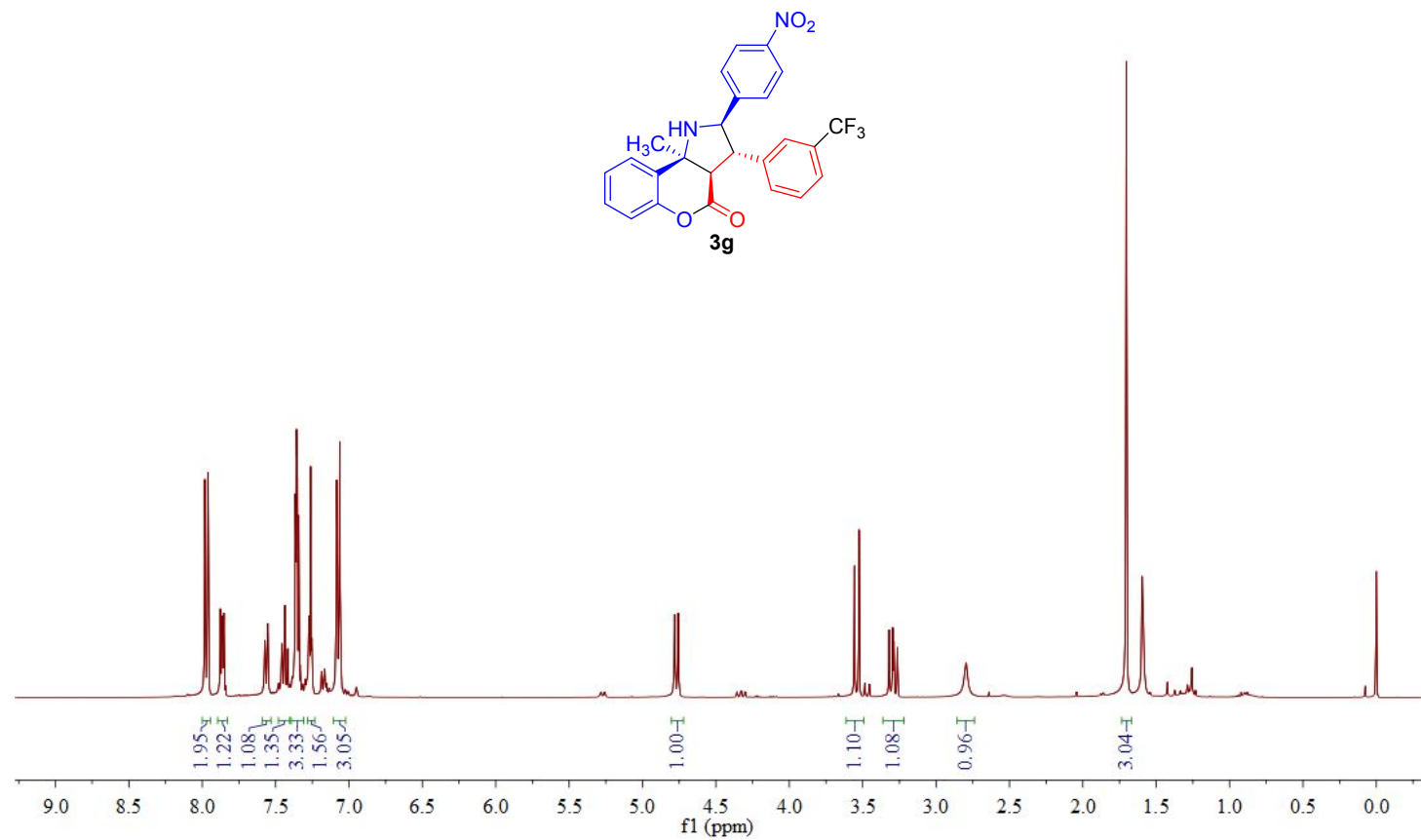

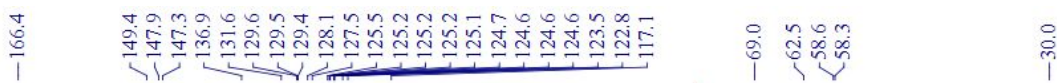

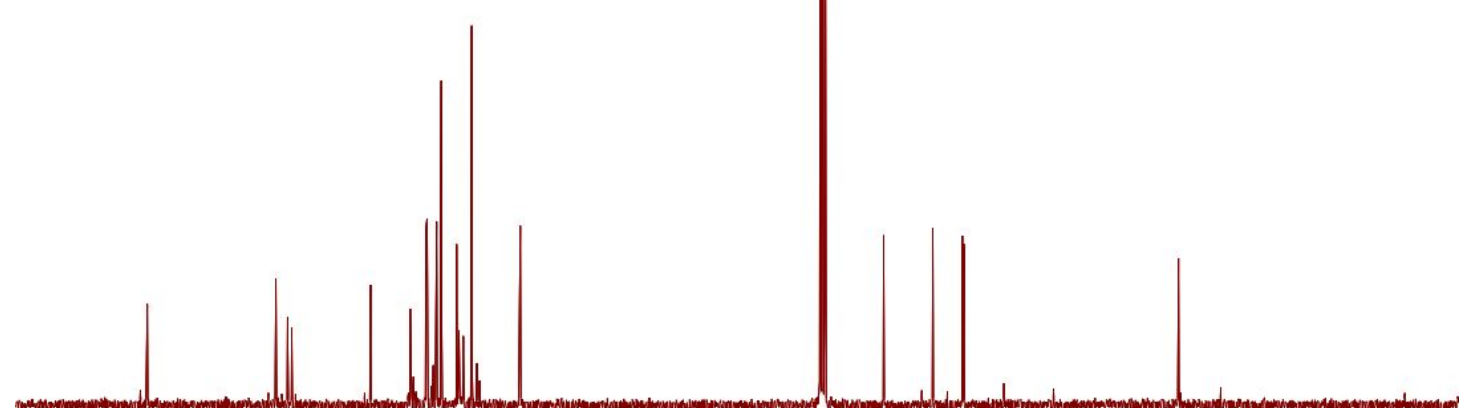

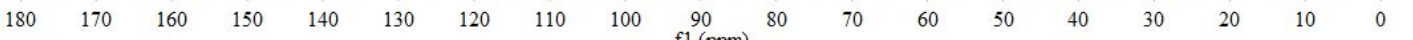



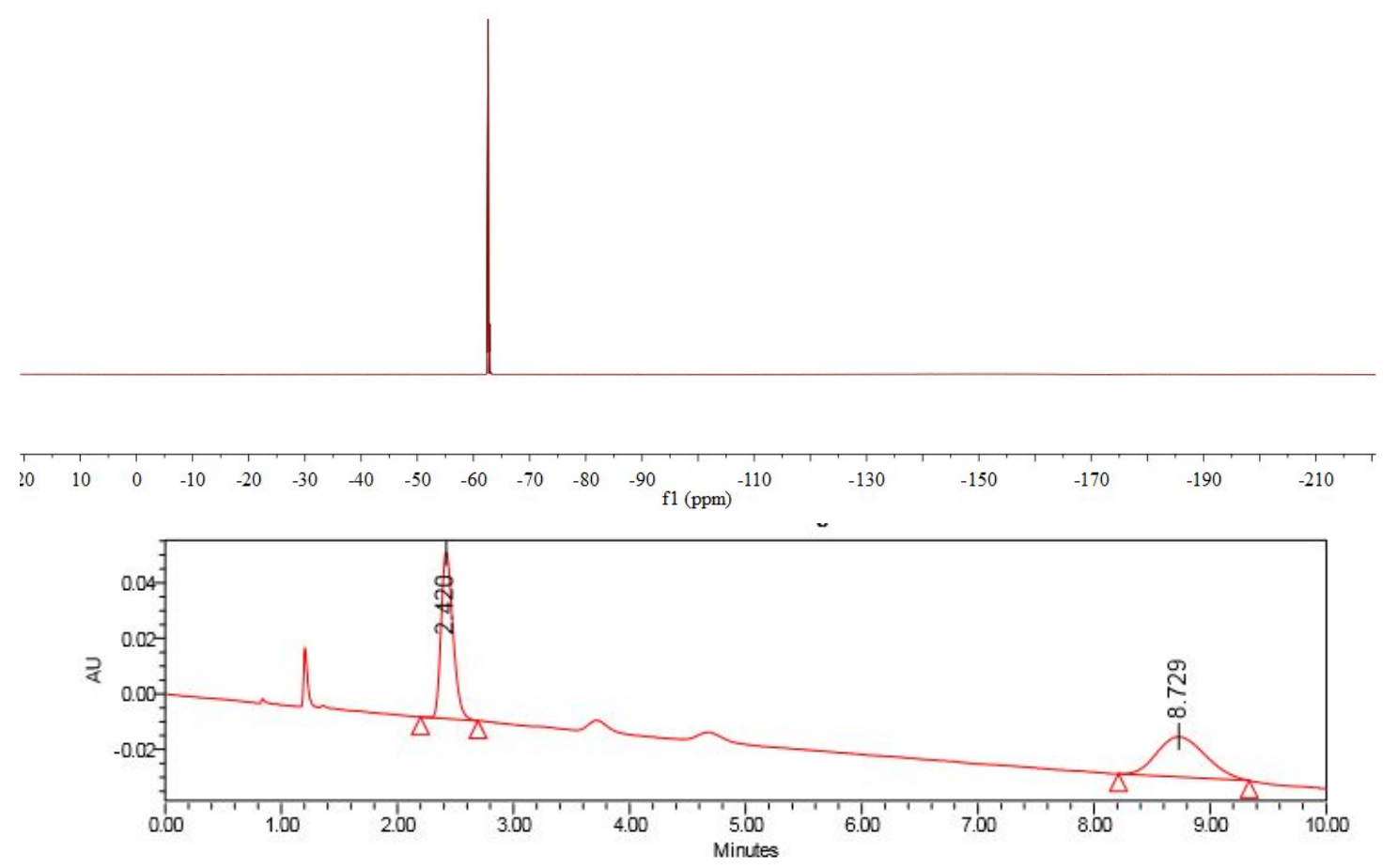

Peak Results

\begin{tabular}{|r|c|c|r|r|}
\hline & RT & Area & Height & \% Area \\
\hline 1 & 2.420 & 424386 & 59914 & 50.15 \\
\hline 2 & 8.729 & 421835 & 14544 & 49.85 \\
\hline
\end{tabular}

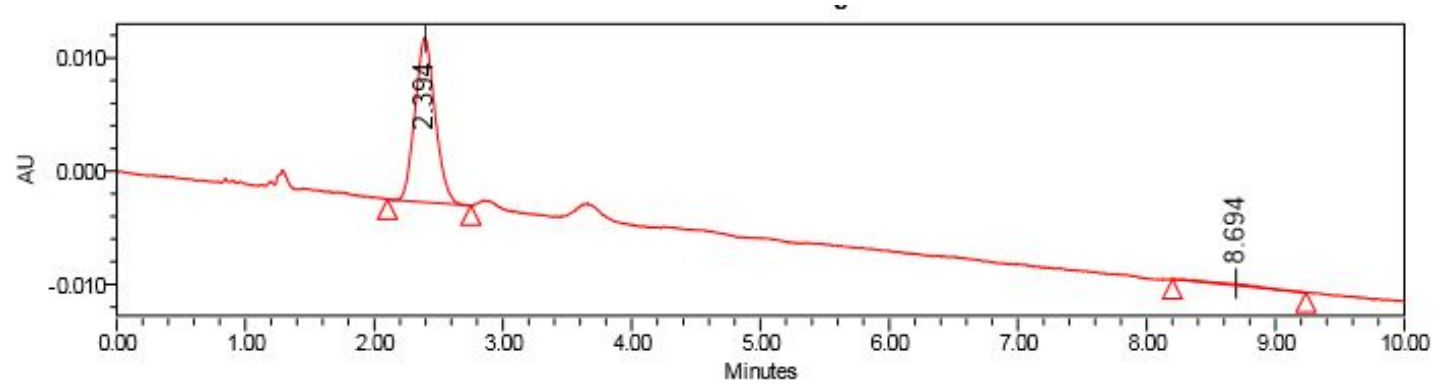

Peak Results

\begin{tabular}{|r|c|c|r|r|}
\hline & RT & Area & Height & $\%$ Area \\
\hline 1 & 2.394 & 157934 & 14560 & 97.11 \\
\hline 2 & 8.694 & 4707 & 212 & 2.89 \\
\hline
\end{tabular}


(2R,3S,3aR,9bS)-3-(4-fluorophenyl)-9b-methyl-2-(4-nitrophenyl)-2,3,3a,9b-tetrah ydrochromeno[4,3-b]pyrrol-4(1H)-one $(3 \mathrm{~h})$ :

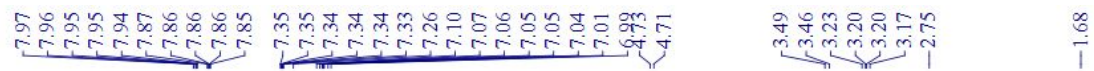
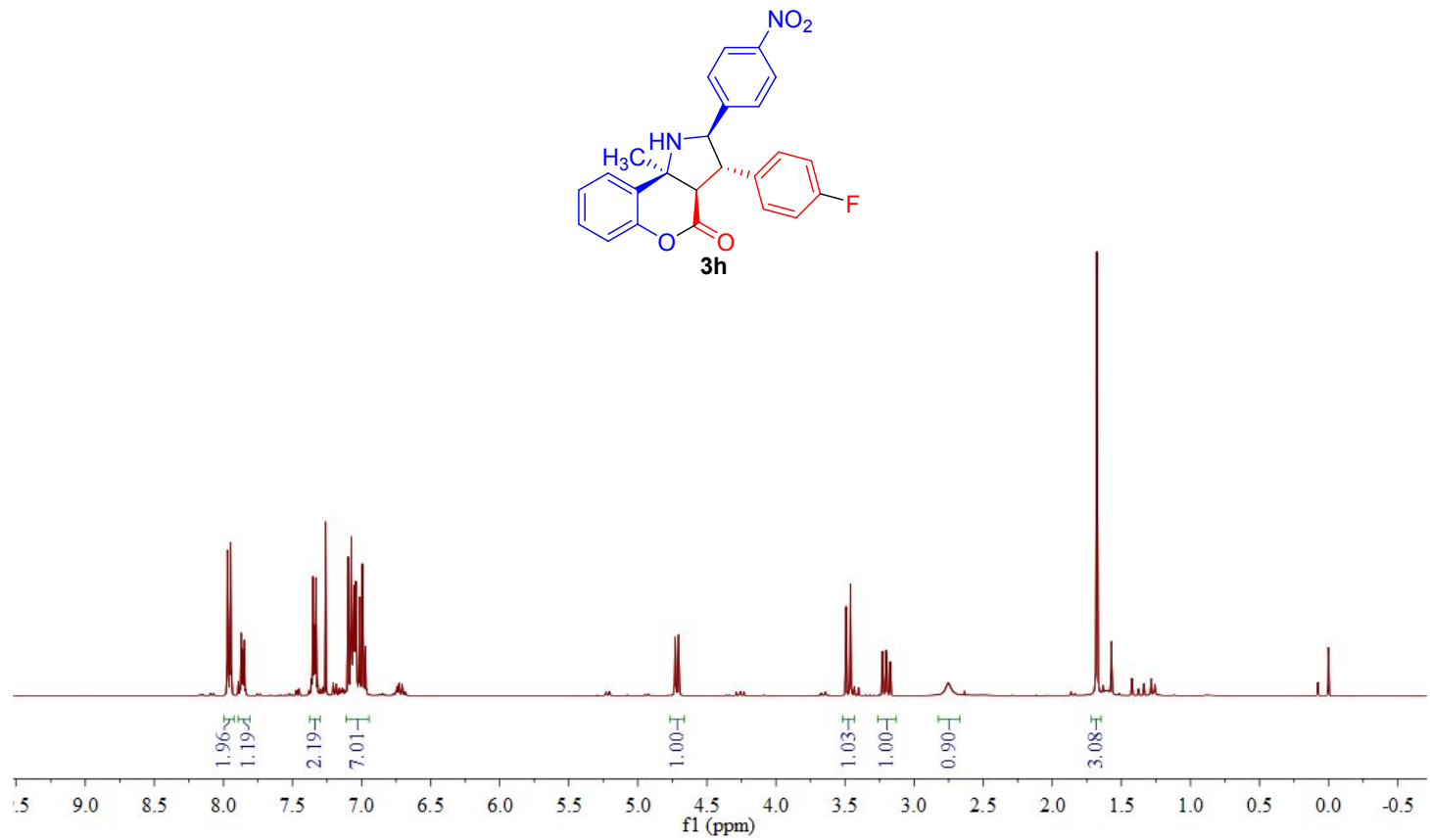

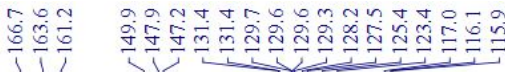

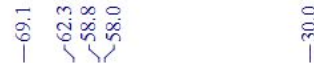

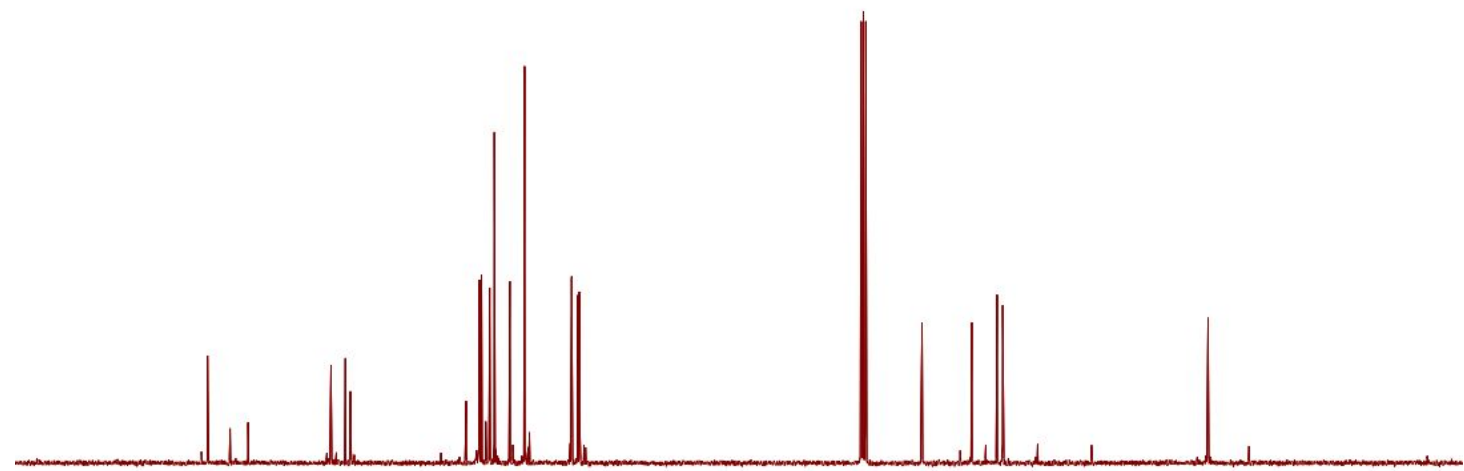

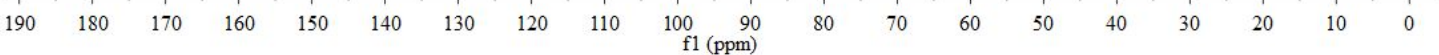




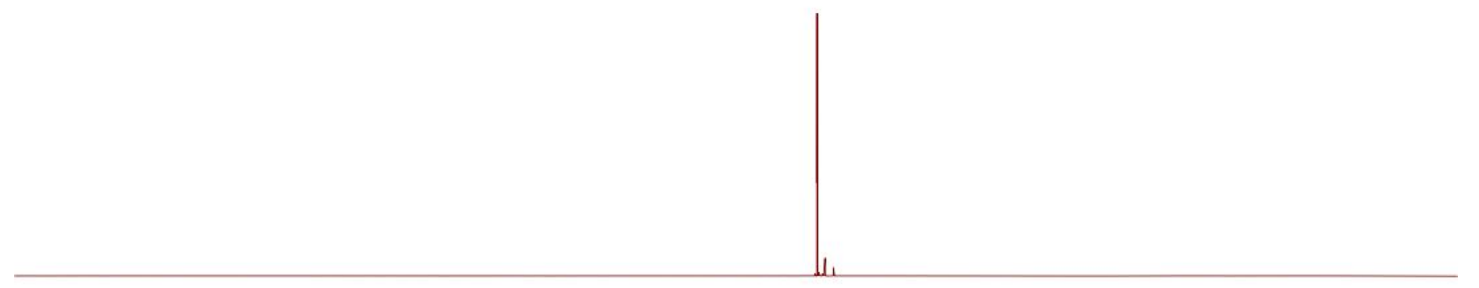

\begin{tabular}{lllllllllllllllllllll}
\hline 0 & 10 & 0 & -10 & -20 & -30 & -40 & -50 & -60 & -70 & -80 & -90 & -110 & -130 & -150 & -170 & -190 & -210 \\
\hline
\end{tabular}

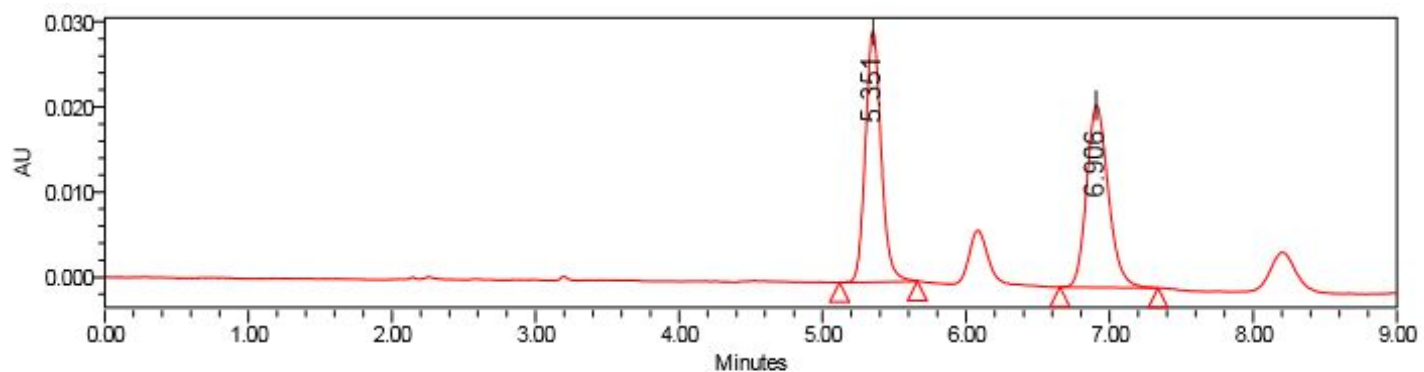

Peak Results

\begin{tabular}{|c|c|c|r|r|}
\hline & RT & Area & Height & $\%$ Area \\
\hline 1 & 5.351 & 225355 & 29466 & 50.06 \\
\hline 2 & 6.906 & 224783 & 21375 & 49.94 \\
\hline
\end{tabular}

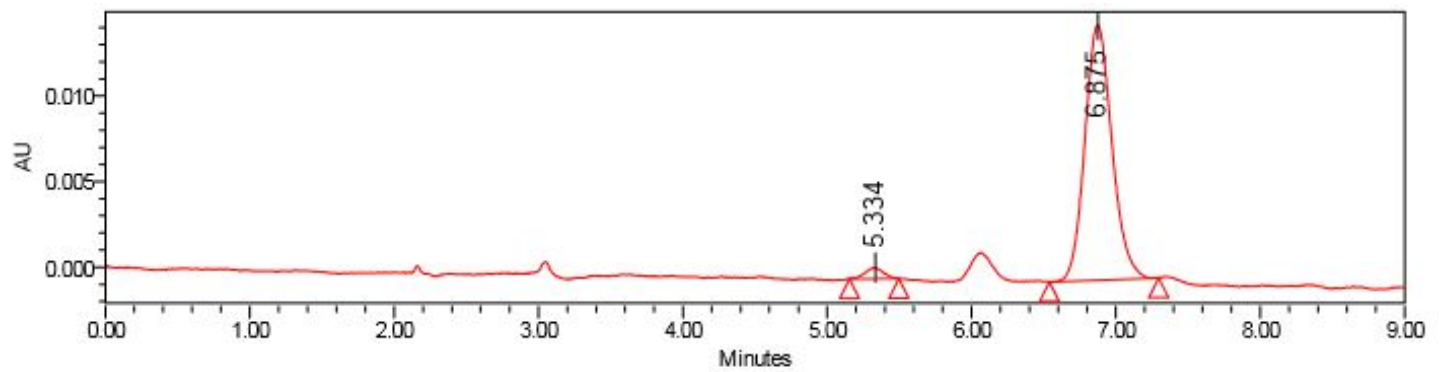

Peak Results

\begin{tabular}{|r|c|r|r|r|}
\hline & RT & Area & Height & \% Area \\
\hline 1 & 5.334 & 5765 & 656 & 2.88 \\
\hline 2 & 6.875 & 194283 & 14907 & 97.12 \\
\hline
\end{tabular}


(2R,3S,3aR,9bS)-3-(4-chlorophenyl)-9b-methyl-2-(4-nitrophenyl)-2,3,3a,9b-tetrah ydrochromeno[4,3-b]pyrrol-4(1H)-one (3i):

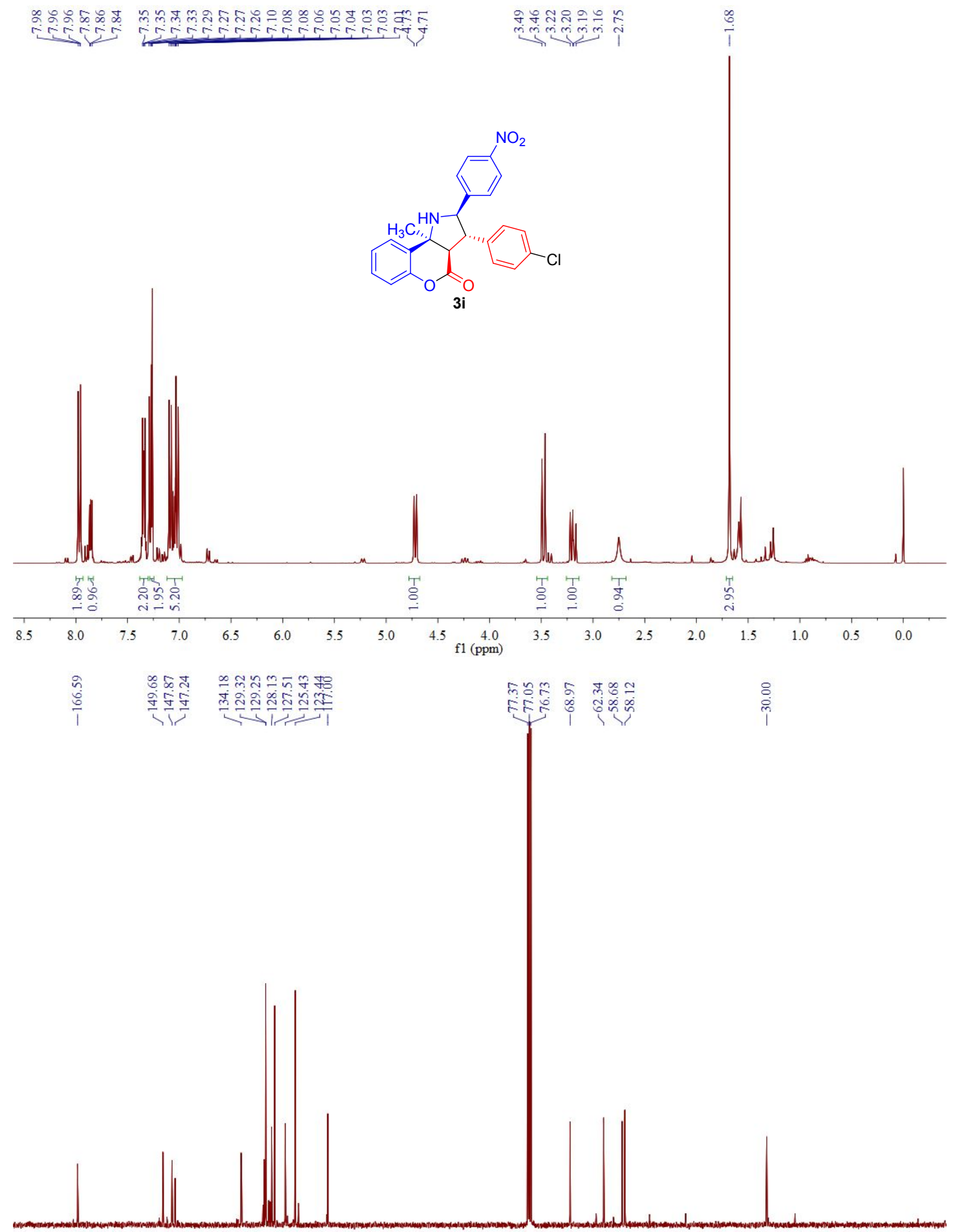

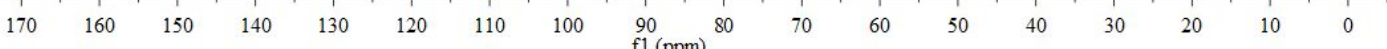




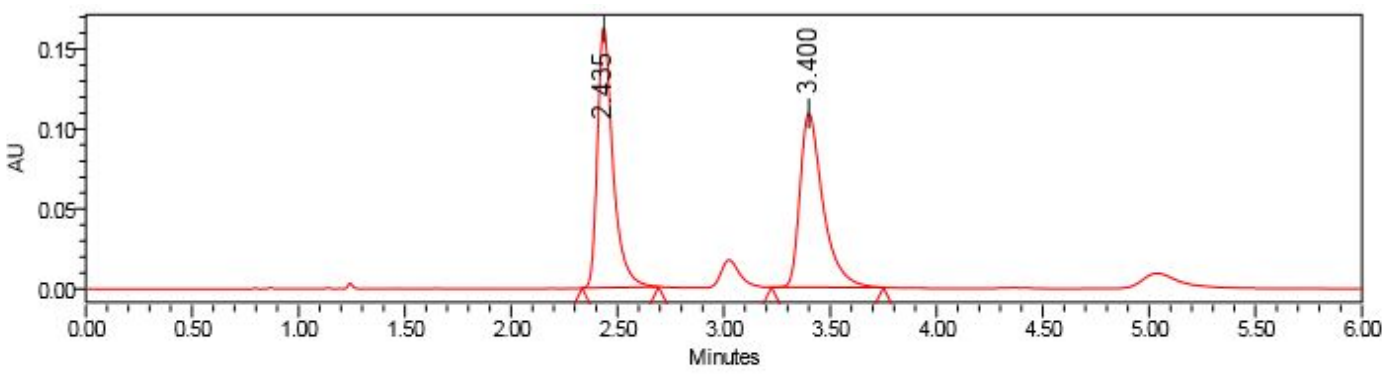

Peak Results

\begin{tabular}{|c|c|c|c|r|}
\hline & RT & Area & Height & \% Area \\
\hline 1 & 2.435 & 822341 & 162531 & 50.11 \\
\hline 2 & 3.400 & 818716 & 108703 & 49.89 \\
\hline
\end{tabular}

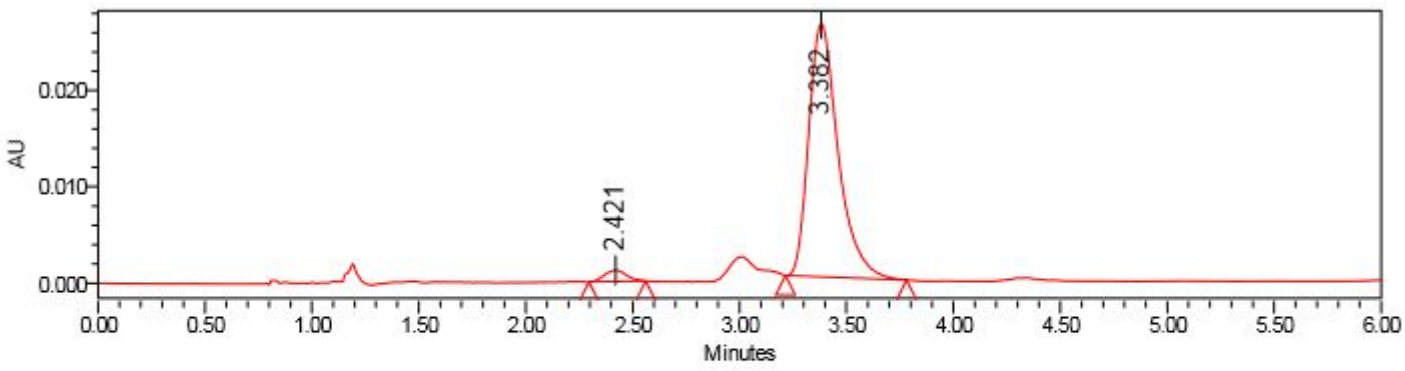

Peak Results

\begin{tabular}{|r|c|r|r|r|}
\hline & RT & Area & Height & \% Area \\
\hline 1 & 2.421 & 8230 & 1162 & 3.17 \\
\hline 2 & 3.382 & 251387 & 26274 & 96.83 \\
\hline
\end{tabular}


(2R,3S,3aR,9bS)-9b-methyl-2,3-bis(4-nitrophenyl)-2,3,3a,9b-tetrahydrochromeno [4,3-b]pyrrol-4(1H)-one (3j):

$\frac{a}{\infty} \frac{\infty}{\infty}=8 g \infty \infty \infty \infty$

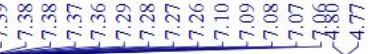

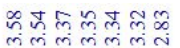

$\stackrel{+}{\stackrel{1}{1}}$

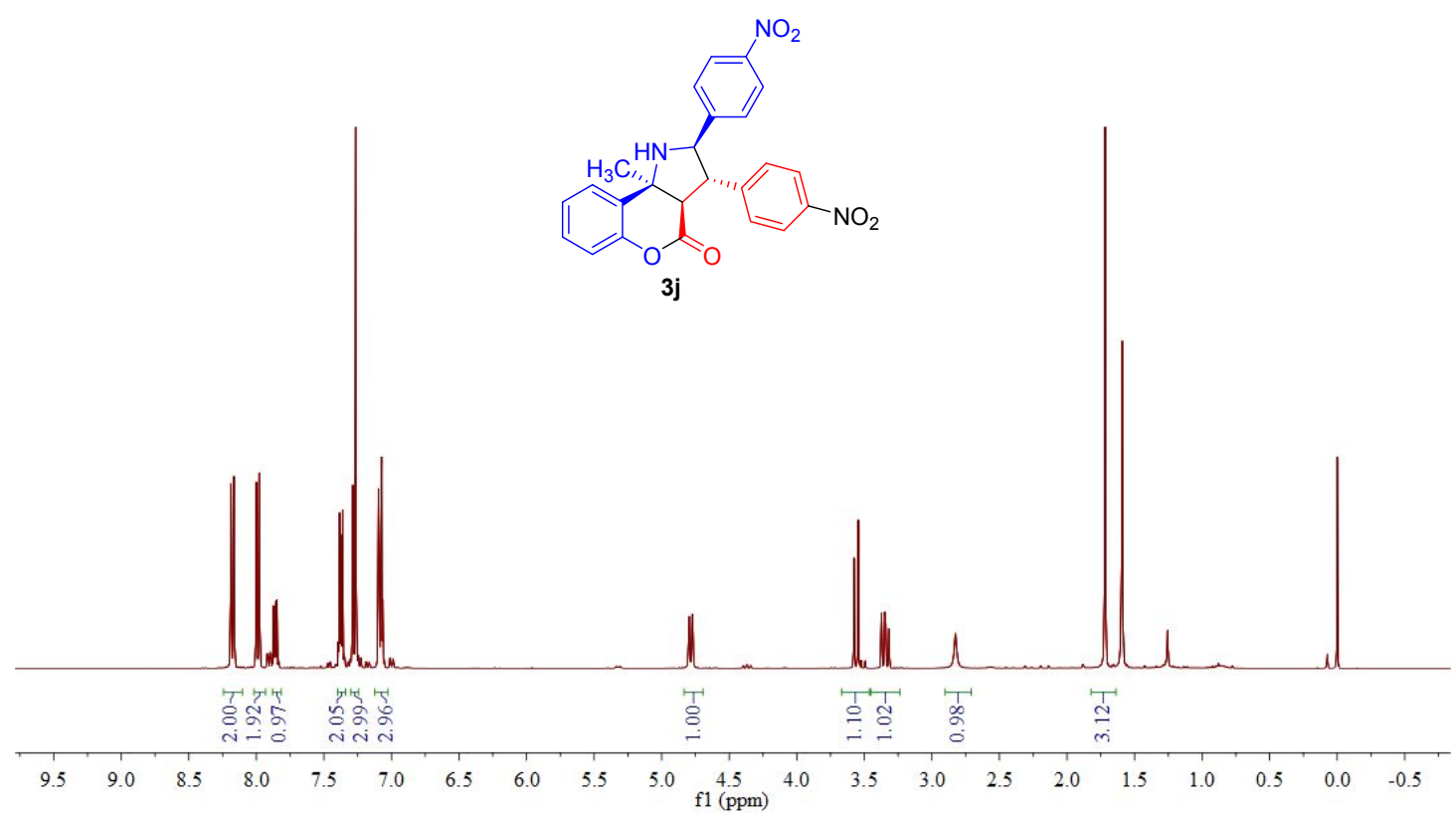

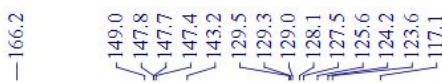

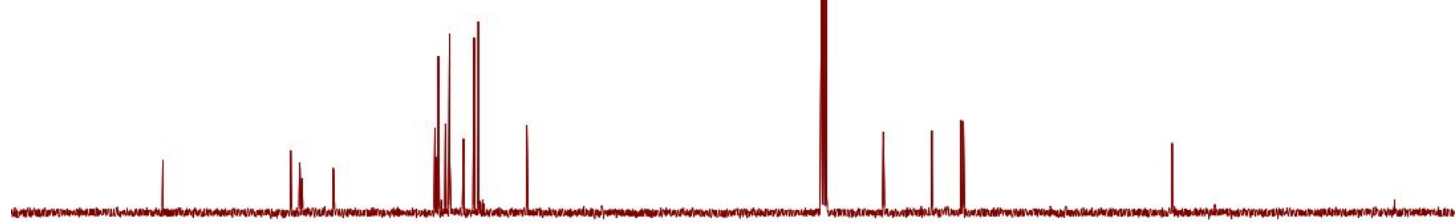

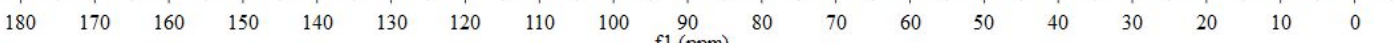




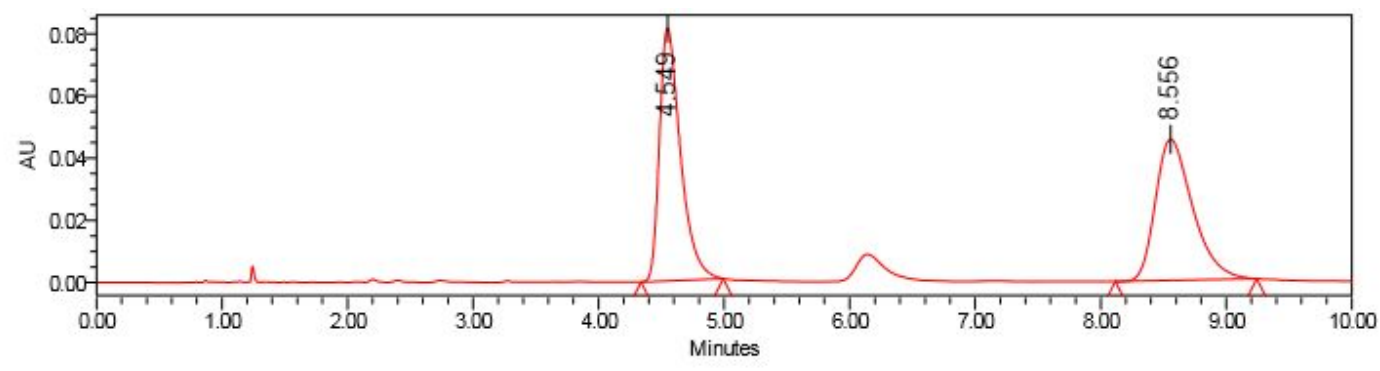

Peak Results

\begin{tabular}{|c|c|c|r|r|}
\hline & RT & Area & Height & \% Area \\
\hline 1 & 4.549 & 947174 & 81418 & 50.21 \\
\hline 2 & 8.556 & 939254 & 45476 & 49.79 \\
\hline
\end{tabular}

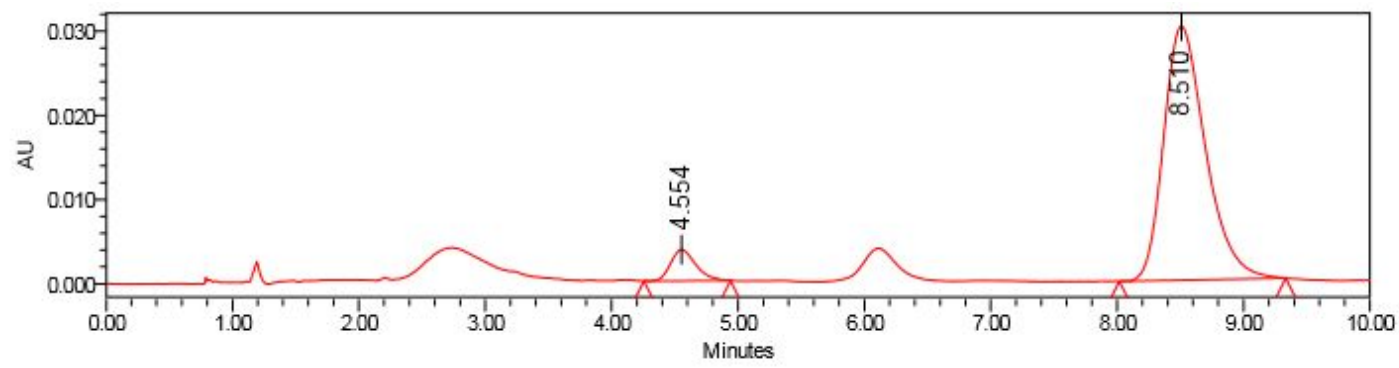

Peak Results

\begin{tabular}{|r|c|c|r|r|}
\hline & RT & Area & Height & $\%$ Area \\
\hline 1 & 4.554 & 50864 & 3703 & 6.82 \\
\hline 2 & 8.510 & 695450 & 30190 & 93.18 \\
\hline
\end{tabular}


(2R,3S,3aR,9bS)-9b-methyl-2-(4-nitrophenyl)-3-(p-tolyl)-2,3,3a,9b-tetrahydrochr omeno[4,3-b]pyrrol-4(1H)-one (3k):

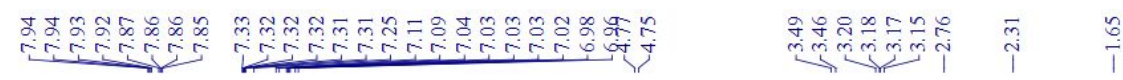
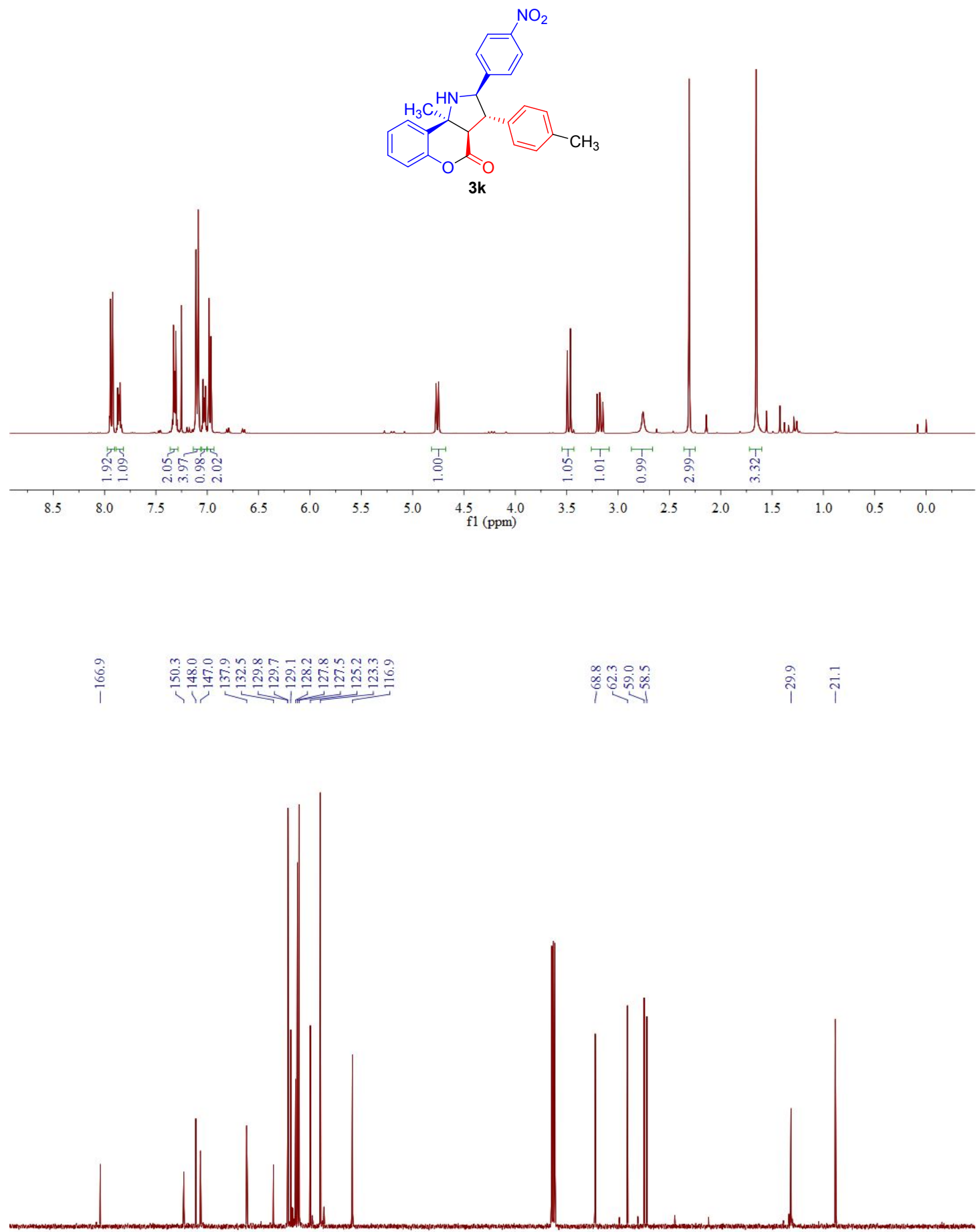


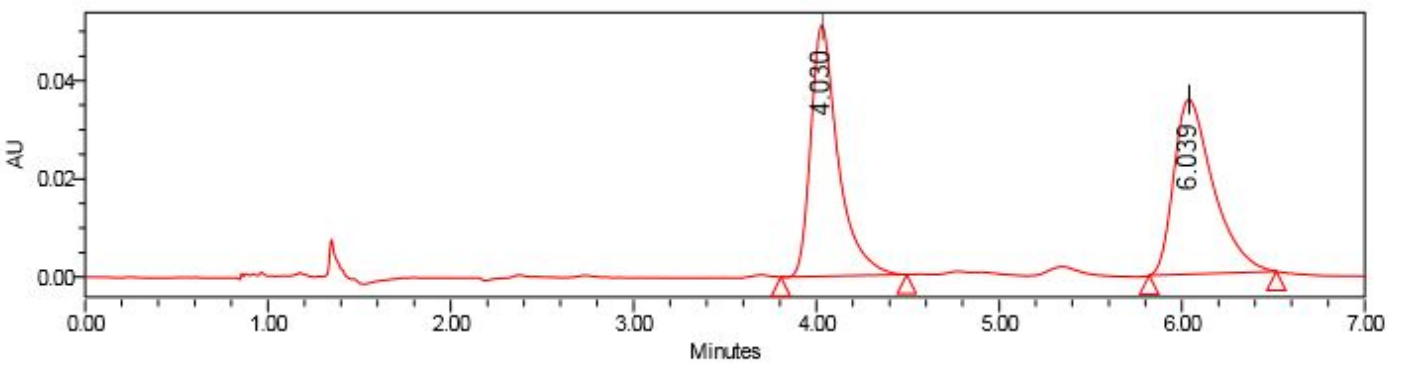

Peak Results

\begin{tabular}{|l|c|c|r|r|}
\hline & RT & Area & Height & \% Area \\
\hline 1 & 4.030 & 531553 & 51095 & 49.90 \\
\hline 2 & 6.039 & 533640 & 35578 & 50.10 \\
\hline
\end{tabular}

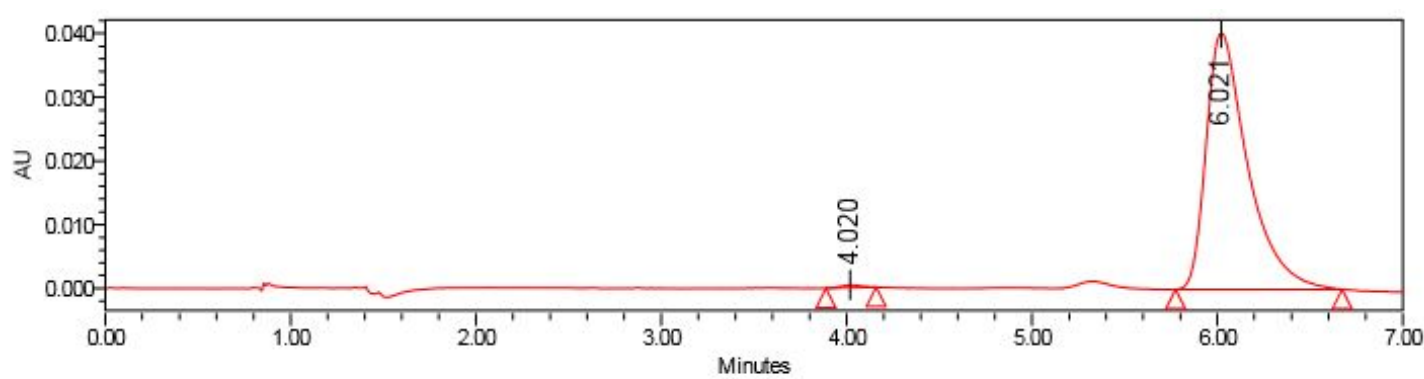

Peak Results

\begin{tabular}{|r|c|r|r|r|}
\hline & RT & Area & Height & $\%$ Area \\
\hline 1 & 4.020 & 3214 & 445 & 0.51 \\
\hline 2 & 6.021 & 626747 & 40262 & 99.49 \\
\hline
\end{tabular}


(2R,3S,3aR,9bS)-3-(4-methoxyphenyl)-9b-methyl-2-(4-nitrophenyl)-2,3,3a,9b-tetr ahydrochromeno[4,3-b]pyrrol-4(1H)-one (3l):
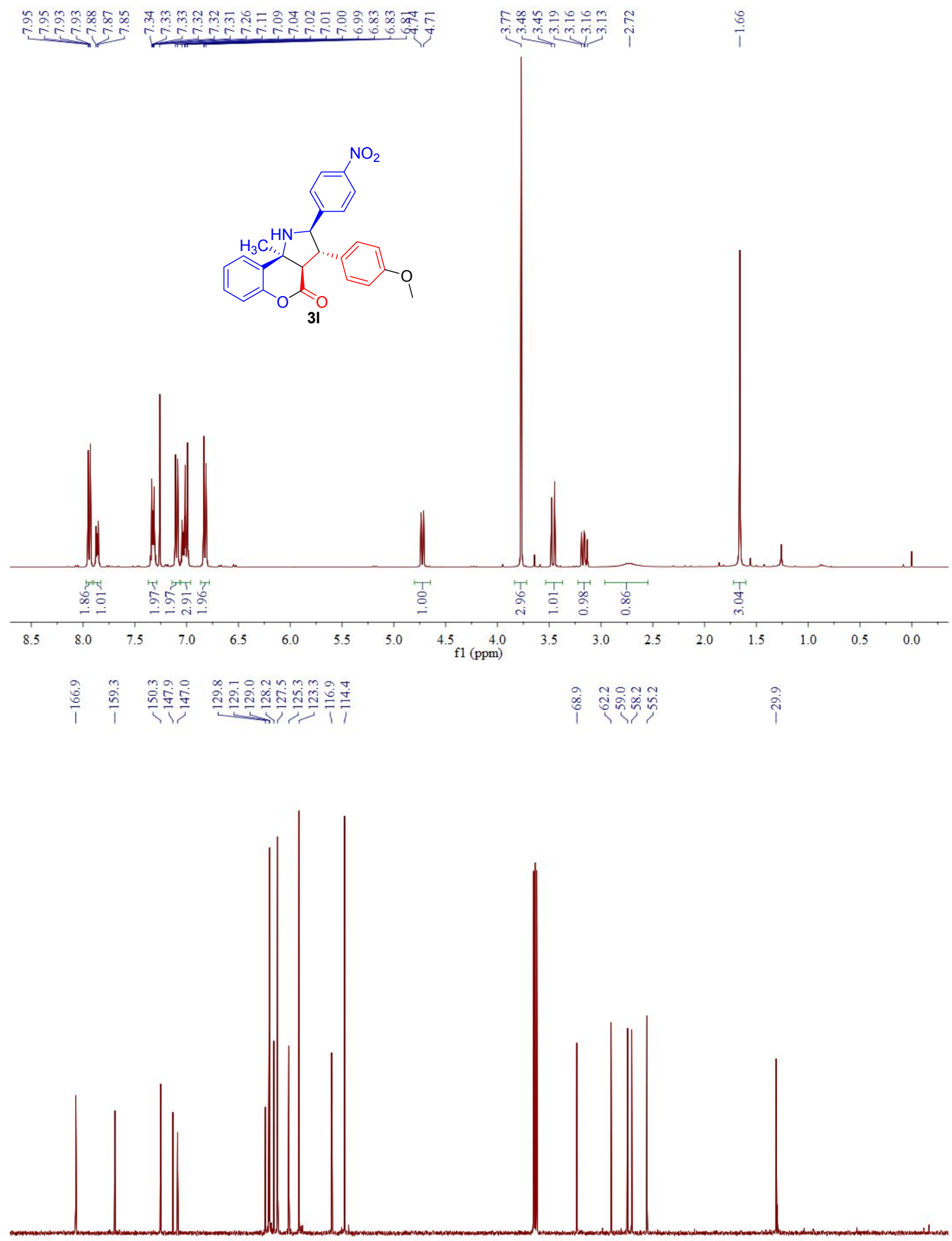

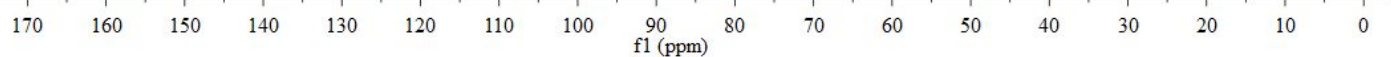




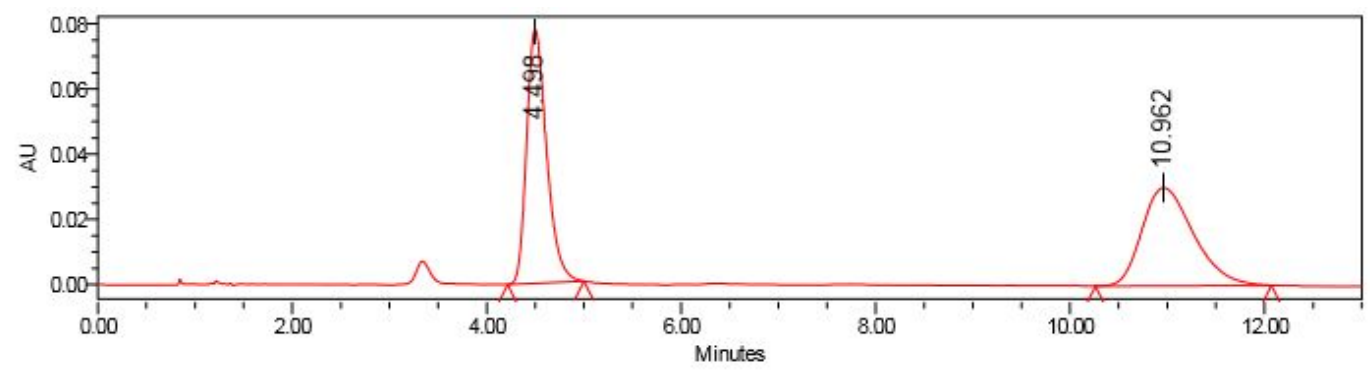

Peak Results

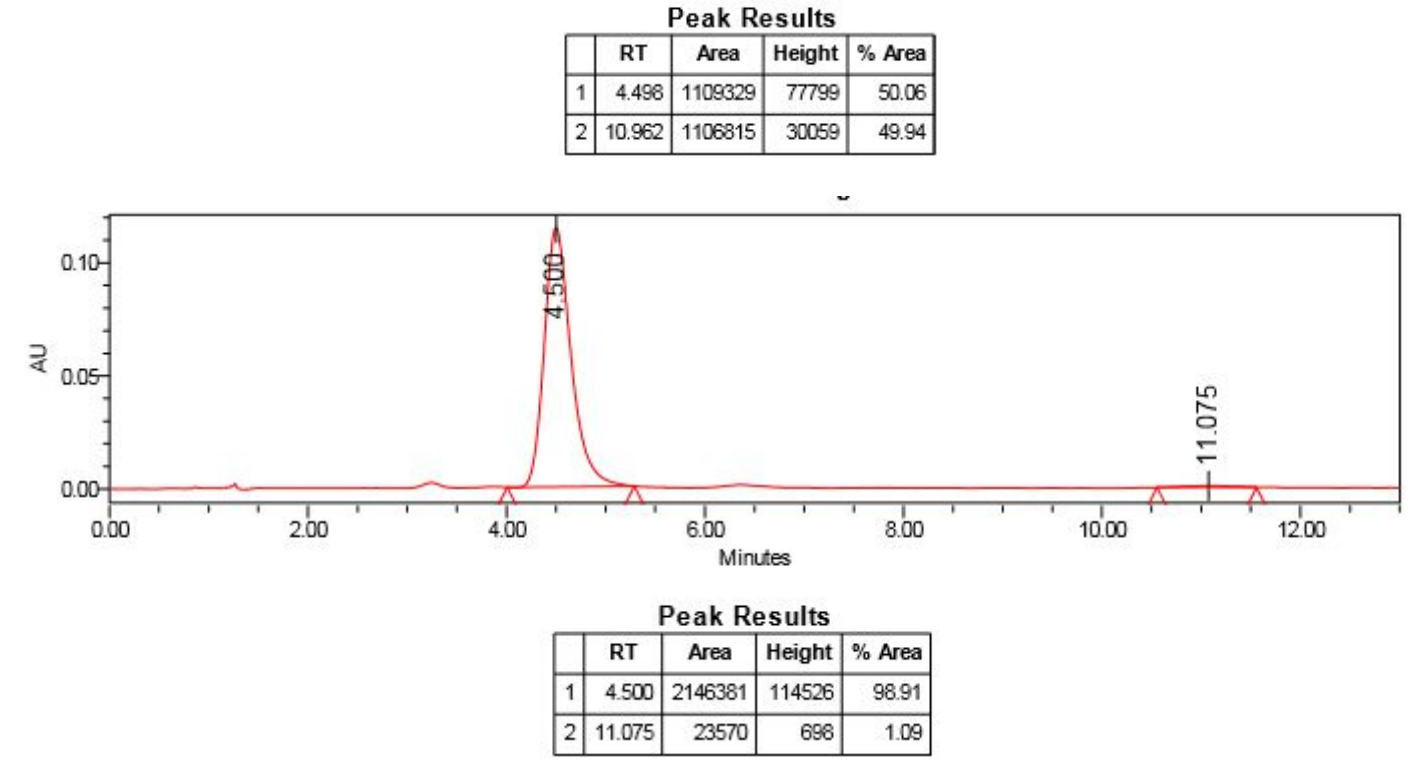


(2R,3S,3aR,9bS)-3-(4-(dimethylamino)phenyl)-9b-methyl-2-(4-nitrophenyl)-2,3,3 a,9b-tetrahydrochromeno[4,3-b]pyrrol-4(1H)-one $(3 \mathrm{~m})$ :

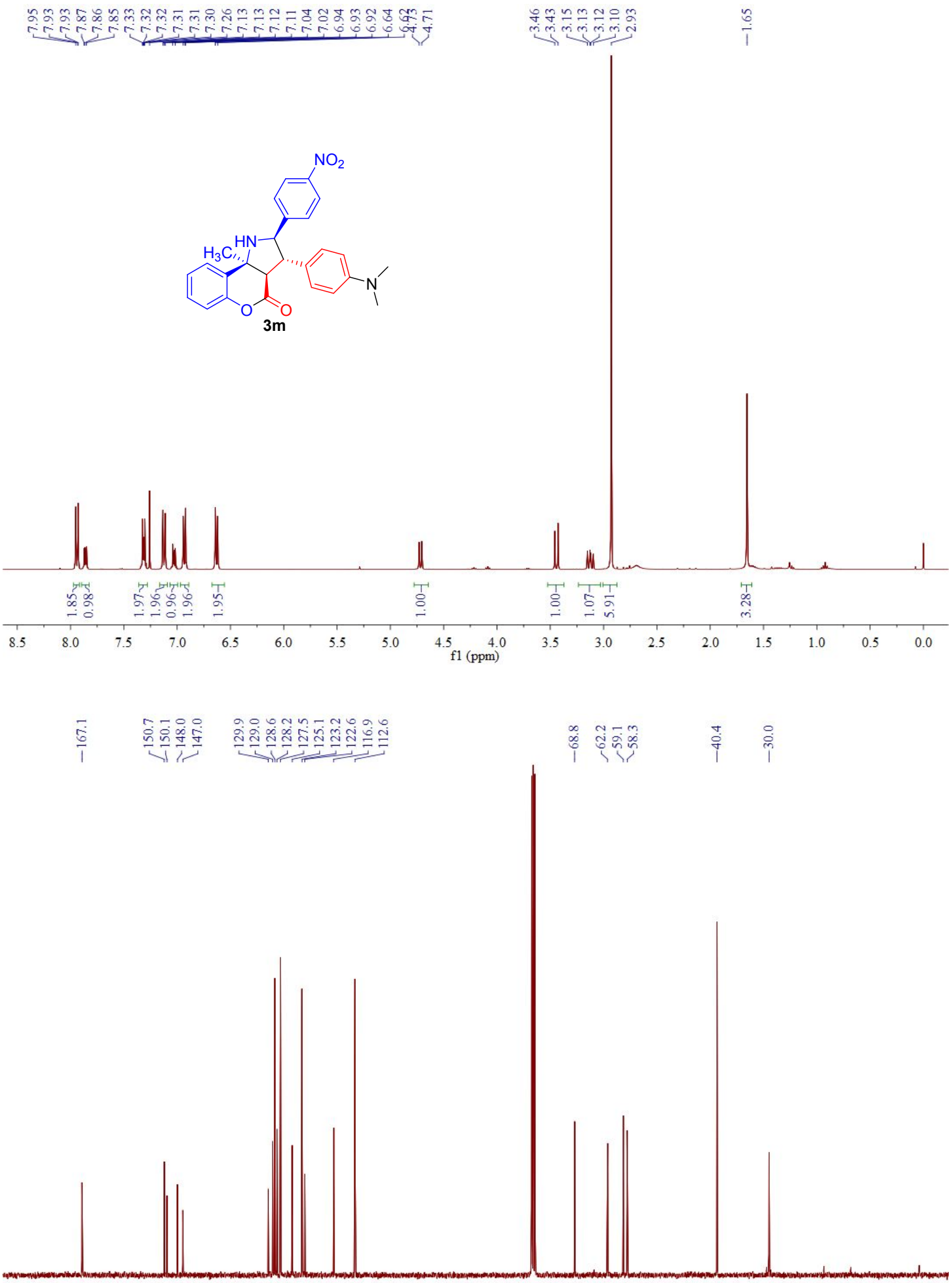

f1 (ppm) 


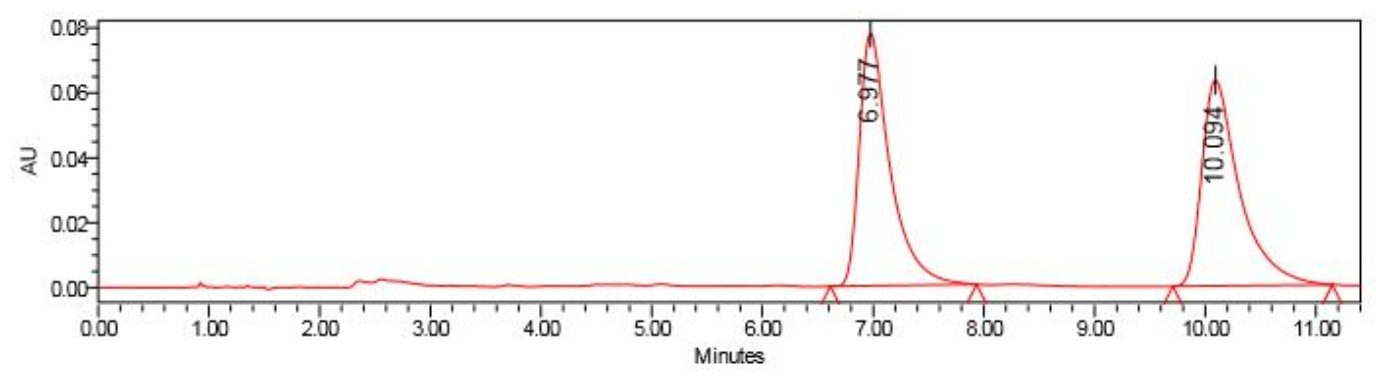

Peak Results

\begin{tabular}{|c|c|c|r|r|}
\hline & RT & Area & Height & $\%$ Area \\
\hline 1 & 6.977 & 1498187 & 77800 & 49.88 \\
\hline 2 & 10.094 & 1505256 & 63366 & 50.12 \\
\hline
\end{tabular}

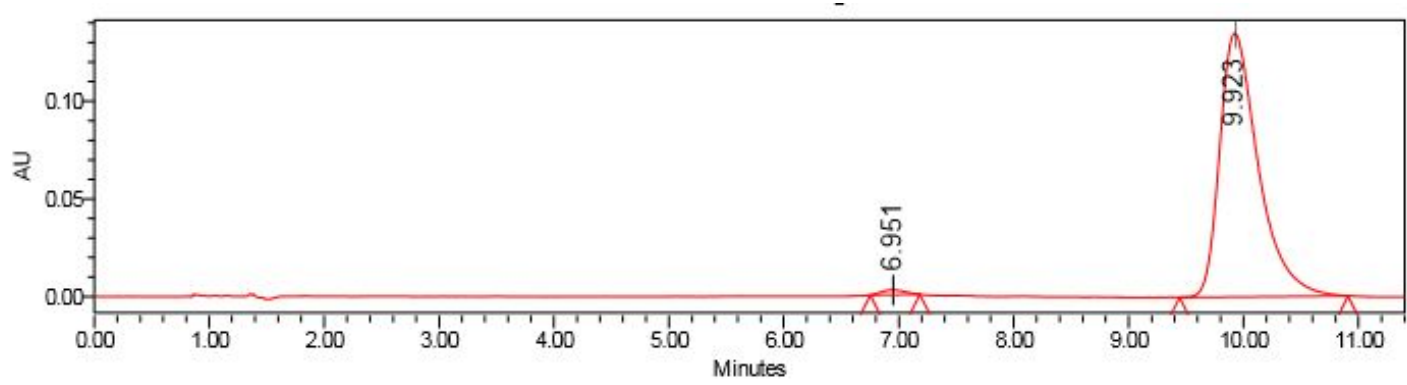

Peak Results

\begin{tabular}{|r|c|c|r|r|}
\hline & RT & Area & Height & $\%$ Area \\
\hline 1 & 6.951 & 33804 & 2423 & 1.06 \\
\hline 2 & 9.923 & 3143468 & 134681 & 98.94 \\
\hline
\end{tabular}


(2R,3R,3aR,9bS)-3-(furan-2-yl)-9b-methyl-2-(4-nitrophenyl)-2,3,3a,9b-tetrahydro chromeno[4,3-b]pyrrol-4(1H)-one $(3 n)$ :
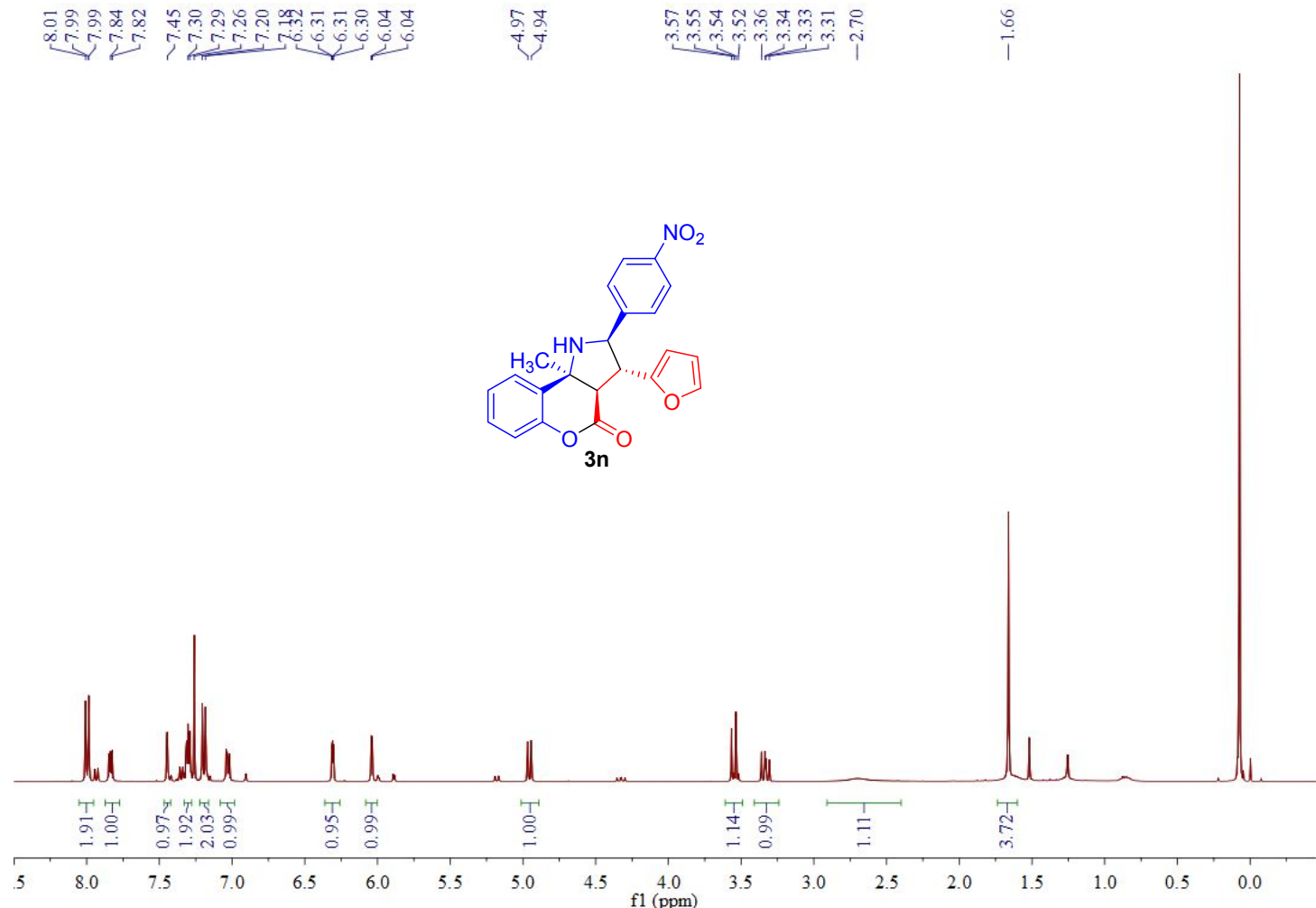

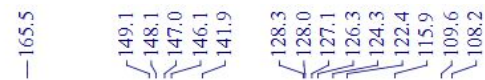

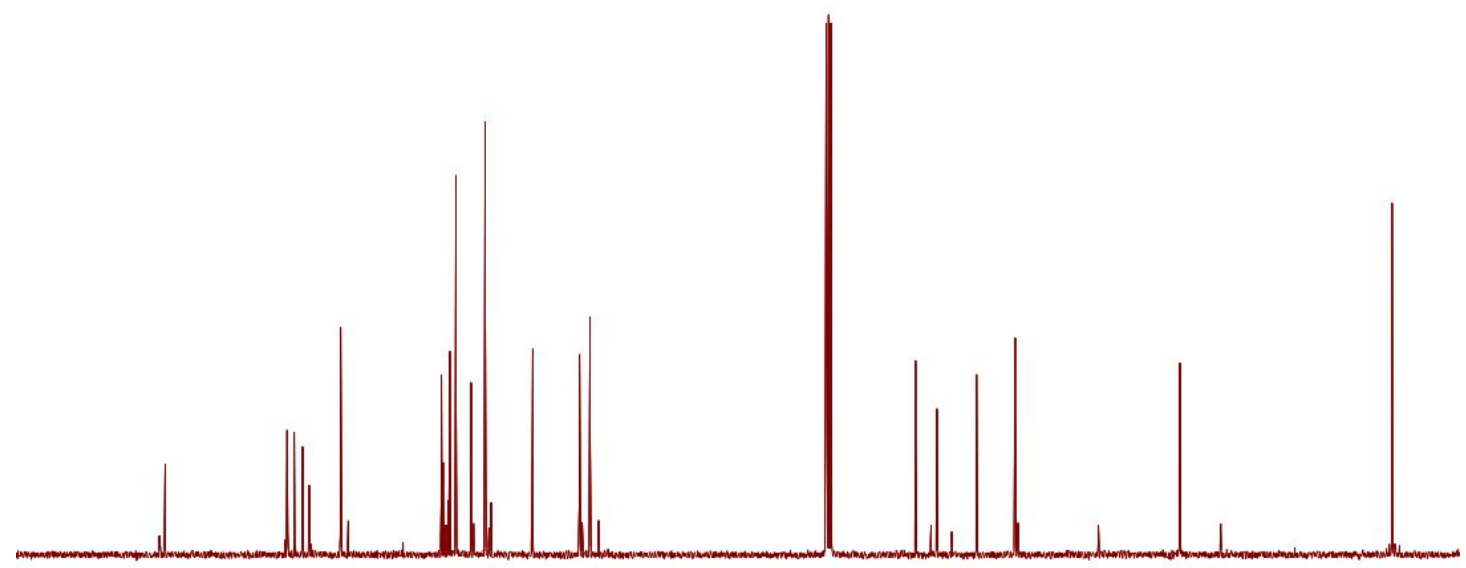

$\mathrm{fl}^{90}(\mathrm{ppm})$ 


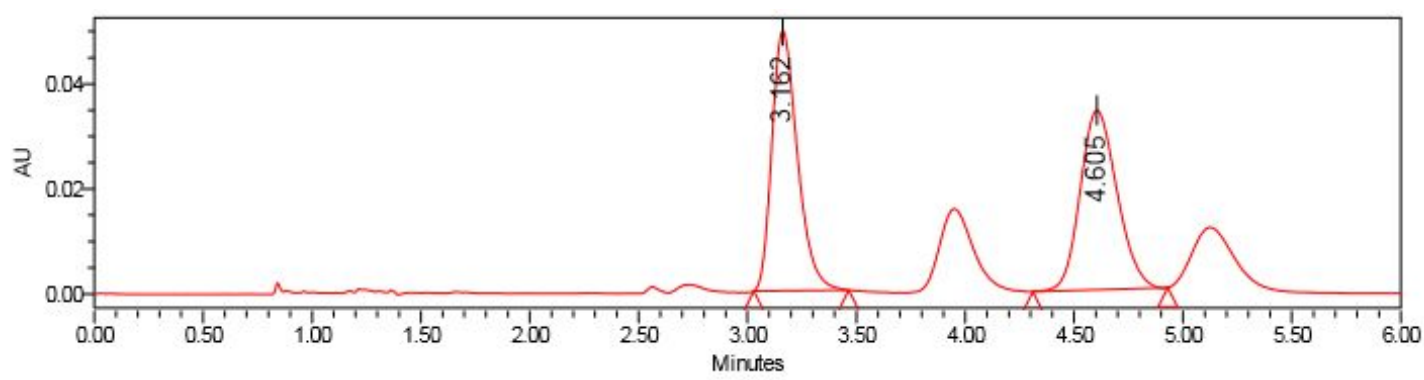

Peak Results

\begin{tabular}{|c|c|c|r|r|}
\hline & RT & Area & Height & $\%$ Area \\
\hline 1 & 3.162 & 403869 & 49483 & 50.15 \\
\hline 2 & 4.605 & 401375 & 34145 & 49.85 \\
\hline
\end{tabular}

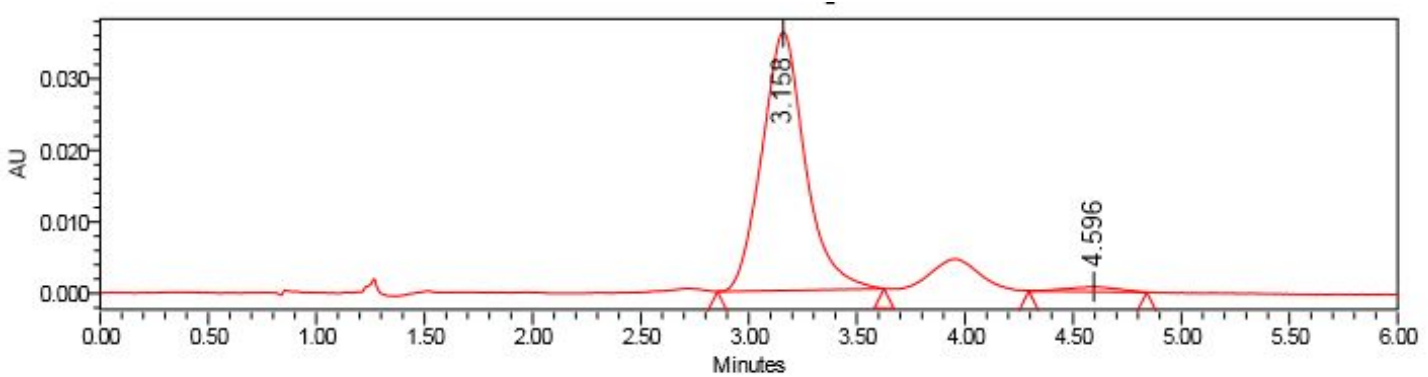

Peak Results

\begin{tabular}{|r|c|r|r|r|}
\hline & RT & Area & Height & $\%$ Area \\
\hline 1 & 3.158 & 503555 & 36116 & 97.95 \\
\hline 2 & 4.596 & 10535 & 669 & 2.05 \\
\hline
\end{tabular}


$(2 R, 3 R, 3 a R, 9 b S)-9 b-m e t h y l-2-(4-n i t r o p h e n y l)-3-(t h i o p h e n-2-y l)-2,3,3 a, 9 b-t e t r a h y$ drochromeno[4,3-b]pyrrol-4(1H)-one (3o):

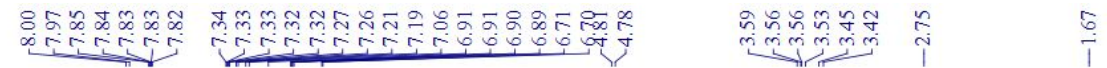
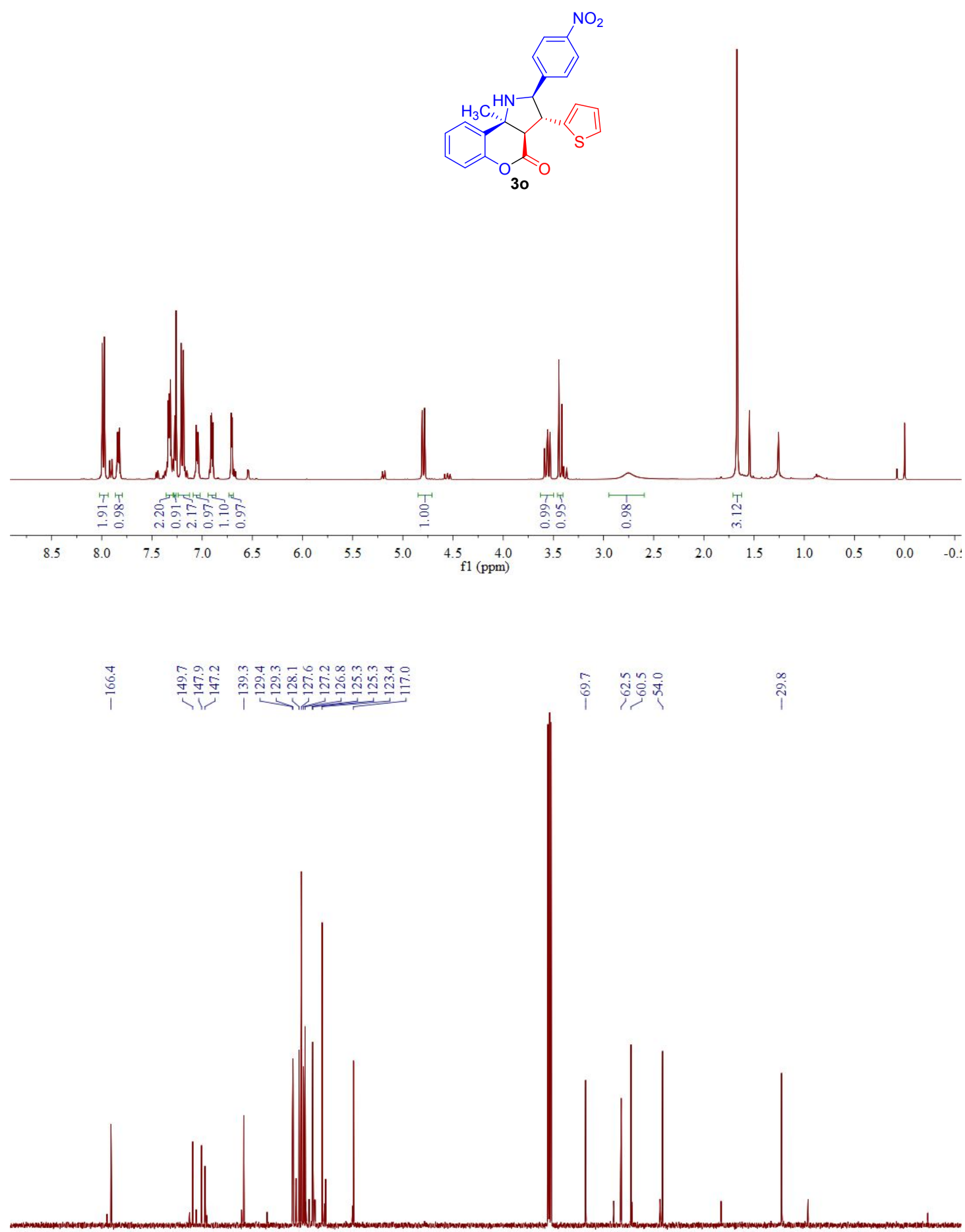

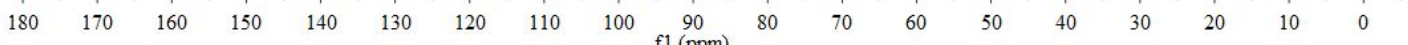




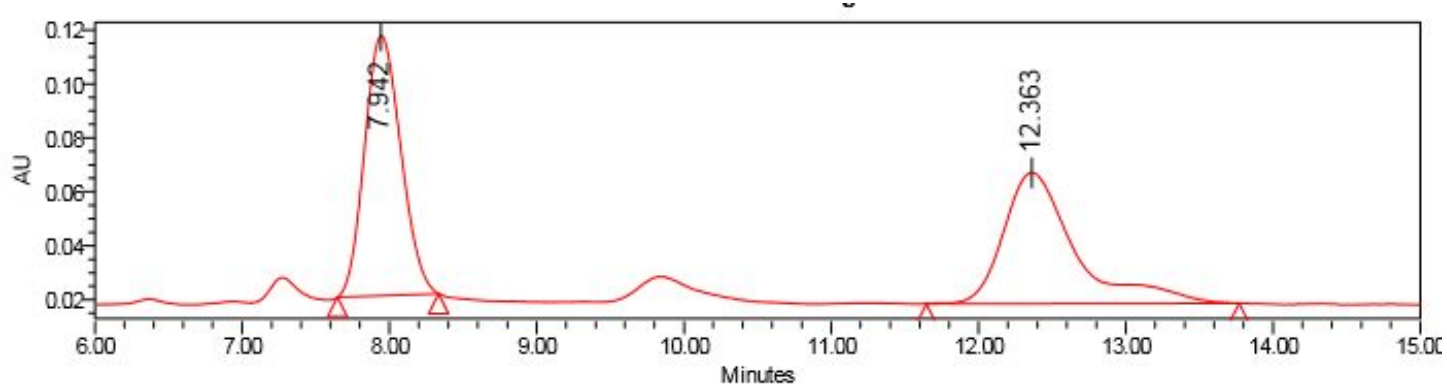

Peak Results

\begin{tabular}{|r|c|c|r|r|}
\hline & \multicolumn{1}{|c|}{ RT } & Area & Height & $\%$ Area \\
\hline 1 & 7.942 & 1652233 & 96386 & 50.33 \\
\hline 2 & 12.363 & 1630792 & 48568 & 49.67 \\
\hline
\end{tabular}

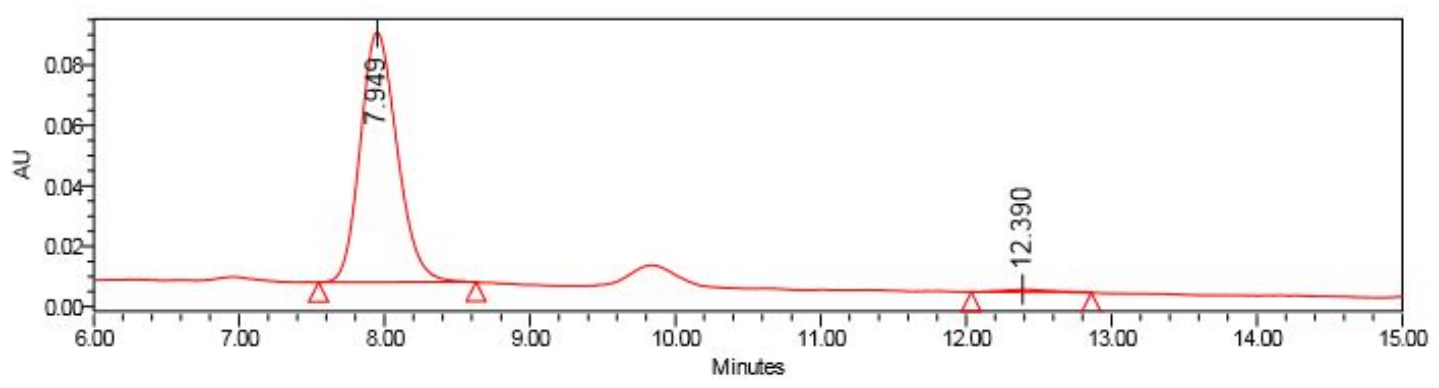

Peak Results

\begin{tabular}{|r|r|r|r|r|}
\hline & RT & \multicolumn{1}{c|}{ Area } & Height & $\%$ Area \\
\hline 1 & 7.949 & 1454794 & 82722 & 98.54 \\
\hline 2 & 12.390 & 21547 & 986 & 1.46 \\
\hline
\end{tabular}


(2R,3R,3aR,9bS)-3,9b-dimethyl-2-(4-nitrophenyl)-2,3,3a,9b-tetrahydrochromeno[ 4,3-b]pyrrol-4(1H)-one (3p):

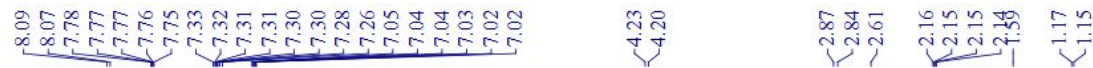

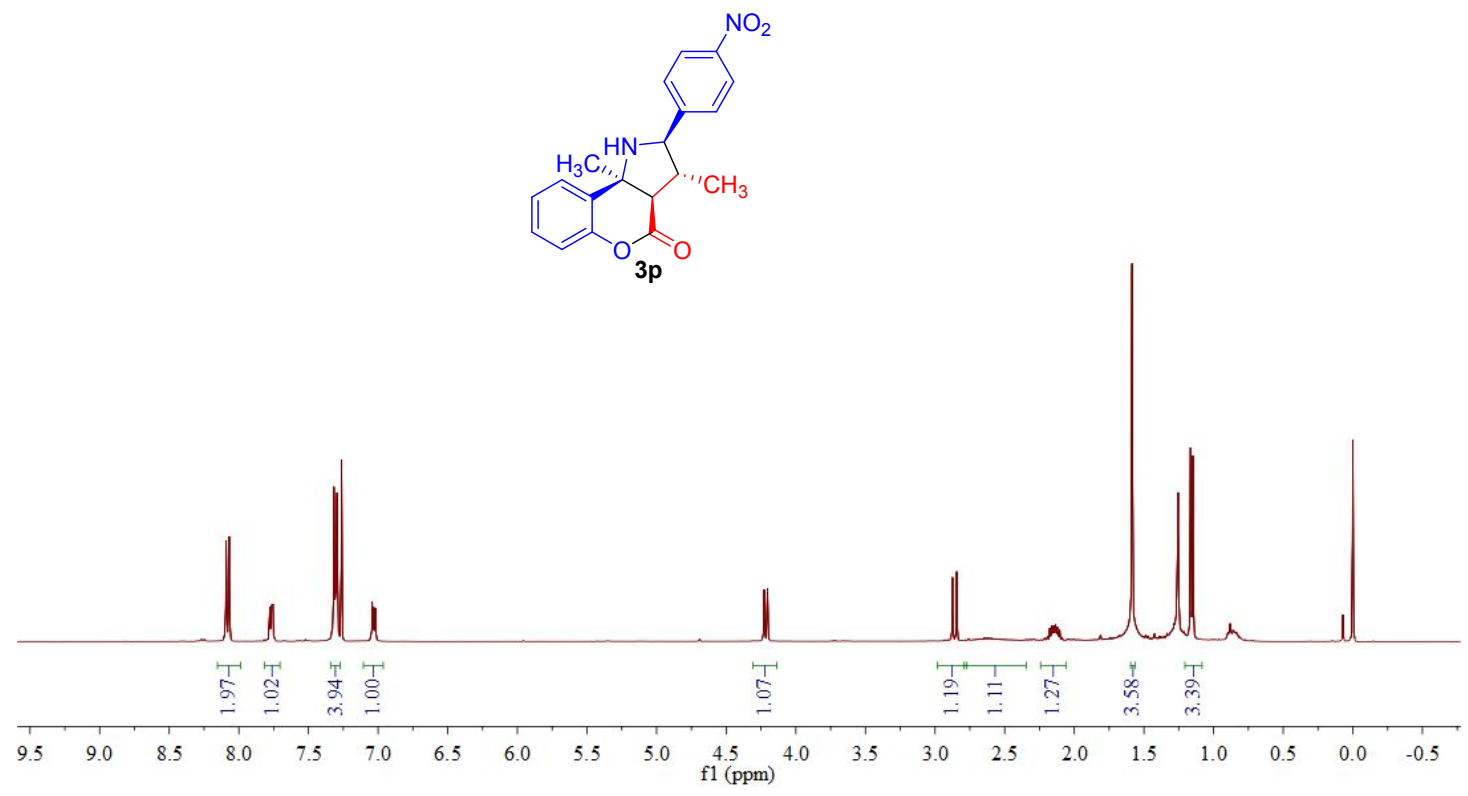

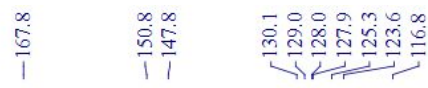

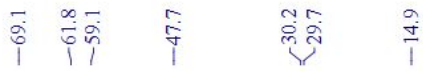

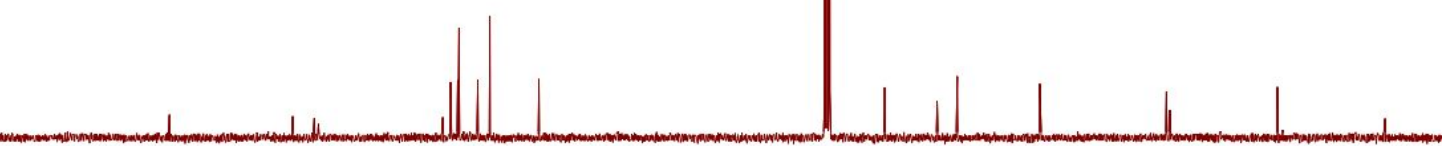

$\begin{array}{llllllllll}190 & 180 & 170 & 160 & 150 & 140 & 130 & 120 & 110 & 100 \\ \mathrm{f} 1(\mathrm{ppm}) & 90\end{array}$ 


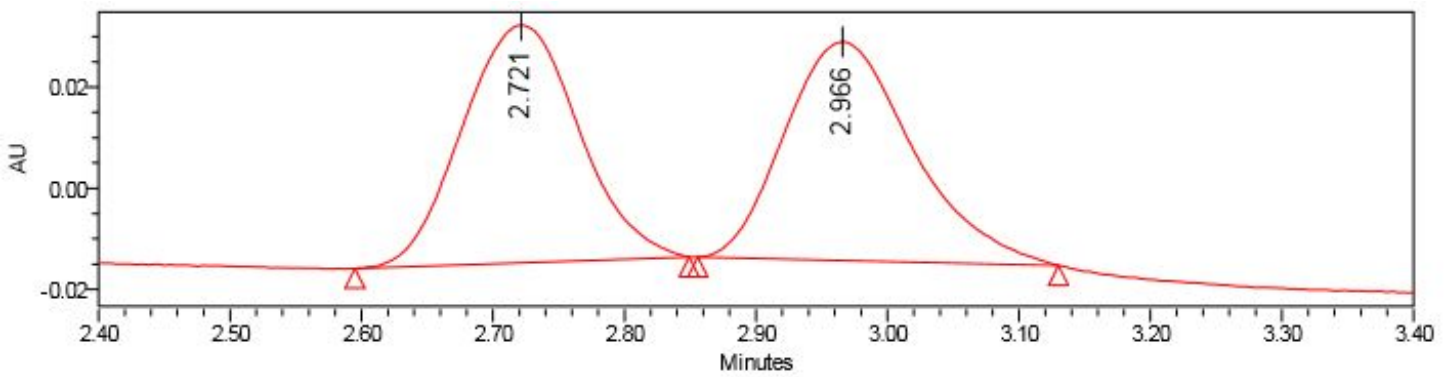

Peak Results

\begin{tabular}{|c|c|c|r|r|}
\hline & RT & Area & Height & $\%$ Area \\
\hline 1 & 2.721 & 269376 & 46978 & 49.78 \\
\hline 2 & 2.966 & 291913 & 43297 & 50.22 \\
\hline
\end{tabular}

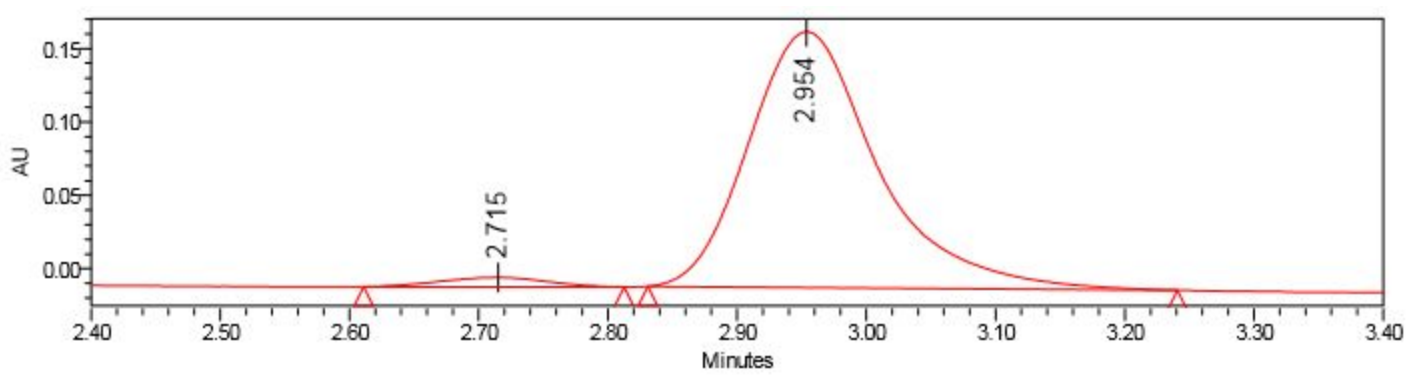

Peak Results

\begin{tabular}{|r|c|c|r|r|}
\hline & RT & Area & Height & $\%$ Area \\
\hline 1 & 2.715 & 36657 & 6450 & 2.94 \\
\hline 2 & 2.954 & 1209623 & 174407 & 97.06 \\
\hline
\end{tabular}


(2R,3S,3aR,9bS)-7-chloro-9b-methyl-2-(4-nitrophenyl)-3-phenyl-2,3,3a,9b-tetrah ydrochromeno[4,3-b]pyrrol-4(1H)-one (4a):

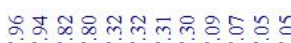

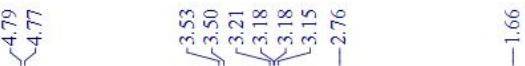

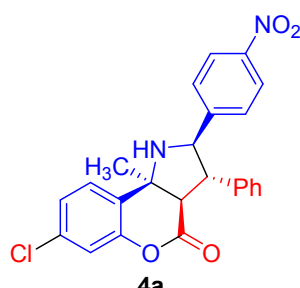

$4 a$
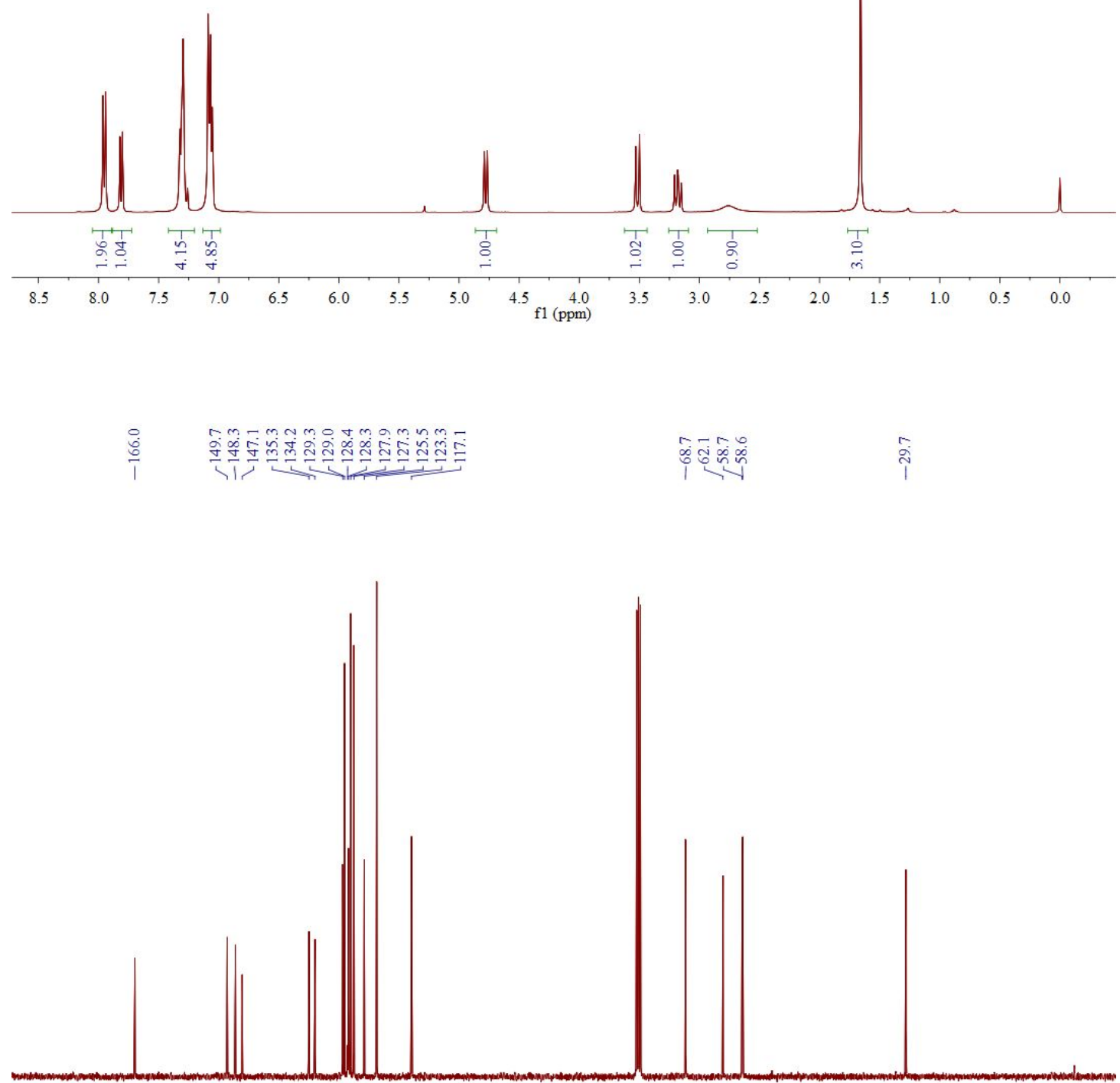

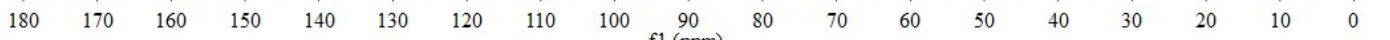




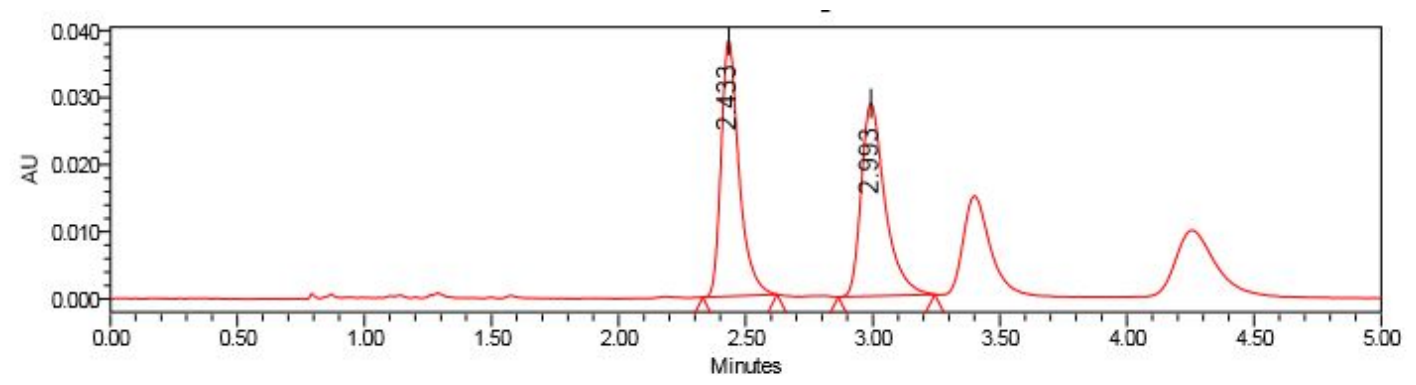

Peak Results
\begin{tabular}{|c|c|c|r|r|}
\hline & RT & Area & Height & $\%$ Area \\
\hline 1 & 2.433 & 187036 & 38167 & 50.00 \\
\hline 2 & 2.993 & 187015 & 28710 & 50.00 \\
\hline
\end{tabular}

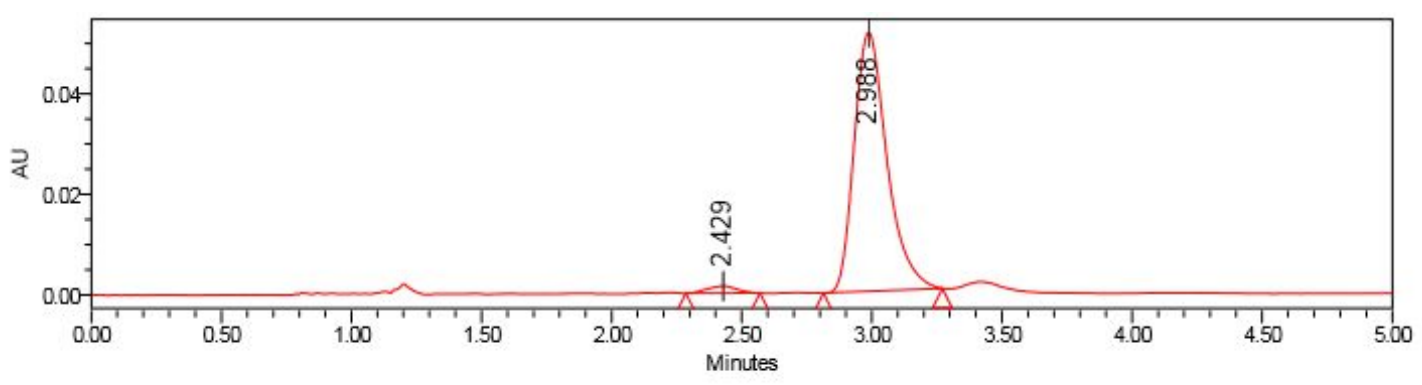

Peak Results

\begin{tabular}{|r|c|r|r|r|}
\hline & RT & Area & Height & $\%$ Area \\
\hline 1 & 2.429 & 10922 & 1407 & 237 \\
\hline 2 & 2.988 & 450848 & 51429 & 97.63 \\
\hline
\end{tabular}


(2R,3S,3aR,9bS)-7-bromo-9b-methyl-2-(4-nitrophenyl)-3-phenyl-2,3,3a,9b-tetrah ydrochromeno[4,3-b]pyrrol-4(1H)-one $(4 \mathrm{~b})$ :

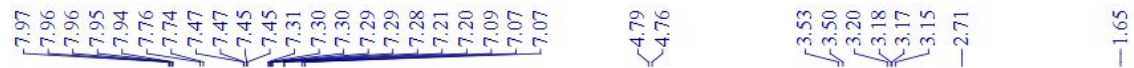
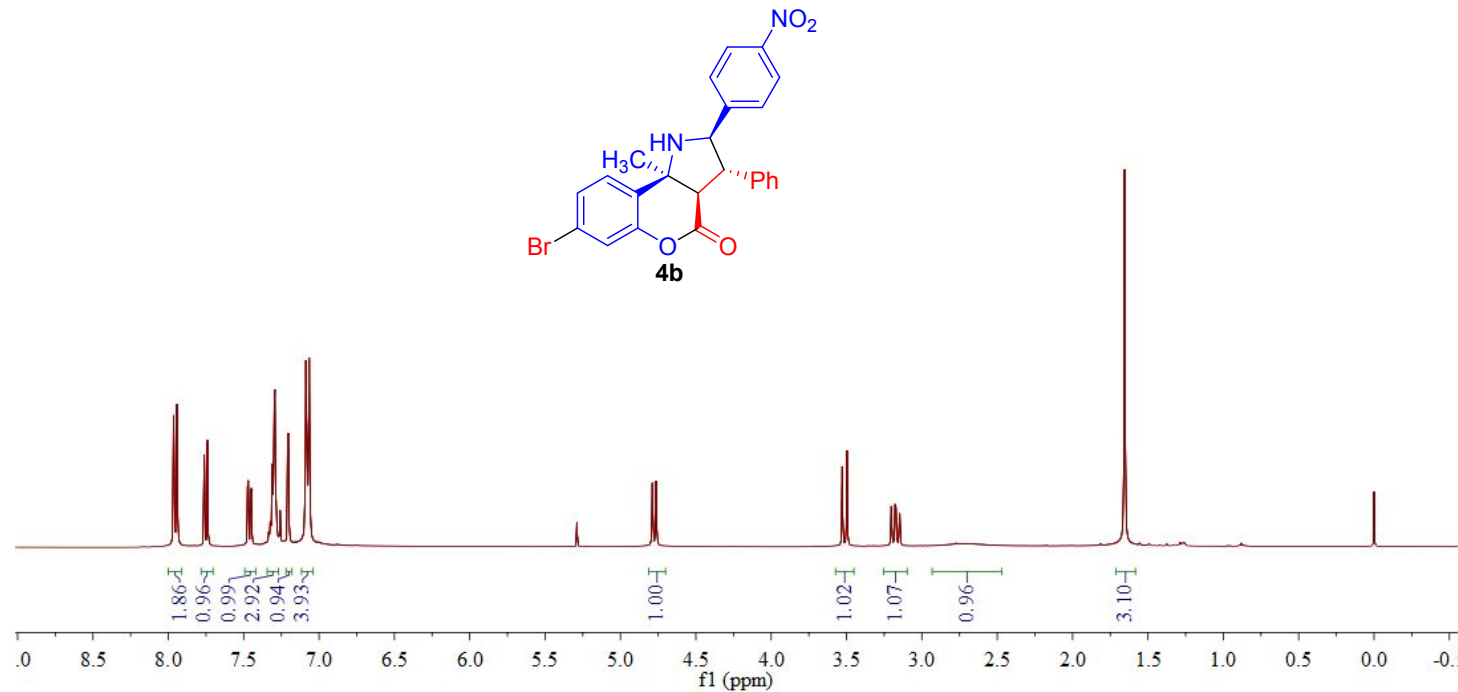

lo

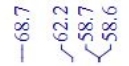

ì

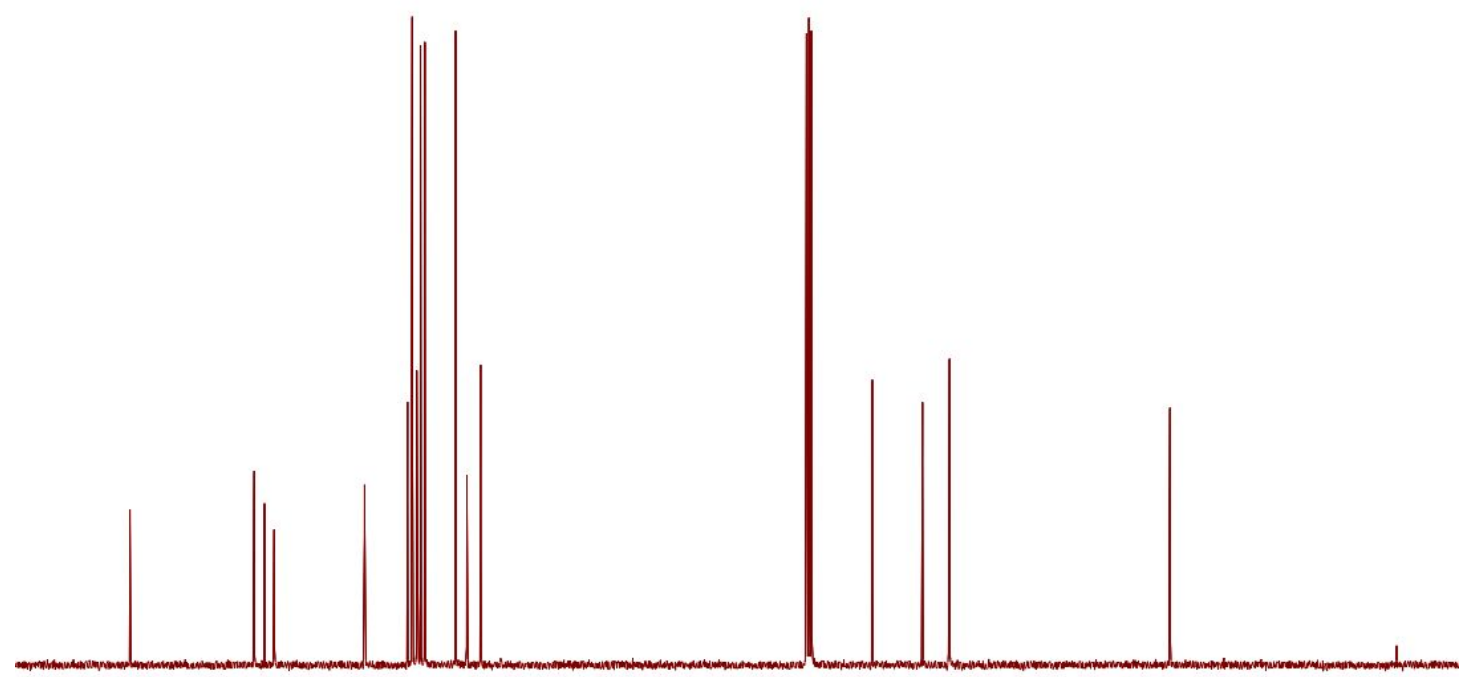

80

$\begin{array}{lllllllll}170 & 160 & 150 & 140 & 130 & 120 & 110 & 100 & 90 \\ \mathrm{f1}(\mathrm{ppm}) & 80\end{array}$ 


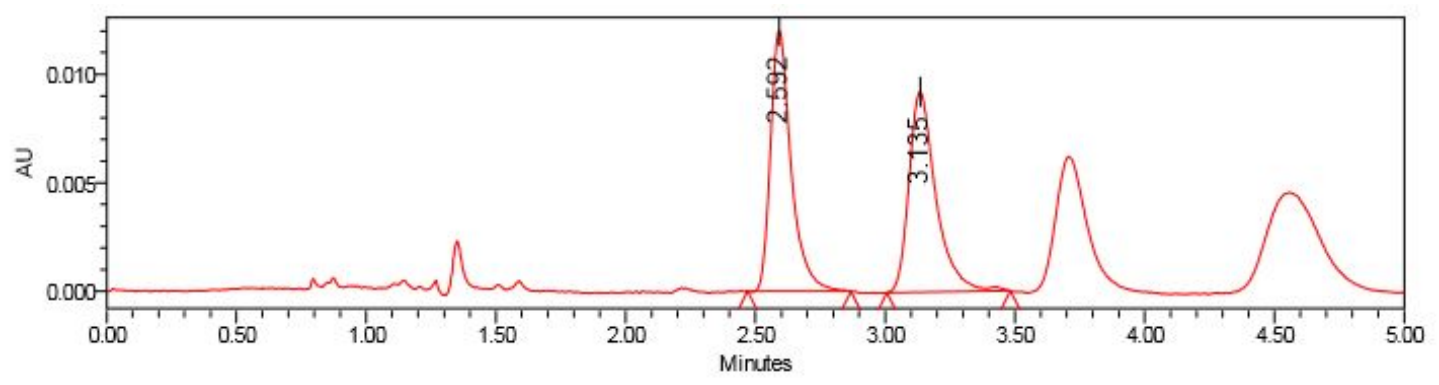

Peak Results

\begin{tabular}{|r|c|c|r|r|}
\hline & RT & Area & Height & $\%$ Area \\
\hline 1 & 2.592 & 66372 & 12006 & 49.87 \\
\hline 2 & 3.135 & 66718 & 9231 & 50.13 \\
\hline
\end{tabular}

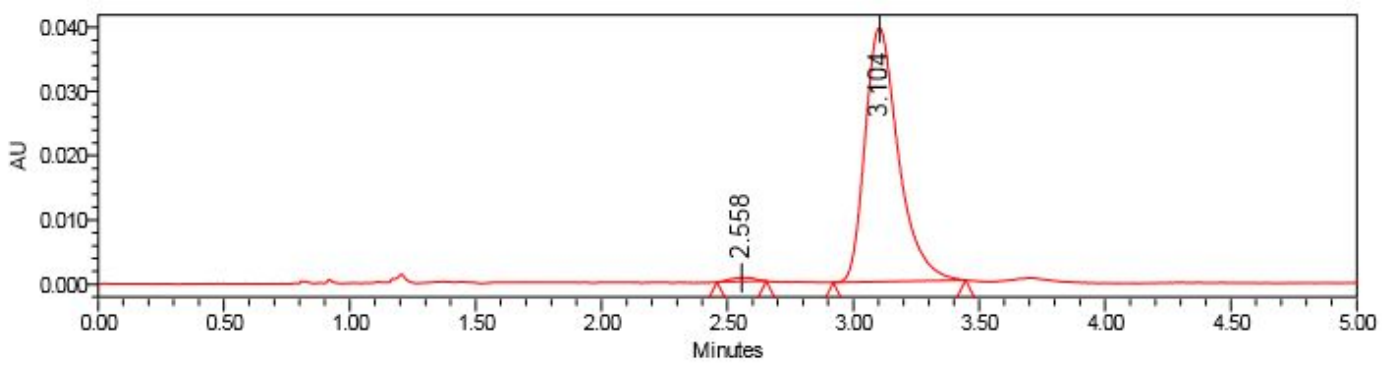

Peak Results

\begin{tabular}{|r|c|r|r|r|}
\hline & RT & Area & Height & $\%$ Area \\
\hline 1 & 2.558 & 3913 & 599 & 1.06 \\
\hline 2 & 3.104 & 364261 & 39486 & 98.94 \\
\hline
\end{tabular}


(2R,3S,3aR,9bS)-7,9b-dimethyl-2-(4-nitrophenyl)-3-phenyl-2,3,3a,9b-tetrahydroc hromeno[4,3-b]pyrrol-4(1H)-one $(4 c)$ :

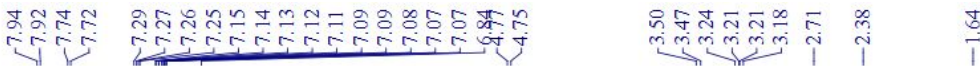
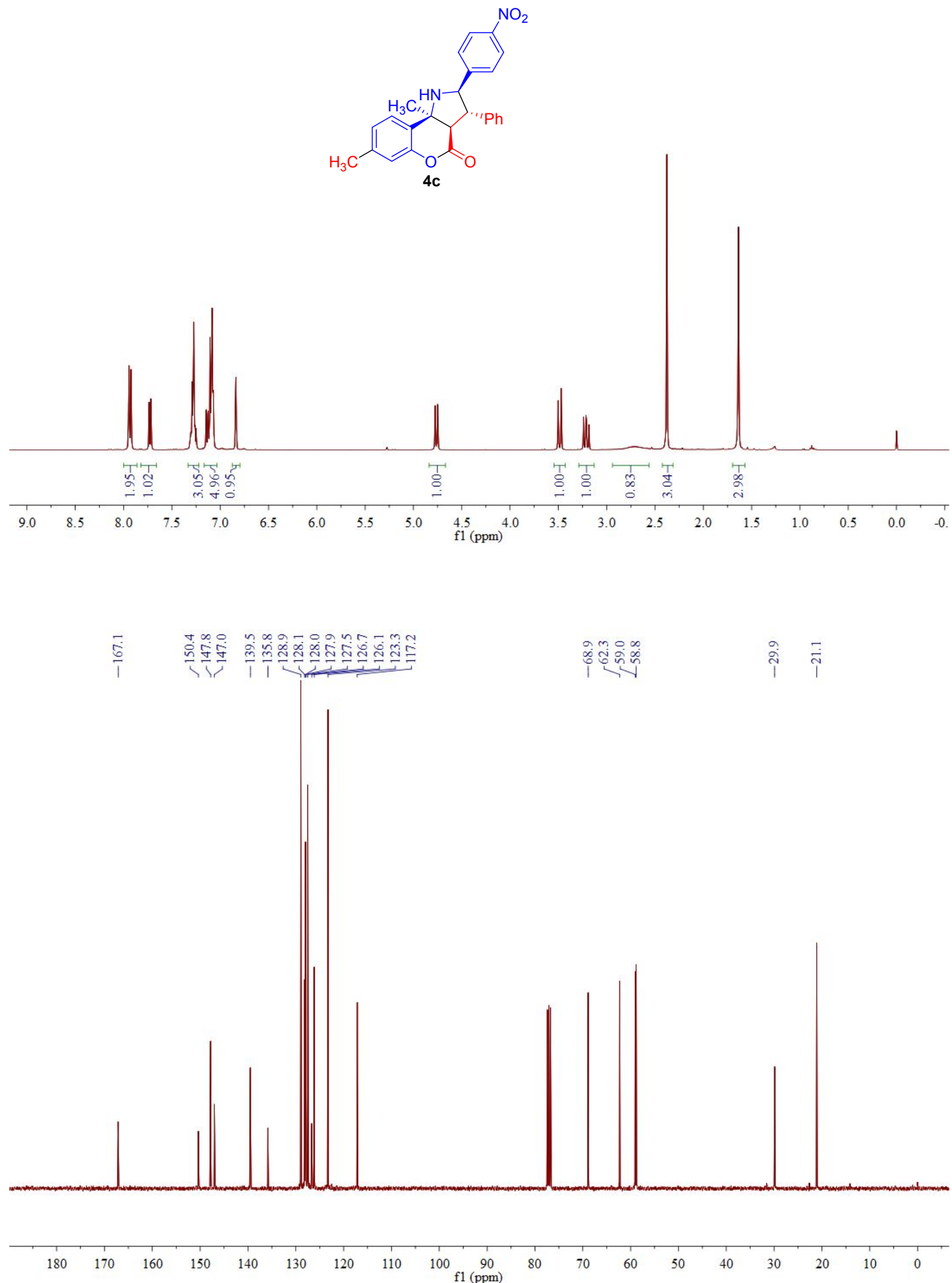


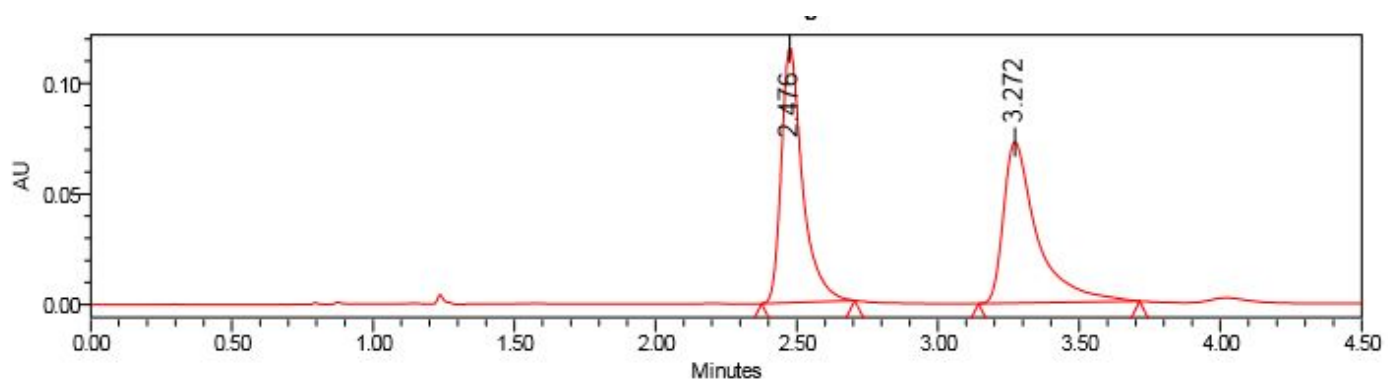

Peak Results
\begin{tabular}{|c|c|c|c|r|}
\hline & RT & Area & Height & $\%$ Area \\
\hline 1 & 2.476 & 602956 & 115148 & 50.08 \\
\hline 2 & 3.272 & 601047 & 72791 & 49.92 \\
\hline
\end{tabular}

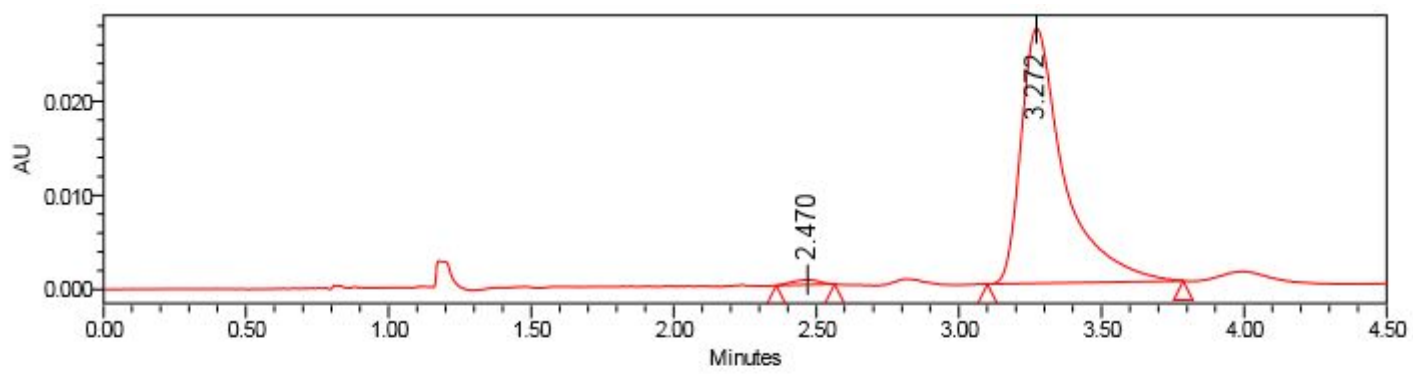

Peak Results

\begin{tabular}{|r|c|r|r|r|}
\hline & RT & Area & Height & $\%$ Area \\
\hline 1 & 2.470 & 3511 & 554 & 1.22 \\
\hline 2 & 3.272 & 284154 & 27107 & 98.78 \\
\hline
\end{tabular}


(2R,3S,3aR,9bS)-7-methoxy-9b-methyl-2-(4-nitrophenyl)-3-phenyl-2,3,3a,9b-tetr ahydrochromeno $[4,3-b]$ pyrrol-4(1H)-one $(4 \mathrm{~d})$ :
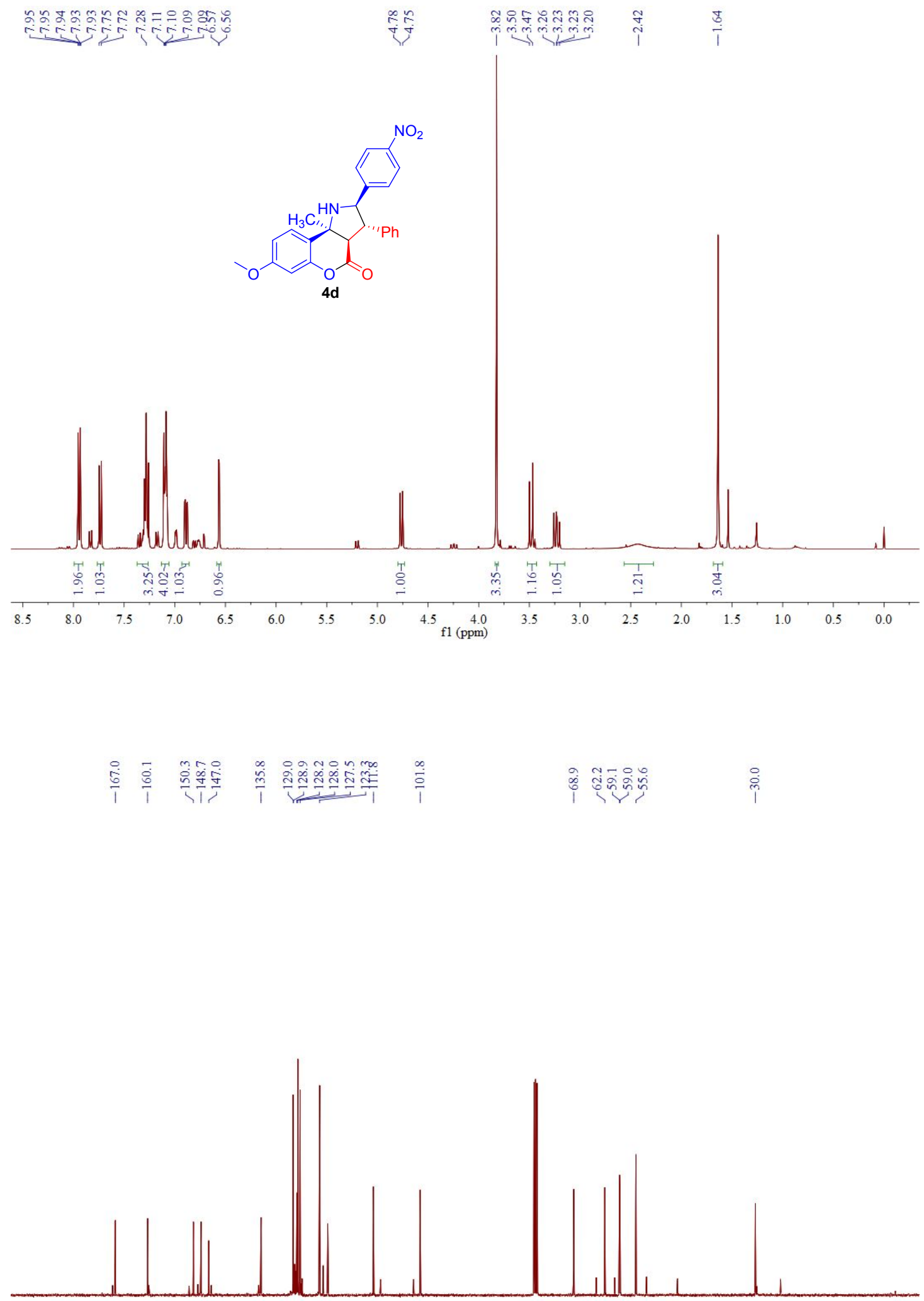

$\begin{array}{lllllllll}180 & 170 & 160 & 150 & 140 & 130 & 120 & 110 & 100 \\ \mathrm{f} 1(\mathrm{ppm})\end{array}$ 


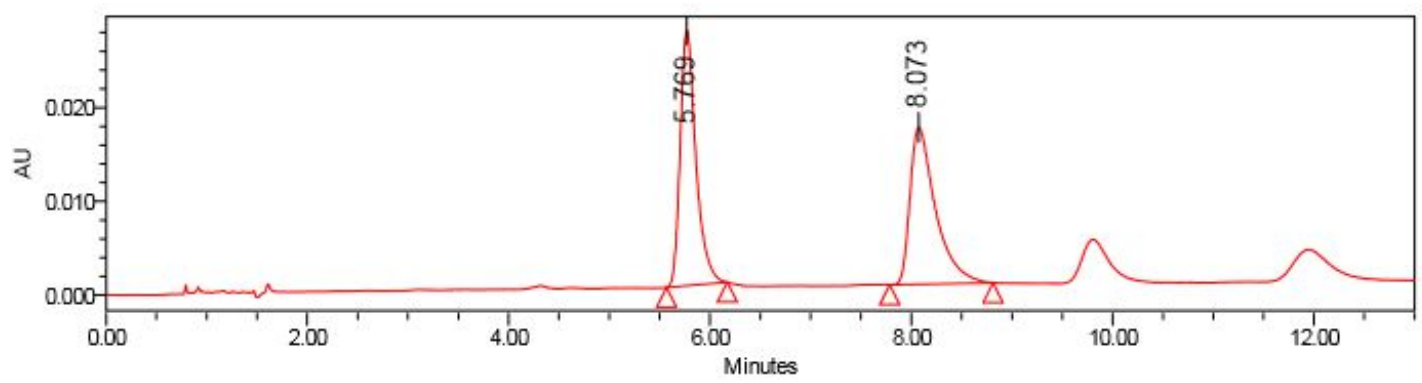

Peak Results

\begin{tabular}{|c|c|c|r|r|}
\hline & RT & Area & Height & $\%$ Area \\
\hline 1 & 5.769 & 295002 & 27263 & 50.07 \\
\hline 2 & 8.073 & 294204 & 16715 & 49.93 \\
\hline
\end{tabular}

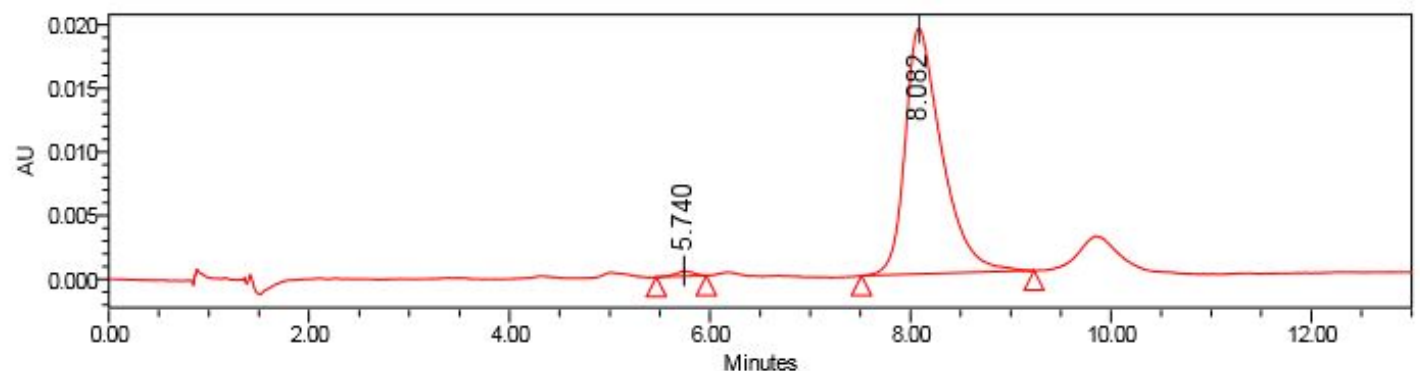

Peak Results

\begin{tabular}{|l|c|r|r|r|}
\hline & RT & Area & Height & \% Area \\
\hline 1 & 5.740 & 5138 & 433 & 1.03 \\
\hline 2 & 8.082 & 493710 & 19339 & 98.97 \\
\hline
\end{tabular}


(2R,3S,3aR,9bS)-8-fluoro-9b-methyl-2-(4-nitrophenyl)-3-phenyl-2,3,3a,9b-tetrah ydrochromeno[4,3-b]pyrrol-4(1H)-one (4e):

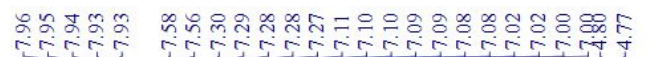

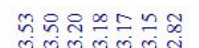
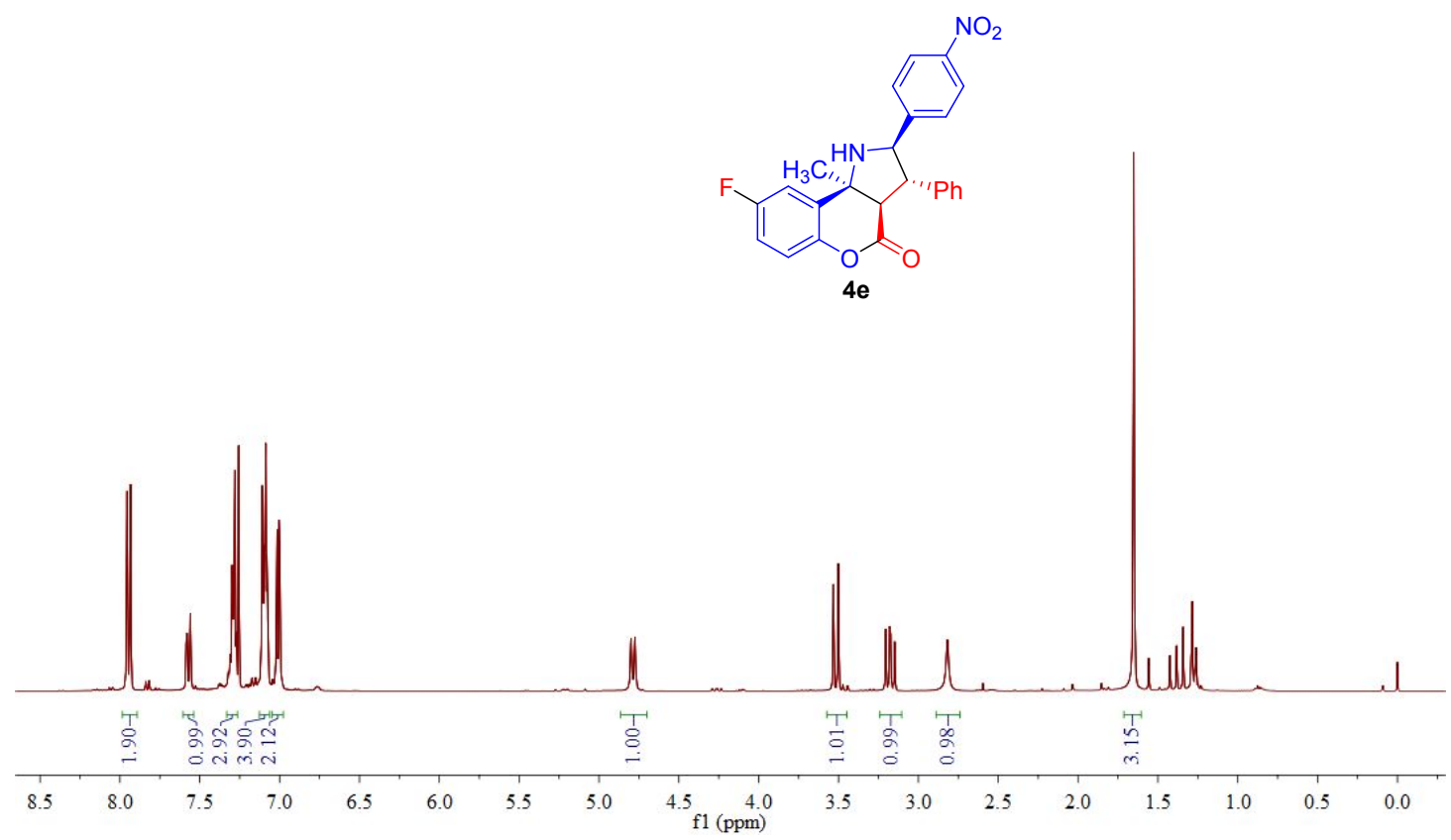

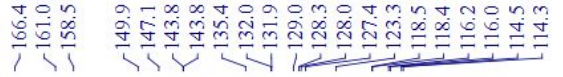

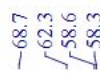

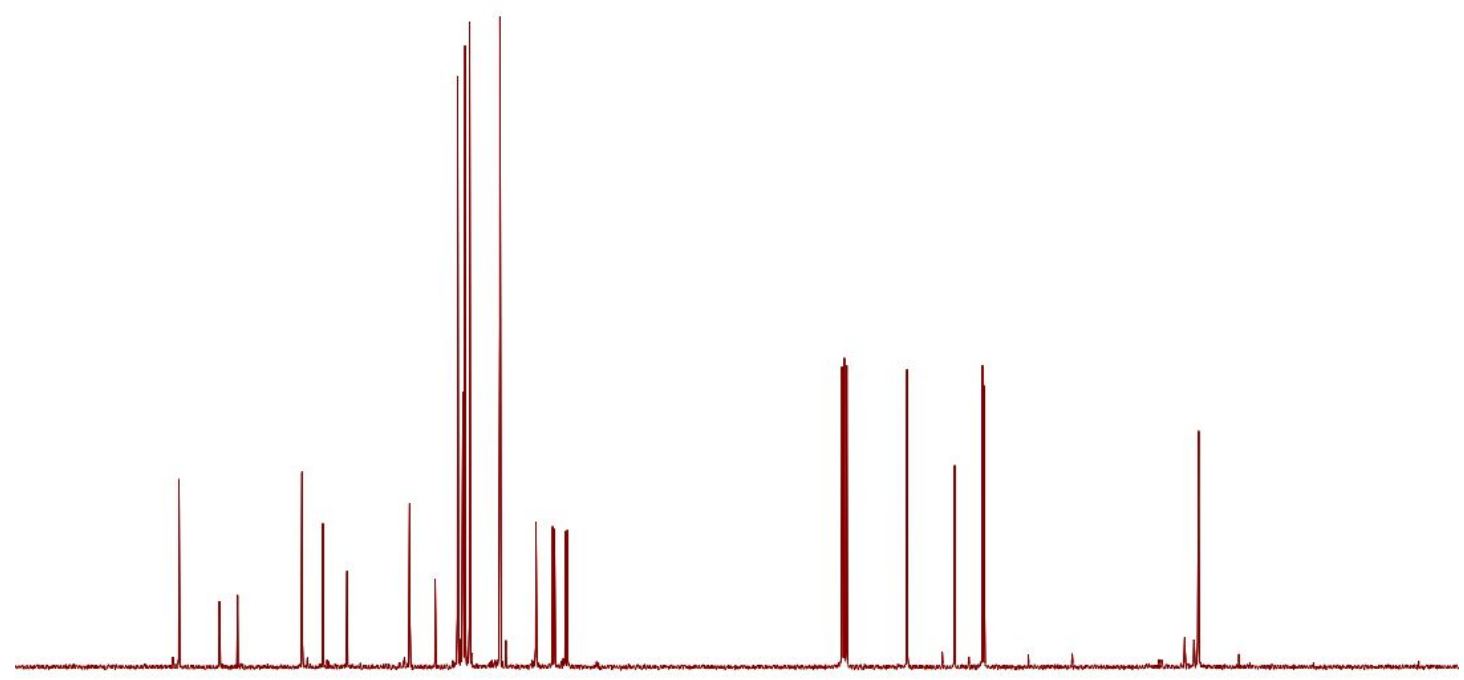

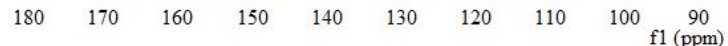




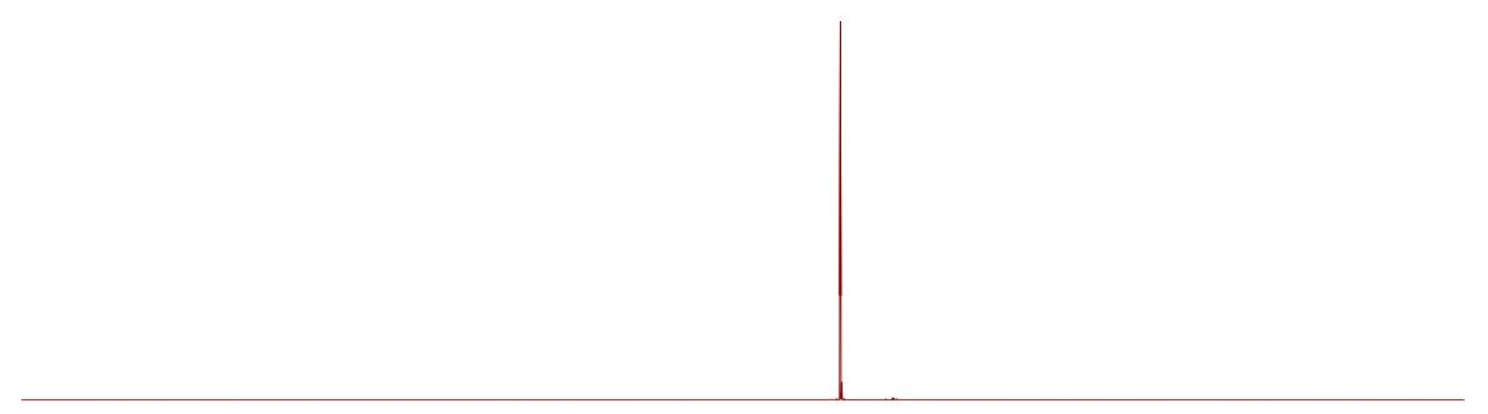

\begin{tabular}{llllllllllllllllllll}
10 & 10 & 0 & -10 & -20 & -30 & -40 & -50 & -60 & -70 & -80 & -90 & -110 & -130 & -150 & -170 & -190 & -210 \\
\hline
\end{tabular}

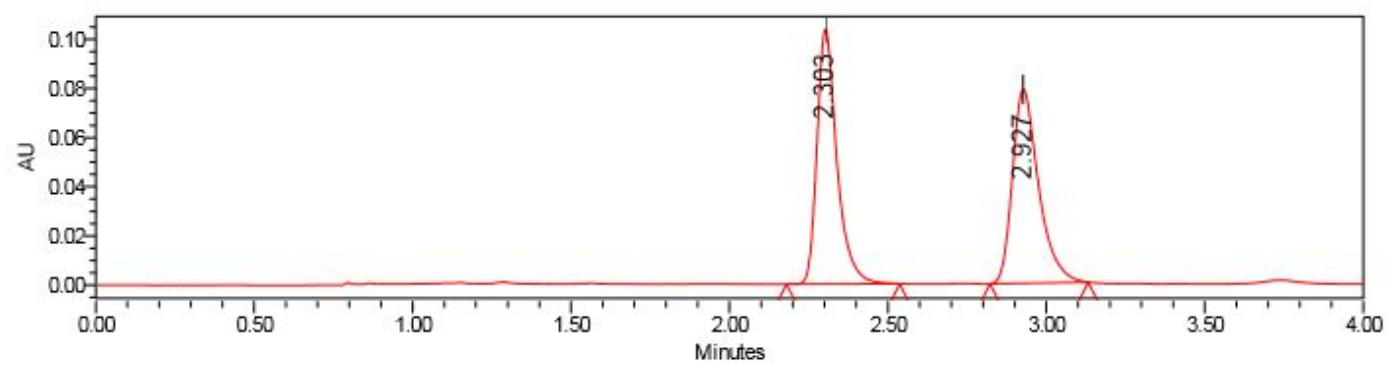

Peak Results

\begin{tabular}{|c|c|c|c|r|}
\hline & RT & Area & Height & $\%$ Area \\
\hline 1 & 2.303 & 452080 & 103763 & 49.91 \\
\hline 2 & 2.927 & 453637 & 78894 & 50.09 \\
\hline
\end{tabular}

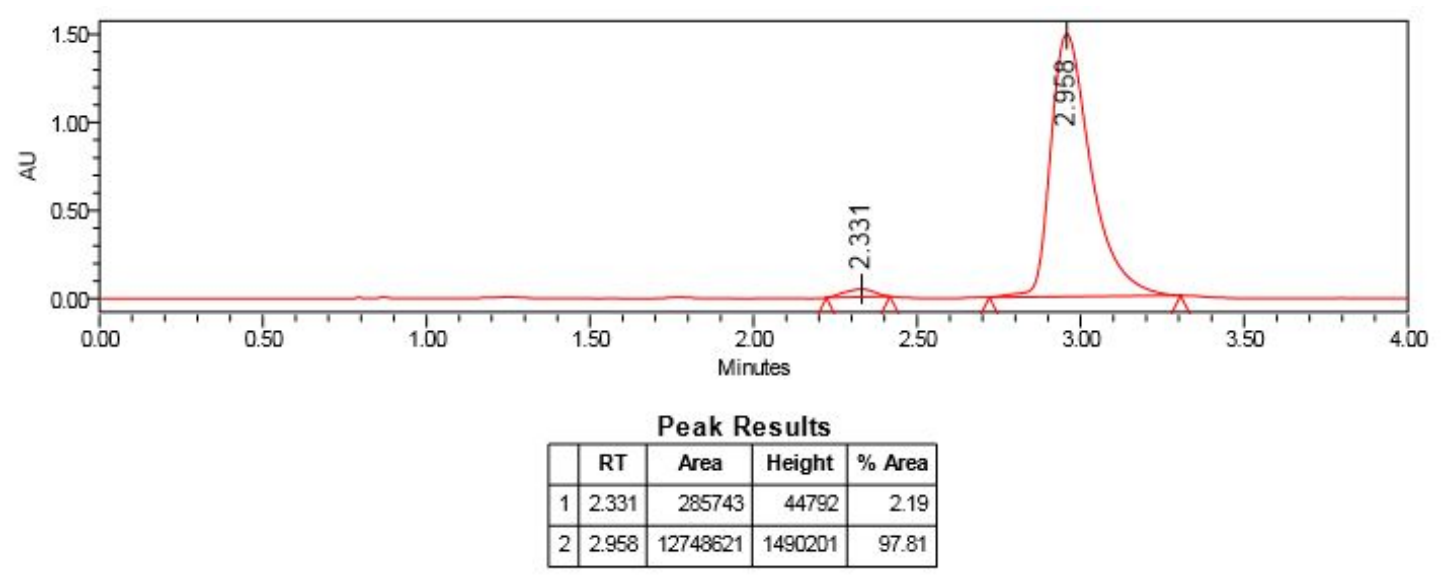


(2R,3S,3aR,9bS)-8-chloro-9b-methyl-2-(4-nitrophenyl)-3-phenyl-2,3,3a,9b-tetrah ydrochromeno[4,3-b]pyrrol-4(1H)-one (4f):
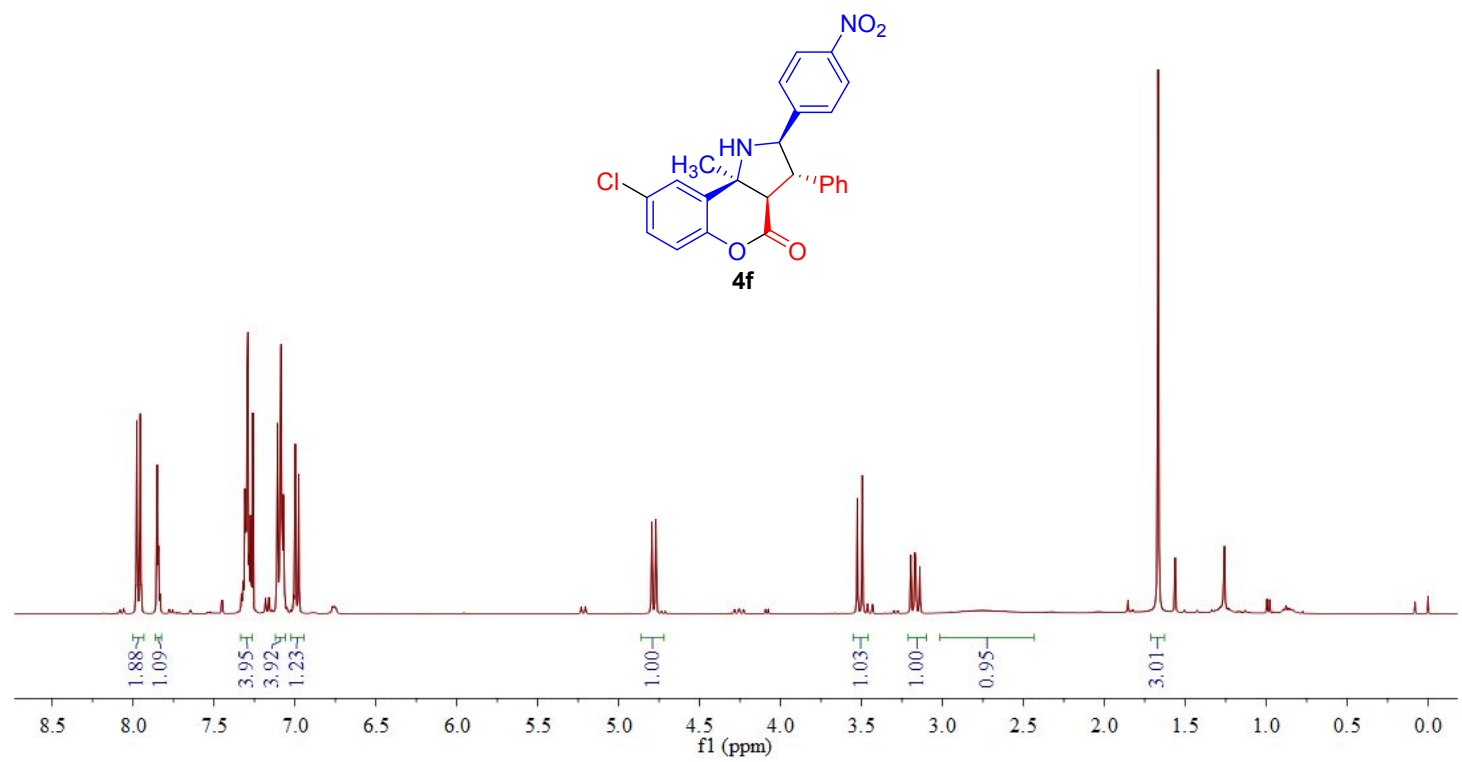

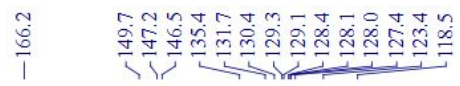

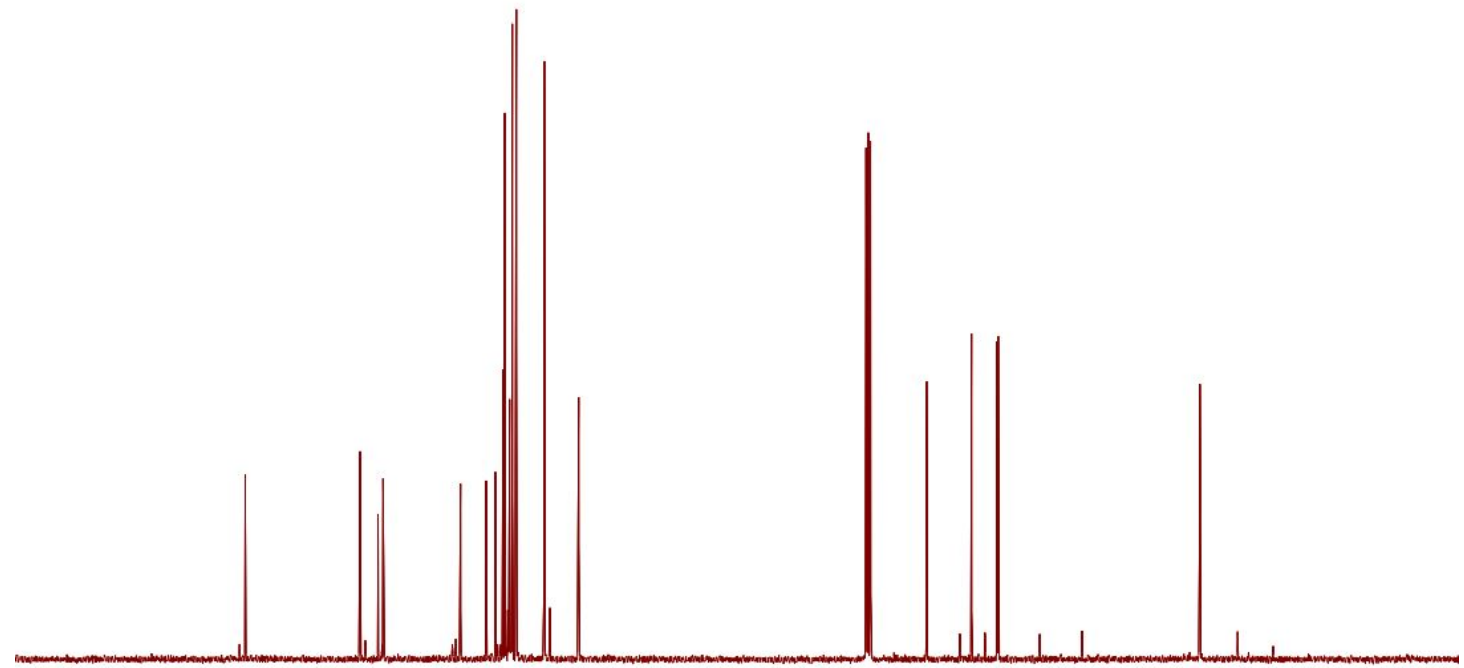

$\begin{array}{llllllllll}190 & 180 & 170 & 160 & 150 & 140 & 130 & 120 & 110 & \begin{array}{l}100 \\ \mathrm{f} 1(\mathrm{ppm})\end{array}\end{array}$ 

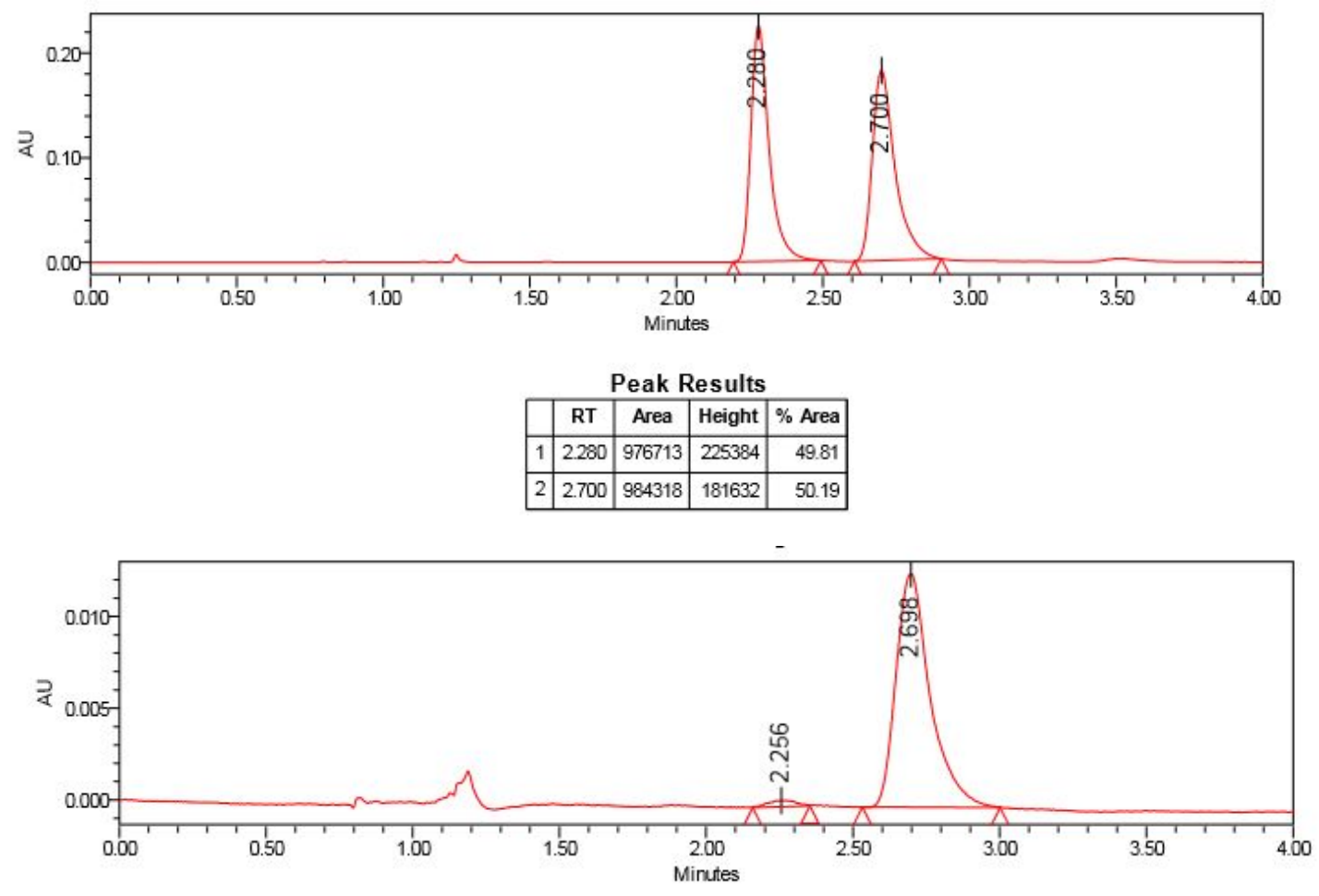

Peak Results

\begin{tabular}{|r|c|r|r|r|}
\hline & RT & Area & Height & $\%$ Area \\
\hline 1 & 2.256 & 2227 & 338 & 211 \\
\hline 2 & 2.698 & 103127 & 12732 & 97.89 \\
\hline
\end{tabular}


(2R,3S,3aR,9bS)-8-bromo-9b-methyl-2-(4-nitrophenyl)-3-phenyl-2,3,3a,9b-tetrah ydrochromeno[4,3-b]pyrrol-4(1H)-one $(4 \mathrm{~g})$ :

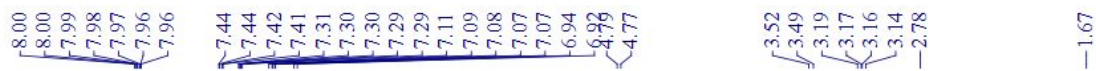
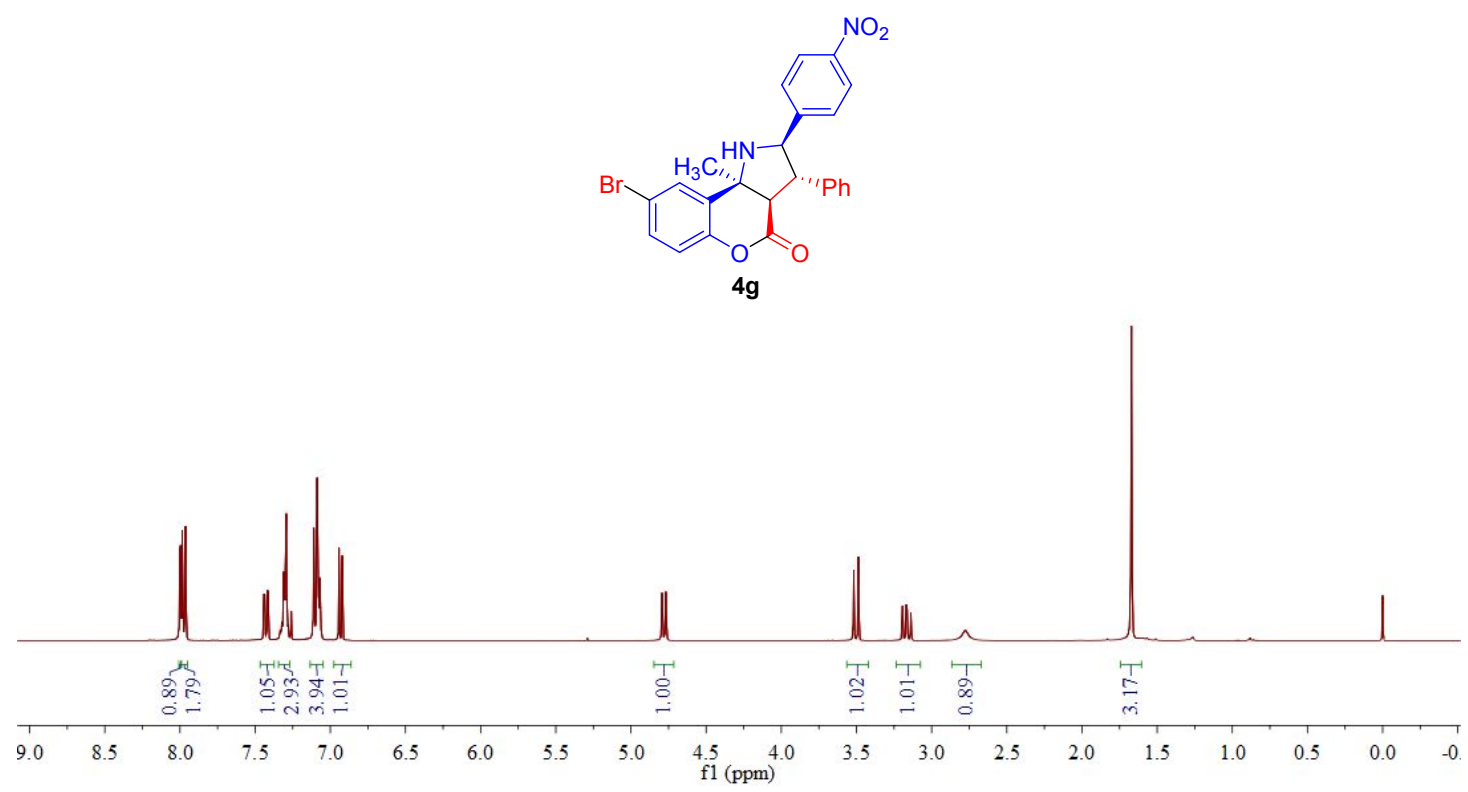

1
$\bar{b}$
$\overline{0}$

livin

i̊

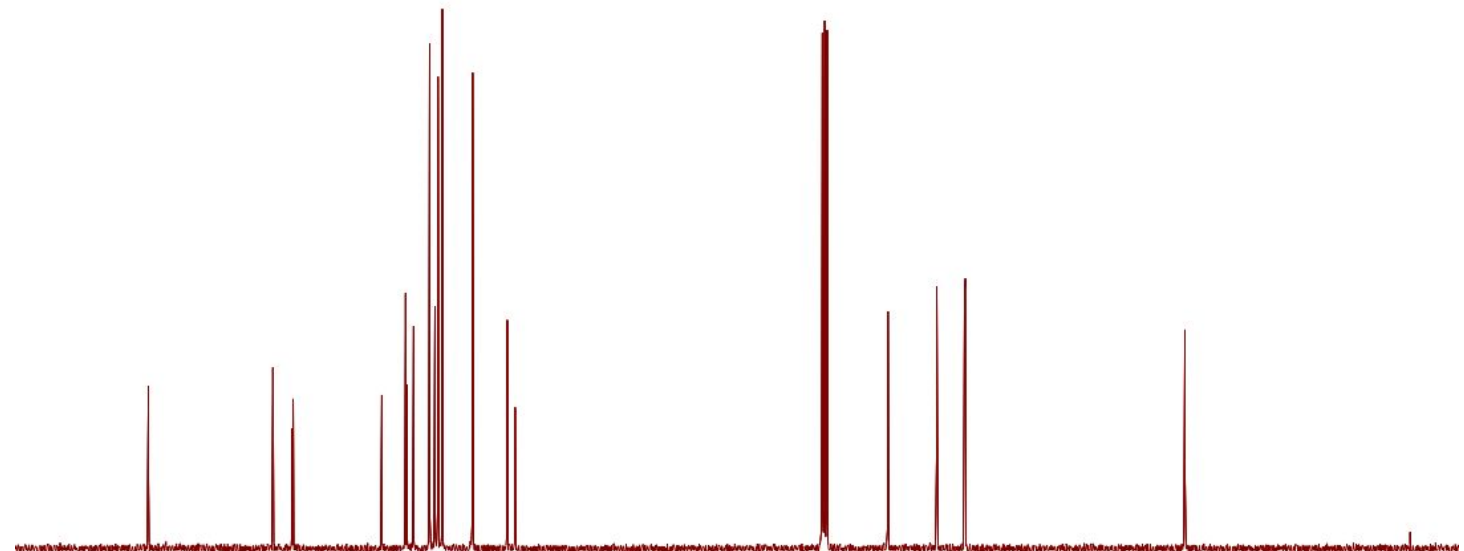

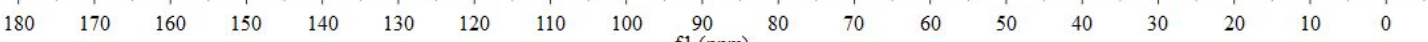




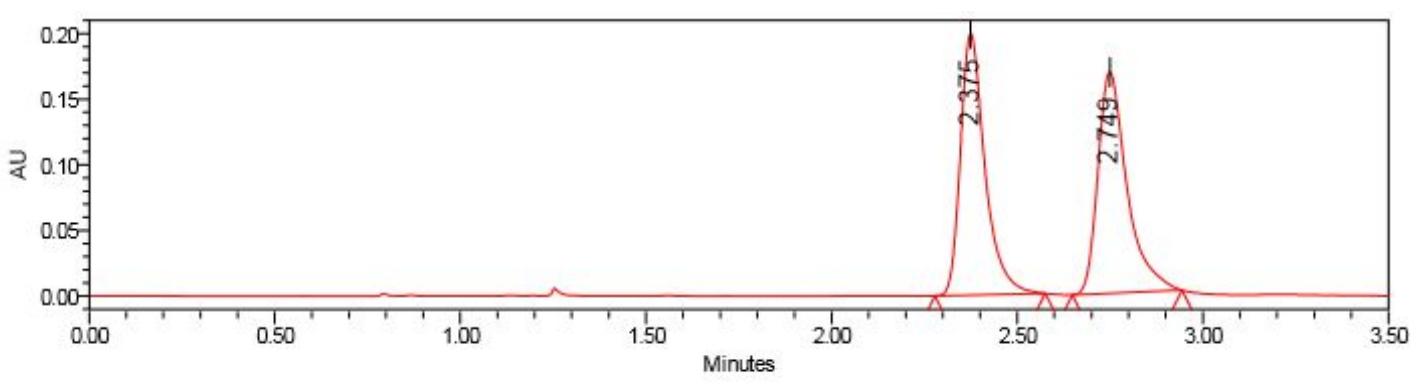

Peak Results

\begin{tabular}{|c|c|c|c|r|}
\hline & RT & Area & Height & $\%$ Area \\
\hline 1 & 2.375 & 914581 & 199231 & 49.87 \\
\hline 2 & 2.749 & 919259 & 168532 & 50.13 \\
\hline
\end{tabular}

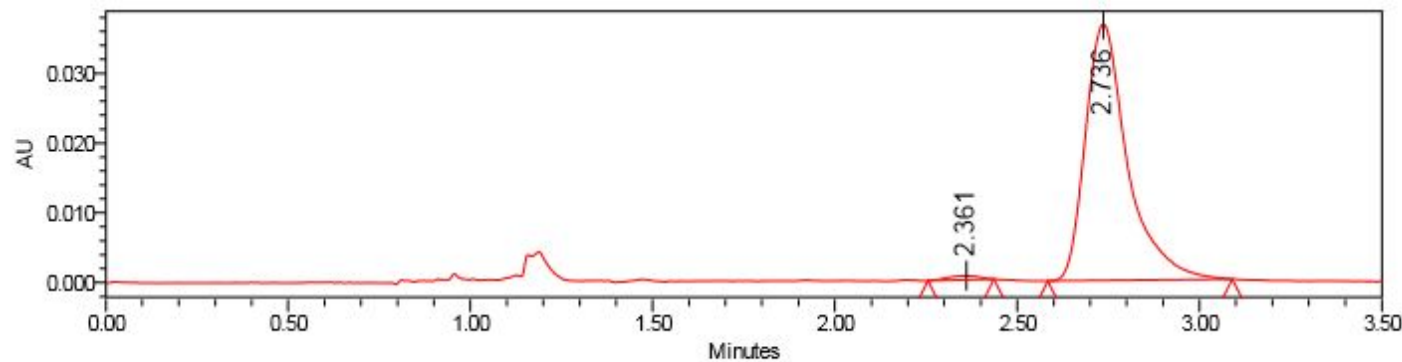

Peak Results
\begin{tabular}{|r|c|r|r|r|}
\hline & RT & Area & Height & $\%$ Area \\
\hline 1 & 2.361 & 3440 & 598 & 1.15 \\
\hline 2 & 2.736 & 295787 & 36748 & 98.85 \\
\hline
\end{tabular}


(2R,3S,3aR,9bS)-9b-methyl-8-nitro-2-(4-nitrophenyl)-3-phenyl-2,3,3a,9b-tetrahy drochromeno[4,3-b]pyrrol-4(1H)-one (4h):

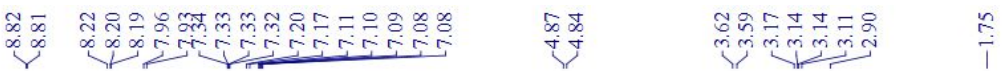
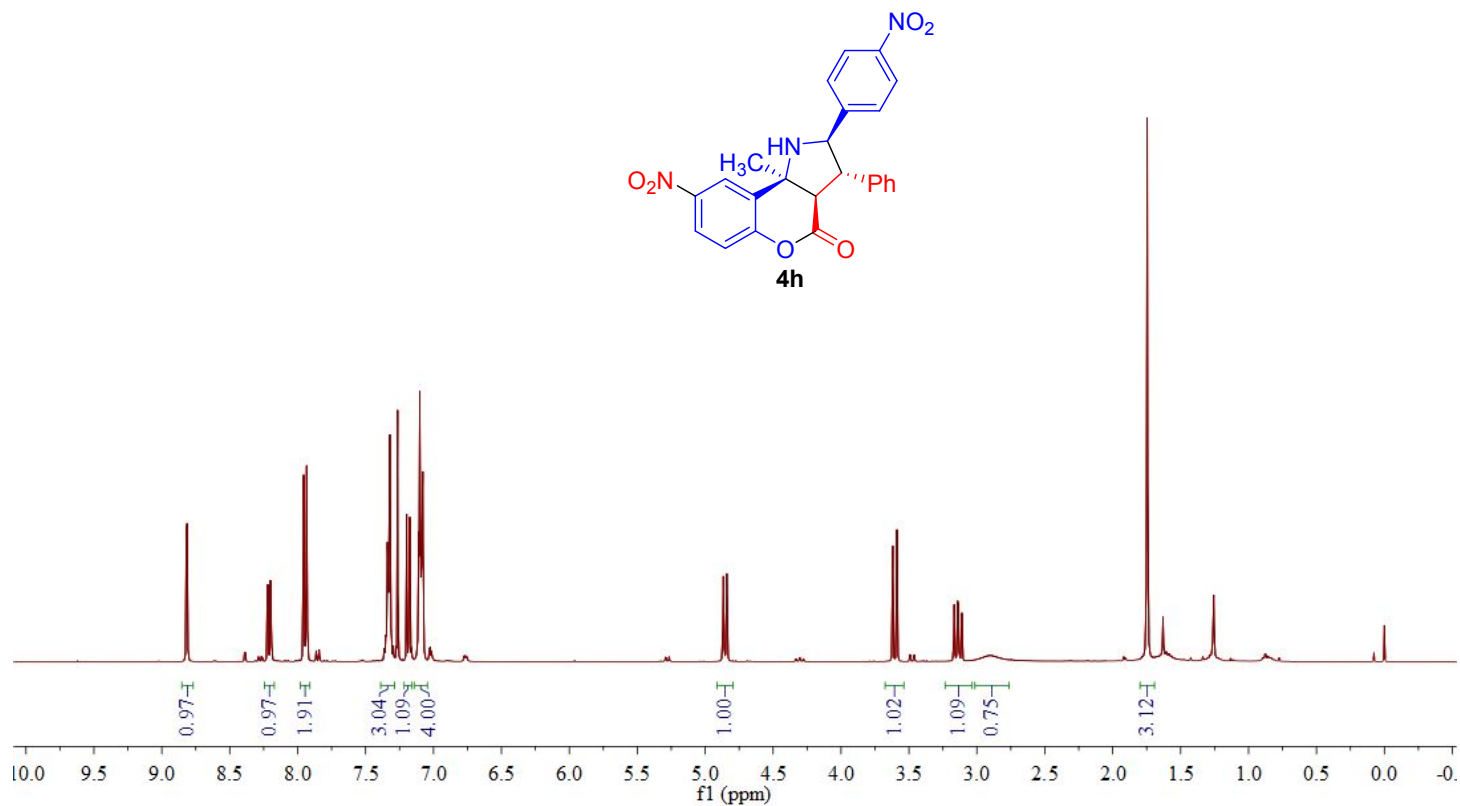

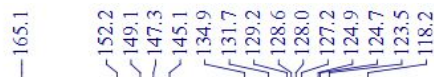

i

$\stackrel{2}{i}$

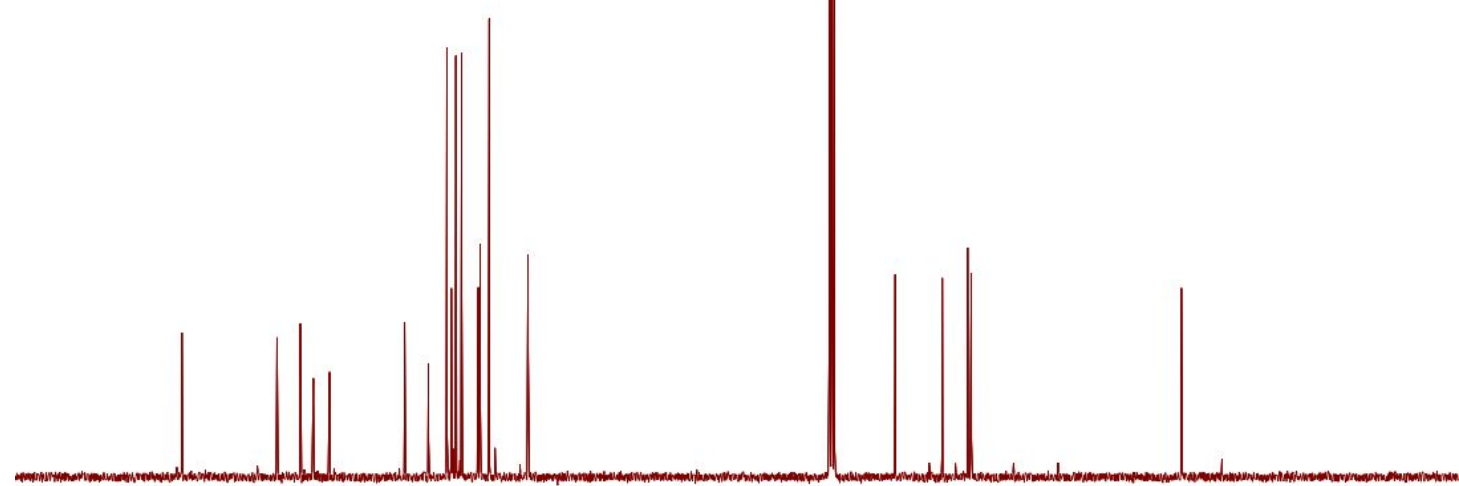

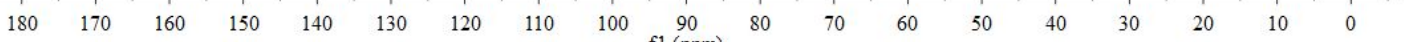




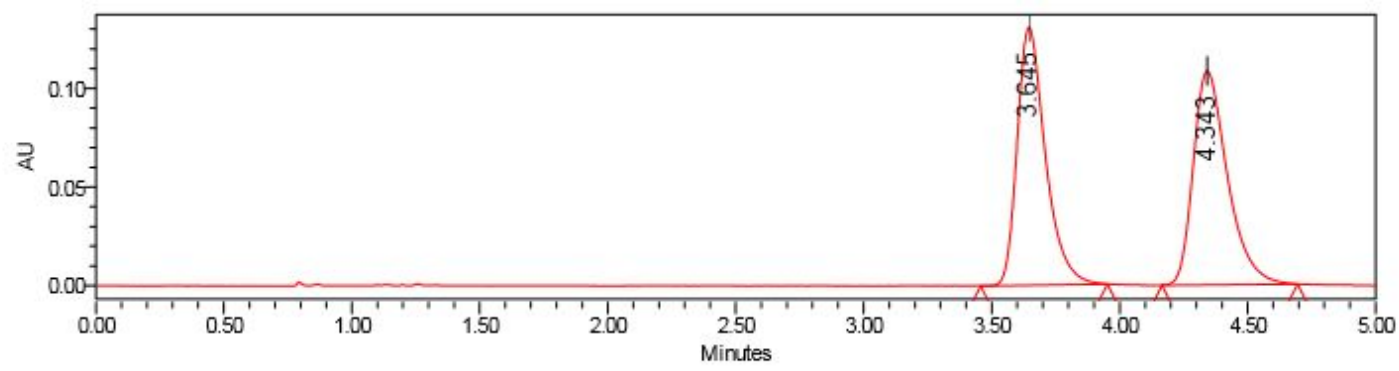

Peak Results

\begin{tabular}{|c|c|c|c|r|}
\hline & RT & Area & Height & $\%$ Area \\
\hline 1 & 3.645 & 1001037 & 130691 & 49.86 \\
\hline 2 & 4.343 & 1006640 & 108432 & 50.14 \\
\hline
\end{tabular}

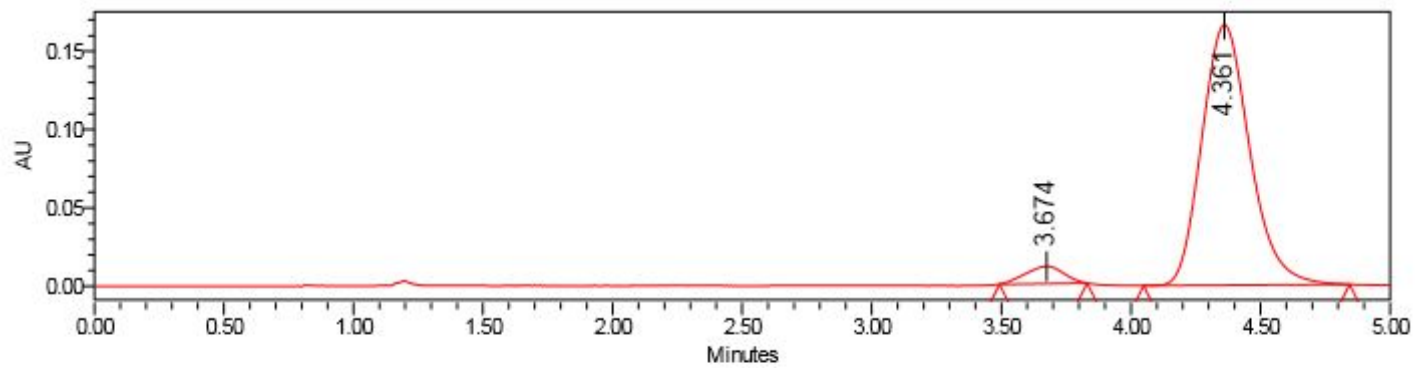

Peak Results

\begin{tabular}{|r|c|c|r|r|}
\hline & RT & Area & Height & $\%$ Area \\
\hline 1 & 3.674 & 112329 & 11046 & 5.00 \\
\hline 2 & 4.361 & 2133771 & 166200 & 95.00 \\
\hline
\end{tabular}


(2R,3S,3aR,9bS)-8,9b-dimethyl-2-(4-nitrophenyl)-3-phenyl-2,3,3a,9b-tetrahydroc hromeno[4,3-b]pyrrol-4(1H)-one (4i):

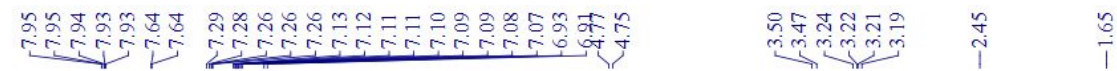
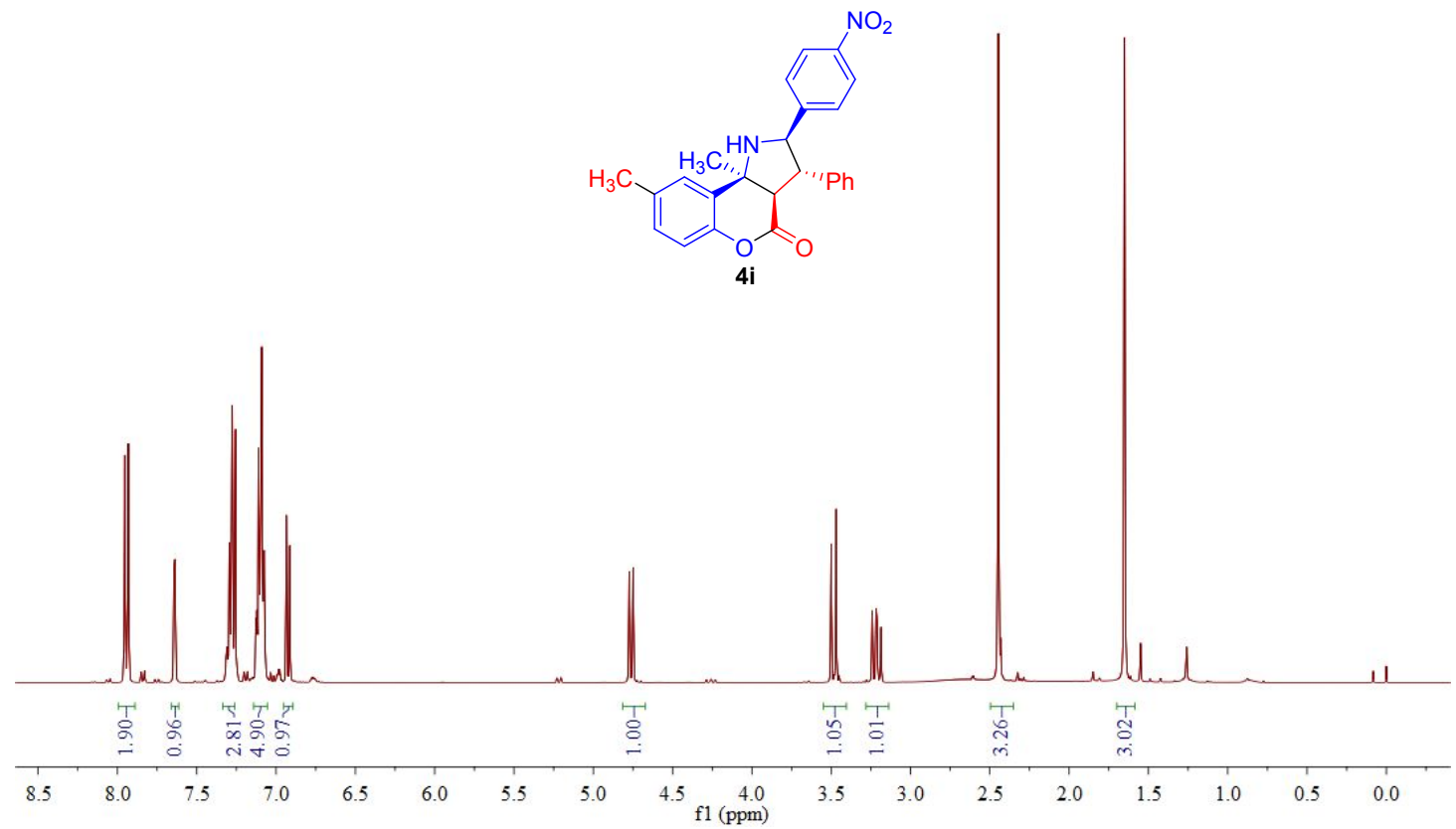

阝

in

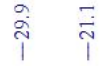

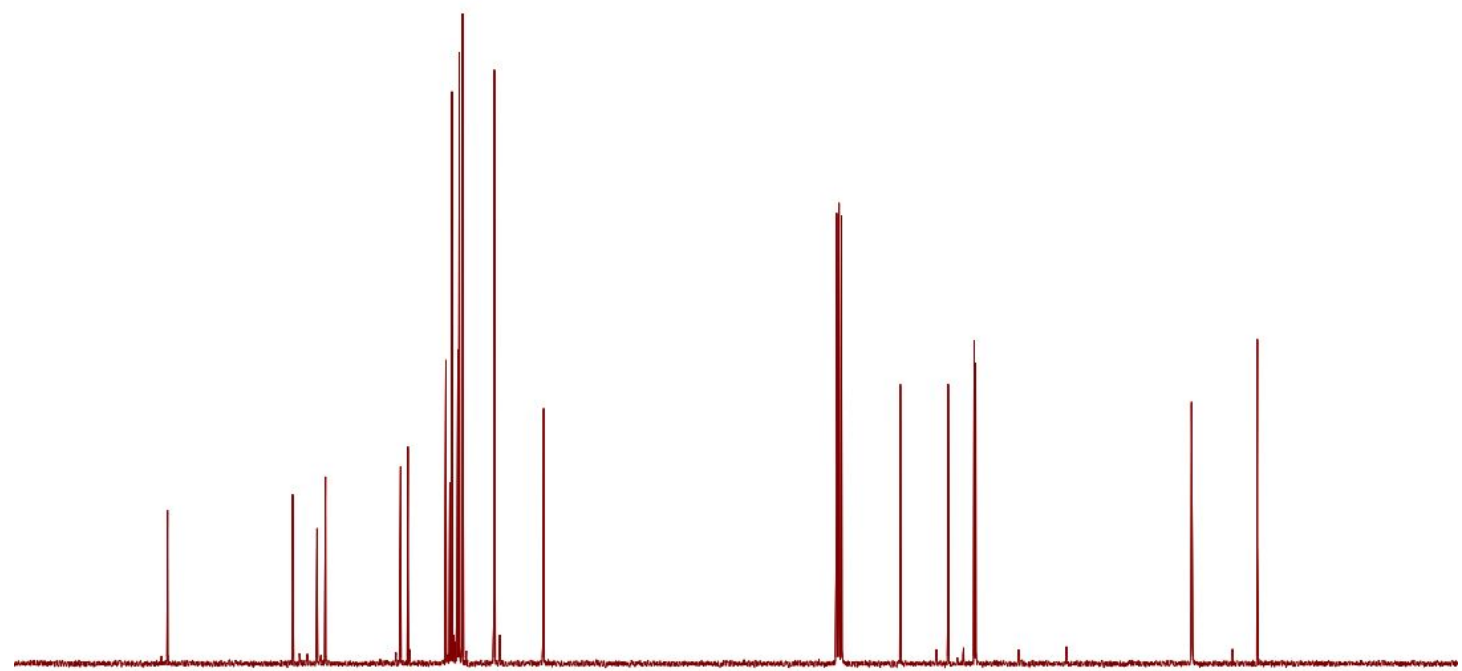

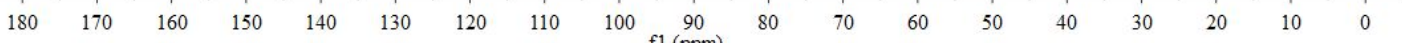




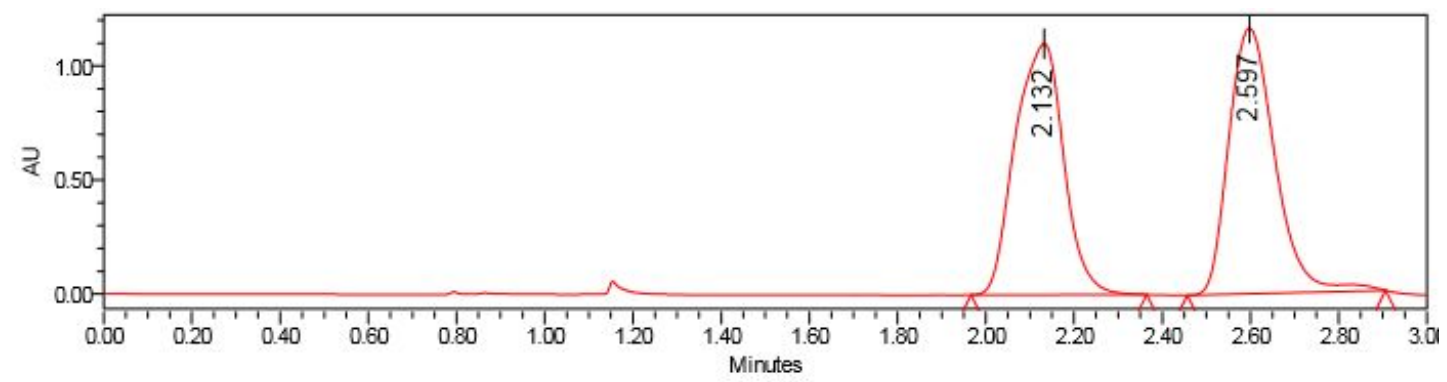

Peak Results

\begin{tabular}{|c|c|c|c|r|}
\hline & RT & Area & Height & $\%$ Area \\
\hline 1 & 2132 & 8429765 & 1101046 & 49.89 \\
\hline 2 & 2597 & 8467458 & 1164739 & 50.11 \\
\hline
\end{tabular}

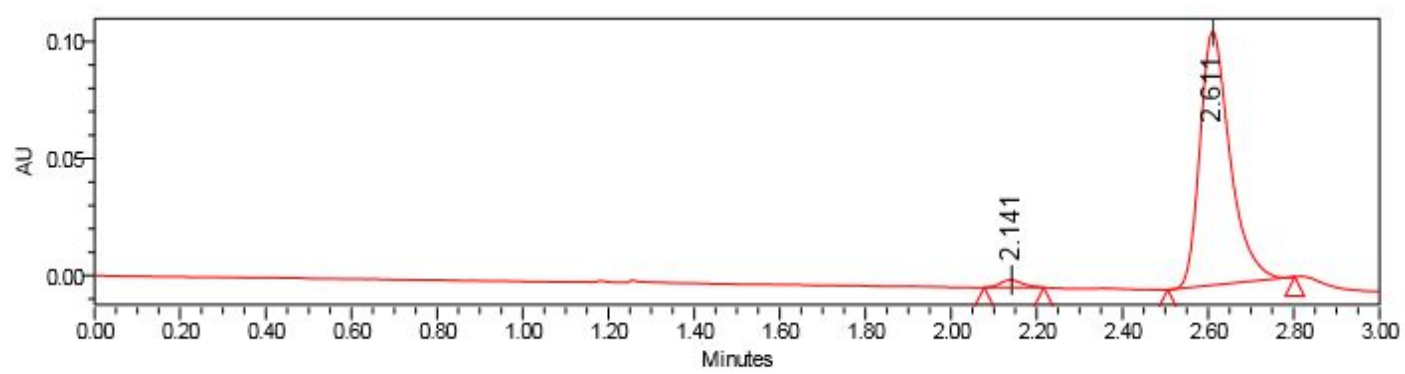

Peak Results

\begin{tabular}{|r|c|r|r|r|}
\hline & RT & Area & Height & $\%$ Area \\
\hline 1 & 2141 & 11535 & 3192 & 2.13 \\
\hline 2 & 2611 & 530089 & 108450 & 97.87 \\
\hline
\end{tabular}


(2R,3S,3aR,9bS)-9-methoxy-9b-methyl-2-(4-nitrophenyl)-3-phenyl-2,3,3a,9b-tetr ahydrochromeno[4,3-b]pyrrol-4(1H)-one $(4 \mathrm{j})$ :

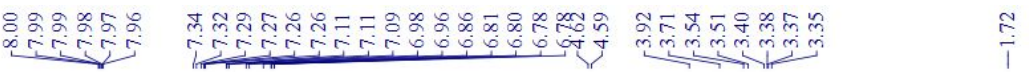

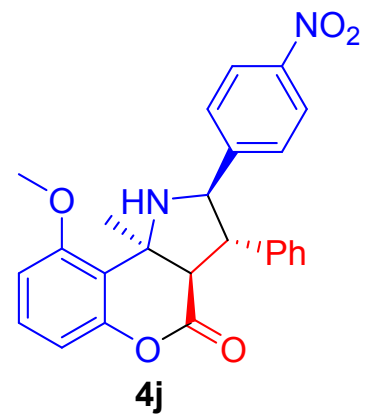

$4 \mathbf{j}$

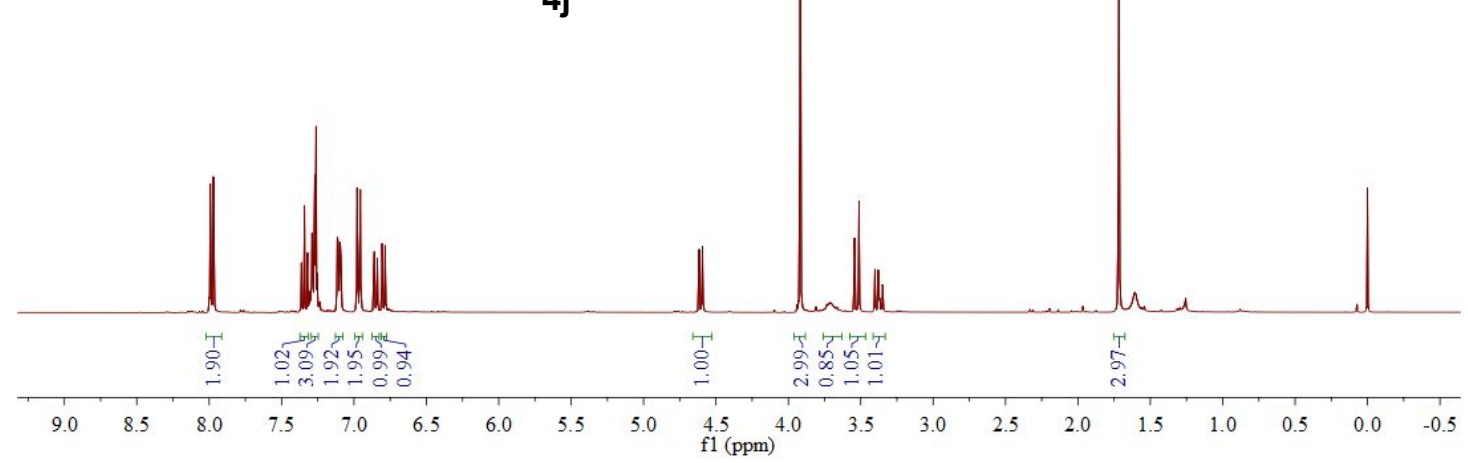

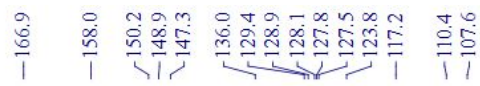

gic

$\stackrel{\sim}{\sim}$

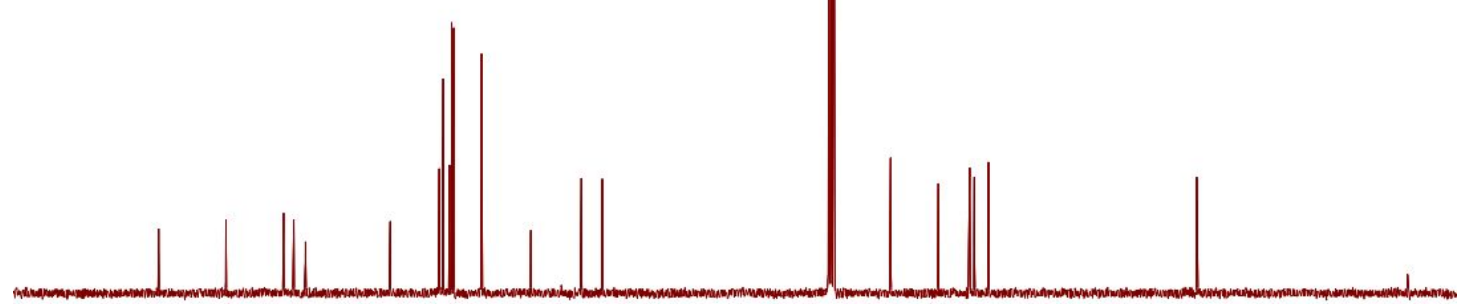

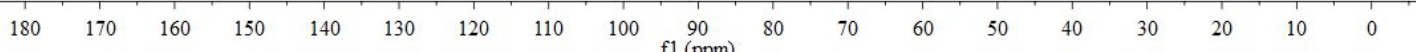




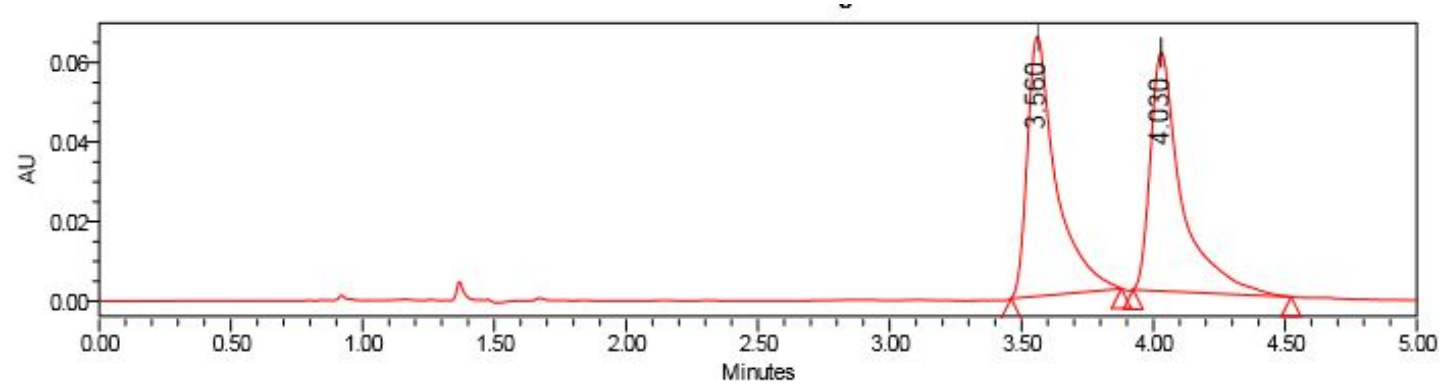

Peak Results

\begin{tabular}{|c|c|c|r|r|}
\hline & RT & Area & Height & $\%$ Area \\
\hline 1 & 3.560 & 494881 & 65317 & 50.00 \\
\hline 2 & 4.030 & 494871 & 60014 & 50.00 \\
\hline
\end{tabular}

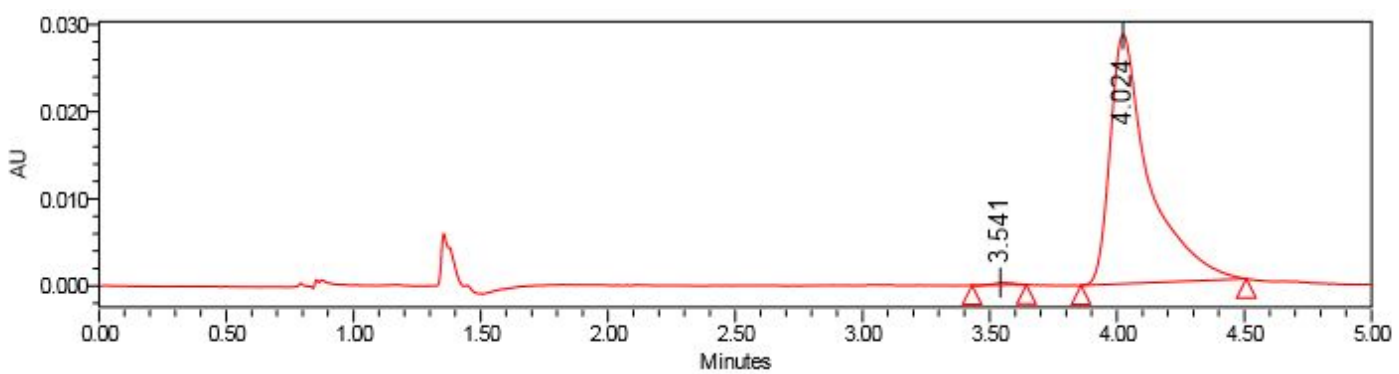

Peak Results

\begin{tabular}{|r|c|r|r|r|}
\hline & RT & Area & Height & $\%$ Area \\
\hline 1 & 3.541 & 1488 & 270 & 0.49 \\
\hline 2 & 4.024 & 304333 & 28608 & 99.51 \\
\hline
\end{tabular}


(2R,3S,3aR,9bS)-9b-ethyl-2-(4-nitrophenyl)-3-phenyl-2,3,3a,9b-tetrahydrochrom eno[4,3-b]pyrrol-4(1H)-one (4k):

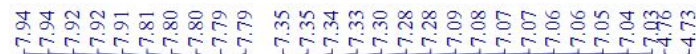

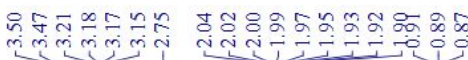

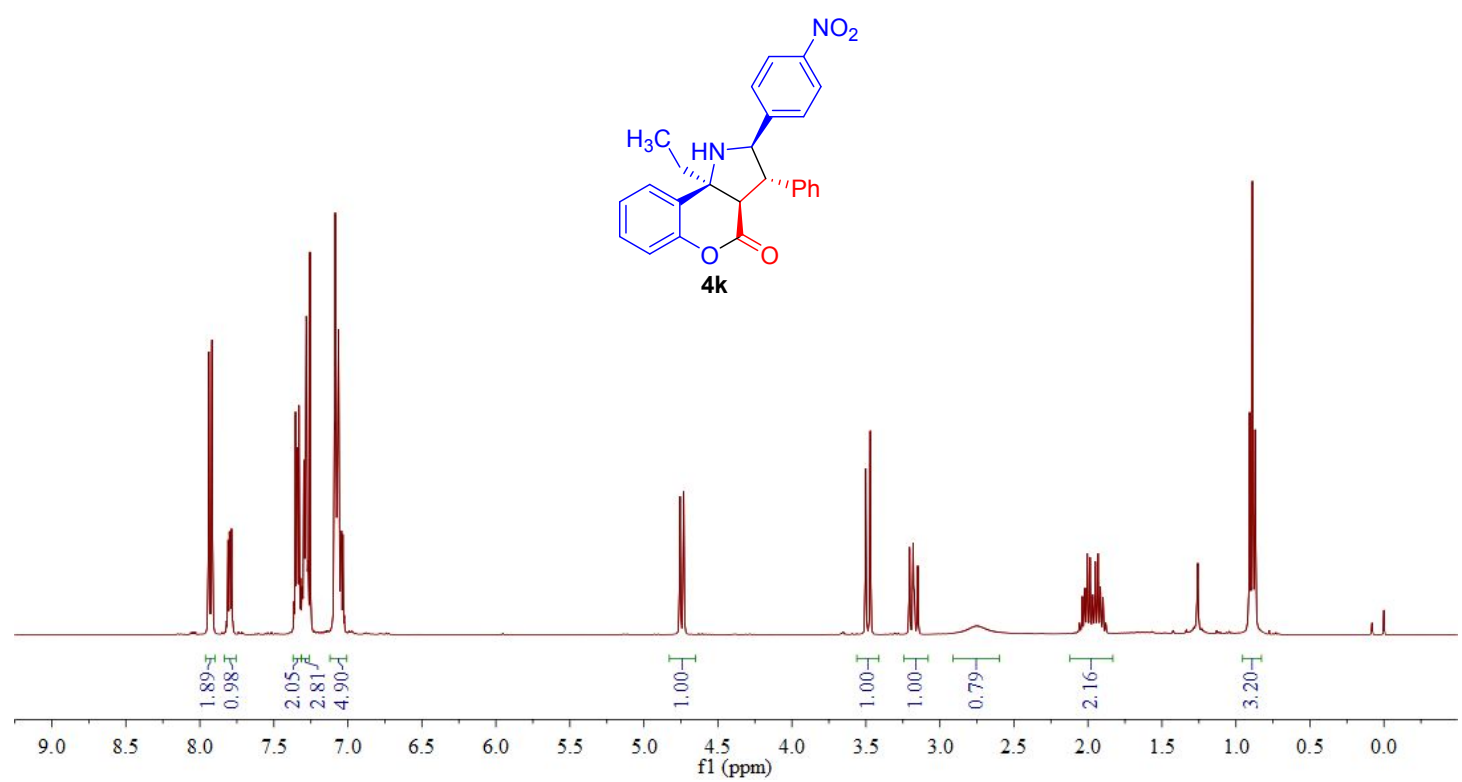

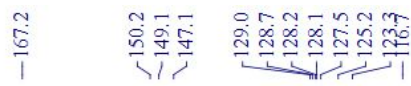

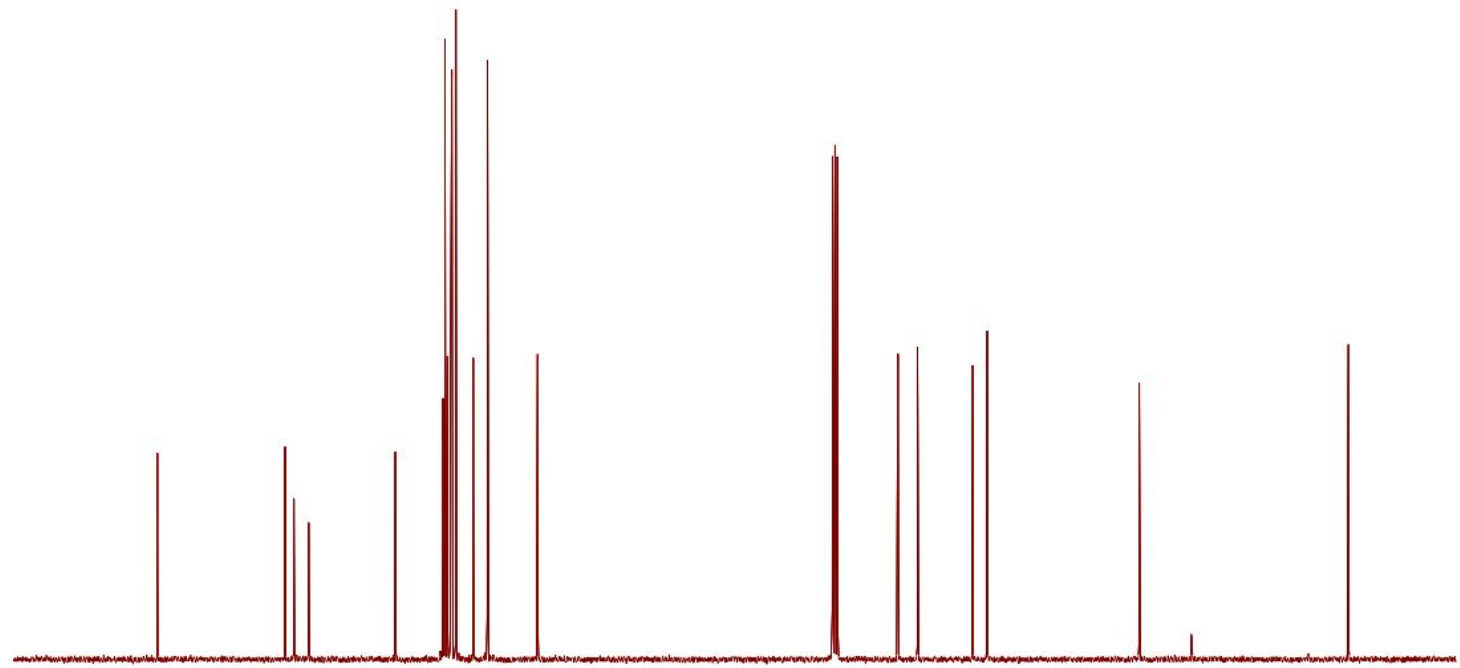

$\begin{array}{lllllllllllllllllll}180 & 170 & 160 & 150 & 140 & 130 & 120 & 110 & 100 & 90 & 80 & 70 & 60 & 50 & 40 & 30 & 20 & 10 & 0\end{array}$ 


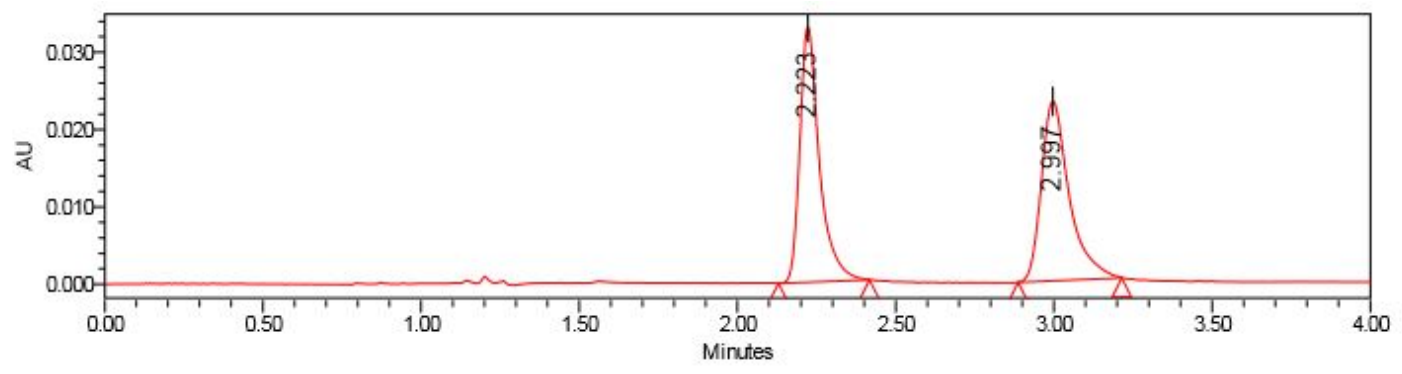

Peak Results

\begin{tabular}{|c|c|c|r|r|}
\hline & RT & Area & Height & $\%$ Area \\
\hline 1 & 2.223 & 141774 & 32961 & 49.92 \\
\hline 2 & 2.997 & 142218 & 23227 & 50.06 \\
\hline
\end{tabular}

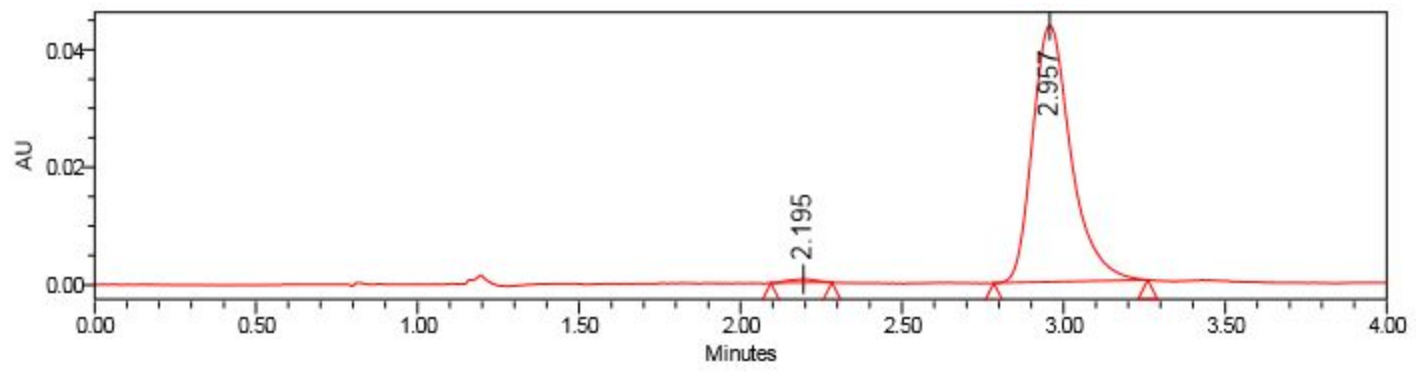

Peak Results

\begin{tabular}{|r|c|r|r|r|}
\hline & RT & Area & Height & $\%$ Area \\
\hline 1 & 2.195 & 3795 & 630 & 1.04 \\
\hline 2 & 2.957 & 361655 & 43645 & 98.96 \\
\hline
\end{tabular}


(2R,3S,3aR,9bR)-2-(4-nitrophenyl)-3,9b-diphenyl-2,3,3a,9b-tetrahydrochromeno[ 4,3-b]pyrrol-4(1H)-one (4I):

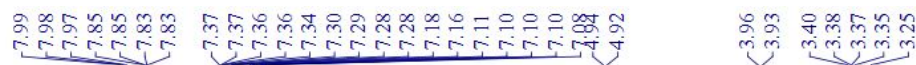

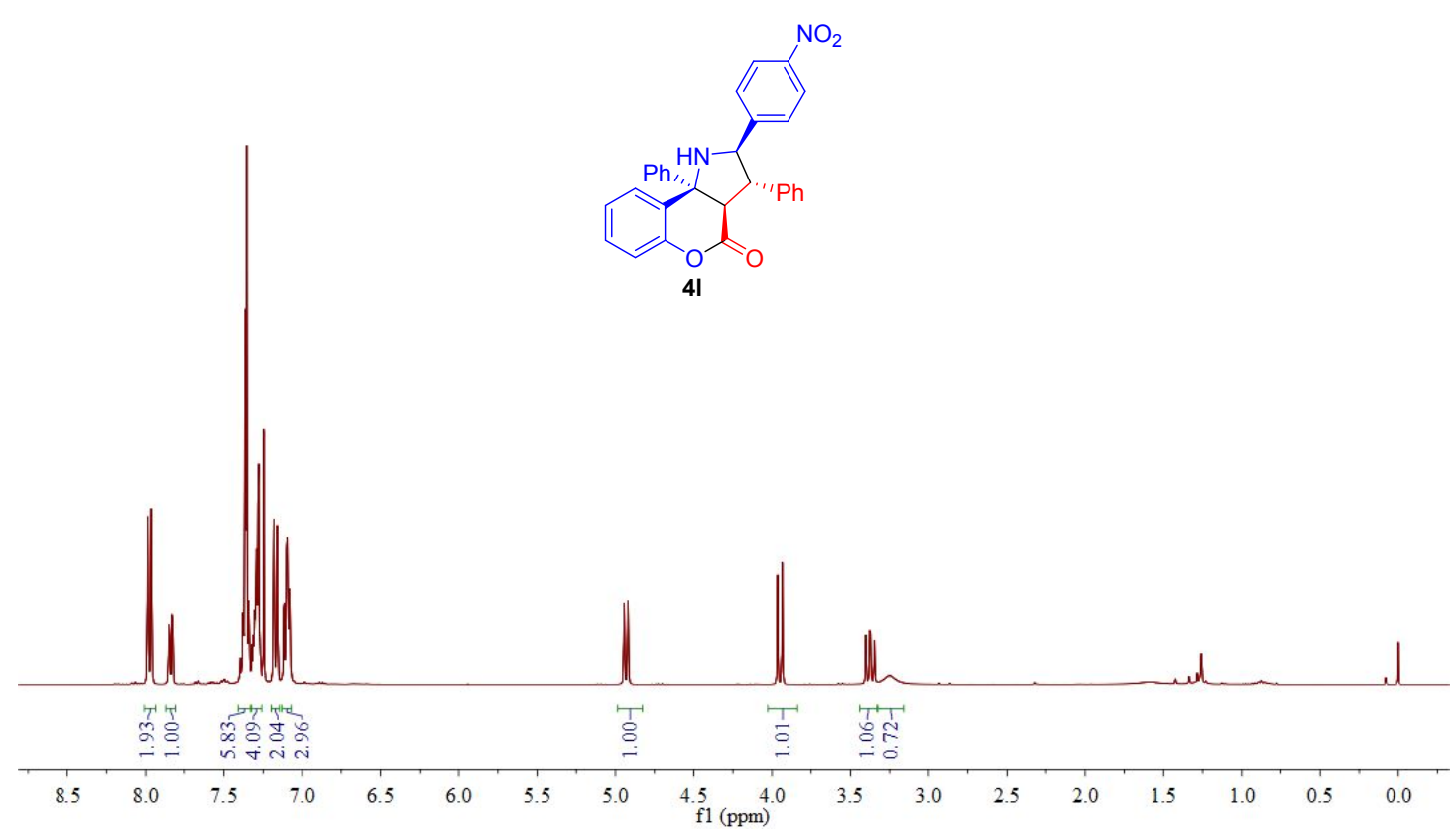

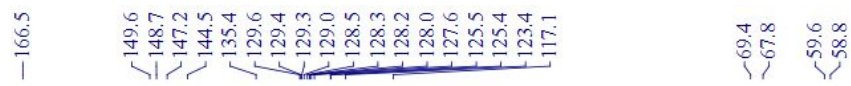

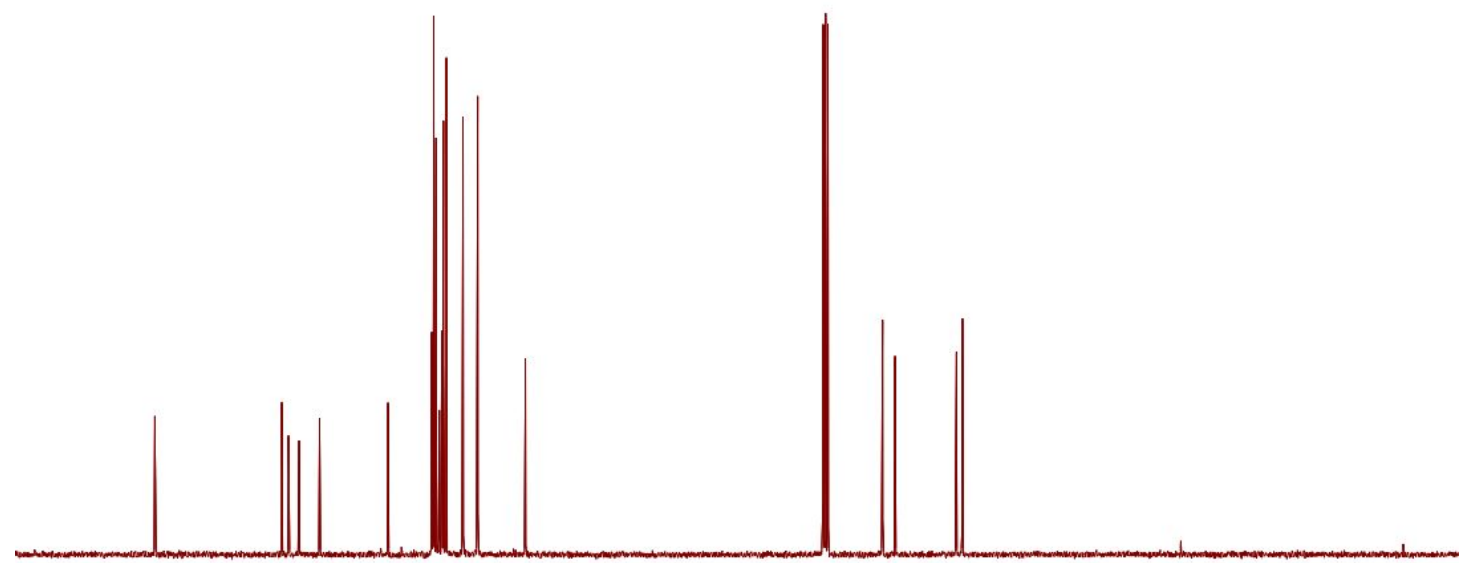

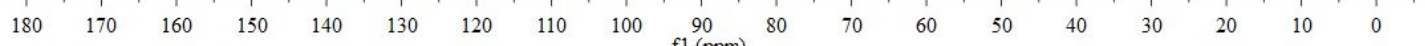




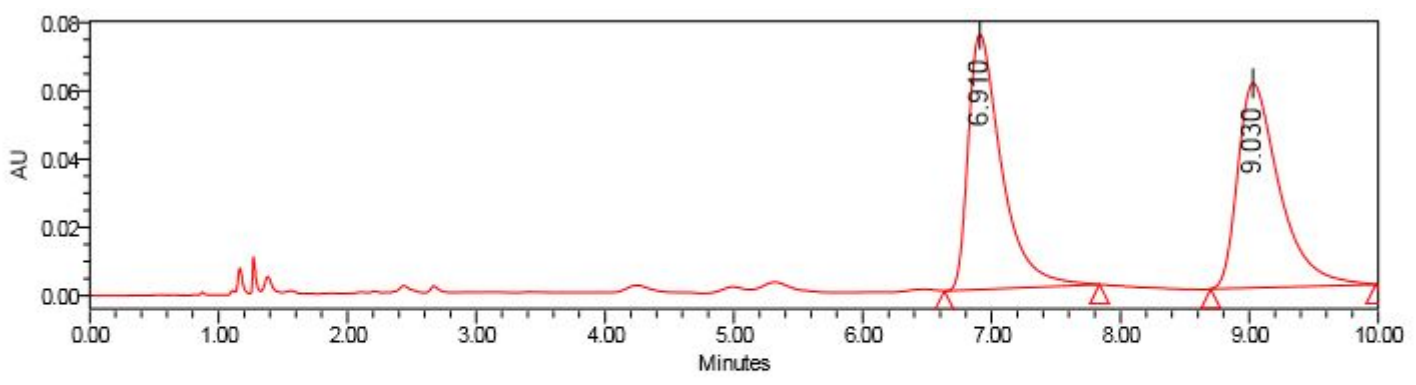

Peak Results

\begin{tabular}{|c|c|c|r|r|}
\hline & RT & Area & Height & $\%$ Area \\
\hline 1 & 6.910 & 1342460 & 74686 & 49.96 \\
\hline 2 & 9.030 & 1344733 & 59906 & 50.04 \\
\hline
\end{tabular}

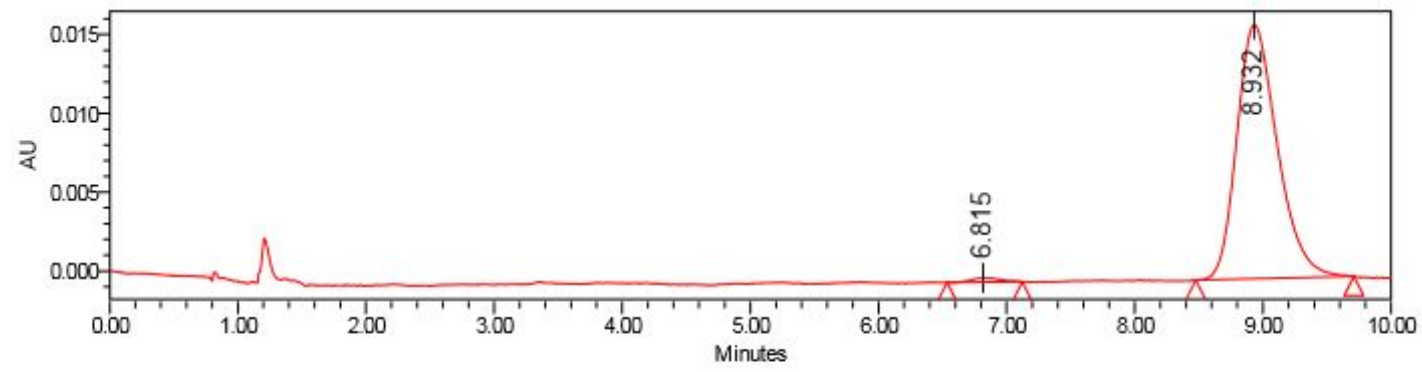

Peak Results

\begin{tabular}{|r|c|r|r|r|}
\hline & RT & Area & Height & $\%$ Area \\
\hline 1 & 6.815 & 4117 & 273 & 1.15 \\
\hline 2 & 8.932 & 354406 & 16146 & 98.85 \\
\hline
\end{tabular}


methyl(2S,3R,4S,5R)-2-(2-hydroxyphenyl)-2-methyl-5-(4-nitrophenyl)-4-phenylp yrrolidine-3-carboxylate (5):

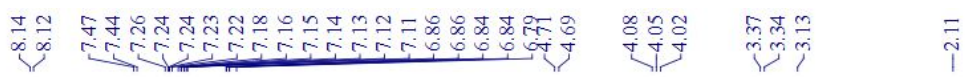
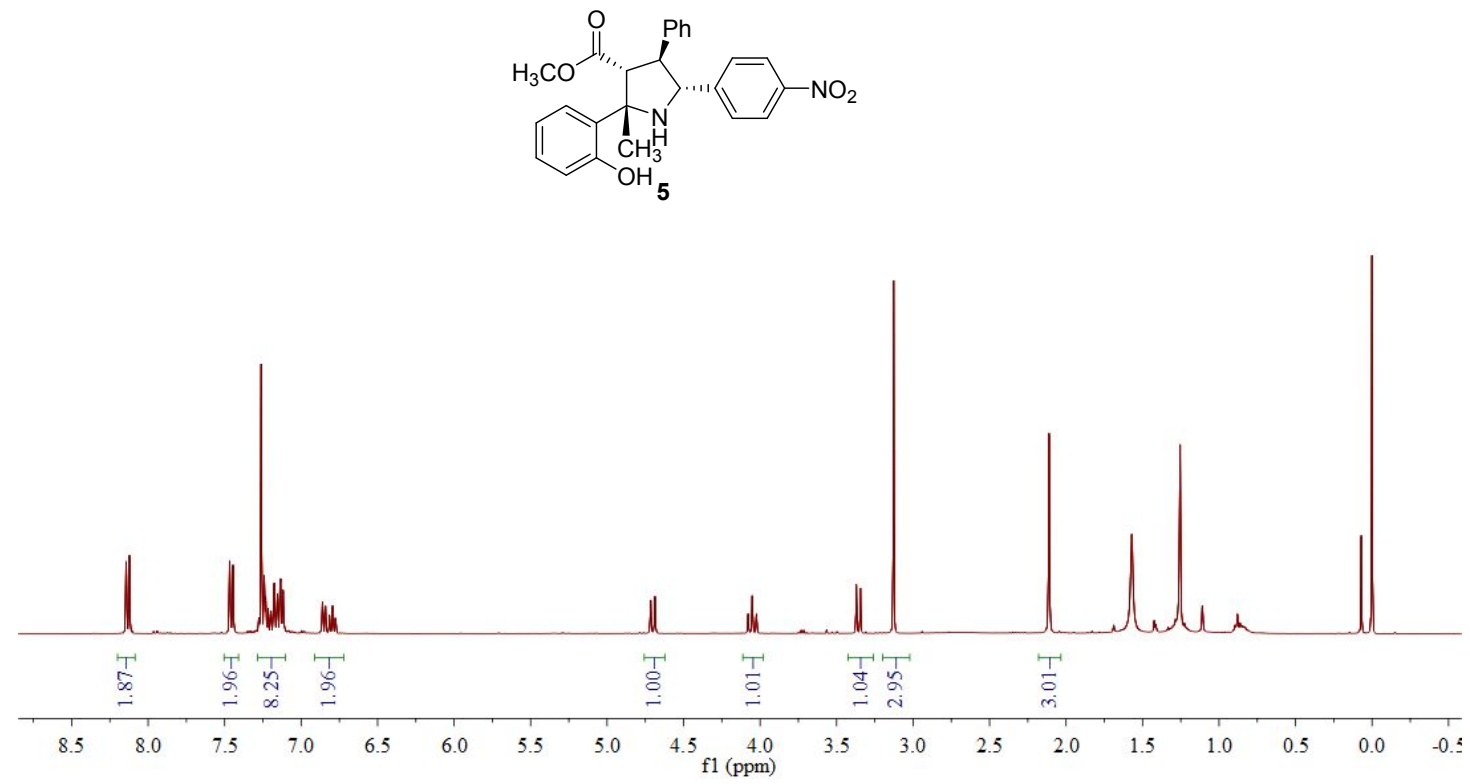

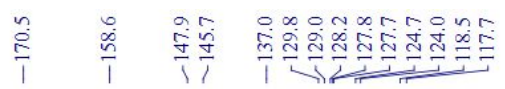

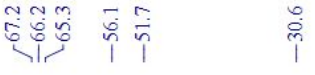
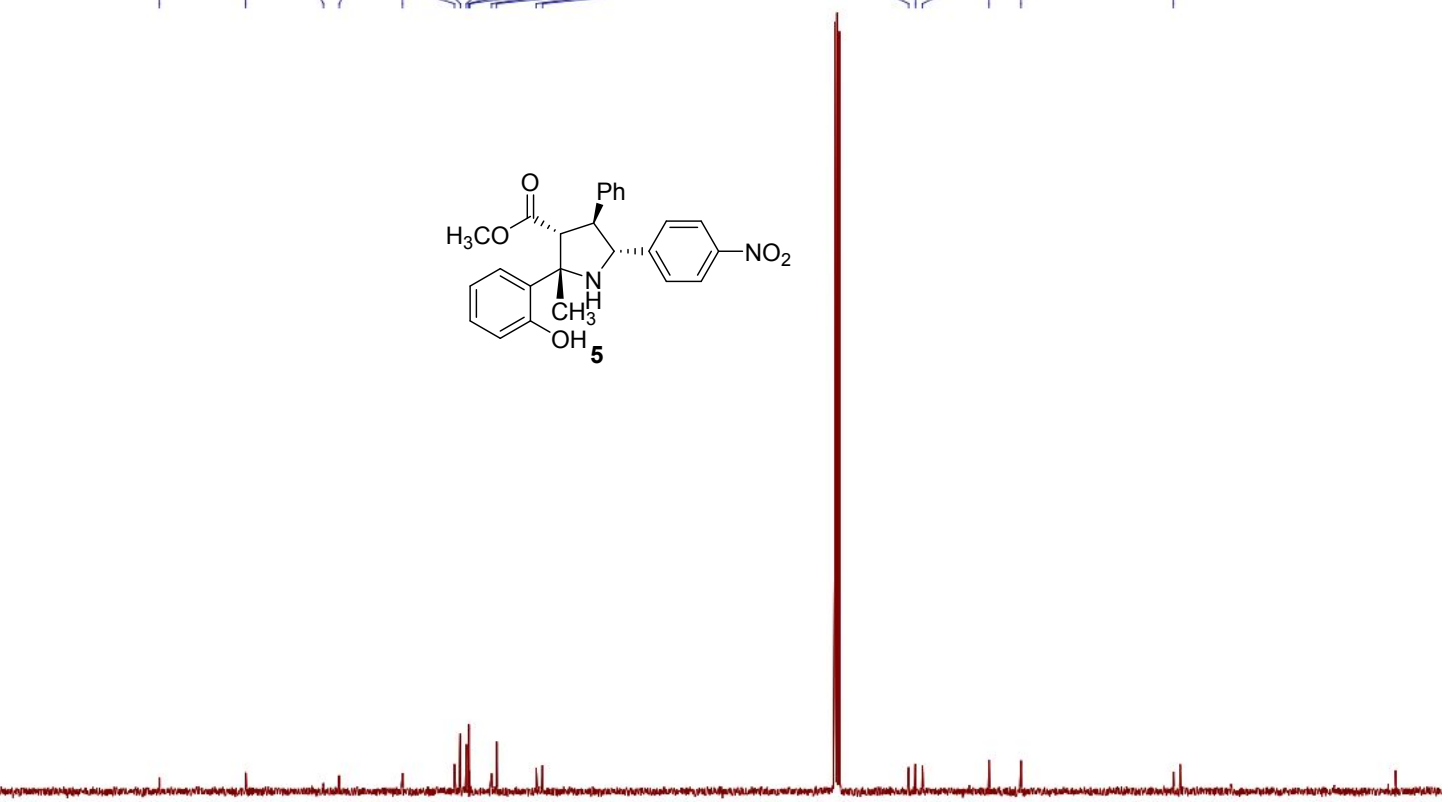

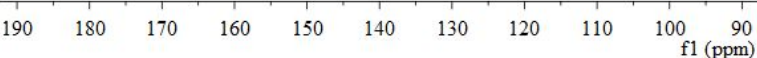



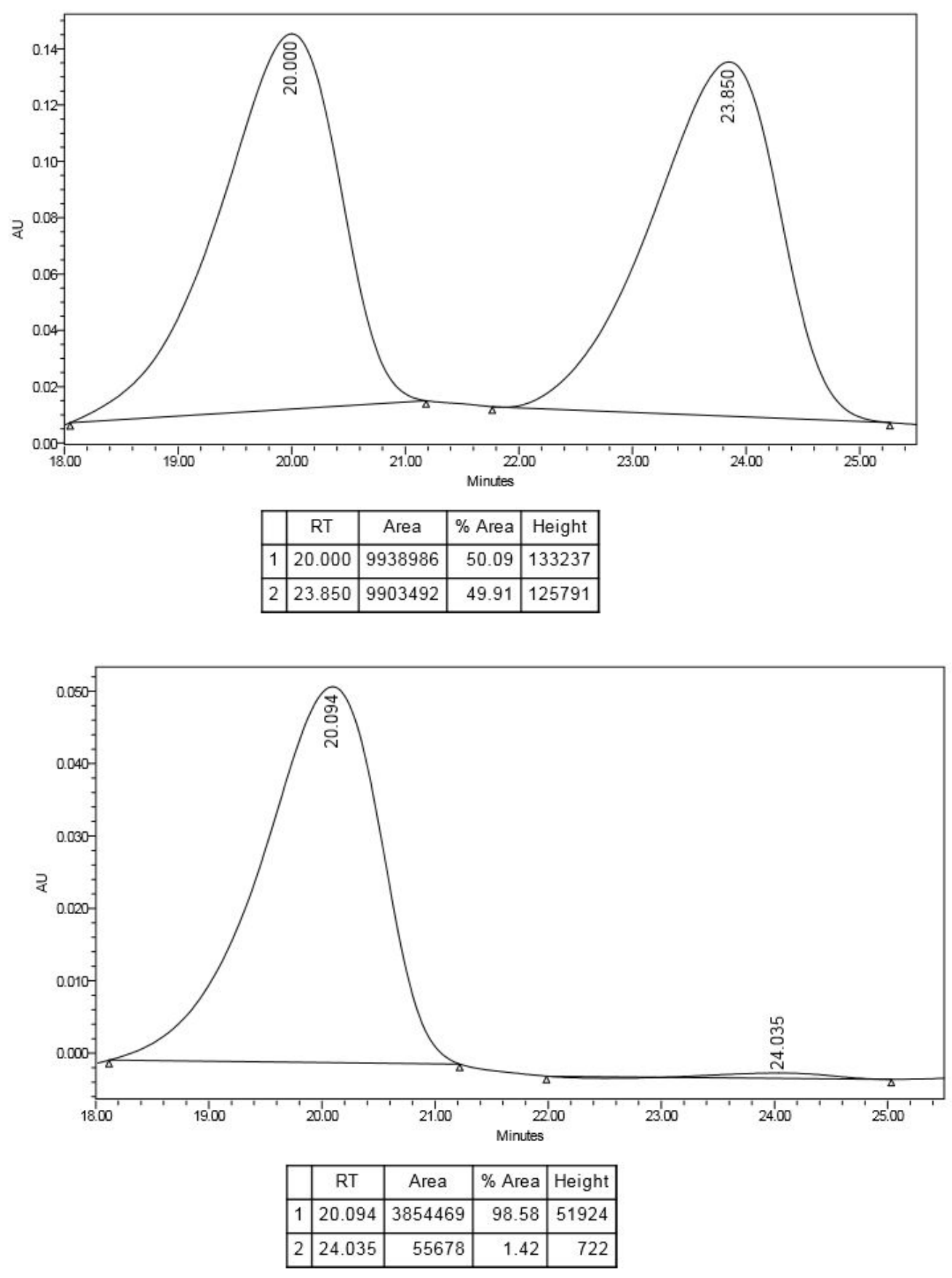
methyl(2S,3R,4S,5R)-5-(4-aminophenyl)-2-(2-hydroxyphenyl)-2-methyl-4-phenyl pyrrolidine-3-carboxylate (6)

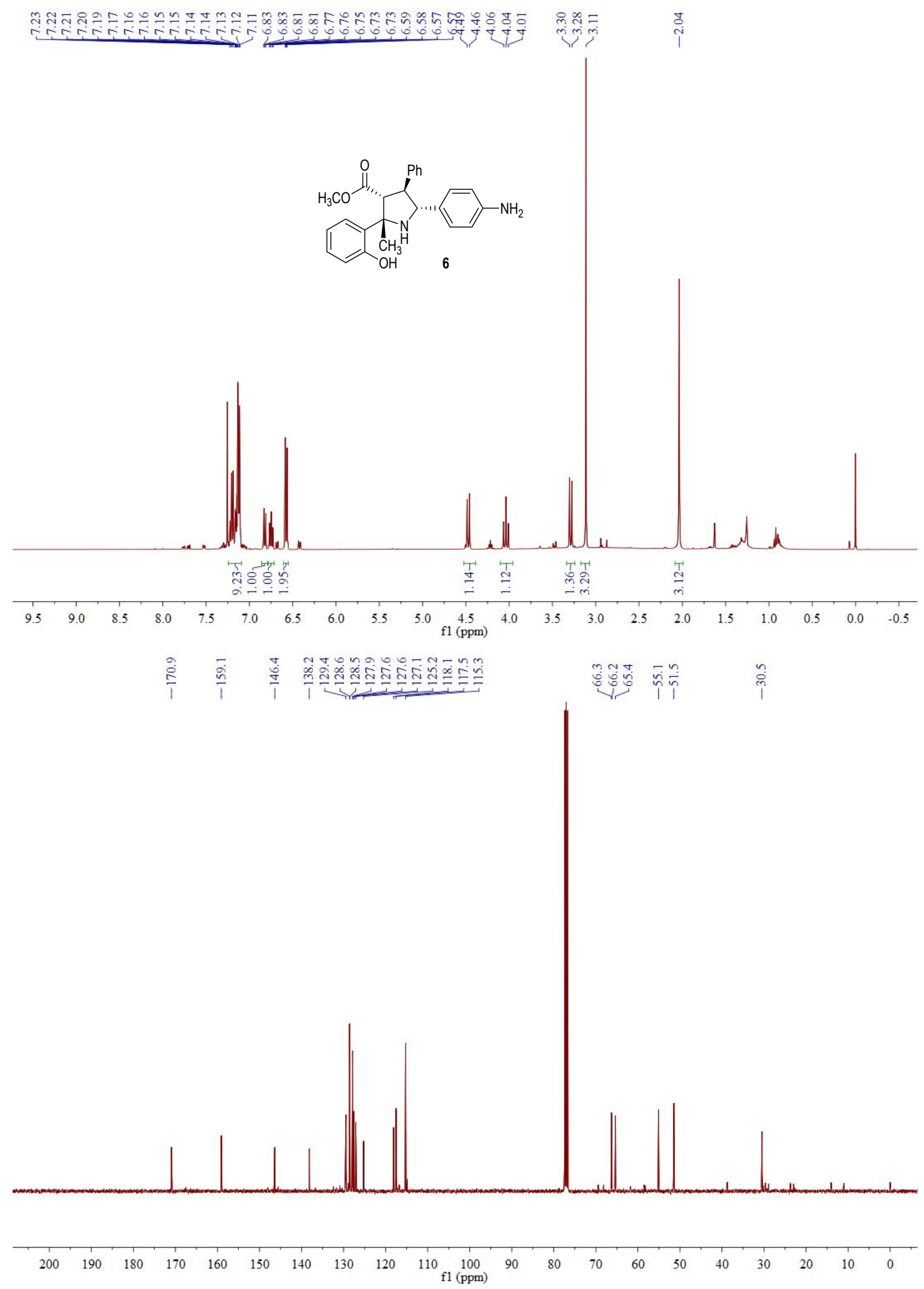


methyl(2S,3R,4S,5R)-5-(4-(3-(3,5-bis(trifluoromethyl)phenyl)thioureido)phenyl)2-(2-hydroxyphenyl)-2-methyl-4-phenylpyrrolidine-3-carboxylate(7)

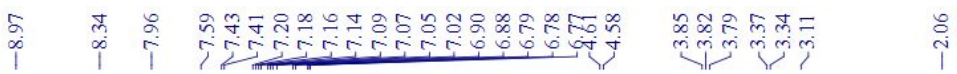

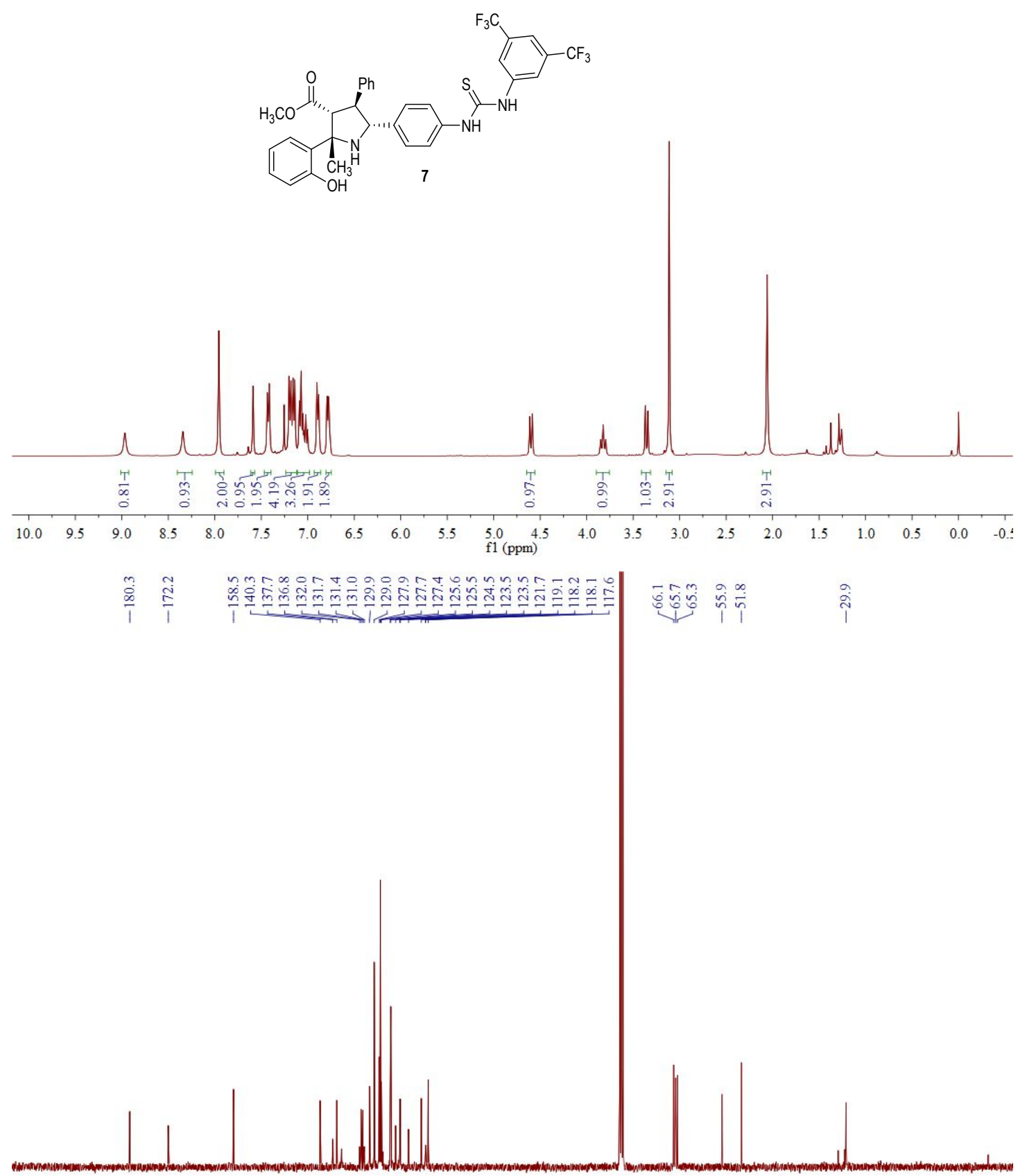

$\begin{array}{lllllllllllllllllllll}200 & 190 & 180 & 170 & 160 & 150 & 140 & 130 & 120 & 110 & 100 & 90 & 80 & 70 & 60 & 50 & 40 & 30 & 20 & 10 & 0\end{array}$ 

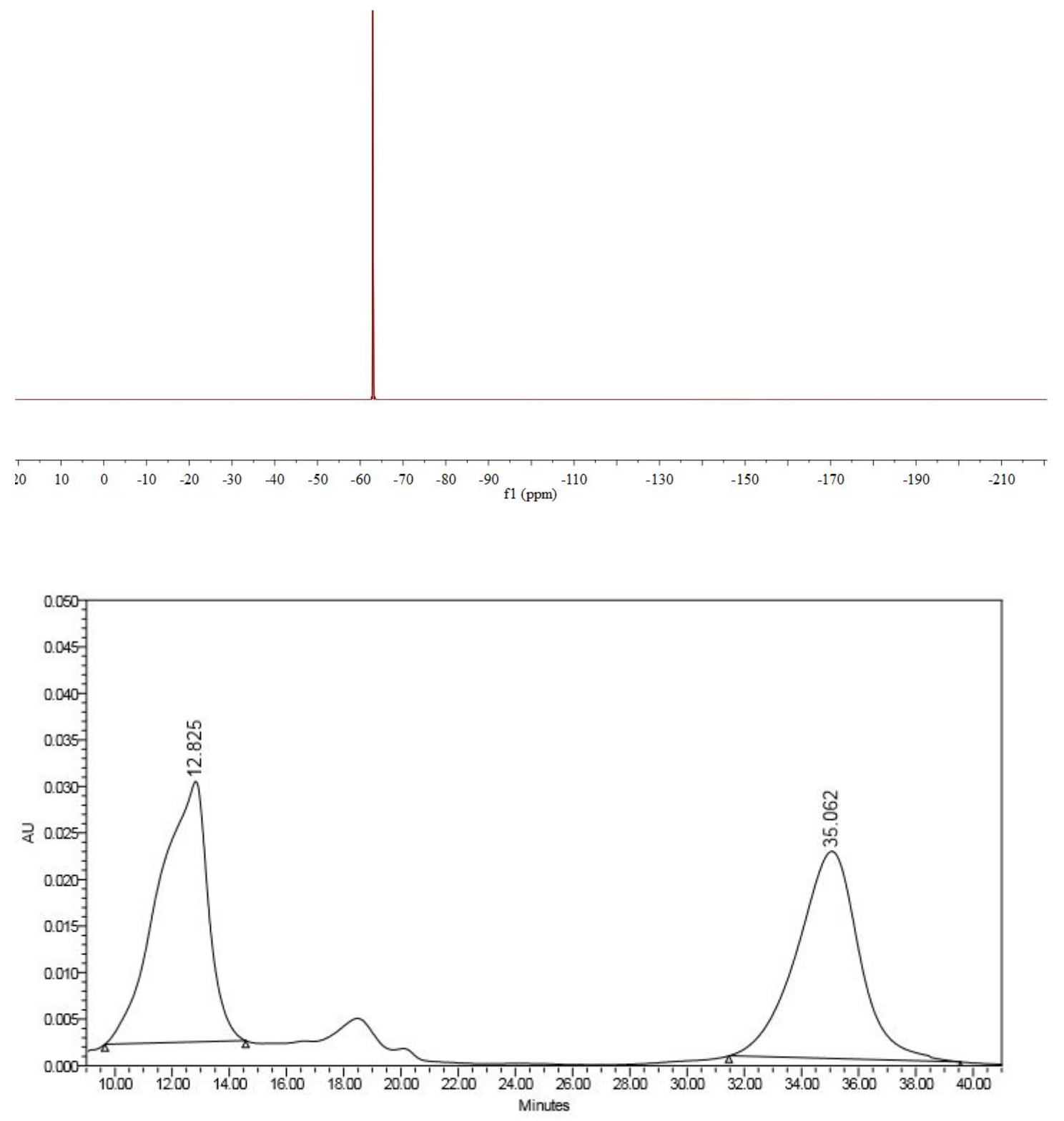

\begin{tabular}{|c|c|c|r|c|}
\hline & RT & Area & \% Area & Height \\
\hline 1 & 12.825 & 3386477 & 50.13 & 27974 \\
\hline 2 & 35.062 & 3369337 & 49.87 & 22253 \\
\hline
\end{tabular}




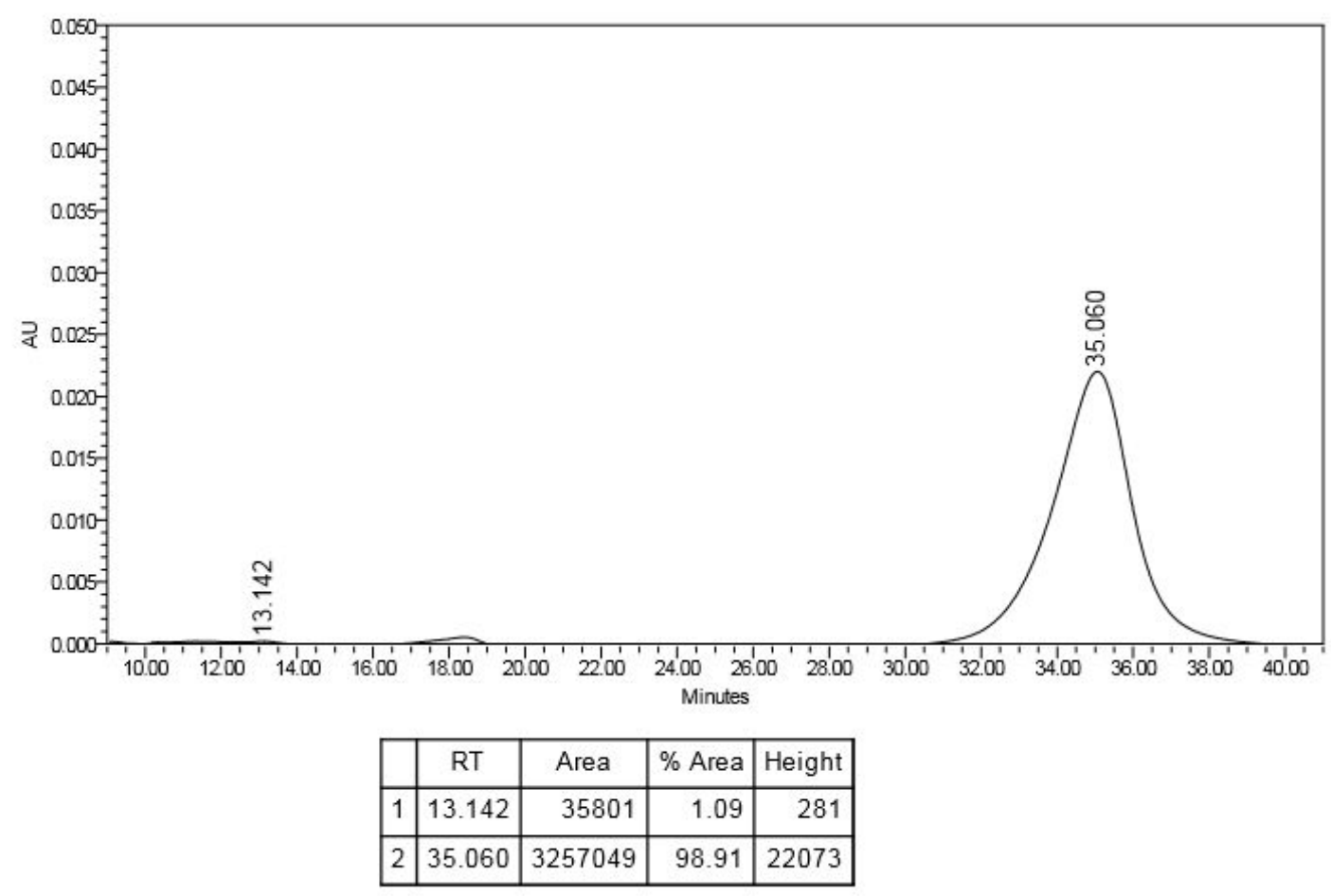




\section{${ }^{1} \mathrm{H}$ NMR of H-D exchanging studies}

$\frac{8}{1}$
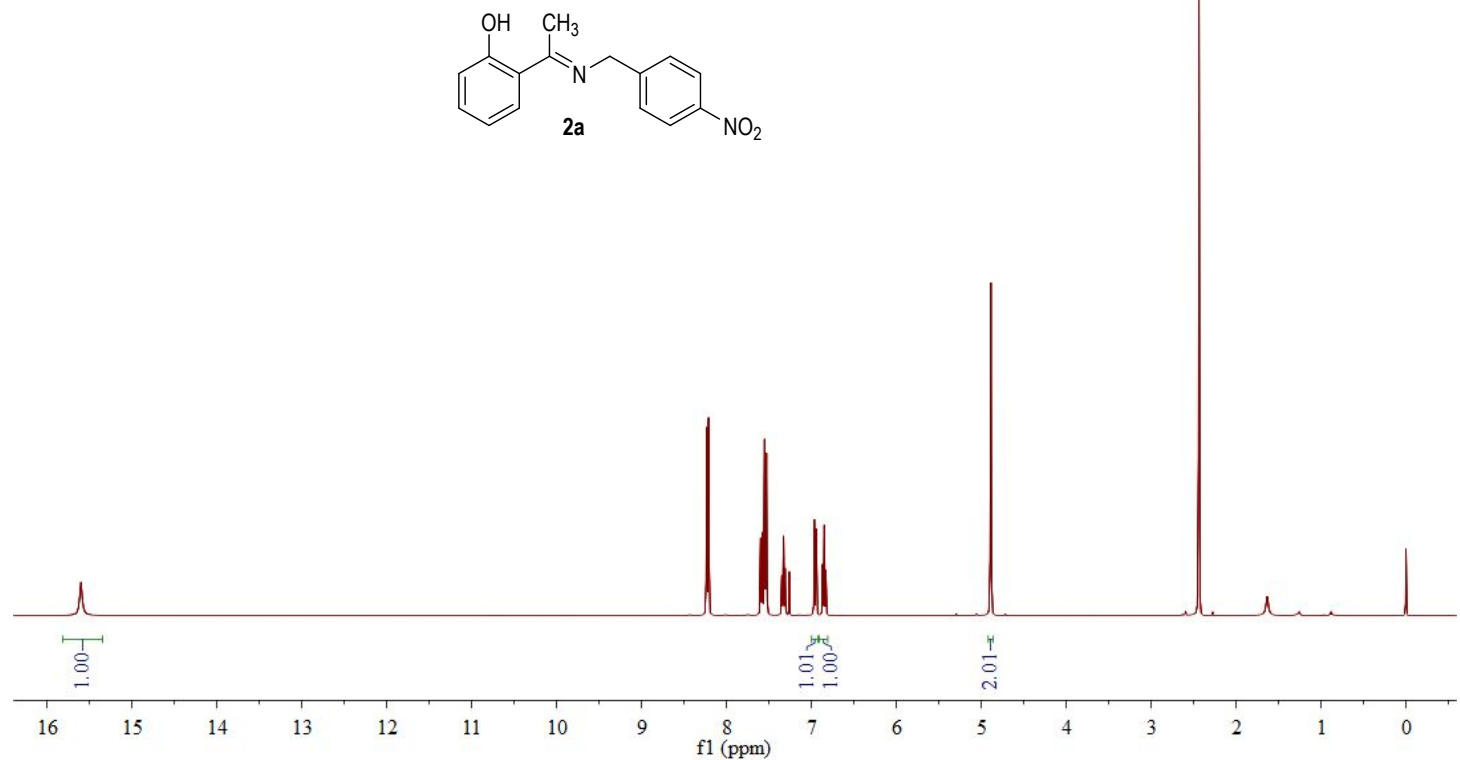

${ }^{1} \mathrm{H}$ NMR of compound $\mathbf{2 a}$

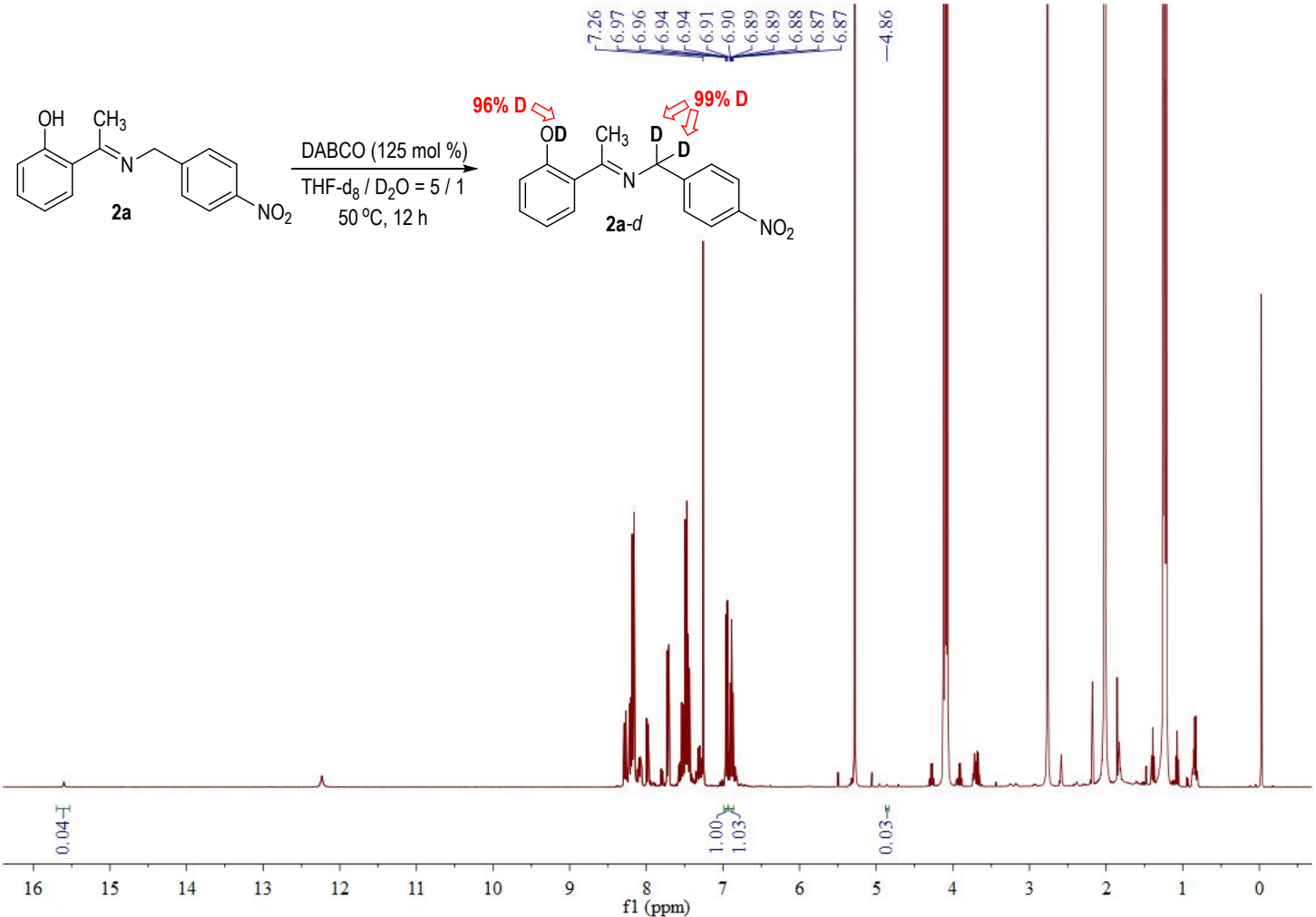

${ }^{1} \mathrm{H}$ NMR of the reaction mixture 
${ }^{1} \mathrm{H}$ NMR of compound 2aa in $\mathrm{CDCl}_{3}$

$$
\begin{aligned}
& 8 \\
& \substack{1 \\
\text { i }}
\end{aligned}
$$

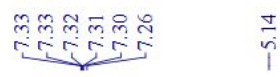

$\frac{1}{i}$<smiles>CC(=NCc1ccccc1[N+](=O)[O-])c1ccccc1O</smiles>
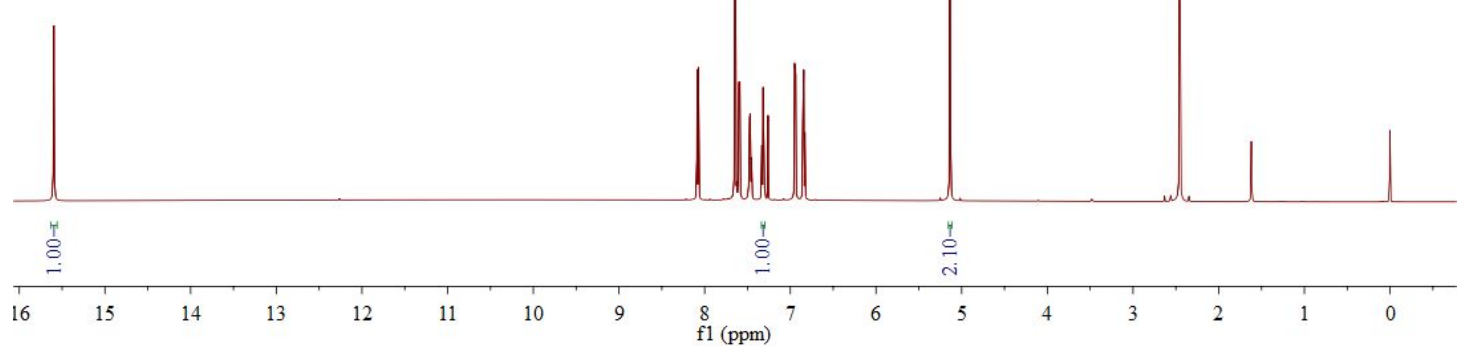

${ }^{1} \mathrm{H}$ NMR of compound $\mathbf{2 a a}$

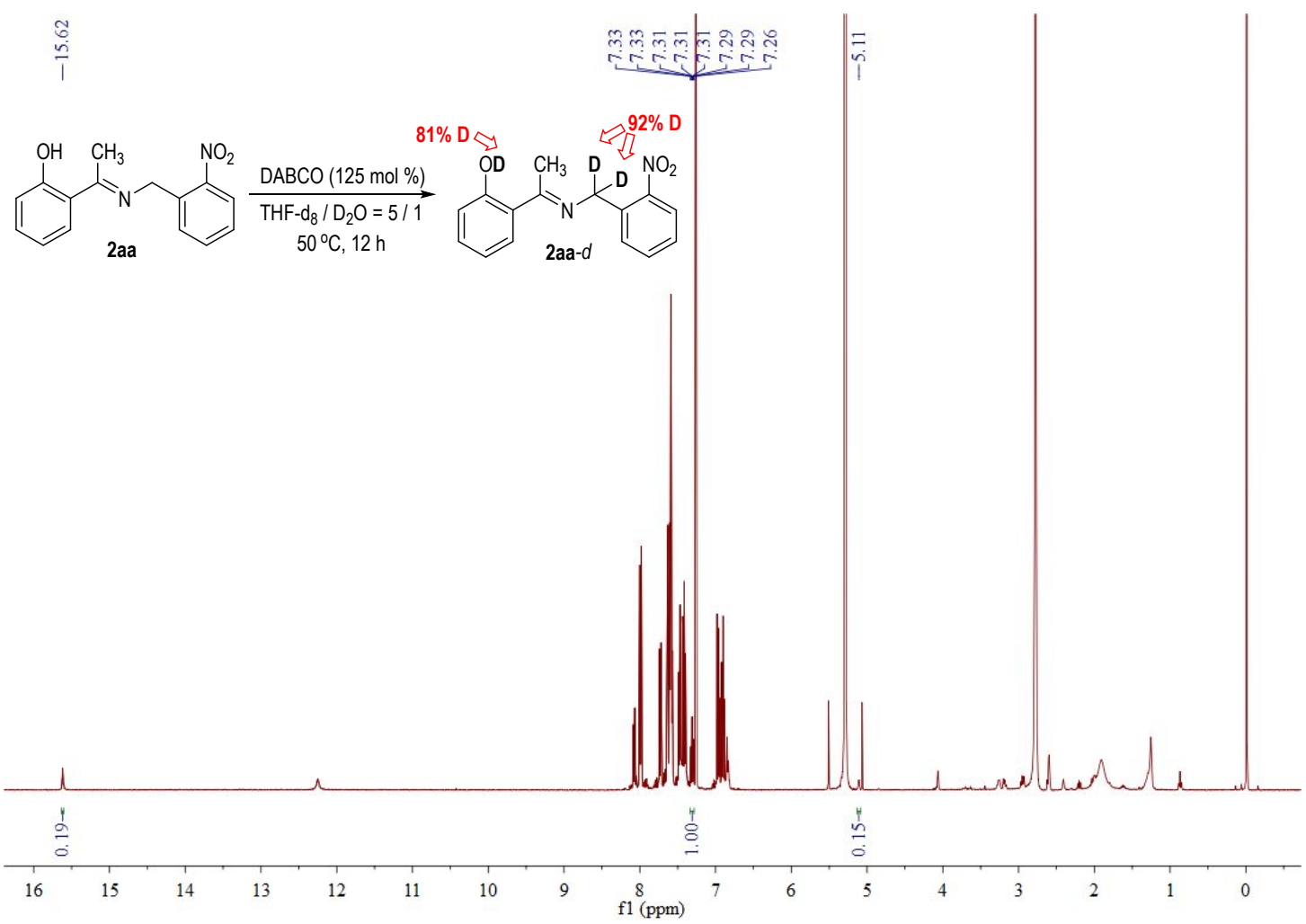

${ }^{1} \mathrm{H}$ NMR of the reaction mixture 

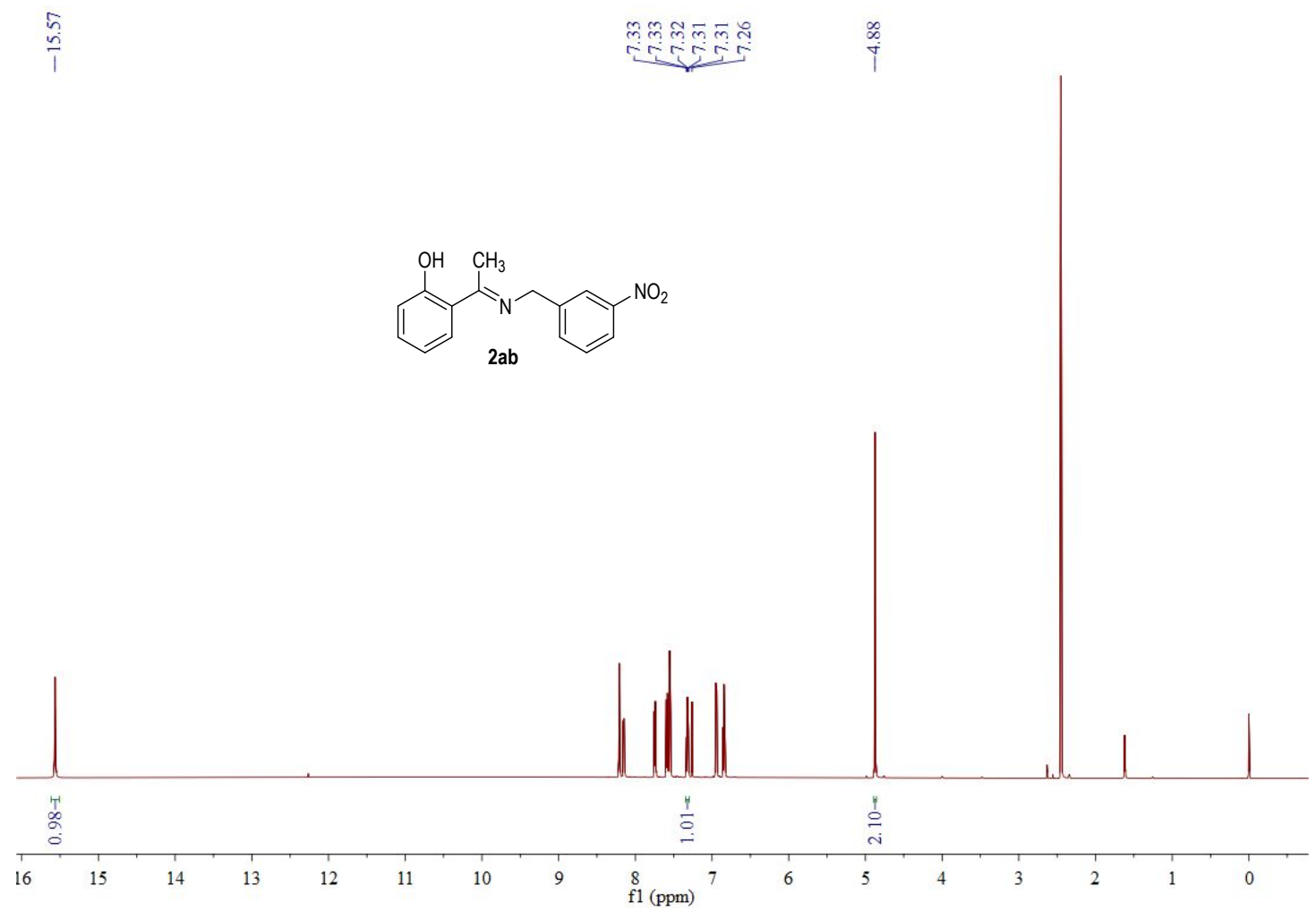

${ }^{1} \mathrm{H}$ NMR of compound $\mathbf{2} \mathbf{a b}$

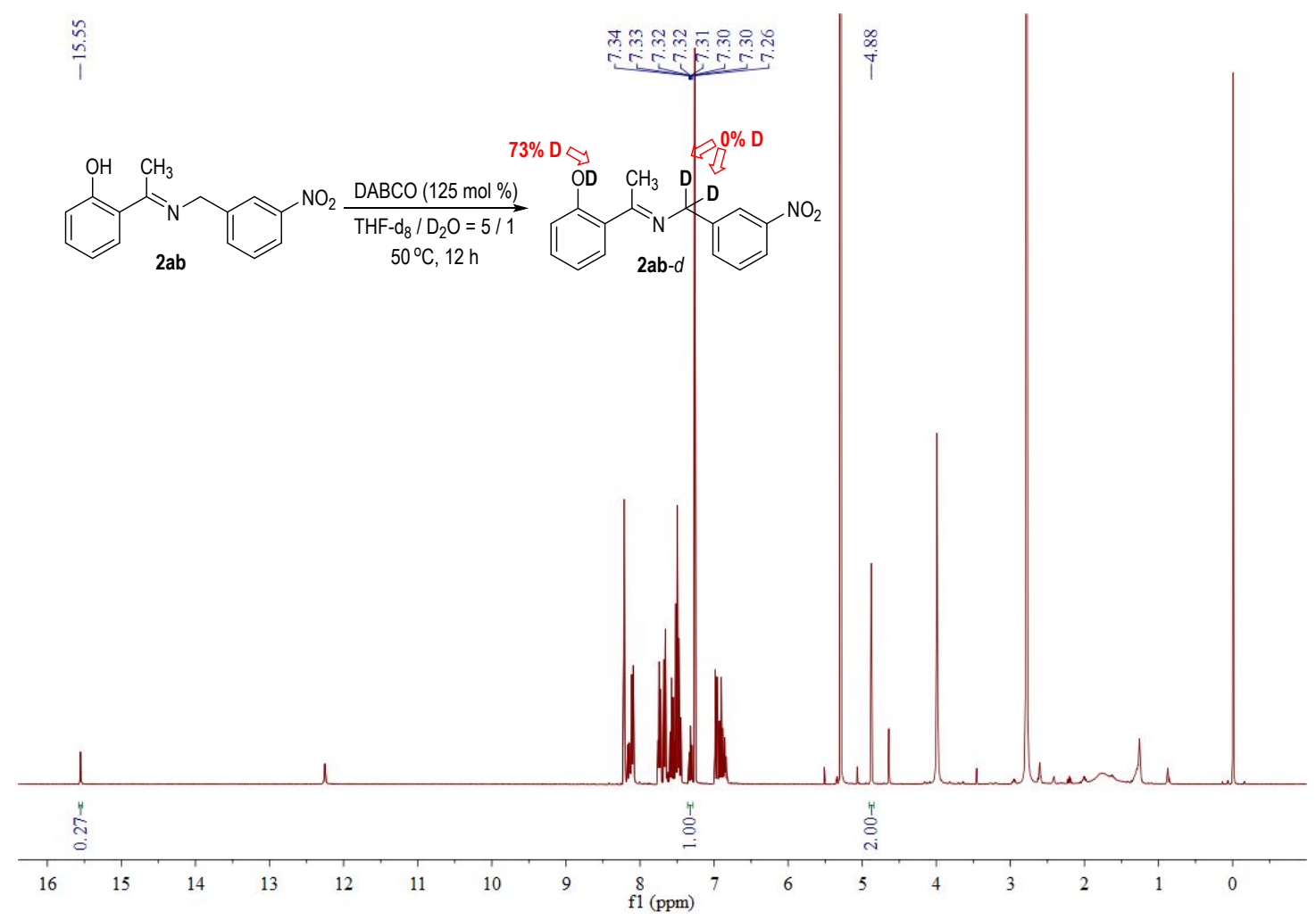

${ }^{1} \mathrm{H}$ NMR of the reaction mixture 
<smiles>C/C(=N\Cc1cc(C(F)(F)F)cc(C(F)(F)F)c1)c1ccccc1O</smiles>

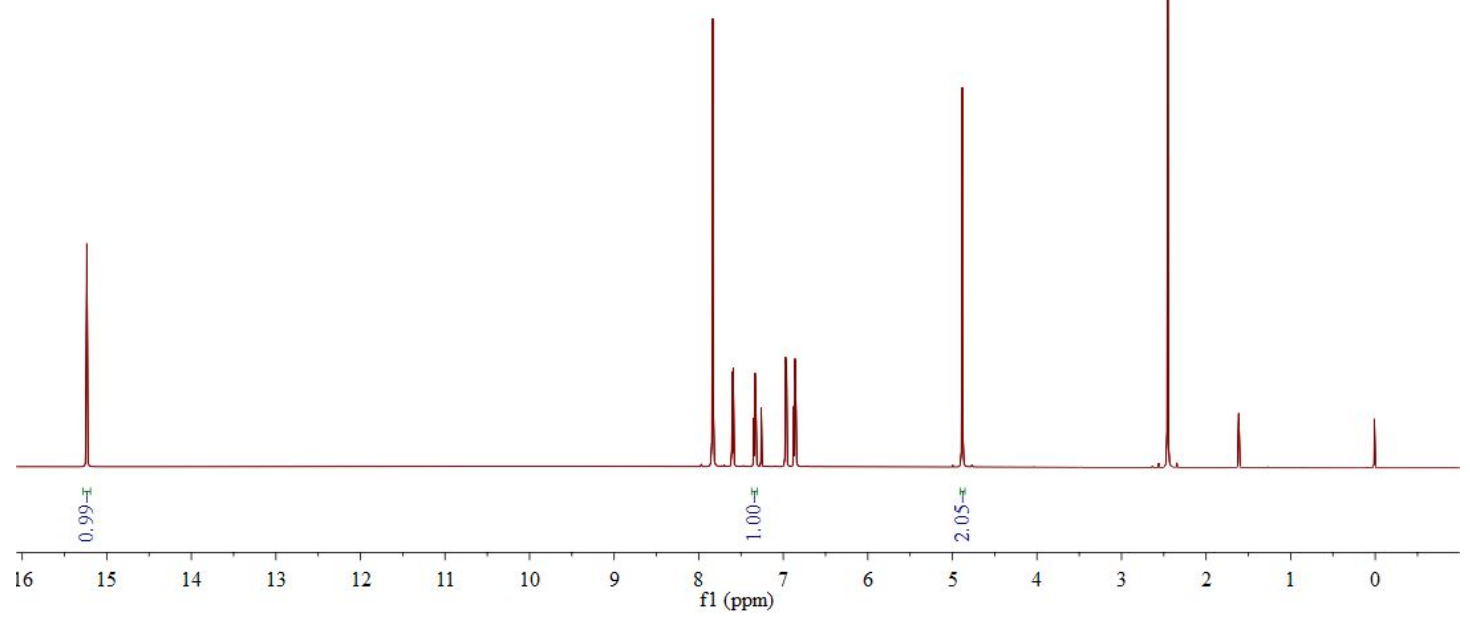

${ }^{1} \mathrm{H}$ NMR of compound $\mathbf{2 a c}$

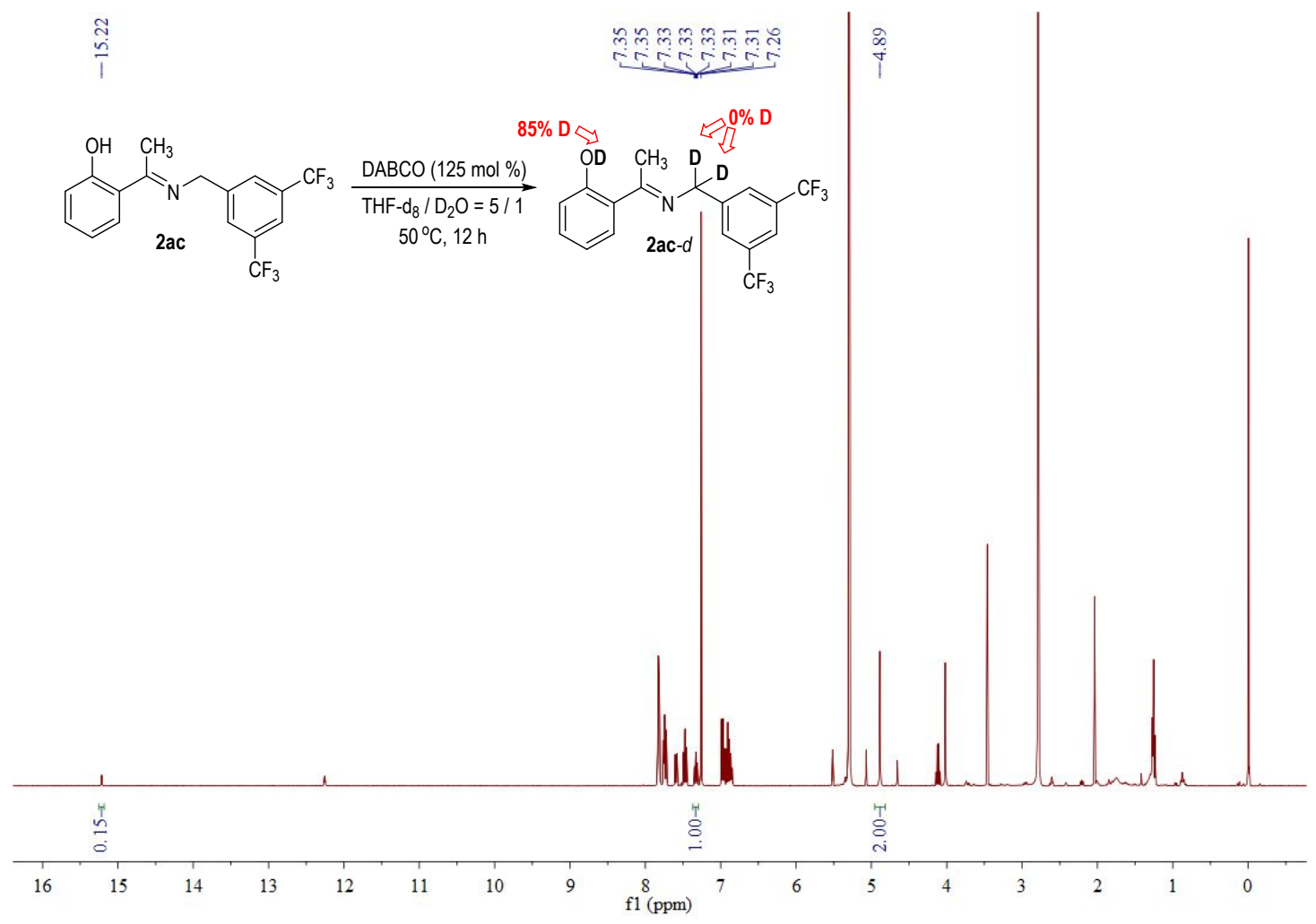

${ }^{1} \mathrm{H}$ NMR of the reaction mixture 


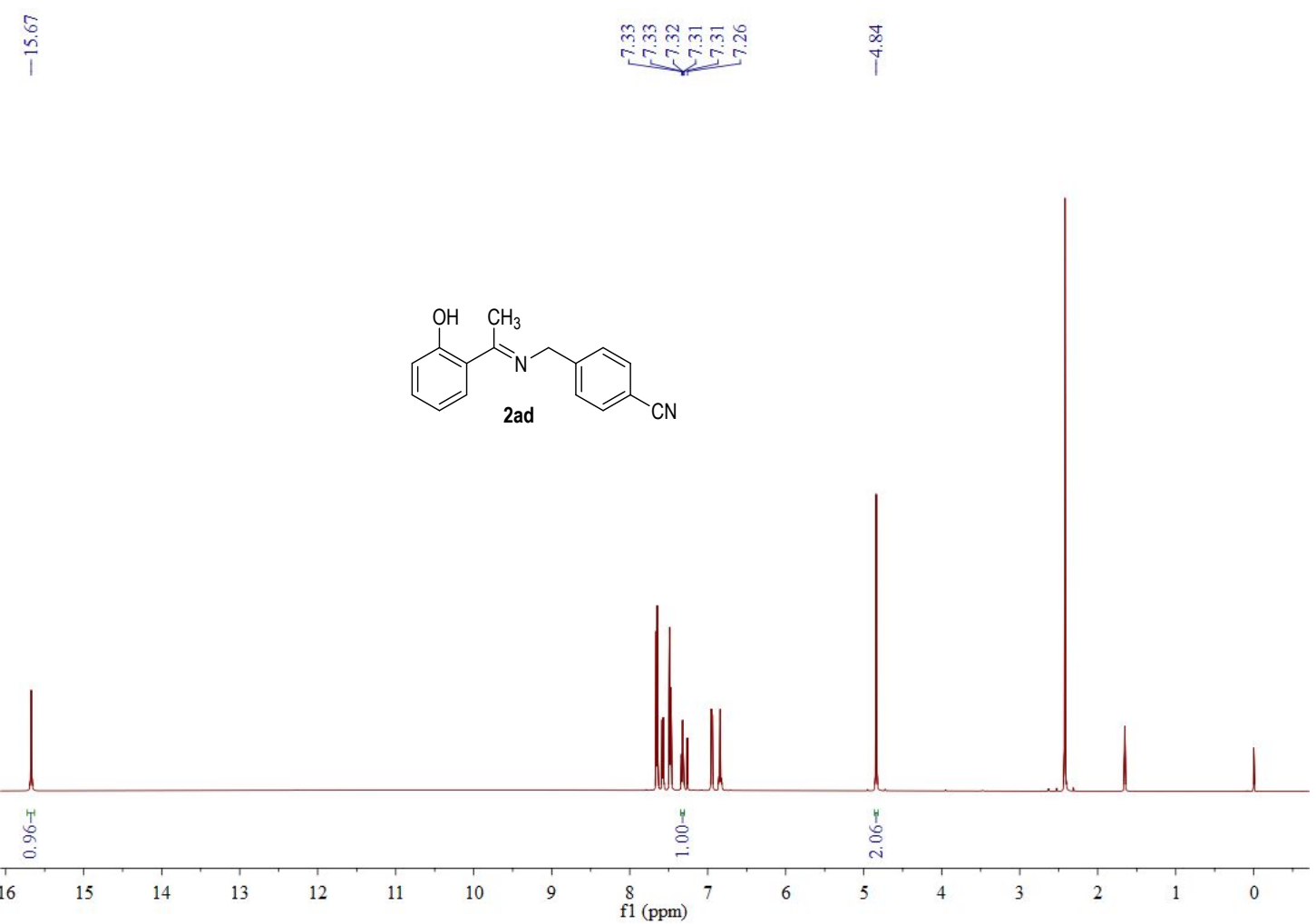

${ }^{1} \mathrm{H}$ NMR of compound $\mathbf{2 a d}$

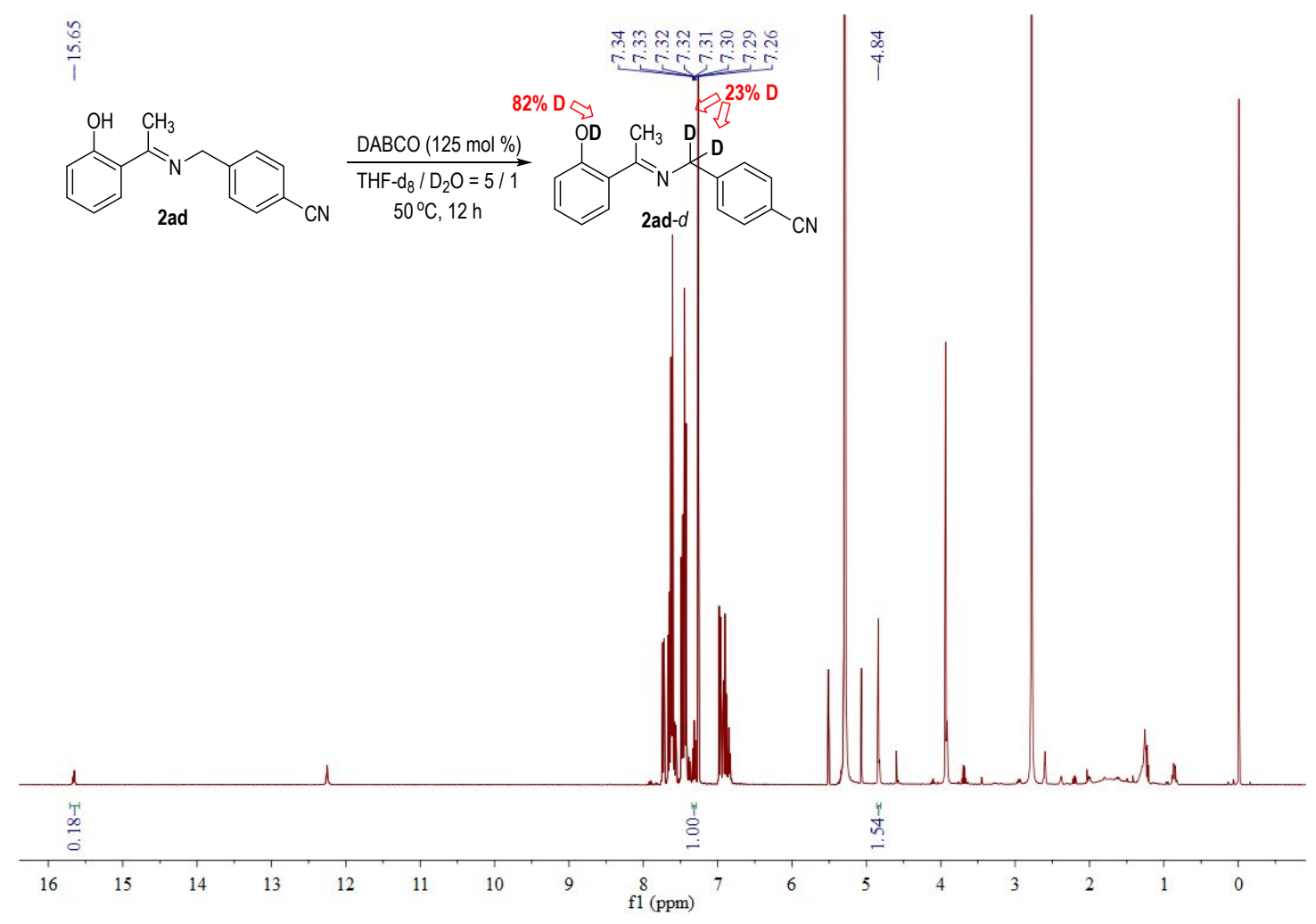

${ }^{1} \mathrm{H}$ NMR of the reaction mixture 


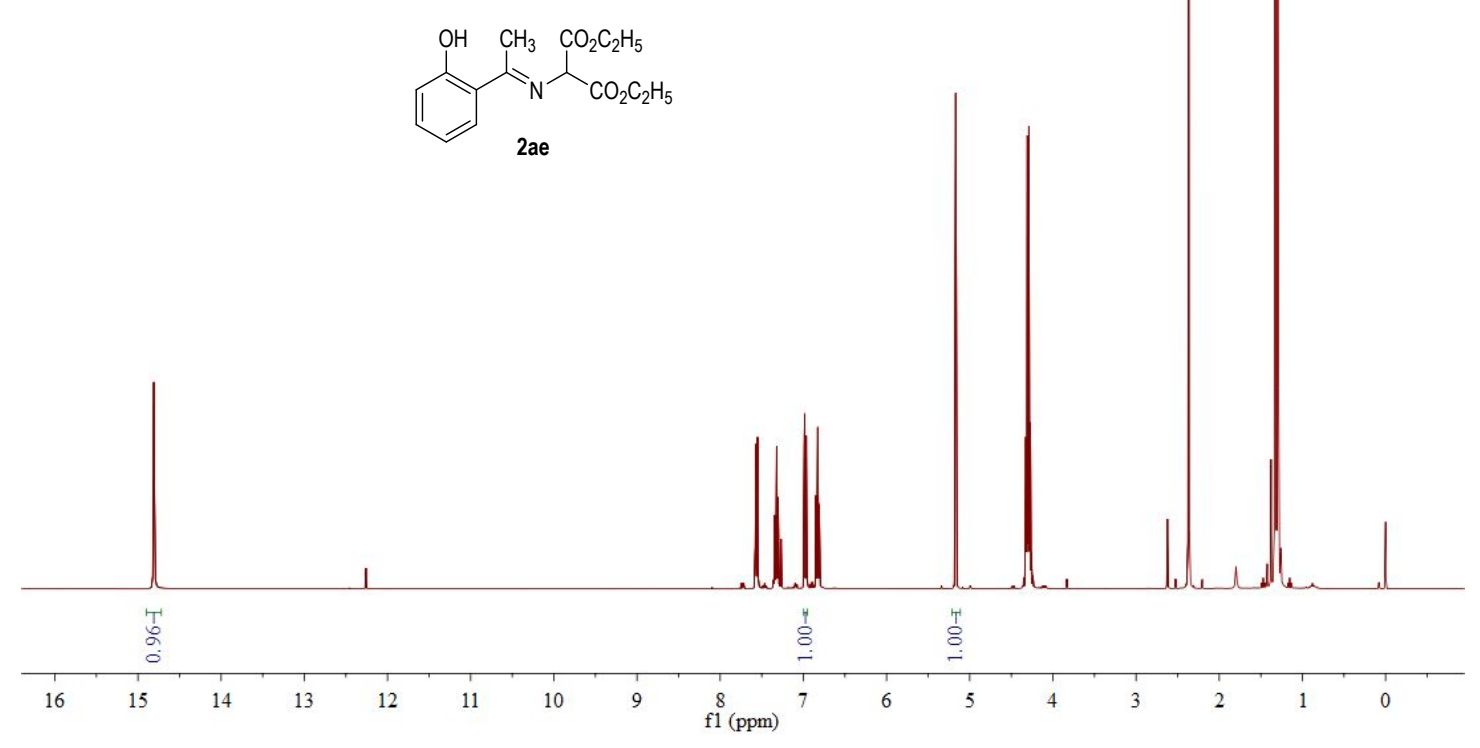

${ }^{1} \mathrm{H}$ NMR of compound 2 ae

$\underset{\frac{1}{8}}{\frac{2}{8}}$

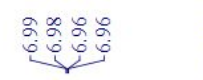

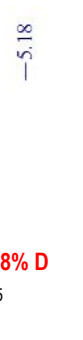

等

$71 \% \mathrm{D} \leadsto$

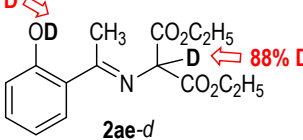

lid

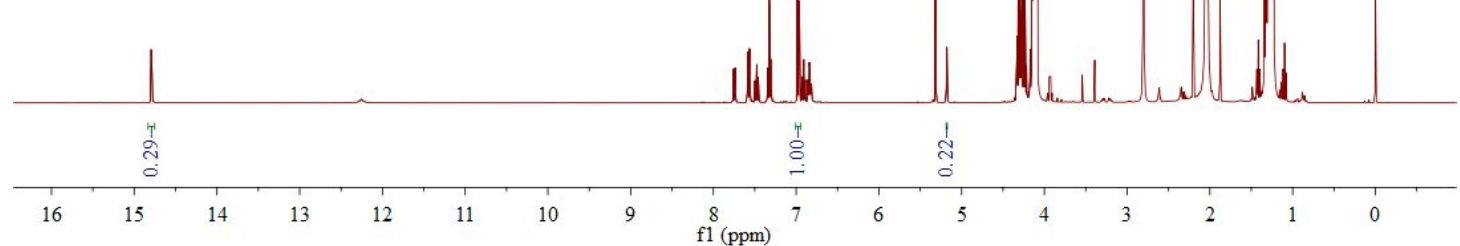

${ }^{1} \mathrm{H}$ NMR of the reaction mixture 